\title{
Characterizing the Neurobiological Consequences of Perinatal Exposure to the Obesogenic Endocrine Disruptor Bisphenol-A
}

\author{
Harry MacKay
}

A thesis submitted to the Faculty of Graduate \& Postdoctoral Affairs in partial fulfillment of the requirements of the degree of

Doctor of Philosophy

in

Neuroscience

Carleton University

Ottawa, ON

(C)2015 - Harry MacKay 


\section{Abstract}

Bisphenol-A (BPA) is a component of polycarbonate and many other types of plastics. BPA has been characterized as an endocrine disruptor, most famously due to observations of its estrogenic activity in various experimental models. Because BPA is a common constituent of food and drink containers, and because it can leach out under certain conditions, human exposure is nearly ubiquitous. In this thesis, we demonstrate that CD-1 mice exposed to low, environmentally relevant doses of BPA during the perinatal period exhibit an adult phenotype characterized by sex-specific metabolic disruptions. Specifically, male mice exhibit impaired glucose tolerance, and females a propensity toward diet-induced obesity. Given the sensitivity of the hypothalamus to the organizational effects of sex steroids, we explored the possibility that early-life BPA exposure adversely affects the development of hypothalamic feeding circuitry to bring about these effects. We found that BPA- and diethylstilbestrol (DES)-exposed pups have respectively delayed and blunted postnatal leptin surges - a state of affairs that points to a role for leptin in the organizational effects of early-life xenoestrogen exposure. qRT-PCR analysis of leptin mRNA expression in white adipose tissue collected at the same time suggests that BPA and DES act at the transcriptional level to bring about these effects. Both male and female BPA-exposed mice showed a reduced density of POMC projections into the PVN. This phenotype was rescued in female BPA-exposed animals given daily injections of supplemental leptin $(5 \mu \mathrm{g} / \mathrm{g} / \mathrm{day})$. Adult offspring from this experiment were resistant to leptin-induced suppression of food intake, body weight loss, and hypothalamic POMC upregulation. Taken together, these data suggest that BPA, a known obesogen, may exert its effects through developmental programming of the hypothalamic melanocortin circuitry. 


\section{Acknowledgements}

It is a pleasure to express my sincere gratitude to my supervisor Dr. Alfonso Abizaid for his years of patient guidance, reassurance, and most importantly his faith in my abilities as a researcher. With his diverse range of interests, collaborative spirit, and infectious curiosity, it really felt as though no topic was beyond the reach of our lab. I am also grateful and indebted to Drs. Barbara Woodside and John Stead, whose wisdom and guidance not only as members of my committee, but also in the broader scope of my career gave me much to look up to.

I feel very fortunate to have found my way to the Abizaid lab at Carleton's Department of Neuroscience not only because of its role in shaping my character as a researcher, but also because of the unforgettable environment of friends and collaborators who I have known over the years. None stand out more than Dr. Zack Patterson, with whom I've shared countless stimulating discussions, laughs, adventures and, for a brief period, even an office. His work ethic and attention to detail gave me a new standard to aspire to in my own career. My gratitude is also due to my friends Dr. Véronique St-Onge, Martin Wellman, Rim Khazall, Samantha King, Alex Edwards, and everybody else in the Abizaid lab for their help, friendship, and for the countless coffee runs.

I am deeply grateful to my parents for their love and support. Thanks to their encouragement, I always felt that I was doing something worthwhile, and making them proud. Finally I would like to thank Lauren, who offered nothing but patience, love, and encouragement to me, even though this all too often meant playing second fiddle to a room full of small rodents. Nobody has done more to broaden my horizons and give me the confidence to push forward in life.

H.M.

Ottawa, ON 


\section{List of Abbreviations}

$\alpha$-MSH: $\alpha$-Melanocyte Stimulating Hormone, 11 $\beta$-HSD: $11 \beta$-hydroxysteroid dehydrogenase, 5-HT: 5-hydroxytryptamine (serotonin), AgRP: Agouti-related Peptide, ANOVA: Analysis of Variance, AR: Androgen receptor, ARC: Arcuate nucleus, ARE: Androgen response element, AUC: Area Under the Curve, AVPV: Anteroventral periventricular nucleus, BAT: Brown Adipose Tissue, BPA: Bisphenol-A, BrDU: 5-bromo-2'-deoxyuridine, C/EBP $\alpha$ : CCAAT/enhancer binding protein $\alpha$, cAMP: Cyclic adenosine monophosphate, DES: Diethylstilbestrol, DHT: Dihydrotestosterone, DMH: Dorsomedial hypothalamus, Dnmt1: DNA methyltransferase, EDC: Endocrine Disrupting Compound, ELISA: Enzyme-Linked Immunosorbent Assay, EPA: United States Environmental Protection Agency, ER $\alpha$ : Estrogen receptor $\alpha, \mathbf{E R} \beta$ : Estrogen receptor $\beta$, ERE: Estrogen response element, ERK: Extracellular signal regulated kinase, $\mathbf{E R R} \alpha$ : Estrogen-related receptor $\alpha, \mathbf{E R R} \beta$ : Estrogen-related receptor $\beta, \mathbf{E R R} \gamma$ : Estrogen-related receptor $\gamma$, FAS: Fatty acid synthase, G6pc: Glucose 6-phosphatase, GABA: $\gamma$-aminobutyric acid, GAD-67: Glutamic Acid Decarboxylase 67, GPDH: Glycerol-3-phosphate dehydrogenase, GPR30: G protein-coupled receptor 30, GR: Glucocorticoid receptor, GRE: Glucocorticoid response element, GTT: Glucose Tolerance Test, HFD: High-fat diet, JAK: Janus Kinase, LBD: Ligand-binding domain, LH: Lateral hypothalamus, LH: Luteinizing hormone, LOAEL: Lowest Observed Adverse Effect Level, LPL: Lipoprotein Lipase, MAPK: Mitogen-activated protein kinase, MC4R: Melanocortin-4 Receptor, MCH: Melanin Concentrating Hormone, MeCP2: Methyl-CpG-binding Protein 2, MMTV: Mouse mammary tumour virus, mPOA: Medial preoptic area, Mrp1: multidrug resistance-associated protein 1, NEFA: Non-esterified fatty acid, NPT: United States National Toxicology Program, NPY: Neuropeptide Y, Oatp4a1: Organic anion-transporting polypeptide 4a1, ObRb: Leptin receptor (long isoform), pERK: Phosphorylated extracellular signal-related kinase, PI3K: Phosphatidylinositol 3 kinases, POA: Preoptic area, POMC: Proopiomelanocortin, PPAR $\gamma$ : Peroxisome Proliferator Activated Receptor $\gamma$, PPRE: PPAR response element, PSA: ProstateSpecific Antigen, PVN: Paraventricular nucleus, qRT-PCR: Quantitative Real-Time Polymerase Chain Reaction, RER: Respiratory Exchange Ratio, RXR: Retinoid X Receptor, SDNPOA: Sexually dimorphic nucleus of the preoptic area, SERM: Selective Estrogen Receptor Modulator, Sf1: Steroidogenic factor 1, SOCS3: Suppressor of Cytokine Signalling 3, Srebf1: Sterol regulatory element-binding transcription factor 1, STAT3: Signal Transducer and Activator of Transcription 3, TH: Tyrosine hydroxylase, TZD: Thiazolidinedione, Ugt2b1: UDPglucuronosyltransferase type 2b1, vGlut1: Vesicular Glutamate Transporter 1, VMH: Ventromedial hypothalamus 


\section{Contents}

1 Bisphenol-A 1

1.1 Human Exposure to BPA . . . . . . . . . . . . . . . . . 1

1.2 Metabolism and Pharmacokinetics of BPA . . . . . . . . . 3

1.3 Pharmacological Targets of BPA . . . . . . . . . . . . . 6

1.3.1 Estrogen Receptors . . . . . . . . . . . . . . . 6

1.3.2 Androgen Receptor . . . . . . . . . . . . . . . . . 10

1.3.3 Peroxisome Proliferator-Activated Receptor $\gamma \ldots \ldots$. . . . . 11

1.3.4 Glucocorticoid Receptor . . . . . . . . . . . . . . 14

1.4 Effects of BPA on Sexually Dimorphic Hypothalamic Nuclei . . . . 15

1.5 Obesogenic Effects of BPA . . . . . . . . . . . . . . 17

1.6 Challenges in Endocrine Disruptor Research . . . . . . . . . . . 20

1.6.1 Modeling Oral Exposure . . . . . . . . . . . . . 20

1.6.2 Diethylstilbestrol, an Estrogenic Positive Control . . . . . . 21

1.6.3 Avoiding Litter Effects . . . . . . . . . . . . . . 22

2 The Neurobiology of Obesity 24

2.1 Hypothalamic Regulation of Energy Balance . . . . . . . . . . . . . . . 24

2.2 The Hypothalamic Melanocortin System . . . . . . . . . . . . . . 25

2.3 Developmental Models of Obesity . . . . . . . . . . . . . . . . 26

2.4 Estrogenic Regulation of Feeding and Metabolism . . . . . . . . . 28

2.4.1 Activational Effects of Estradiol on Feeding . . . . . . . . 28

2.4.2 Organizational Effects of Sex Steroids on Energy Balance . . 32

2.5 Epigenetic Imprinting $\ldots \ldots \ldots$

2.5.1 Epigenetics in Developmental Models of Obesity . . . . . . 34

2.5.2 Epigenetics in Sexual Differentiation and Endocrine Disruption 36

2.6 Summary of Remaining Questions . . . . . . . . . . . . . . . . 37 
3 Organizational Effects of Perinatal Exposure to Bisphenol-A and Diethylstilbestrol on Arcuate Nucleus Circuitry Controlling Food Intake and Energy Expenditure $\quad 38$

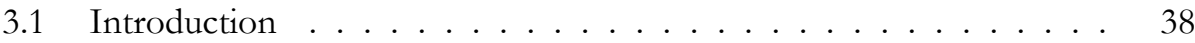

3.2 Methods . . . . . . . . . . . . . . . . . . . . . . 39

3.2 .1 Animals . . . . . . . . . . . . . . . . . 39

3.2.2 Weaning \& Early life . . . . . . . . . . . . . . . . . . . . . . 40

3.2.3 Estrus Cycle Monitoring . . . . . . . . . . . . . 40

3.2 .4 Diet-Induced Obesity . . . . . . . . . . . . . . . . . . . . . 41

3.2 .5 Indirect Calorimetry . . . . . . . . . . . . . . . . . 41

3.2.6 Glucose Tolerance Tests . . . . . . . . . . . . . . . . . . . 41

3.2 .7 Hormonal Analyses . . . . . . . . . . . . . . . . . . . . . . 42

3.2 .8 Body Fat Determination . . . . . . . . . . . . . . . 42

3.2 .9 Liver Histology . . . . . . . . . . . . . . . . . . . 42

3.2.10 Tissue Preparation for Histology . . . . . . . . . . . . . . 43

3.2.11 Double Immunohistochemistry for ER $\alpha$ and POMC . . . 43

3.2.12 Immunofluorescence for Vesicular Glutamate Transporter 1 (vGlut) or Glutamic Acid Decarboxylase 67 (GAD-67) and POMC, and AgRP . . . . . . . . . . . . . . . . . . 43

3.2 .13 Stereology . . . . . . . . . . . . . . . . 44

3.2.14 Quantification of vGlut1 and GAD67 Putative Synaptic Contacts ....................... 44

3.2.15 Quantification of POMC and AgRP Projections . . . . . . . 44

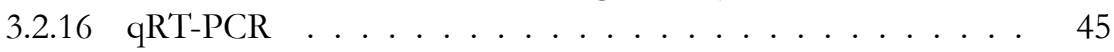

3.2 .17 Statistics ....................... 45

3.3 Results .......................... 46

3.3.1 BPA Exposure does not Affect Maternal Body Weight or Food

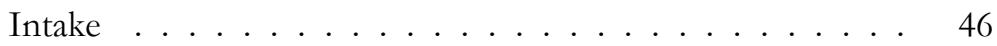

3.3.2 BPA-Exposed Female Offspring Become Hyperphagic and Obese When Fed a High-Fat Diet in Adulthood . . . . . . . 46

3.3.3 BPA Exposure Affects Energy Expenditure in Males . . . . 47

3.3.4 Males Exposed to BPA Have Impaired Glucose Tolerance . 48

3.3.5 Hepatic Lipid and Glycogen Content is Altered by Diet-Induced Obesity . . . . . . . . . . . . . . . . . . . 4 49

3.3.6 BPA-Exposed Females Develop Hyperleptinemia on a HighFat Diet . . . . . . . . . . . . . . . . . . . . . 49

3.3.7 BPA Exposure Affects Circulating Resistin and Insulin Levels on a High-Fat Diet . . . . . . . . . . . . . . . . . . . 49

3.3.8 Reduced POMC Projections to the PVN in BPA-Exposed Animals . . . . . . . . . . . . . . 50 
3.3.9 The Balance of Excitatory and Inhibitory Synapses onto POMC Neurons is Sexually Dimorphic, but not Affected by BPA or DES Treatment . . . . . . . . . . . . . 50

3.3.10 The Proportion of POMC Neurons that Co-Express ER $\alpha$ is Sexually Dimorphic, and Increased by BPA Exposure . . . . 50

3.3.11 BPA Exposure Affects the Transcriptional Response to DietInduced Obesity . . . . . . . . . . . . . . 51

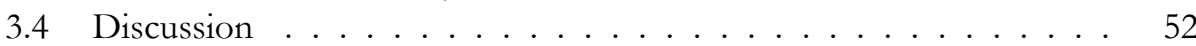

4 Perinatal Exposure to Low-Dose Bisphenol-A and Impaired Central and Behavioural Leptin Sensitivity in Adulthood $\quad 71$

4.1 Introduction . . . . . . . . . . . . . . . . . 71

4.2 Methods . . . . . . . . . . . . . . . . . 72

4.2 .1 Animals ..................... 72

4.2.2 Leptin Challenge at P16 . . . . . . . . . . . . . . . . . . 73

4.2 .3 pSTAT3 Immunofluorescence . . . . . . . . . . . . . . 73

4.2.4 Quantification of pSTAT3 Immunopositive Nuclei . . . . 74

4.2 .5 Estrous Cycle Monitoring . . . . . . . . . . . . . . . . . . 74

4.2.6 Fast/Refeed Challenge . . . . . . . . . . . . . . 75

4.2 .7 Leptin Challenge . . . . . . . . . . . . . . . . . . 75

4.2.8 Sacrifice and Tissue Preparation . . . . . . . . . . . . 75

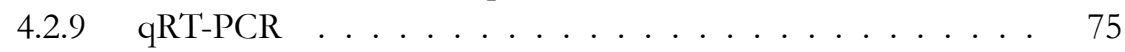

4.3 Results ......................... 75

4.3.1 Effects of BPA and DES Treatment on Hypothalamic LeptinInduced $\mathrm{pSTAT3}$ at P16 . . . . . . . . . . . . 75

4.3.2 Estrous Cyclicity is Unaffected by BPA or DES Treatment . 76

4.3.3 Rebound Feeding is Greater in Females, but not Affected by BPA or DES Treatment . . . . . . . . . . . . . . 77

4.3.4 Attenuated Leptin Sensitivity in BPA- and DES-Exposed Animals . . . . . . . . . . . . . . 77

4.4 Discussion . . . . . . . . . . . . . . . . . . . 78

5 Perinatal Exposure to Low-Dose Bisphenol-A (BPA) Alters the Postnatal Leptin surge Leading to Impaired Development of Hypothalamic $\begin{array}{lr}\text { Feeding Circuits } & \mathbf{8 8}\end{array}$

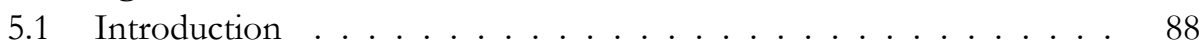

5.2 Methods . . . . . . . . . . . . . . . . . . . . 89

5.2 .1 Animals . . . . . . . . . . . . . . . . . . . 89

5.2 .2 BrDU Injection . . . . . . . . . . . . . . . . . . 91

5.2.3 Postnatal Leptin Supplementation . . . . . . . . . . . . . 91 
5.2.4 Serum and Tissue Collection . . . . . . . . . . . . . 92

5.2 .5 Body Fat Determination . . . . . . . . . . . . . . . . 92

5.2.6 Measurement of Circulating Leptin . . . . . . . . . . . . . . 92

5.2.7 qRT-PCR Measurement of Leptin Expression . . . . . . . . 92

5.2.8 Immunofluorescence for POMC and BrDU . . . . . . . 93

5.2.9 Immunofluorescence for POMC and AgRP . . . . . . . . . . 93

5.2.10 Quantification of BrDU Immunopositive Nuclei . . . . . . . 93

5.2.11 Quantification of POMC \& BrDU Co-Localization . . . . . 94

5.2.12 Quantification of POMC and AgRP Projections . . . . . . . . 94

5.2 .13 Statistics . . . . . . . . . . . . . . . . . . 94

5.3 Results .............................. 95

5.3.1 Maternal and Pup Characteristics are not Affected by BPA or DES Treatment . . . . . . . . . . . . . . . 95

5.3.2 Neurogenesis at E12 is not Affected by BPA or DES Treatment 95

5.3.3 Embryonic Neurogenesis of POMC Neurons is not Affected by BPA or DES Treatment . . . . . . . . . . . . . 95

5.3.4 Hypothalamic POMC Projections are Reduced in Males and Females Exposed to BPA . . . . . . . . . . . . . . 95

5.3.5 BPA and DES Disrupt the Postnatal Leptin Surge . . . . . . 96

5.3.6 Body Weight and Composition is not Affected by Postnatal Leptin Treatment . . . . . . . . . . . . . . . 96

5.3.7 Postnatal Leptin Treatment Rescues Impaired POMC Projections in Females Exposed to BPA . . . . . . . . . . . . . . . 97

5.4 Discussion . . . . . . . . . . . . . . . . . . . 97

6 General Discussion $\quad 112$

6.1 Is BPA Acting as a Xenoestrogen? . . . . . . . . . . . . . . . . . . 112

6.1.1 Inferences From Sex Differences . . . . . . . . . . . . 112

6.1.2 Inferences From the Effects of DES . . . . . . . . . . . . 114

6.2 Is BPA Hazardous to Humans? . . . . . . . . . . . . . . . . . . . 118

6.2.1 Developmental Programming in Humans . . . . . . . . . 120

6.2.2 Perinatal Leptin in Humans . . . . . . . . . . . . . . . . . . . . . . . . . . . . . . . . . . . . . . . .

6.3 Conclusion . . . . . . . . . . . . . . . . . . . 122 
A Details on Experimental Methods $\quad 124$

A.1 Detailed Diet Composition . . . . . . . . . . . . . . . . . . . 124

A.2 Primers for qRT-PCR . . . . . . . . . . . 125

B Copyright Documentation $\quad 126$

B.1 Permission from Endocrinology . . . . . . . . . . . . . 126

B.2 Co-Author Permission Statements . . . . . . . . . . . . . . . . 127 


\section{List of Figures}

3.2 Adult body weight and caloric intake . . . . . . . . . . . . 58

3.3 Adult fat pad mass . . . . . . . . . . . . . . . . . . . . . 59

3.4 Metabolic parameters at two months . . . . . . . . . . . 60

3.5 Metabolic parameters at five months . . . . . . . . . . . . . 61

3.6 Glucose tolerance test . . . . . . . . . . . . . . . . . 62

3.7 Hepatic Oil Red $\mathrm{O}$ and periodic acid-Schiff staining . . . . . . . . 63

3.8 Adult circulating leptin . . . . . . . . . . . . . . . . 65

3.9 Immunofluorescence analysis of hypothalamic structure . . . . . . 66

$3.10 \mathrm{ER} \alpha$ and POMC immunohistochemistry . . . . . . . . . . 68

3.11 Gene expression in the arcuate nucleus . . . . . . . . . . . 69

4.2 Leptin-induced pSTAT3 immunofluorescence at P16 . . . . . . . . . . . 82

4.3 Estrous cycle . . . . . . . . . . . . . . . . . . . . . . 84

4.4 Food intake over the estrous cycle . . . . . . . . . . . . . . 85

4.5 Overnight fast/refeed challenge . . . . . . . . . . . . . 85

4.6 Baseline body weight and food intake . . . . . . . . . . . 86

4.7 Metabolic response to leptin . . . . . . . . . . . . . . . . 86

4.8 Leptin-induced gene expression in mediobasal hypothalamus . . . . 87

5.2 Maternal and litter data . . . . . . . . . . . . . . . . . . . . . 101

5.3 BrDU immunofluorescence at P16 . . . . . . . . . . . . . 103

5.4 Colocalization of BrDU and POMC in the ARC at P21 . . . . . . . 106

5.5 POMC projections to the PVN at P21 . . . . . . . . . . . . . 107

5.6 Postnatal leptin surge . . . . . . . . . . . . . . . . . . . . . 108

5.7 Relative body weight of leptin-treated animals . . . . . . . . . . . . . 109

$5.8 \quad$ Fat pad mass at P21 . . . . . . . . . . . . . . . . . . 110

5.9 POMC and AgRP projections to the PVN in leptin-treated animals . 111 


\section{List of Tables}

3.1 Maternal body weight across pregnancy and lactation . . . . . . . . 57

3.2 Maternal food intake across pregnancy and lactation . . . . . . . . . . 57

3.3 Estimated maternal doses of BPA and DES . . . . . . . . . . . 57

3.4 Circulating IL-6, insulin, and resistin . . . . . . . . . . . . . . 64

5.1 Estimated maternal doses of BPA and DES . . . . . . . . . . . 102

6.1 Concordance between BPA and DES animals . . . . . . . . . . 115

A.1 Composition of diets used in these studies . . . . . . . . . . . . . . 124

A.2 Primer sequences for qRT-PCR . . . . . . . . . . . . 125 


\section{Chapter 1}

\section{Bisphenol-A}

Bisphenol-A (BPA, 2,2-bis (4'-hydroxyphenyl) propane) is a chemical plasticizer and monomeric constituent of polycarbonate plastics and epoxy resins. BPA was first synthesized in 1891 by the Russian chemist A.P. Dianin by the condensation of acetone with two equivalents of phenol with a strong acid as a catalyst. It is from the inclusion of acetone that BPA gets its name; other bisphenols derive from different reactants and thus differ in the last letter of their name. For example, Bisphenol-S is made by the reaction of phenol and sulfur trioxide, and Bisphenol-F is prepared with formaldehyde ${ }^{1}$. The relative ease of production, combined with the favourable chemical and physical properties of of BPA-containing plastics has driven demand for this chemical and the products of which it is a component. BPA is produced in quantities approaching three million metric tonnes annually [3], making it one of the highest volume chemicals currently in production. In the case of polycarbonate plastic, polymerized BPA is the primary constituent. In the case of plastics for which it is not the primary ingredient, BPA is often at least a key ingredient. Employed as a precursor of epoxy resins, BPA can be found in the resinous linings of canned foods and beverage containers. BPA is also found as an ingredient in dental sealants, and in trace quantities in certain printing inks and thermal paper [4]. Owing to its role as an ingredient in such a diverse range of products, the risk of exposure to BPA borders on unavoidable in the modern world.

\subsection{HUMAN EXPOSURE TO BPA}

The ubiquity of BPA in food and beverage containers raises important questions about its stability. Polymerized BPA is effectively sequestered only as long as that polymer remains intact. Unpolymerized, free BPA leaches readily into a variety of solvents and food products. All polycarbonate plastics contain a certain quantity of residual

\footnotetext{
${ }^{1}$ These and other bisphenols are sometimes offered as substitutes for BPA. Though less extensively tested in-vivo, there is every reason to expect these alternatives to have biological effects that are comparable to BPA $[1 ; 2]$.
} 
unpolymerized BPA with concentrations in the plastic ranging from 1-140 mg/ $\mathrm{kg}$ [4], depending on the efficiency of the polymerization reaction. While this issue could potentially be obviated by stricter quality control standards on polycarbonate products, a much more pressing concern is the potential for certain situations to actively promote depolymerization and allow BPA to leach into contained foodstuffs ${ }^{2}$. The relative concentration of free BPA can be increased by exposure to high temperatures, acidic or alkaline foodstuffs, and ethanol; any of which could be expected in the life of a food or drink container $[4 ; 6 ; 7]$. The conditions found inside dishwashing machinesharsh alkaline detergents and high heat in particular-may also increase the rate of BPA leaching, though the largest effects are obtained only after incomplete rinsing [8]. The rate of BPA leaching tends to increase over a container's lifespan such that older containers, having been exposed to a variety of foodstuffs and a number of wash cycles, are likely to leach BPA at a much higher rate than new containers [7]. Polycarbonate containers tend to retain their structural integrity as they age, and so containers may remain useful and apparently safe long after they have begun releasing high levels of BPA.

Because BPA does not accumulate in body tissue to any significant degree, it is possible to obtain an rough estimate of an individual's daily exposure to the compound by an assessment of urinary concentration. It is common to test single spot samples of urine to estimate exposure, but this approach must be used with caution, for BPA levels in urine and serum vary markedly across the day depending on the time of the last meal [9]. Still, a study possessing sufficient statistical power is unlikely to grossly mis-estimate the average daily exposure, provided that samples are collected at intervals throughout the day. Christensen et al. found little difference in median urinary BPA concentration between surveys using spot collection, 24-hour collection and 'first morning' samples, though sample variance was unsurprisingly much lower in 24-hour studies [10].

Exposure to BPA is widespread in North America, with between $91-93 \%$ of adults showing measurable levels in urine $[11 ; 12]$. Health Canada's Bureau of Chemical Safety estimated in its 2012 report that the average daily intake among Canadians over the age of nine is $0.055 \mu \mathrm{g} / \mathrm{kg} / \mathrm{day}$ [12]. Similar values were obtained in 2012 by LaKind et al., who compared exposure among both Americans and Canadians finding mean daily intakes of $0.043 \mu \mathrm{g} / \mathrm{kg} /$ day and $0.073 \mu \mathrm{g} / \mathrm{kg} /$ day respectively [11].

The sort of large cross-sectional studies discussed in the previous paragraph allow for a rough estimation of incidental exposure to BPA among a population. These studies carry a major tradeoff, however, in their inability to measure the moment-tomoment kinetic details of BPA exposure. Teeguarden et al., designed a clinical exposure

\footnotetext{
${ }^{2}$ At times this phenomenon has confounded the scientific process, as when Krishnan et al., revealed that a putative yeast-derived estrogenic product they had previously identified was actually BPA released from culture flasks during autoclaving [5].
} 
study to avoid this tradeoff. Participants consumed a controlled meal rich in canned foods and juices, mimicking a 'worst case' scenario for typical BPA exposure. Surprisingly, the authors found that the net 24-hour BPA exposure, as measured by urine BPA content, averaged $0.27 \mu \mathrm{g} / \mathrm{kg}$ which is higher than the previously estimated $95^{\text {th }}$ percentile of human exposure in the United States [9]. While BPA was detectable in most urine samples, the authors found limited success in measuring BPA concentrations in serum. Reliably detectable levels of BPA were only observed in the serum of those with the highest urinary concentrations [9]. This suggests that BPA dwells only briefly in the serum before being excreted, and this rapid excretion is likely to be a relevant factor for risk assessment. vom Saal et al., criticized this study on several points, the most serious of which was that actual BPA exposure was not measured nor controlled for, making it impossible to know whether the model was truly representative [13].

Though adult exposure to BPA is certainly a concern, prenatal and infantile exposure poses a much greater risk for public health. The glucuronidation pathways that normally serve to modify the BPA molecule and prepare it for excretion are not fully active in neonatal animals (see Section 1.2), and so a given dose of BPA would be expected to persist for a longer period of time, and have a greater range of effects when compared to an adult. Ethical concerns preclude experimental study in infants, but a great deal can be inferred by studying incidental BPA levels in neonatal samples taken for other reasons. In Canada, probable daily intake values may reach as high as $1.31 \mu \mathrm{g} / \mathrm{kg} /$ day in infants aged 2-3 months consuming large amounts of formula [14]. The European Food Safety Authority estimated a similarly high figure of $2.3 \mu \mathrm{g} / \mathrm{kg} / \mathrm{day}$ for 3 month old infants consuming primarily formula, and a still higher $11 \mu \mathrm{g} / \mathrm{kg} / \mathrm{day}$ for infants using a polycarbonate bottle [15]. Accordingly, polycarbonate baby bottles have been banned in Canada, the European Union, and the United States, among other jurisdictions.

The United States Environmental Protection Agency has established $50 \mu \mathrm{g} / \mathrm{kg} / \mathrm{day}$ as a reference dose for BPA [11]. The Food Directorate of Health Canada has similarly established a provisional tolerable daily intake of $25 \mu \mathrm{g} / \mathrm{kg} /$ day [14]. Being well above observed levels of human exposure, these figures are intended to set an upper limit on BPA exposure and, in a sense, demonstrate that the doses to which humans are typically exposed are unlikely to be harmful.

\subsection{METABOLISM AND PHARMACOKINETICS OF BPA}

Though human exposure to BPA through routes as diverse as the transdermal and respiratory is possible, the bulk of exposure for most people seems to come from the oral route of administration [16]. Oral administration of BPA subjects the compound to first-pass metabolism through the hepatic portal system. First-pass metabolism greatly decreases the proportion of bioavailable BPA that reaches systemic circulation. Stud- 
ies in rats using doses of $10 \mathrm{mg} / \mathrm{kg}$ and $100 \mathrm{mg} / \mathrm{kg}$ show that BPA is rapidly absorbed through the digestive tract, reaching detectable levels in plasma within 10 minutes of oral gavage [17]. Intraperitoneal or intravenous routes of administration result in relatively higher proportions of circulating bioavailable BPA [18]. Glucuronidation is a process common in the metabolism of hydrophobic molecules, the end result of which is to increase the water solubility of the substrate, allowing it to be excreted. BPA is readily glucuronidated in the liver by the Ugt $2 \mathrm{~b} 1$ isoform of the UDP-glucuronosyltransferase family of enzymes [19]. This process also seems to occur in the lining of the small intestine and colon as BPA is absorbed [20]. Glucuronidated BPA does not induce ER transactivation in MCF-7 and HEPG2 cell line-based assays, nor does it compete for binding to $\mathrm{ER} \alpha$ and $\mathrm{ER} \beta$ with estradiol, and for these reasons it is considered to be biologically inactive, at least with respect to its estrogenic potential [21; 22]. Studies in which rat livers were directly perfused with various concentrations of BPA solution attest to the speed and efficiency of hepatic glucuronidation, showing that only a small fraction of free BPA ends up passing into systemic venous circulation [23]. Glucuronidated BPA leaves the liver through the biliary system, and is deposited into the duodenum without any additional formalities [23]. Regardless of route of administration, virtually all administered BPA leaves the body within 48 hours with no significant amount of retention in bodily tissues $[18 ; 17]$. In rats, the majority $(\sim 80 \%$ in males, $\sim 65 \%$ in females) of BPA can be found in the feces, a consequence of BPA's biliary excretion. The next largest fraction is found in urine $(\sim 15 \%$ in males, $\sim 25 \%$ in females) [18]. By the time the intestinal contents reach the cecum, unconjugated BPA concentrations rebound substantially, falling off again in the colon [24]. This can be accounted for by the presence of cecal bacteria with high levels of $\beta$-glucuronidase which may deconjugate BPA and allow for its release back into circulation [24]. In this way, orally ingested BPA may recirculate through the body several times before finally being excreted, a process known as enterohepatic recirculation [17; 24]. Since humans neither possess ceca nor excrete BPA via the biliary system, it is likely that this marks an important difference in pharmacokinetics between the species.

Human studies using $\mathrm{d}_{16}$ - $\mathrm{BPA}$ at $5 \mathrm{mg} /$ person confirm that-as in rats- the major metabolite of BPA is BPA-glucuronide [25]. The glucuronidation process following oral exposure proceeds efficiently, and reaches its projected maximum plasma concentration within 1.3 hours of ingestion [25]. The resulting metabolite appears in urine shortly thereafter. In humans, urine is the primary means by which BPA-glucuronide is eliminated from the body, because the molecular weight of that complex is below the threshold for biliary excretion [25]. This marks an important contrast with rodents which, for reasons not well understood, have a different threshold for biliary excretion. Since BPA-glucuronide avoids the gastrointestinal tract, there is little risk of it encountering bacterial $\beta$-glucuronidase and thus little risk of enterohepatic recirculation. Such differences are an important consideration when interpreting findings from 
animal models of BPA exposure [26].

The greatest risk associated with BPA exposure is likely not its acute effects in fully-formed adult animals, but rather the organizational effects it may exert in its capacity as an endocrine disruptor in developing animals. It cannot be safely assumed that adult metabolic pathways are in place during all stages of development, and therefore the effects of BPA may be markedly more severe in young animals. Research into this has shown that hepatic Ugt2b1 mRNA expression and enzymatic activity is virtually non-existent in fetal and newborn rats [27], and only reaches adult levels by P21-approximately the time of weaning [28]. Moreover, pregnancy is associated with changes in BPA metabolism that may expose fetal and neonatal animals to higher levels than would be expected if adult non-pregnant female pharmacokinetics are taken as the standard. Nulliparous females have a higher rate of glucuronidation and excretion of BPA, as well as elevated hepatic expression of Ugt2b1 as compared to males [29; 30], but this relative advantage disappears during pregnancy. In pregnant rats, hepatic microsomal glucuronosyltransferase activity for BPA and diethylstilbestrol (DES) respectively declines to $\sim 60 \%$ and $\sim 50 \%$ of non-pregnant control capacity by the end of pregnancy [28]. Expression of Ugt2b1 is correspondingly reduced as well [28]. In addition to a decrease in the overall rate of glucuronidation, females are deficient in their ability to excrete newly conjugated BPA into the biliary system, likely due to reduced hepatic expression of the sinusoidal glucuronide transporter MRP2 [30].

Early investigations into the distribution of BPA in pregnant rodents following a single oral dose $(10 \mathrm{mg} / \mathrm{kg})$ showed that within an hour BPA was readily detectable in all of the maternal bodily compartments, as well as in fetal tissue [31]. The same effect also occurs with slightly lower doses $(2 \mathrm{mg} / \mathrm{kg}$ ) [32]. Studies using uterine perfusion or higher doses show that BPA is effectively transferred across the placental barrier into fetal circulation $[33 ; 27 ; 34 ; 35]$. Transfer of conjugated BPA across the placental barrier may be an active process. Nishikawa et al., hypothesized that the process may be mediated through the combined efforts of the organic anion-transporting polypeptide 4a1 (Oatp4a1) and multidrug resistance-associated protein 1 (Mrp1), both of which are known to be involved in the transplacental transport of endogenous conjugated steroids such as $17 \beta$-estradiol-glucuronide [27]. Much as it does in adults, BPA accumulates in the fetal liver in the hours following administration [35]. Unlike in adults, the expression of Ugt2b1 is extremely low in fetal tissue, meaning that fetuses are severely impaired in their ability to effectively deactivate bioactive BPA [27]. Moreover, expression of $\beta$-glucuronidase, an enzyme that deconjugates BPA-glucuronide, is quite high in the fetus and can explain the previous observations of higher than expected levels of bioactive BPA in fetal tissue [27]. The pharmacokinetic differences between fetal and adult animals supports the possibility that low doses of BPA—easily dealt with by the adult metabolism - may expose developing animals to pharmacologically relevant doses of active BPA. 
Following birth, placental transfer of BPA ceases to be a concern for the offspring. Nevertheless, the offspring's continued dependence on maternally-derived nutrition during lactation considerably extends its period of vulnerability. Low-dose BPA administered both orally and intraperitoneally can be subject to lactational transfer [36; 37]. One hour following a maternal dose of $100 \mu \mathrm{g} / \mathrm{kg} \mathrm{d} \mathrm{d}_{6}$-BPA, labelled total BPA concentration in the milk was markedly reduced compared to its serum levels [38]. Interestingly, the relative concentration of unconjugated BPA in milk was, on the average, 10-fold higher than what was found in maternal serum [38]. This phenomenon has also been observed in studies using intravenous infusions of a higher concentrations (0.13-0.54 mg/h) of BPA, with milk concentrations of unconjugated BPA found to be over twice those found in serum [39]. In both cases, this effect is likely due to the ease with which the lipophilic free BPA partitions into milk, compared to the more polar glucuronidated species [38]. This is an important consideration for low-dose toxicological studies, because pups may be exposed to a higher dose of free, bioactive BPA than expected based on maternal serum concentrations.

\subsection{PHARMACOLOGICAL TARGETS OF BPA}

BPA has traditionally been classified as a xenoestrogen: an exogenous substance with primarily estrogenic effects. In the past decade, the range of pharmacological targets of BPA has dramatically expanded in light of in-silico modelling of BPA-receptor interactions, as well as high-throughput screening efforts by toxicological authorities (i.e. [40]). BPA is now known to interact with a number of different receptors and enzymes, sometimes demonstrating affinities for these equal to or exceeding what is seen with the classical estrogen receptors. As such, any given BPA-induced phenotype should not be expected to be explainable entirely in terms of estrogenic stimulation. With increased attention to BPA's non-estrogenic effects, it may become possible to account for its famous ([41; 42]) non-monotonic dose-response curve. Non-monotonic dose-response curves often arise in situations where two or more separate species of receptor, usually with opposing physiological effects, respond at differing affinities to a single ligand. While the experiments described in this thesis are centred around BPA's estrogenic effects, a survey of some of its other metabolism-relevant receptor targets offers a wider perspective.

\subsubsection{Estrogen Receptors}

The existence of a receptor for estrogen could be inferred from early radioligand binding studies carried out in uterine and pituitary tissue. These experiments strongly suggested the existence of some substance that can, in a cell-type specific manner, selectively bind to and accumulate estrogen [43]. Later this property was attributed to what 
was called at the time the Estrogen Receptor (ER) [44]. This receptor was cloned more than a decade later [45]. When a second nuclear receptor for estrogen was discovered, it was named $\operatorname{ER} \beta$ [46], and the original estrogen receptor was retroactively named ER $\alpha$. The first ER $\alpha$ knockout mouse was generated in 1993 [47]. Surprisingly ${ }^{3}$, this mouse could survive and grow to adulthood, albeit with infertility [47]. Knockout mice were generated a few years after the discovery of $\operatorname{ER} \beta$, and study of these animals showed that it was somewhat dispensable for female reproductive function: knockout females could become pregnant, but their litters were smaller on the average than wild-type animals [48].

Ligand binding to either of these receptors induces in the receptor-ligand complex a number of conformational changes and post-translational modifications, the sum of which bring about nuclear translocation of the complex and subsequent binding to estrogen responsive elements (EREs) in the promoter regions of estrogen-responsive genes [49;50]. The structure of ER $\alpha$ is fairly well understood and can be divided into three major functional domains. The $\mathrm{N}$-terminus of the protein contains a region (AF-1) that is capable of ligand-independent transactivation of gene expression. This is followed by a highly conserved zinc finger containing DNA-binding domain, and finally a ligand-binding domain (LBD) toward the C-terminus. The LBD also contains a second transactivator domain known as AF-2 . In contrast to AF-1, AF-2 is liganddependent and responsible for the majority of ER $\alpha$ 's strongest genomic effects [49].

In 1936, Dodds \& Lawson showed that BPA is capable of acting in an estrogenic capacity in ovariectomized rats [51]. Given that BPA does not possess the chemical structure typical of endogenous sex steroids [51], it has historically been somewhat unclear exactly how it exerted its estrogenic effects. It might be reasonably assumed that BPA interacts with the ERs, but its unusual structure means that this cannot be guaranteed without careful pharmacological investigation. Early studies into the matter using MCF-7 cell cultures found that BPA was capable of inducing progesterone receptor expression with a potency roughly 2000-fold less than estradiol [5]. The ability of Tamoxifen to block this effect confirmed that it was due to what was then simply called the estrogen receptor, but is now known to be ER $\alpha$ [5].

The spectrum of BPA's biological activity is not quite coextensive with full ER $\alpha$ ligands such as estradiol, nor can it be accurately described as an ER $\alpha$ antagonist. In a model using HeLa cells transfected with a human ER $\alpha$ reporter gene, BPA alone acted as an agonist beginning at a concentration of $10^{-9} \mathrm{M}$, and at $10^{-6} \mathrm{M}$ showed agonism that was equivalent to estradiol at $10^{-8} \mathrm{M}$, and similar results were also obtained with $\mathrm{ER} \beta$ [52]. When BPA and estradiol were co-administered to ER $\alpha$-expressing cells, the net result was lower than expected reporter gene expression, suggesting that BPA can act in

\footnotetext{
${ }^{3}$ It had been assumed, at least before 1993, that since there were no known loss of function mutations in the human estrogen receptor gene (yet many such mutations in the androgen receptor gene), that this receptor was absolutely crucial for life.
} 
both an agonistic and antagonistic manner on $\operatorname{ER} \alpha$ but not $\operatorname{ER} \beta$ [52]. This finding was subsequently replicated with the same doses of BPA and estradiol in 293T and Hec-1 cell lines transfected with human ER $\alpha$ or ER $\beta$ luciferase reporter gene systems [53]. In contrast, BPA and estradiol were estrogenic both alone and together in the human osteosarcoma cell line HOS-TE85, indicating that the interaction between the two is antagonistic only under certain contexts [53]. BPA can act as a partial agonist of ER $\alpha$ transactivation in MCF-7 and HeLa based ERE reporter gene assays, but a full agonist on MCF-7 cell proliferation [50]. BPA's effects on intact animals are also different from those of estradiol. For example, unlike estradiol, BPA at $5 \mathrm{mg} / \mathrm{kg}$ does not affect uterine weight in neonatal rats and actually decreases peroxidase activity, but similar to estradiol it does cause a mild upregulation of the progesterone receptor [54]. When coadministered with an effective dose of estradiol, the net result is an increased uterine weight, but peroxidase activity and progesterone receptor expression that are lower than that seen in animals treated with estradiol alone [54]. Compounds that behave in this manner are known as Selective Estrogen Receptor Modulators (SERMs) [55].

The complex interaction between BPA and $\mathrm{ER} \alpha$ has recently been investigated in more exacting detail. Delfosse et al., used HeLa cells transfected with a modified version of $\mathrm{ER} \alpha$ lacking the $\mathrm{AF}-1$ region. BPA-induced transactivation was substantially reduced in these cells, as was its proliferative effect [50]. Because AF-1 is believed to be ligand-independent, its role in BPA-induced transactivation may account for why BPA often has effects that do not entirely match, or in some cases oppose, those of the receptor's endogenous ligands. The authors were also able to crystallize a complex containing the $\mathrm{ER} \alpha \mathrm{LBD}$ and $\mathrm{BPA}$, showing via crystallography that BPA assumes a binding mode that is quite similar to estradiol [50]. The BPA-ER $\alpha$ interaction involves 42 van der Waals interactions, in contrast with the 51 seen with estradiol. This, and a number of other differences in receptor geometry account for the reduced affinity of BPA for ER $\alpha$. There is also an important role for the hydrogen bonding that occurs between the dual phenol rings of BPA and a series of three polar residues buried within the LBD [50]. Since the dual phenol ring structure is common to all of the other bisphenols offered as replacements for BPA, it is reasonable to assume that they possess some level of affinity for $\mathrm{ER} \alpha$ as well, and thus their presumed safety may be unjustified. Contrary to the assumptions of earlier investigations, the classical estrogen receptors are not confined strictly to the intracellular or intranuclear space. Rather, there is a growing body of evidence suggesting that these receptors can also be localized to the plasma membrane $[56 ; 57 ; 58 ; 59 ; 60]$, and exert rapid effects on the MAPK/ERK and PI3K pathways when so configured. This pathway is important for the regulation of female sexual receptivity, as well as the synthesis of neuroprogesterone in cycling females [61]. Membrane estrogen signalling also appears to be important in the neuroprotective effects of estradiol [62]. The means by which ER $\alpha$ is trafficked to the membrane are presently unknown, but the process depends on 
post-translational modification of the ER $\alpha$ protein by S-palmitoylation by the palmitoylacyltransferase enzymes DHHC-7 and DHHC-21 [63; 64]. The possibility that BPA exerts its effects through membrane ERs has received only limited attention. In part, this is due to the fact that BPA interacts with several membrane receptors, and the absence of specific antagonists for each makes their roles difficult to disentangle. Often, interactions between BPA and membrane-bound ER $\alpha$ are simply inferred by the rapid activation of MAPK/ERK signalling (e.g. [65]).

The affinity of BPA for the classical ERs relative to their endogenous steroid ligands seems to be vanishingly small and it is therefore difficult to accept as a source of serious upset against the hormonal milieu present in normal physiology. This is doubly so given the extremely small concentrations of BPA typically found in living systems. It may be beneficial, given this, to cast a wider net for possible BPA receptors. The estrogen-related receptors (ERRs) are a recently characterized subfamily of nuclear receptors with no known endogenous ligands. The family comprises ERR $\alpha$, $\operatorname{ERR} \beta$, and the most recently discovered ERR $\gamma$ [66]. ERR $\gamma$ is expressed in the mouse as early as E11, with expression continuing into adulthood in the brain, heart and kidney [66]. ERR $\gamma$ does not bind with estradiol nor any of the other typical sex steroids [67]. It does, however, bind readily with BPA at nanomolar concentrations [67]. Numerous structural studies have confirmed BPA is a very high affinity ligand for ERR $\gamma$ [68; 69; 70; 71]. Still, because the normal physiological function of ERR $\gamma$ remains ill-defined, it is difficult to predict what effects might be expected of a in-vivo interaction between it and BPA. The fact that ERR $\gamma$ is constitutively active and requires no ligand for transcriptional activity [66] might lead to the hypothesis that BPA acts as an inverse agonist. However, this does not appear to be the case. Takayanagi et al., used a luciferase reporter gene assay in HeLa cells and found that BPA does not affect ERR $\gamma$ constitutive transactivation at any dose, but that it is capable of blocking the inverse agonism of 4-hydroxytamoxifen [67]. More recently, BPA was shown to interfere with otolith formation in a zebrafish model, and this interference could be blocked by morpholino knockdown of ERR $\gamma$ or recapitulated in the absence of BPA by over-expression of ERR $\gamma$ [69]. These results notwithstanding, the physiological significance of ERR $\gamma$ as a target for BPA remains largely unexplored, and this will likely continue until an endogenous ligand is discovered or mammalian knockout models are studied in more depth.

BPA has a range of possible targets that is not limited solely to nuclear receptors and their membrane-bound variants, although these are the best understood. GPR30, a $G$ protein-coupled receptor, was originally discovered as an orphan receptor, but eventually revealed to be involved in a number of physiological functions [72]. Stimulation of this receptor by estradiol leads to rapid, non-genomic effects including the mobilization of intracellular $\mathrm{Ca}^{2+}$ and activation of the PI3K signalling pathway [73; 74]. BPA demonstrates a relative binding affinity for GPR30 that is between 8-50 times 
greater than that of the classical ERs [75]. This leaves its potency at levels that are still well below those of the endogenous estrogens, though it does suggest that GPR30 is an important target for BPA. BPA binding to GPR30 activates the downstream second messengers cyclic adenosine monophosphate (cAMP) and phosphorylated extracellular signal-regulated kinase $1 / 2$ (pERK1/2) [75; 76], and it can therefore be taken to function as an agonist in this context. This phenomenon is frequently cited in BPA research, though rarely addressed in the course of in-vivo experimental procedures. At present, the only study to do so failed to show a major role for GPR30 in the ability of BPA to regulate aromatase expression in the zebrafish brain, though this is but one potential assay out of many [77].

\subsubsection{Androgen Receptor}

When research began on androgen function, an early question was whether testosterone and its $5-\alpha$ reduced metabolite dihydrotestosterone (DHT) interacted with differing affinities at the same receptor, whether they had separate receptors, or whether testosterone even had a receptor in the first place. DHT, a much more potent androgen than testosterone, was known to be produced locally in androgen-sensitive tissues, and so the issue of a receptor for testosterone was difficult to disentangle [78]. Radioligand studies using $\left[{ }^{3} \mathrm{H}\right]$-testosterone in rat prostate tissue demonstrated the existence of a nuclear protein capable of binding both testosterone and DHT, though the latter was invariably present in higher concentrations due to local $5-\alpha$ reductase activity [79; 80$]$. Subsequent study of non-prostate tissue demonstrated that conversion to DHT was not required for the androgenic effects of testosterone, and it was very likely that there was only one variety of androgen receptor [81]. The Androgen Receptor (AR) was later cloned, and found to be located on the X chromosome [82]. The AR belongs to the steroid hormone group of nuclear receptors, possessing a high degree of similarity to the classical ERs. Once bound with a ligand, the AR translocates to the nucleus, forms homodimers and effects transcription at Androgen Response Elements (AREs) in the promoter regions of androgen-responsive genes [83].

The chemical differences between the endogenous androgens and estrogens are sufficient to preclude any noticeable crosstalk at their respective receptors, which have presumably evolved to discriminate between closely related hormones with a high degree of precision. Such discrimination is often not possible with endocrine disruptors, which may possess chemical characteristics that are somewhat ambiguous from the receptor's point of view. For this reason, the androgen receptor has recently come into focus as a potential target for BPA. BPA has been reported to possess dose-dependent antiandrogenic activity, as measured in a yeast reporter gene assay [84]. Moreover, BPA at $10 \mu \mathrm{M}$ is capable of inhibiting testosterone-induced nuclear translocation of the AR [84; 85], as well as AR transactivation at the Prostate-Specific Antigen (PSA) promoter 
[84]. This has since been replicated numerous times in bacteria, yeast, and mammalian reporter gene systems [86; 87; 85]. Increasing the concentration of DHT from $100 \mathrm{nM}$ to $10 \mu \mathrm{M}$ in the medium seems to compensate for the inhibitory effect of BPA, suggesting that BPA is a competitive antagonist at the AR [86]. In further support of this, it has been shown in several studies that BPA is capable of dose-dependently displacing the radioactively labeled agonist R1881 bound to the AR [88; 89; 85].

The situation becomes more complex when in-vivo systems are studied. Similar to how the interactions between BPA and ER $\alpha$ vary between cell lines and organs under study, it might be expected that BPA-AR interactions vary as well. Research into this area is comparatively scarce, but the potential is highlighted by a recent study in zebrafish. This study found that precocious neurogenesis and adult hyperactivity induced by low doses of BPA during the embryonic stage could be blocked by inhibition of the AR with flutamide, but not with ER inhibition using ICI 182,780 [90]. These results suggest that BPA can also act as an AR agonist, perhaps in a tissue-specific manner. As the authors found, pharmacological inhibition of aromatase was also successful in blocking the effect. The aromatase promoter contains AREs, allowing for its transcriptional regulation by AR. Indeed, it seems that in the zebrafish model AR-induced aromatase upregulation is critical for the developmental effects of BPA [90; 77]. This finding has important implications for the understanding of BPA as an estrogenic substance, for it may be that it can overcome its own feeble affinity for classical ERs by stimulating the body to produce more endogenous estradiol. The fate of the putative excess estradiol remains to be accounted for, since the phenotype was not disrupted by inhibition of the classical ERs. The balance sheet may be further altered by the fact that excess aromatase activity may consume precursors that could otherwise have formed DHT, or some other steroid metabolite. More research is needed to clarify the nature of this effect and whether it is also of relevance in mammalian models.

\subsubsection{Peroxisome Proliferator-Activated Receptor $\gamma$}

Under certain conditions, cells of the 3T3-L1 mouse fibroblast line will differentiate into adipocytes and begin accumulating triglycerides [91]. Because this conversion can be stimulated by adipogenic factors such as insulin [91], this cell line is often used as an assay for compounds that stimulate adipogenesis. The Peroxisome ProliferatorActivated Receptors (PPARs) are critical in the induction of adipose tissue. As is often the case, the discovery of the PPARs was the result of probing for the molecular targets of compounds already known to exert a certain physiological effect. In this case, the compound in question was nafenopin, one of many found to be capable of increasing the number of hepatic peroxisomes, reducing plasma triglyceride levels and increasing hepatic $\beta$-oxidative capacity [92]. Early radioligand binding studies using $\left[{ }^{3} \mathrm{H}\right]$-nafenopin revealed the presence a cytosolic protein in liver cells for which it is a 
ligand [92]. Subsequent study found that the PPARs are members of the highly conserved family of nuclear receptors to which the ERs, AR, and more also belong [93; 94], and still later investigation revealed the existence of a number of PPAR subtypes [95]. Among these, PPAR $\gamma$ appears to be the most relevant for the adipogenic effects of endocrine disruptors such as BPA.

The PPARs are structurally and functionally similar to other nuclear receptors, and thus exert their effects primarily through the control of gene transcription. After binding with an agonist PPAR $\gamma$, translocates to the nucleus and dimerizes with members of the Retinoid X Receptor (RXR) family, whereupon the complex, in concert with various coactivators increases transcription of genes containing PPAR response elements (PPREs) [96; 97; 98]. Aside from the thiazolidinedione (TZD) class of anti-diabetic agents, certain herbicides, fungicides, phthalates, flame retardants, as well as industrial plasticizers are all capable of binding to PPAR $\gamma$ and modulating its transcriptional activity. In the midst of this apparent promiscuity to exogenous agents, the endogenous ligand for PPAR $\gamma$ is not yet known with certainty. Fatty acids and certain fatty acid metabolites bind to and activate PPAR $\gamma$, and for this reason lipid sensing is its most plausible physiological function [99]. A number of genes are the transcriptional targets of PPAR $\gamma$, and these are active during development to regulate initial adipogenesis [100]. Acting as it does as a transcriptional regulator of lipogenesis, PPAR $\gamma$ signalling remains important later in life as a regulator of adipose tissue metabolism [101;102; 94].

BPA $(20 \mu \mathrm{g} / \mathrm{ml})$ potentiates the insulin-induced conversion of 3T3-L1 cells to adipocytes, as measured by increased lipoprotein lipase (LPL) and glycerol-3-phosphate dehydrogenase (GPDH) activity, as well as increased triglyceride accumulation [103]. This effect appears to be dependent on the Phosphatidylinositol 3-Kinase (PI3K) pathway, as it can be blocked by the PI3K inhibitor LY294002 [104]. BPA at $80 \mu \mathrm{M}$ can induce the expression of numerous markers of adipocyte differentiation including PPAR $\gamma 2$, fatty acid synthase (FAS), and leptin in 3T3-L1 cells [105]. This study used a relatively short time course, and it is perhaps for this reason that there was no evidence of increased triglyceride synthesis [105]. The in-vivo research conducted so far has yielded similar results with respect to adipogenesis. Female rat pups perinatally exposed to BPA at an estimated $70 \mu \mathrm{g} / \mathrm{kg} /$ day show an increase in white adipose tissue mass at $\mathrm{P} 21$, as well as increased expression of the adipogenic markers CCAAT/enhancerbinding protein $\alpha(\mathrm{C} / \mathrm{EBP} \alpha)$, PPAR $\gamma$, sterol regulator element-binding protein $1 \mathrm{C}$ (SREBP-1C), LPL, and FAS [106]. The fact that this phenotype appeared to be specific to females suggests an effect of sex, at least in the biological context of a whole animal $^{4}$.

Many of the adipogenic genes known to be up-regulated by exposure to BPA are

${ }^{4}$ The 3 T3 cell line was originally isolated from Swiss mouse embryos [107], and its genetic sex remains uncertain. Though sex steroids are not part of the standard differentiation medium for 3T3-L1 cells, they do elicit a response [108]. 
themselves transcriptional targets of PPAR $\gamma$, and this circumstantial evidence justifies a closer inspection of the possibility that BPA is a PPAR $\gamma$ ligand. In-silico modelling of BPA-PPAR $\gamma$ interactions suggests that while BPA can act as a ligand for PPAR $\gamma$, its affinity is quite low compared to ER $\alpha$ and other known nuclear receptor targets [71]. The issue has recently been explored more directly by two separate in-vitro studies using reporter genes to measure PPAR transcriptional activity. Pereira-Fernandes et al., the more recent of the two, used specially prepared human osteoblast cells transfected with a luciferase reporter construct under the control of the PPRE. This study found that BPA at 10 and $50 \mu \mathrm{M}$ leads to an increase in PPAR $\gamma$ transcriptional activity [109]. These results are in contrast to an earlier study by Riu et al., which found no effect of BPA in PPAR $\gamma$ transcriptional activity in HeLa cells [110]. The two-hybrid screening system used by these authors may account for the discrepancy. This system, described in [111], did not directly measure the transcriptional activity of complete PPAR $\gamma$ at the PPRE, rather it used a fusion protein containing the yeast activator GAL4 and the PPAR ligand-binding domains to induce transcription of luciferase linked to the GAL4 activator [111]. This approach has the effect of removing a great deal of the ordinary physiological context that surrounds this signalling pathway. This is useful insofar as it greatly simplifies the relationship between BPA and PPAR $\gamma$ per se, but both the cellular context and the presence of promoter-specific transcriptional enhancers or repressors has a very large effect on nuclear receptor function ${ }^{5}$, and given this a study using the complete PPAR $\gamma$ protein and PPRE site is preferable.

While the BPA-PPAR $\gamma$ relationship may be a key player in mediating BPA's developmental effects, the pathway as a whole also holds other interesting possible targets for BPA. The advantage of the approach used by Pereira-Fernandes et al., is the ability to study the pathway in terms of its end consequences (i.e. transcriptional activity at the PPRE). Transcriptional activity at the PPRE actually involves a heterodimeric complex of ligand-bound PPARs and RXRs [96; 97; 98]. RXR $\alpha$ shows promise as a BPA-sensitive receptor, with in-silico estimated affinity nearly matching that of ER $\alpha$ [71]. Moreover, BPA showed potent concentration-dependent RXR agonism in a yeast two-hybrid screening assay but only when co-incubated with a rat liver S9 fraction, indicating that a BPA metabolite is likely responsible for this effect [114]. Research into the possibility that BPA induces adipogenesis through interactions with $\mathrm{RXR} \alpha$ remains in its early stages, but it is a promising direction.

\footnotetext{
${ }^{5}$ In many cases, the broader context of gene transcription is beyond the scope of neuroscientific or even toxicological studies concerned with risk assessment, but it is of critical importance in tumour endocrinology. As an example, ER $\alpha$ positive breast cancers often develop tamoxifen resistance, and in the search for a mechanism researchers have invoked ER splice variants, GPR30, insulin-like growth factors, epigenetic imprints, microRNAs, and the complex interplay of transcriptional co-repressors and co-activators, and yet the phenomenon remains mysterious [112; 113].
} 


\subsubsection{Glucocorticoid Receptor}

The glucocorticoids are pleiotropic steroid hormones secreted by the zona fasciculata of the adrenal cortex. The glucocorticoid receptor (GR) is a nuclear receptor that is expressed in virtually every cell type throughout the body. Because each variety of cell can mount a unique response to glucocorticoid stimulation, the effects of these hormones can be extremely diverse, ranging from the metabolic, immunologic, to homeostatic and beyond. The glucocorticoids can influence the development of many organ systems, as is often demonstrated in models of low birth weight and prenatal stress (for review, see [115]).

One area of particular interest for the topic at hand is the role of glucocorticoids in adipose tissue development. Adipocyte differentiation normally requires glucocorticoid receptor activity in order to proceed. Atlas et al., found that in spite of this requirement, BPA on its own can stand in for the synthetic glucocorticoid dexamethasone in promoting lipid accumulation in the 3T3-L1 model of adipocyte differentiation [116]. BPA at concentrations as low as $0.1 \mathrm{nM}$, and as high as $10 \mathrm{nM}$ can induce adipocyte protein 2 (aP2) and adipsin protein expression in 3T3-L1 cells in the absence of dexamethasone, but the two together can synergistically induce even larger effects on those proteins [116]. Wang et al., found, using fat biopsies obtained from human children, that BPA concentrations as low as $10 \mathrm{nM}$ cause up-regulation of $11 \beta$-hydroxysteroid dehydrogenase (11 $\beta$-HSD), PPAR $\gamma$, and LPL [117]. The BPA-induced up-regulation of $11 \beta$-HSD was blocked by co-administration of both RU486, a GR antagonist and CBX, an $11 \beta$-HSD inhibitor [117].

These results seem to imply that BPA can interact with the GR, and indeed recent in-silico modelling studies bear this out showing that BPA can bind with GR, though with a lower free energy of binding than either cortisol or dexamethasone [118]. Still, BPA must differ from traditional agonists in some way. Atlas et al., found that BPA at effective doses does not induce nuclear translocation of GR in COS-7 cells, nor does it induce GR transactivation in NIH-3T3 cells at either glucocorticoid response element (GRE)-containing promoters or the glucocorticoid-responsive mouse mammary tumour virus (MMTV) promoter [116]. BPA does, however, potentiate the transcriptional activity of CCAAT enhancer binding protein $\delta(\mathrm{C} / \mathrm{EBP} \delta)$ in the presence of GR, suggesting that the two may act as a transcription factor complex that is susceptible to BPA, and the transcriptional targets of this complex may explain the specificity of BPA's observed effects [116]. This may vary between models, as Sargis et al., used 3T3-L1 cells transfected with a luciferase construct linked to the GRE to explore the potential effects of this interaction. These cells showed a marked increase in GRmediated luciferase expression when exposed to BPA at $1 \mu \mathrm{M} / 1$, an increase that was nearly equivalent to that induced by dexamethasone [119]. Still, this level of agonism was insufficient to substitute for the effects of stronger glucocorticoids in inducing 
adipogenesis and promoting lipid accumulation [119]. Here it seems that there is a difference between full engagement of the adipogenic program with the lipid accumulation and transcriptional changes thereby entailed, and activation of a subset of its transcriptional markers. Atlas et al., and Wang et al., found that BPA could stand in for stronger GR agonists in a number of GR-induced transcriptional markers [116; 117], but Sargis et al., found that lipid accumulation itself was not amenable to such substitution [119]. Since the putative in-vivo situation would naturally include endogenous glucocorticoids, the fact that BPA cannot act as a full and complete GR agonist on its own is not necessarily exonerating, given that it does seem to have a synergistic effect with full GR agonists [116;119].

\subsection{EFFECTS OF BPA ON SEXUALLY DIMORPHIC HYPOTHALAMIC NUCLEI}

BPA is active at a number of steroid receptors, and beyond this it can affect the expression and function of enzymes involved in steroid biosynthesis and metabolism (see Section 1.3). Although BPA has a diverse range of pharmacological targets, understanding it as a primarily estrogenic substance produces a number of testable hypotheses that are highly relevant to its effects on human health and development. Chief among these is the possibility that BPA interferes with the sexual differentiation of the brain.

The sexually dimorphic nucleus of the preoptic area (SDN-POA) is substantially larger in males than in females, with a difference of three to eight fold being the figure usually given [120]. Males and females are born with the same number of cells in this nucleus, but the influence of neonatal estradiol-aromatized from testicular testosterone-inhibits apoptosis in males, and so as development runs its course females lose a sizeable number of cells in this region [121]. Because the sexually dimorphic nature of this nucleus is so pronounced (sufficient, indeed, for it to have a name that advertises this fact), it is reasonable to expect large and easy to detect effects from interventions that affect masculinization. In an early study, Nagao et al., exposed neonatal rat pups to subcutaneous injections of BPA at $300 \mu \mathrm{g} / \mathrm{g}$ from P1-5. An extensive analysis of reproductive function found that while the positive control estradiol benzoate severely hampered reproductive behaviour in both sexes and reduced the volume of the SDN-POA in males, BPA had essentially no effects on these measures [122]. Using a more chronic model of exposure, Kown et al., examined behavioural and neuroanatomical outcomes in the female offspring of rats exposed to varying doses $(3.2,32$, and $320 \mathrm{mg} / \mathrm{kg}$ by oral gavage) from G11-P20. Behavioural and physiological outcomes were not affected by BPA in this study: females showed the expected rates of lordosis, estrous cyclicity, and pubertal timing. Moreover, the volume of the SDN-POA was not affected by BPA, though it was increased by DES, which was given at $15 \mu \mathrm{g} / \mathrm{kg} /$ day as a positive control [123]. 
The situation has become more complicated in recent years, as authors turn toward quantifying immunohistochemically-defined nuclei. For example, He et al., administered BPA by oral gavage to pregnant and lactating rats (G6-P21) at $2.5 \mu \mathrm{g} / \mathrm{kg} / \mathrm{day}$ and found an increased volume of the SDN-POA in male but not female rats, as measured by calbindin-D28k immunofluorescence [124]. Estradiol again played the part of a positive control, and as usual it was able to produce the expected masculinization of the SDN-POA in females [124]. Similarly, BPA was found to increase the number of calbindin-D28k cells in the SDN-POA of males exposed to BPA at $500 \mu \mathrm{g} / \mathrm{day}$ from P1-2 [125]. Most recently, McCaffrey et al., found that males exposed perinatally from G12-P10 to BPA at a variety of doses from $10-10,000 \mathrm{ng} / \mathrm{kg} /$ day all showed reductions in the volume and cell density of the SDN-POA, as measured by unbiased stereology on calbindin-D28k immunolabelled neurons [126]. It seems that depending on the exact circumstances under which it is administered, and likely also the sensitivity of the animal model being used, BPA either increases, decreases, or does nothing to the size of the SDN-POA in male or female animals. Some part of this variability may come from the histological approach chosen to measure the nucleus. While the calbindin-D28k compartment is smaller than the whole nucleus as measured by Nissl staining, its borders are very clear and may allow for more reproducible measurements $[127 ; 128]$.

As with the SDN-POA, male and female rodents are born with the same number of neurons in the Anteroventral Periventricular nucleus (AVPV). This quickly changes as the process of sexual differentiation comes online, in this case preventing apoptosis in females and promoting it in males [129]. The possibility that perinatal BPA exposure masculinizes the AVPV was examined by Rubin et al., who exposed pregnant and lactating mice to BPA using subcutaneously implanted osmotic minipumps [130]. These pumps delivered a constant infusion of BPA at doses of either $25 \mathrm{ng} / \mathrm{kg} / \mathrm{day}$ or $250 \mathrm{ng} / \mathrm{kg} /$ day. As expected, control females had significantly more tyrosine hydroxylase (TH) immunopositive neurons in the AVPV than control males. This sex difference was attenuated by the $25 \mathrm{ng} / \mathrm{kg} /$ day dose, and virtually absent at the 250 $\mathrm{ng} / \mathrm{kg} /$ day dose [130]. This finding was later replicated in rats by McCaffrey et al., who showed that BPA caused a reduction in the number of tyrosine hydroxylase (TH) immunopositive neurons in the AVPV, with the lowest dose of $10 \mu \mathrm{g} / \mathrm{kg} /$ day bringing female values within the same range as those of control males, implying a masculinized phenotype [126]. The effect was present, though somewhat less pronounced with each of the higher doses. Male rats exposed via maternal subcutaneous injection of BPA at $2 \mu \mathrm{g} / \mathrm{kg} /$ day from G10-P7 showed increased numbers of kisspeptin immunopositive neurons in the AVPV, approaching levels normally seen only in control females [131]. The duration and mode of exposure seems important here, as another study which exposed neonatal female rats to BPA at $50 \mu \mathrm{g} / \mathrm{kg} /$ day for the first four days of life found no effect of that dose on kisspeptin fibre density in the AVPV or arcuate nu- 
cleus (ARC) [132]. The higher dose, a comparatively enormous $50 \mathrm{mg} / \mathrm{kg} /$ day used by this study did produce modest decreases in both measures, however [132].

Adewale et al., explored several other hypothalamic sexual dimorphisms using female rat pups exposed to BPA at $50 \mu \mathrm{g} / \mathrm{kg}$ or $50 \mathrm{mg} / \mathrm{kg}$ by subcutaneous injection on P0-P3. Adult females exposed to both doses of BPA, as well as estradiol benzoate $(25 \mu \mathrm{g})$ showed increased numbers of oxytocin immunopositive cells in the paraventricular nucleus (PVN) [133]. Estradiol benzoate was used as a positive control in this study, and in fact it could induce a number of other effects that BPA was generally unable to match. Among these was an increased in serotonin (5-HT) fibre density in the ventromedial hypothalamus (VMH), and a decrease in the number of ER $\alpha$ immunopositive cells in the medial preoptic area (MPOA), both of which are characteristic of the masculinized brain [133].

Taken together, these studies seem to be plagued by contradictory findings, and rendered nearly incommensurable by methodological differences. Positive controls, when included, typically produce the expected effects regardless of methodological difference, and this fact complicates the issue. It may be that the systems and processes upon which BPA acts are different from those targeted by the estrogenic substances typically used as positive controls. If this is true, then a careful assessment of dose timing, as well as an exhaustive survey of the dose-response relationship will be key, as many studies may simply miss the period of vulnerability or vastly overshoot BPA's range of biological activity.

\subsection{OBESOGENIC EFFECTS OF BPA}

While the earliest studies of the developmental effects of BPA were concerned primarily with reproductive, neurobehavioural, and oncogenic outcomes, with measures and protocols suited to the expected effect of estrogenic interference, in the midst of these there were occasionally observations of altered body weight. Not being the primary focus of such studies, these observations were often relegated to the category of interesting yet incidental observations.

One of the earliest studies into the consequences of perinatal BPA exposure on adult prostate weight was carried out by Nagel, vom Saal, et al., [134]. These authors found increased prostate weight and body weight in adult males exposed maternally from E11-17 to BPA at $2 \mu \mathrm{g} / \mathrm{kg} /$ day [134]. In another early study, female mouse pups exposed prenatally to low $(2.4 \mu \mathrm{g} / \mathrm{kg})$ doses of BPA were heavier on average than control mice, and the magnitude of this effect increased in proportion to the number of females around which they were positioned in-utero [135]. Thus females positioned between two other females in-utero tended to show stronger responses to BPA when compared to control females with the same in-utero position. Rubin et al., exposed pregnant rats to BPA via drinking water at doses of 1 and $10 \mathrm{mg} / \mathrm{L}$ from 
G5 until weaning. The authors estimated that dams in these two groups received an average dose of 0.1 and $1.2 \mathrm{mg} / \mathrm{kg} /$ day respectively. In this study, pups exposed to both doses of BPA had higher body weights than controls at all time-points studied, abnormal estrous cycles in adulthood, and hyposecretion of LH [136]. Differences in body weight persisted through adulthood, though they remained more pronounced in females [136]. Similar effects were replicated by Nikaido et al., using higher doses of BPA (0.5 and $10 \mathrm{mg} / \mathrm{kg}$ ) administered subcutaneously to dams from G15 until weaning [137]. While the majority of these studies have been carried out during intervals spanning the perinatal period, one study has shown that BPA exposure prior to implantation can also exert long-lasting effects on body weight. Takai et al., exposed cultured mouse embryos at the morula stage to $1 \mathrm{nM}$ or $100 \mu \mathrm{M}$ BPA and found that while these two doses respectively increased and decreased embryo viability, when embryos were implanted into females, both had the effect of increasing animal body weight at weaning [138].

Miyawaki et al., carried out one of the first studies explicitly aimed at exploring the possible obesogenic effects of perinatal BPA in mice, exposing dams via drinking water (1 and $10 \mu \mathrm{g} / \mathrm{ml}$ from G10 until weaning). Sampling juveniles at PND30, the authors found that the effects of their BPA doses varied between the sexes, with the lower dose leading to increased body weight and adipose tissue weight in females, and only the higher dose affecting males in that regard [139]. Females exposed to the low dose also showed hyperleptinemia and increased total cholesterol relative to their controls. While the high dose rendered male mice heavier and fattier, this did not appear to have any serological correlate, as serum leptin, total cholesterol, triglycerides and glucose were all unchanged in these animals [139]. Somm et al., used rats in a similar model $(1 \mu \mathrm{g} / \mathrm{ml}$ BPA in drinking water from G6 until weaning) and demonstrated that female offspring rendered obese by BPA exposure had morphological and transcriptional changes in adipose tissue indicative of increased adipogenesis [106] (see section 1.3.3).

Perinatal exposure to BPA has also been linked to impairments in glucose homeostasis in adulthood. In one study, adult male mice exposed prenatally via maternal subcutaneous doses of either 10 or $100 \mu \mathrm{g} / \mathrm{kg}$ of BPA showed decreased glucose tolerance in a standard intraperitoneal glucose tolerance test [140]. Moreover, these animals displayed insulin resistance and alterations in pancreatic islet function [140]. A subsequent investigation using a wide range of doses $(5,50,500,5000$, and $50,000 \mu \mathrm{g} / \mathrm{kg} / \mathrm{day}$ via maternal oral exposure from G9-18) found increased fat pad mass, adipocyte number and volume in adult male offspring exposed prenatally to $5 \mu \mathrm{g} / \mathrm{kg} /$ day BPA [141]. Adult males from this study also showed impaired glucose tolerance and insulin tolerance [141]. Importantly, this study shows that prenatal exposure during the main period of adipogenesis is sufficient to bring about many metabolic impairments in adulthood. A similar model of exposure (10 $\mu \mathrm{g} / \mathrm{kg} /$ day subcutaneously from G9-16) found that exposed male mice had elevated body weight, perigonadal fat, and circu- 
lating non-esterified fatty acids (NEFAs) at 28 weeks of age [142]. This study weaned animals onto both a control and high-fat diet (HFD), and it was interesting to note that BPA-exposed animals, even when consuming a standard chow diet in adulthood, showed levels of obesity and metabolic impairment that approached that seen in HFD consuming animals [142]. Pancreatic islets isolated from BPA-exposed males at 17 weeks of age showed increased glucose-stimulated insulin secretion as compared to control animals, and these effects again approached those that were induced by HFD exposure alone [142].

On the other hand, Ryan et al., employed a very sophisticated and environmentally relevant study of the metabolic effects of perinatal BPA exposure in mice and found very limited evidence of an obesogenic effect at the dose they used (approximately $1 \mu \mathrm{g} / \mathrm{kg}$ via maternal oral exposure from G0-P21) [143]. Offspring from this study showed increased postnatal growth, but this levelled off quickly, and by adulthood there was no effect of BPA on body composition or weight [143]. The dose used by Ryan et al., is among the lowest examined thus far with respect to BPA's obesogenic effects, and may represent the low end of the dose-response curve. A study conducted in rats found evidence for an obesogenic effect of developmental BPA exposure at $50 \mu \mathrm{g} / \mathrm{kg}$ administered maternally via oral gavage. Offspring from this study were heavier than controls when fed a high-fat diet in adulthood, and progressively developed impairments in glucose tolerance that were amplified by exposure to the high-fat diet [144]. Interestingly, this study also used doses of $250 \mu \mathrm{g} / \mathrm{kg}$ and $1250 \mu \mathrm{g} / \mathrm{kg}$ and found no effects. Findings of this sort are called non-monotonic dose-response curves, and they are not uncommon in EDC research. Non-monotonic dose-response curves are found in situations where physiological effects can be observed in response to both high and extremely low doses, with a wide intermediate range wherein the substance in question has no apparent biological activity [41; 42]. This could explain the lack of effects observed by Wei et al., and other studies at higher doses. For example, two of the largest and most thorough toxicological investigations of BPA found no effect on body weight $[145 ; 146]$. These studies were carried out using a variety of doses, the lowest of which was still over $1000 \mu \mathrm{g} / \mathrm{kg}$ - apparently well within the intermediate zone of ineffectual doses. Wei et al., have continued to use this model of BPA exposure, and recently demonstrated that it predisposes animals to non-alcoholic steatohepatitis. In particular, male offspring of BPA-exposed dams showed increased liver triglyceride accumulation and free fatty acid content on both a standard and high-fat diet [147]. Placing animals on a high-fat diet had an additive effect with perinatal BPA exposure, leading to markedly elevated expression of the lipogenic genes Sterol regulatory element-binding transcription factor 1 (Srebf1), FAS, PPAR $\gamma$, and the gluconeogenic genes Glucose 6-phosphatase (G6pc) and Phosphoenolpyruvate carboxykinase 1 (Pck1). These were accompanied by morphological markers of steatosis, namely an increase in the size and number of lipid droplets in the liver and an increase in overall liver size [147]. 
Evidence for an effect of early-life BPA exposure on body weight and metabolism in adulthood is thus accumulating at a gradual rate. As of yet, there has been no neurological basis of action offered through which the aforementioned effects might operate. To be sure, BPA's actions on peripheral organs such as the adipose tissue and pancreas likely account for a number of its metabolic effects. Nevertheless, it is rare to observe a developmental model of obesity that does not also include among its phenotypic constituents some degree of altered brain function. The brain can be expected, through its bi-directional neural and hormonal interactions with the peripheral organs, to exaggerate or at least complement their pathologies.

\subsection{CHALLENGES IN ENDOCRINE DISRUPTOR RESEARCH}

The discovery that a certain widely used chemical is reliably associated with harmful effects in laboratory research is often sufficient to provoke a public outcry and corresponding legislative response ${ }^{6}$. This is already the case in Canada and the many other jurisdictions that have banned BPA-containing baby bottles from sale. Manufacturers often find reason to voluntarily remove from their formulations chemicals with a bad reputation, even if the balance of scientific evidence remains ambivalent on the subject. In any event, it is critical that research into EDCs be conducted with a great deal of attention to environmental relevance, replicability, and the removal of all plausible confounds.

\subsubsection{Modeling Oral Exposure}

Human exposure to BPA is primarily through the oral route. Though transdermal exposure to BPA is possible, the magnitude of this exposure is relatively small when compared with oral exposure. The United States National Toxicology Program (NTP) has published a comprehensive series of guidelines intended to address inconsistencies and sources of bias in endocrine disruptor research. First among these are the need for more in-vivo studies using oral routes of administration [148]. Owing to a substantial first-pass effect oral BPA exposure is taken to be less potent than parenteral routes on a gram for gram basis. Oral gavage does not provide a satisfactory solution to this problem from the point of view of either pharmacokinetics or ecological validity. Oral gavage constitutes a bolus administration of the compound in question, and this is not reflective of typical human patterns of food intake. Moreover, oral gavage of oil-based compounds is stressful, and this fact may produce unexpected confounds as well [149]. In our studies, experimental compounds will be incorporated into the

\footnotetext{
${ }^{6}$ Whether a legislative response takes place depends a great deal on the public perception of the compound in question. In the aggregate, the adverse effects of excess dietary sugar massively outweigh even the most generous estimates of BPA's effects, yet attempts to control levels of 'added sugar' through legislation have almost universally met with dead ends.
} 
animals' diet and consumed ad-libitum over the period of study, thereby approximating as fairly as possible the human conditions of exposure.

\subsubsection{Diethylstilbestrol, an Estrogenic Positive Control}

The second concern that is frequently raised by the NTP in response to the current BPA literature is the lack of appropriate positive controls [148; 150]. Many studies elect to use a potent positive control at a relatively high dose that can reliably produce a particular outcome, for example doses of estradiol sufficient to upset sexual development. While this is preferable to the complete exclusion of a positive control, it may be misleading in the context of endocrine disruptors which, as their name suggests, are expected to produce subtle disruptions in development, not massive upheavals. Even when much lower doses of substances chemically dissimilar to biological hormones are used, the use of a positive control is invariably theory-laden. That is, the experimenter's choice of a positive control involves assumptions about the pharmacological workings of the compound under study. Moreover, information gained from the inclusion of a positive control is only useful insofar as it can be safely assumed that the compound under study produces effects that are alike in kind, but perhaps different in degree from the pharmacologically similar positive control.

Diethylstilbestrol (DES, (E)-3,4-Bis(4-hydroxyphenyl)-3-hexene) is a synthetic estrogen first developed and characterized by Dodds in 1938 [151]. Competitive binding assays indicate that DES is at least as potent as estradiol [152], while the Yeast Estrogen Screen and the E-Screen assays suggest that DES is between 1.1-2.5 times as potent as estradiol [153]. DES possesses reliable estrogen-like activity in-vivo as well, with numerous studies showing that early-life exposure to DES masculinizes the female SDN $[154 ; 155 ; 156]$. Like BPA, DES has also been suggested to act as an obesogen. Exposing lactating CD-1 mice to DES at $1 \mu \mathrm{g} / \mathrm{kg}$ from P1-5 yielded female offspring that were heavier than their controls in adulthood, but the situation was reversed in male offspring, who were found to be lighter than their controls in adulthood [157; 158]. X-ray densitometry of DES-exposed animals indicated that the increased body weight could be attributed to greater amounts of fat [158]. In light of its apparent pro-adipogenic effects in rodents, Hao et al., went on to show that DES induced adipogenic differentiation of 3T3-L1 cells at both 1 and $10 \mu \mathrm{M}$. This could be inhibited by co-applying the ER antagonist ICI 182,780, suggesting that the effect is an estrogenic response [159]. The authors also found that DES administered at $10 \mu \mathrm{g} / \mathrm{kg}$ via maternal gavage from G12-P7 lead to increased body weight, adiposity, liver weight, and blood glucose concentrations in female C57BL/6J mice [159]. The effect was notably absent in male mice, and impossible to detect in higher dose groups $(25-100 \mu \mathrm{g} / \mathrm{kg})$ owing to high rates of fetal or neonatal death [159].

Compared to BPA, DES is similar in chemical structure, though a great deal more 
potent. Moreover, its range of receptor affinities and biological activities seems to be narrower and more uniformly estrogenic in character. Because of this, DES has received interest not only as a xenoestrogen in its own right, but also as a positive control in studies of other putative xenoestrogens [150]. The extent to which the effects of DES and the test compound overlap, so the logic goes, hints at the extent to which the test compound's effects are due to estrogenic responses. In order for this approach to hold, the negative control must be free of confounding sources of estrogenic stimulation, or at the very least every effort should be made to ensure that these confounds are held constant across treatment groups [160].

(a) Bisphenol-A (BPA)

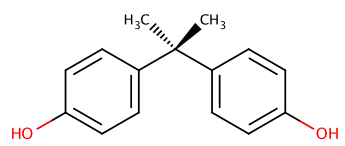

(b) Diethylstilbestrol (DES)

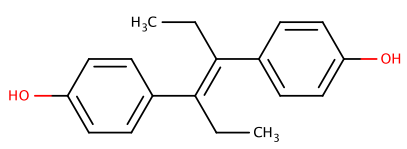

(c) $17 \beta$-estradiol

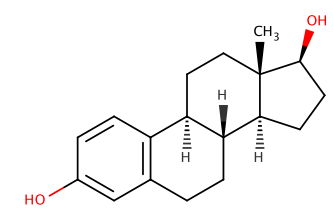

\subsubsection{Avoiding Litter Effects}

An additional concern raised by the NTP and other researchers in this field is the risk of over-estimation of effect sizes due to pseudoreplication errors [148]. Also called litter effects, these occur when siblings from the same litter are treated as independent statistical units. It is generally recognized that siblings from the same litter will show a high degree of similarity to each other simply due to a common genetic heritage and prenatal environment. The effects of the maternal manipulation thus become entirely confounded with the similarity inherent in siblings [161;162;163; 164]. One could either use just one randomly selected pup from each litter for later study, or average data across the entire litter and use that figure for statistical analysis [161;162; 163; 164]. The former is manageable, but wasteful in terms of animal life. The latter requires a massive number of extra animals, and the returns on this investment may be quite minor in proportion to the increase in reagents, space, and hands-on time required to carry out such an experiment. A solution that strikes a good balance is to treat the net quantity of pups produced at the outset as a population from which samples can be picked to produce a randomized complete block design. With this approach it is possible to utilize almost every pup from each litter, provided a sufficient number of timepoints or post-natal manipulations can be devised. By including one pup per litter in each statistical block, the issue of statistical pseudoreplication is obviated and litter effects become an asset, as it becomes possible to make between-treatment, within- 
litter comparisons. Since sex can be treated as a blocking factor as well, this approach allows differently sexed animals from the same litter to be fairly compared. This is the approach that we have chosen to use in this series of studies. 


\section{Chapter 2}

\section{The Neurobiology of Obesity}

In general, the aim of the projects described in this thesis is to understand how the obesogenic effects of BPA may be brought about through alterations to the developing neural feeding circuitry. Having only the end phenotype of hyperphagic obesity to work with in this case, it is reasonable to begin this investigation by drawing analogies to other experimental models wherein an obesity-prone phenotype is brought about through environmental manipulations early in life. The various manipulations, though different in certain ways, do appear to have a fairly high level of functional overlap with respect to their actions on the brain, and the hypothalamic feeding circuitry could be described as their final common pathway in the central nervous system. The systems targeted by BPA are no doubt different at the outset, but its actions on the final common pathway may yet be the same, and thus an understanding of the hypothalamic regulation of feeding is an essential basis for our research.

\subsection{HYPOTHALAMIC REGULATION OF ENERGY BALANCE}

The homeostatic maintenance and defence of energy balance is critical for the survival of the organism. While there can be substantial variation in either of calorie intake or energy expenditure in the day to day life of an organism, the two must balance each other in order to avoid starvation or obesity. For this reason, energy homeostasis is tightly regulated by the central nervous system, which as a whole integrates nearly all of the behavioural, physiological, and hormonal processes involved in the procurement, consumption, and utilization of energy. Fully predictable access to sources of energy can never be guaranteed nor, for that matter, can energy demands be reliably forecasted, at least in the context of our evolutionary history. The best compromise therefore is to make the fullest possible use of all available humoral and sensory cues to regulate energy balance in the short term, adjusting intake and expenditure to maintain something approaching neutrality. The breakdown of this process can lead to starvation, or much more commonly obesity and its attendant metabolic comorbidities such 
as type II diabetes, hypertension, cardiovascular disease, and more.

\subsection{THE HYPOTHALAMIC MELANOCORTIN SYSTEM}

Though the ventral hypothalamus has been implicated in the control of feeding and metabolism since at least the 1940s [165], the arcuate nucleus (ARC) has come into particular focus in the decades following the discovery of leptin. The melanocortin system comprises three populations of neurons, two of which are located in the ARC. These are the neurons that express proopiomelanocortin (POMC) and those that coexpress neuropeptide Y (NPY) and agouti-related peptide (AgRP). The third population inhabits the paraventricular nucleus (PVN) and is characterized by the expression of the melanocortin-4 receptor (MC4R). Early studies of these three populations first attested to their individual roles in the control of feeding. Central injection of AgRP [166] or NPY [167] potently stimulates feeding, while injection of the anorectic cleavage product of the POMC propeptide $\alpha$-melanocyte stimulating hormone $(\alpha-\mathrm{MSH})$ or the MC4R agonist melanotan-II inhibits feeding [168].

Leptin is a $16 \mathrm{kD}$ peptide hormone produced primarily in the adipose tissue, and secreted in quantities roughly proportional to the amount of stored fat $[169 ; 170]$. The leptin receptor is a member of the cytokine receptor family encoded by a single gene that gives rise to six splice variants (ObRa-f), of which only ObRb possesses the cytoplasmic domain needed for intracellular signalling [171]. Leptin binding to the ObRb receptor activates the Janus kinase (JAK)/Signal transducer and activator of transcription 3 (STAT3) pathway, leading to the phosphorylation and nuclear translocation of STAT3 [172; 173; 174]. Activation of this pathway leads to many transcriptional changes, including increases in ARC POMC and Suppressor of cytokine signalling 3 (SOCS3) expression, respectively effectors and negative feedback regulators of leptin signalling $[175 ; 176 ; 172]$. In both developmental and adult-onset models of obesity leptin continues to circulate in proportion to fat stores, yet apparently loses its inhibitory control over feeding. While the expression of the leptin receptor itself does not change, the downstream signalling pathways that it controls become less responsive to leptin and therefore exert only minimal effects on behaviour and physiology. Central leptin insensitivity, mediated in part by excessive transcription of SOCS3, is taken to be the main reason for the gradual dropping-off of leptin's anorectic effects under obesity $[177 ; 178]$. This reality has confounded any hope of combatting obesity through the use of supplemental exogenous leptin.

The behavioural and physiological changes associated with peptide upregulation are normally accompanied by delays associated with the needed transcriptional and translational processes. However, these neurons are also capable of more rapid action mediated by changes in their electrical activity. In an early study on the matter, Elias et al., showed that within two hours of injection, peripheral leptin induced SOCS3 
expression in both POMC and NPY/AgRP neurons, but it induced Fos expression only in POMC neurons [179]. Leptin thus elicited a response in both populations of neurons, but it only appeared to increase neural activity in those that expressed POMC. This was subsequently confirmed electrophysiologically, when it was shown that POMC neurons exhibit increased rates of action potentials when exposed to leptin [180]. This is mediated in part by ion channels on the POMC neurons themselves [180], but another portion of the effect involves changes in the cells that influence POMC neurons. The NPY/AgRP neurons form GABAergic synapses on the nearby POMC neurons, and because of the inhibitory influence of leptin on the NPY/AgRP neurons, POMC neurons are in turn disinhibited [180]. In $o b / o b$ mice that congenitally lack leptin, POMC neurons are under a state of constant inhibition relative to what is seen in wild-type animals, and this can be reversed by the administration of leptin [181], as well as by estradiol [182]. The fact that the default state of this circuit promotes tonic inhibition of the POMC neurons may account for why, of all the possible disturbances to energy homeostasis, obesity is the most common outcome [183].

The PVN is one of the main recipients of projections arising from neuropeptidergic cells in the ARC. Respectively anorexigenic and orexigenic fibers from POMC and AgRP/NPY neurons innervate the various neurosecretory and pre-autonomic neurons of the PVN to bring about behavioural and homeostatic changes associated with the hormonal status sensed by the ARC $[184 ; 185 ; 186]$. MC4R is expressed throughout the PVN, and appears to be the primary downstream receptor of ARC signals. The activity of MC4R neurons results from the balance of agonistic and antagonistic influences upon the MC4R receptor. The endogenous agonist for MC4R is $\alpha$-MSH, while $\mathrm{AgRP}$ is its endogenous antagonist [187].

\subsection{DEVELOPMENTAL MODELS OF OBESITY}

Manipulations to the early-life nutritional environment exert a reliable effect on the adult metabolic phenotype. Exposing pregnant animals to food restriction during gestation leads to offspring that demonstrate relative hyperphagia - a condition exacerbated by postnatal exposure to hypercaloric food [188;189; 190]. This is accompanied by a reconfiguration of hypothalamic peptide expression that supports a hyperphagic phenotype, including reduced POMC expression [191], and increased NPY expression in the ARC upon postnatal exposure to a high-fat diet [192]. Leptin signalling shows reliable alterations across the various implementations of the prenatal undernourishment paradigm. Rats exposed to gestational food restriction show increased leptin receptor expression at P1 as compared to normally reared controls, but decreased expression at three weeks of age [189]. A similar picture emerges in mice, with mice exposed to gestational food restriction showing increased ObRb expression during the early postnatal period [190; 193]. E12 fetuses of protein restricted dams show a similar increase 
in brain ObRb expression [194]. At P1, prenatally undernourished rats show reduced pSTAT3 expression in response to leptin, suggesting a deficit in leptin signalling in spite of the increased receptor expression [189]. Reduced anorectic responses to leptin persist into adulthood among prenatally food restricted rats, implying that this model leads to permanent organizational changes in feeding circuitry [195].

On the other hand, rats prenatally exposed to a high fat diet show increased body weight and hyperleptinemia in adulthood [196]. Similar to what is seen with prenatal undernourishment, this phenotype is exacerbated by postnatal exposure to a high-fat diet [196]. Offspring of obese dams exhibit an impaired anorectic response to leptin in adulthood, but the genesis of this phenotype is difficult to pinpoint, as offspring are exposed to maternal obesity through the gestational and lactational periods [197]. This dilemma can be solved by cross-fostering pups from overweight dams to control dams, and in such experiments leptin signalling remains impaired, implying a prenatal origin [198]. P1 offspring of obese dams had decreased NPY, POMC, and ObRb expression in the ARC, as well as decreased expression of the intracellular mediators of leptin signalling STAT3 and SOCS3 [199]. It is possible that these effects are harbingers of future leptin resistance and obesity. Over-nutrition confined strictly to postnatal life also adversely affects adult metabolism, leading to leptin resistance and a predisposition to develop obesity when weaned onto a high-fat diet [200; 201; 197]. These effects may be due to aberrant leptin signalling during the early postnatal period [197], the result of which may lead to permanent morphological abnormalities in leptin-sensitive neural circuits [185; 202; 203; 204].

Since the discovery of leptin and its characterization as a hormone involved in the regulation of feeding in adult animals, a second line of research has developed in parallel showing that leptin is also a very important signal during development. Under ordinary conditions, neonatal mice begin life with infinitesimal quantities of adipose tissue, and correspondingly minuscule levels of circulating leptin. Circulating leptin concentrations exhibit a surge during the first weeks of life that peaks between the $8^{\text {th }}$ and $10^{\text {th }}$ days of postnatal life $[185 ; 205]$. This leptin surge does not appear to influence food intake (nor does leptin in general do so until weaning), but it is critical for the development of leptin-sensitive neural circuitry [185]. Maternal under-nutrition from mid-gestation to weaning leads to large decreases in post-natal leptin secretion, and the complete absence of a leptin surge [206]. Maternal under-nutrition that is confined to the gestational period leads to an exaggerated leptin surge in pups, followed by a period of hypoleptinemia in the period that intervenes prior to weaning [207]. Pups of obese dams show an exaggerated leptin surge, and higher levels of circulating leptin throughout the second half of the 21 day pre-weaning postnatal period [197]. Given that all of these models are known to produce obesity and/or metabolic abnormalities in adulthood, the prevailing message appears to be that postnatal leptin levels must be kept within a certain range and that deviations in either the timing or quantity of 
postnatal leptin may adversely affect development.

\subsection{ESTROGENIC REGULATION OF FEEDING AND METABOLISM}

The sex steroids, most notably those in the estrogen family, play an important role in the control of feeding and metabolism. These hormones have both activational effects on feeding in the adult animal, as well as organizational effects on the feeding circuitry in the developing animal. Because BPA is a compound with noted estrogenic properties (see Section 1.3.1), it is important to explore the normal role of estrogen in the processes of feeding and metabolism, since many aspects of these could be affected by BPA exposure.

\subsubsection{Activational Effects of Estradiol on Feeding}

Other factors being equal, male rodents are easier to study because they possess a relatively stable hormonal profile, with only limited day-to-day variation. Female reproduction, in contrast, is under the control of an estrous cycle with a period of 4-5 days. This cycle begins with the onset of reproductive maturity and continues until reproductive senescence, unless interrupted by pregnancy, lactation, pseudopregnancy, or any number of stressors. The cycle can be broken into four separate stages: diestrus I (also known as metestrus), diestrus II, proestrus, and estrus. Circulating estradiol concentrations begin to rise mid-way through diestrus II, peaking during the afternoon of proestrus. There is a marked spike in Luteinizing Hormone (LH) on the eve of estrous, and this event triggers ovulation later in the night. Primed by estrogen, progesterone levels rise sharply subsequent to ovulation, facilitating sexual receptivity. This period is called behavioural estrus in order to distinguish it from vaginal estrus. Circulating progesterone increases in a more prolonged fashion during diestrous I, but in this case it tends to inhibit sexual receptivity, an effect consistent with the hormone's role in maintaining pregnancy. Because of the cyclic variations in the ovarian hormones, as well as the gonadotrophic hormones that drive them, day-to-day variability is an unavoidable feature of studies involving females. Aside from variations in behaviours directly concerned with reproduction, a number of other regulatory behaviours also show cyclic variation in female rodents. Of particular interest here are cyclic variations in feeding behaviour.

The effects of estradiol on food intake are well characterized in rodent models, where the food intake of cycling animals shows a transient decrease during estrus [208; 209; 210; 211; 212; 213]. Rats and mice eat sparingly during the day, preferring instead to consume the majority of their food at night. Since vaginal smears are usually collected by researchers during the day, it is most accurate to say that the drop in food intake occurs on the night that marks the transition between proestrus and es- 
trus ${ }^{1}$. When feeding behaviour is analyzed at the microscopic level, that is, at the level of individual meals rather than cumulative food consumption, the periovulatory reduction in food intake can be attributed to smaller meal sizes with an increase in meal frequency that does not quite compensate for the overall reduction in caloric intake [209].

Ovariectomized rats cease to show cyclic variations in behaviour, vaginal cytology, and as might be expected, feeding as well ${ }^{2}$. These animals begin gaining weight, and in short order become obese [216]. This rapid weight gain occurs in part because postoperative animals are hyperphagic, with food intake that may be up to $50 \%$ greater than what would have been seen during diestrus pre-operatively [209; 210; 217]. The greatest relative gains in food intake are seen during the light phase of the $24 \mathrm{~h}$ cycle, and these gains derive from larger (but not more frequent) meals [218]. While the effects of ovariectomy on cumulative food intake are transient and begin to disappear after a few weeks, the change in body weight remains [209]. Plasma estradiol levels are virtually nil following the ovariectomy procedure, and this state of affairs is unlike any stage of the estrous cycle, where estradiol is always present at detectable levels. Since food intake in newly ovariectomized females is markedly higher than it had been at any point in preoperative life, Drewett suggested that estradiol has both tonic and phasic influences on feeding [210]. The behavioural and metabolic response to ovariectomy can be rescued by a regime of physiological estradiol treatment ( $2 \mu \mathrm{g}$ every fourth day), mimicking the naturally occurring cyclic variations in plasma estradiol [219; 220]. Progesterone, another important ovarian hormone, is also elevated during the periovulatory period. These circumstances would seem to implicate it as well in the cyclic control of feeding, but studies of progesterone treatment in ovariectomized rats have not borne this out. In fact, progesterone co-treatment dose-dependently inhibits the body weight and food intake reducing effects of estradiol treatment [217], though it should be noted that the doses of progesterone used in this study were pharmacological in proportion and this effect has not been universally replicated [218]. Still, the tendency for progesterone to counteract the anorectic effects of estradiol may explain why combined contraceptive therapy in women does not lead to changes in appetite or body weight [221].

When dealing with the study of energy balance in general, it may be possible to set aside the issues of thermoregulation and locomotor activity and focus instead on whole-body energy expenditure, something that is closely related to the other measures, but at the same time somewhat more fundamental. This approach neglects the important question of just how the animal is spending its energy, but within the scope of this thesis that may be an acceptable compromise. Following ovariectomy, the an-

\footnotetext{
${ }^{1}$ This drop is sometimes reported as belonging to the proestrus stage (e.g. [209]), but more commonly it is considered to belong to the estrus stage (e.g. [210]), and this is the scheme we have adopted.

${ }^{2}$ Males, on the other hand, show no cyclic variations in feeding, and actually exhibit a slight decrease in food intake when gonadectomized that can be rescued by testosterone replacement [214; 215].
} 
imal's rate of energy expenditure falls well below what would normally be seen, even during the diestrus nadir [222]. In the early 1980s, Laudenslager et al., first demonstrated that ovariectomized rats receiving chronic estradiol supplementation had, as compared to vehicle-treated rats, elevated rates of $\mathrm{O}_{2}$ consumption and significantly less weight gain following the ovariectomy operation [223]. More elaborate studies became possible with the advent of affordable solid-state indirect calorimetry equipment and these have confirmed early suggestions that estradiol, acting either cyclically in intact animals [224; 225], or exogenously in ovariectomized animals [226; 227] increases energy expenditure and shifts substrate utilization toward fatty acids. These effects likely depend on the central nervous system, for recent studies have shown that central administration of estradiol increases the thermogenic activity of the brown adipose tissue, an organ concerned almost entirely with scrubbing off surplus energy [226]. Estradiol remains anorectic, but does not alter energy expenditure in intact animals [227]. It may be, then, that exogenous estradiol can at best make up for the shortfall in energy expenditure seen following ovariectomy, without having any additive effects in intact animals over and above the tonic influence of endogenous estradiol.

The cumulative effect of the aforementioned early studies was a demonstration of the activational effects of estrogens on feeding and metabolism. From the outset, the classical estrogen receptors were seen as likely candidates for mediating the anorectic effects of estradiol, and this was confirmed when it was noted that both male and female mice lacking ER $\alpha$ showed increased adiposity and impaired glucose tolerance beginning in early adolescence [228]. Curiously, these animals do not show increased food intake when compared to wild-type controls, indicating that congenital lack of $\mathrm{ER} \alpha$ increases feed efficiency without increasing food intake itself [228; 229]. ER $\alpha$ knockout females do not respond to the anorectic effects of exogenous estradiol, and they are not greatly affected by ovariectomy [229]. A similar phenotype is seen in aromatase-deficient mice that are congenitally incapable of completing the final step of estrogen biosynthesis [230;231]. In both cases there is a marked departure from the phenotype generated by adult ovariectomy. Aside from the fact that weight gain in estrogen-deficient ovariectomized females is due not only to increased feed efficiency but also to patent hyperphagia, there is the also the issue of males, who produce only vanishingly small quantities of endogenous estrogens, and, of course, cannot be ovariectomized at all, yet respond similarly to females to ER $\alpha$ knockout. Discrepancies such as these are currently unexplained, but it is likely that the absence of some or all of estradiol's organizational effects plays a major role.

The anorectic effect of estradiol was determined to be due to centrally located estrogen receptors by way of an elegant experiment comparing the relative potency of centrally and peripherally administered ICI-182,780, a pure ER antagonist that is incapable of crossing the blood-brain-barrier. Since ICI-182,780 was only capable of exerting an effect on feeding when administered centrally, it follows that the anorec- 
tic effects of endogenous estradiol likely depend on its access to the central nervous system [232]. Both ER $\alpha$ and ER $\beta$ can be found in feeding-relevant hypothalamic nuclei, though it is to ER $\alpha$ that the majority of estrogen's anorectic effects are attributed [213]. Recent investigations have taken to studying the role of estrogenic signalling at the level of individual hypothalmic nuclei. Adult knockdown of ER $\alpha$ specifically in the VMH leads to an obese, hyperphagic, and hyperglycemic phenotype that differs from congenital ER $\alpha$ mutants primarily in the presence of hyperphagia [233]. Mice constitutively lacking ER $\alpha$ in the ventromedial hypothalamus (VMH) by way of cre-mediated recombination in Steroidogenic factor 1 (Sf1) neurons show an obesity-prone phenotype [234]. These animals also showed reduced energy expenditure and brown adipose tissue thermogenesis [234], suggesting that the VMH is an important site mediating the effect of estradiol on sympathetic nervous system outflow. On the other hand, knockdown of ER $\alpha$ in POMC neurons in the nearby ARC leads to increased body weight in females, apparently driven by increased lean mass [234]. These animals are relatively hyperphagic and respond poorly to exogenous leptin, though paradoxically possess greater than average levels of energy expenditure [234].

Ovariectomized mice lacking the leptin receptor specifically in POMC neurons gain relatively more weight than ovariectomized mice that are intact in that regard [235], suggesting that the control exerted on feeding by leptin and the estrogens has some degree of overlap within the melanocortin system. A subset of anorexigenic POMC neurons in the ARC express $\mathrm{ER} \alpha, 30 \%$ is the figure usually given [236;237; 182]. The proportion of POMC neurons that express $\mathrm{ER} \beta$ is vanishingly small by comparison [236], a finding that is consistent with the apparent lack of a metabolic phenotype in $\operatorname{ER} \beta$ knockout mice [213]. POMC expression in the ARC is higher in intact females than males [238], and exogenous estradiol is capable of increasing POMC expression and $\alpha$-MSH concentration in ovariectomized females [239; 240]. In this capacity, estradiol could be considered as somewhat analogous to other metabolic hormones like leptin, which also control the expression of hypothalamic feeding peptides. This similarity was further explored by Gao et al., who found that estradiol treatment sufficient to reduce energy intake results in activation of POMC neurons, as evidenced both by immediate early gene expression and neuronal excitability [182]. These authors also found that estradiol induces STAT3 phosphorylation at the tyrosine 705 residue, and this could be interpreted as mimicking the action of leptin [182]. AgRP/NPY neurons are also involved in the estrogenic control of feeding, showing complementary responses to estradiol treatment, including a reduction in the transcription of those genes, and attenuated fasting-induced cFos expression [241]. Interestingly, there are apparently no AgRP/NPY neurons that co-express ER $\alpha$ [241]. This may be because AgRP/NPY neurons are second-order recipients of estrogenic signalling, or perhaps because membrane-bound estrogen receptors are more important here. Kwon et al., recently found that intracerebroventricular administration of a GPR30 antagonist blocks 
exogenous estradiol's ability to induce hypothalamic pSTAT3 and reduce food intake [242], and these findings are likely to soon be joined by others pointing to the importance of membrane estrogen receptors in the control of feeding and metabolism.

While the activity of estradiol itself may be sufficient to explain its effects on feeding, its interaction with other feeding-relevant hormones cannot be ignored. Leptin, already known to mingle with estrogen-sensitive neurons in the hypothalamus, plays an important role in both feeding and the control of reproduction. As might be expected, certain aspects of the leptin system show cyclic variability in females. Expression of the short form of the leptin receptor in the ARC, VMH, and choroid plexus is lowest on the day of proestrus, suggesting cyclic alterations in the permeability of the brain to leptin [243]. Moreover, the long form of the leptin receptor (ObRb), present in the ARC and VMH is also down-regulated during proestrus [243]. In contrast, leptin production and secretion in intact female rats is increased during proestrus relative to diestrus [244]. While estradiol is capable of improving leptin sensitivity in diet-induced obese female mice [227], it is apparently not necessary for leptin sensitivity in the first place. Leptin levels rise in the weeks following ovariectomy - an expected consequence of the attendant obesity — but both neurobiological and behavioural measures of leptin sensitivity remain intact [245].

\subsubsection{Organizational Effects of Sex Steroids on Energy Balance}

The processes of feeding and metabolism differ between the sexes in a number of respects. Compared to males, female rodents tend to resist diet-induced obesity [246], at least so long as they retain ovarian function. There are more subtle differences, too, for example female rodents show greater rebound feeding on the morning following an overnight fast [247]. Many more differences exist beyond these and those discussed in Section 2.4.1, and while the link between these differences and female-typical levels of circulating estradiol is apparent in most cases, it is clear that they alone cannot explain everything. Sex differences in general come down to more than just the levels of sex hormones in circulation at any given moment, and so it is likely that in the specific cases of feeding and metabolism, females differ from males by more than just their estradiol concentrations. The early-life hormonal environment has a long-lasting impact on development, programming reproductive and homeostatic systems in a sex-specific manner. Acting in this capacity, hormones are said to exert organizational effects, and these may consist of changes in morphology or physiology, in the response to hormones later in life, and in the presence or absence of certain sex-specific behaviours.

Once again, early studies into the matter established an empirical basis that has only begun to be elaborated upon in recent years. Early post-natal masculinization of female rat pups, achieved by injecting testosterone during the first week of post-natal life, predisposes female rats to increased body weight in adulthood [248; 249]. Since 
ovarian function and estrous cyclicity are both disrupted in these animals, two hypotheses emerged from these findings. According to the first: it may be that testosteronetreated females become overweight because, lacking the appropriate secretion of estrogen, they mimic ovariectomized animals in adulthood. Alternately, it may be that testosterone exerts an organizational influence on the systems that regulate feeding and metabolism, and these organizational changes are at the root of the resulting phenotype. When the phenomenon was first discovered, too little was known of these systems to know for certain. Still, clever experimental design allowed certain inferences to be made. Beatty et al., concluded that neither the level of circulating estradiol, nor the body's sensitivity to it could reasonably account for weight gain in masculinized females. Though they are heavier to begin with, both control and masculinized females show a similar trajectory of weight gain following ovariectomy, and they also show a similar relative anorectic response to exogenous estradiol [249].

The study of organizational influences of sex steroids on feeding circuitry has recently been revisited in light of modern developments in the neurobiology of feeding. In 2011, Nohara et al., found that relative to control females, neonatally masculinized females had impairments in their responses to leptin [238]. In these animals leptin failed to stimulate a reduction in feeding or an increase in POMC expression. This was apparently not due to an alteration in leptin-induced pSTAT3 signalling, as might be expected if one uses conventional models of leptin resistance as a basis for comparison [238]. The initial phases of this study were carried out by injecting female mice with $100 \mu \mathrm{g}$ testosterone on P1, but this approach on its own cannot be conclusive. Testosterone is a pro-hormone that can be converted into either of two forms: the potent androgen dihydrotestosterone by the enzyme $5-\alpha$ reductase, or one of three estrogens by aromatase. The full gamut of masculinization and defeminization responses to testosterone depend on a combination of these mechanisms. Nohara et al., found that for the most part, neonatal estradiol treatment could recapitulate the leptin resistance and obesity observed in animals treated with testosterone, implicating local aromatization in that process [238]. On the other hand, only dihydrotestosterone was able to match testosterone's ability to program reduced POMC expression, evidence that, when combined with the foregoing, suggests processes of both masculinization and defeminization are at work in the development of energy balance circuitry. Some questions remain, however. For instance, neonatal testosterone and estradiol treatment both induce programmed leptin resistance in females, but since males are not generally leptin resistant by nature, it is difficult to describe this outcome as 'masculinization'. Males, to be sure, are more prone to diet-induced obesity and its attendant metabolic sequelae, but programmed leptin resistance is not involved in this. Recently, the authors reported that neonatal testosterone treatment programs leptin resistance in males as well [250], and this too is difficult to square with 'masculinization'. Nevertheless, these studies do demonstrate that the developing hypothalamus is sensitive 
to the effects of both androgens and estrogens, and that alterations in the hormonal environment can be at least as effective as alterations in the nutritional environment in triggering long-lasting changes in energy homeostasis.

\subsection{EPIGENETIC IMPRINTING}

While the organizational effects of the early-life hormonal state have been known to comprise changes of anatomical and physiological character - the relative growth or retreat of various specialized organs and brain regions - a new set of mechanisms collectively referred to as epigenetic imprints has recently aroused much interest. The term epigenetic imprinting does not describe a single molecular process, rather it describes a collection of processes that have in common the ability to modulate gene expression in a manner that is permanent or at least very long-lived, and in a manner that possesses a level of specificity such that it can be confined to a particular subset of cells residing within a nucleus in the brain. These imprints may consist of changes related to chromatin remodelling - typically achieved by post-translational modification of the histone proteins around which DNA is wound - or by the attachment of methyl groups to cytosine residues in the DNA itself, often in the context of CpG dinucleotide islands in the promoter regions of select genes.

\subsubsection{Epigenetics in Developmental Models of Obesity}

Developmental models of obesity are known to lead to changes in hypothalamic gene expression, or at least changes in the reactivity thereof. This suggests the existence of epigenetic imprints that maintain these differences beyond the perinatal period during which they are presumably arranged. Numerous lines of emerging evidence suggest that this is indeed the case, and that the genes coding for the hypothalamic energy balance peptides are subject to epigenetic imprinting via DNA methylation. The earliest study to explore this was carried out by Plagemann and colleagues using a model of postnatal over-nutrition. While the authors found no difference in DNA methylation of the NPY promoter between treatments, they did observe several instances of increased methylation in the POMC promoter in animals subjected to postnatal overnutrition [251]. These animals were hyperleptinemic in adulthood, yet showed levels of POMC mRNA and protein expression that were comparable to control animals. This state of putative leptin resistance can be explained in part by POMC hypermethylation, because regressing the rate of $\mathrm{CpG}$ methylation against a value representing arbitrary units of POMC expression per unit of serum leptin yields a significant negative correlation. In other words, the greater the rate of $\mathrm{CpG}$ methylation upstream of POMC, the less effective leptin is at increasing POMC expression [251]. Marco et al., found similar results, albeit with a model of postnatal (PND21-90) high-fat diet exposure. In this 
study, obese animals showed the hallmarks of impaired leptin signalling, namely a failure to up-regulate POMC expression in the face of obesity and hyperleptinemia, and this was correlated with CPG hypermethylation of the POMC promoter [252]. The opposite scenario of perinatal growth restriction has produced somewhat less clearcut results. In one study, pups subjected to intrauterine growth restriction were found to have decreased methylation of the POMC promoter region in the ARC, though this was paradoxically associated with lower levels of POMC mRNA expression in the same tissue [253]. This effect was not replicated by Shin et al., who found no difference in POMC promoter methylation in response to combined intrauterine and postnatal growth restriction, though their model did produce a reduction in POMC mRNA expression [193].

If it is true that early-life over-nutrition generally results in a state of hyperleptinemia during the period of the manipulation, and that early-life under-nutrition does the opposite, one might hypothesize a positive correlation between leptin levels and subsequent POMC promoter methylation. A study by Palou et al., addressed this possibility by raising rat pups under ordinary conditions with or without daily oral leptin treatment (calculated to be roughly $5 x$ that normally received in the milk, from P1-21). Pups were subsequently exposed to a high-fat diet in adulthood, and it was found that leptin treated animals possessed a degree of resistance to the obesogenic effects of that diet. Interestingly, the authors found an interaction between early-life leptin treatment and postnatal diet, such that leptin treated animals tended to exhibit POMC promoter hypermethylation under a normal diet, yet the situation was completely reversed by the high-fat diet with control and leptin treated animals respectively showing POMC hyperand hypomethylation [254]. This effect was especially prominent at the Sp1 binding site -166bp upstream of the transcription start site [254]. While these results attest to a complicated regulatory picture, they demonstrate that the postnatal hormonal state can affect epigenetic imprinting independent of the contemporaneous nutritional state, and also that there remains substantial plasticity within this system such that it can be affected by adult diet.

Direct manipulation of the transcriptional and epigenetic machinery within POMC neurons produces an exaggerated form of the obese and hypermethylated POMC promoter syndrome observed in the aforementioned models of obesity. Deletion of the gene coding for methyl-CpG-binding protein 2 (MeCP2) specifically in POMC neurons leads to adult onset obesity, coupled with reduced POMC mRNA expression and widespread hypermethylation of its promoter region [255]. Both the epigenetic and expressional changes appeared to level out over the lifespan, and thus by 15 months of age there were no differences in methylation between mutant and wild-type mice [255].

The studies of Plagemenn et al., Marco et al., and Palou et al., have focused on the stretch of DNA lying between 406 [251] to 540bp [252] upstream of the POMC tran- 
scription start site. It is within this stretch that one finds the binding site for Specificity protein $1(\mathrm{Sp} 1)$, a protein that is capable of forming a complex with pSTAT3 and activating POMC gene expression [256]. Interestingly, changes in $\mathrm{CpG}$ methylation in the neighbourhood of the Sp1 binding site are one of the most common observations in studies of this sort [251;252]. It has been known for some time that this region is sufficient to drive POMC expression in experimental systems, though this promoter region on its own is specific only to POMC expression in the anterior pituitary [257]. This is an important consideration, because the regulation of POMC expression can differ substantially between the anterior pituitary and ARC [258]. Hypothalamic-specific POMC expression requires a region between -9 and $-13 \mathrm{~kb}$ from the POMC transcription start site [259]. This stretch contains in both humans and mice two regions with a relatively high level of conservation called nPE1 and nPE2. Either of the two on its own is sufficient to drive approximately normal neural expression of POMC, but the absence of both results in its complete ablation [259]. Interestingly, nPE2 possesses a conserved element that is capable of binding to a variety of nuclear receptor transcription factors including ER $\alpha$, which due to its frequent co-expression with POMC is the most plausible target for this element in-vivo.

\subsubsection{Epigenetics in Sexual Differentiation and Endocrine Disruption}

The role of epigenetic imprints in the formation and maintenance of aberrant metabolic phenotypes induced by the early-life nutritional states is thus becoming an accepted mechanism. Meanwhile, there is a great deal of evidence that similar processes are invoked during the process of sexual differentiation, which happens as a normal consequence of sex-specific variations in the hormonal milieu [260]. The nature of these epigenetic imprints changes over the developmental timespan, presumably as a consequence of changes in the hormonal environment. For example, methylation of the $\mathrm{ER} \alpha$ promoter in the Pre-optic Area (POA) is on the average lower in female rats than in males, and this difference can be eliminated by perinatal masculinization with estradiol benzoate or with simulated maternal grooming [261]. However, the converse has also been observed, with Schwarz et al., showing significantly more DNA methylation in the ER $\alpha$ promoter in female rats [262]. Since these two studies examined different promoter regions of the $\mathrm{ER} \alpha$ gene, the complexity and functional ambiguity of DNA methylation is the message that resounds here.

There is also emerging evidence that early-life exposure to endocrine disrupting compounds can result in epigenetic imprints, and these may be the basis for the longlasting effects that are caused by exposure to these substances [263]. Early studies into this possibility were carried out using the agouti viable yellow $\left(\mathrm{A}^{\mathrm{vy}}\right)$ mouse, which possesses a mutant promoter on the Agouti gene that results in widespread ectopic Agouti expression and a characteristic pattern of coloration. Given that the animals in 
question are, for all practical purposes, genetically identical, differential methylation is invariably brought about through the action of some kind of environmental stimulus [264]. Given that the methylation status of the Agouti gene is demonstrably plastic, and that status thereof can be readily inferred simply by observing the coat colour of affected mice, it has become a popular model for evaluating putative epigenetic effects of environmental stimuli. Early studies into the effect of BPA on this phenomenon suggested that gestational exposure to low-dose BPA could shift the coat colour of $\mathrm{A}^{\mathrm{vy}}$ toward the yellow end of the spectrum, thus indicating a decrease in methylation [265; 266]. Recently, Rosenfeld et al., attempted to replicate these findings, but only found a difference in the proportion of $\mathrm{A}^{\mathrm{vy}}$ offspring when dams were exposed to a high dose of BPA was combined with the phytoestrogen genistein $(50$ and $250 \mathrm{mg} / \mathrm{kg} / \mathrm{diet}$ respectively) [267]. This may have been due not to an epigenetic effect on the pups, but rather differential in-utero survival of $\mathrm{A}^{\mathrm{vy}}$ conceptuses, since the change in ratio was due to fewer a/a pups being born, not an increase in the number of $A^{\text {vy }}$ pups [267].

Studies using in-vitro exposure to BPA suggest that it is capable of regulating the activity of both maintenance and de novo DNA methyltransferases, down-regulating expression of the former and having mixed effects on the expression of the latter [268]. $\mathrm{BPA}$ also up-regulates the expression of the methylated CPG binding proteins Mecp2e1 and Mecp2e2 [268]. Gestational exposure to environmentally relevant doses of BPA leads to sex-specific, dose-dependent alterations in hypothalamic ER $\alpha$ mRNA expression in mice, and the direction of these changes is positively correlated expression of the de-novo DNA methyltransferase Dnmt1 [269]. As of yet, there is no evidence that either endo- or xenoestrogens can affect epigenetic imprinting of hypothalamic energy balance genes. Nevertheless, there is reason to suspect that such a relationship is possible, given that epigenetic plasticity is known to exist in those genes, and BPA is known to affect epigenetic imprinting.

\subsection{SUMMARY OF REMAINING QUESTIONS}

The aim of the foregoing review was to demonstrate that there is a good deal of evidence suggesting that BPA can affect the development of obesity, that it acts through a variety of estrogenic and non-estrogenic mechanisms, that human exposure can be modelled in a laboratory environment, and that studies having done so raise concerns about the safety of that compound. Conditions in the perinatal period can have a long-lasting impact on metabolism and body weight homeostasis, and exposure to either nutritional or hormonal challenge can predispose animals to obesity later in life. Given that the ARC and the energy balance circuitry it contains is the final common pathway of many developmental models of obesity, we hypothesize that exposure to BPA will predispose animals to obesity by altering the development of these circuits. 


\title{
Chapter 3
}

\section{Organizational Effects of Perinatal Exposure to Bisphenol-A And Diethylstilbestrol on Arcuate Nucleus Circuitry Controlling Food Intake and Energy Expenditure}

\author{
H. MacKay, Z.R. Patterson, R. Khazall, S. Patel, D. Tsirlin \& A. Abizaid \\ Endocrinology 154: 1465-1475, 2013 \\ Reprinted with permission of The Endocrine Society and HighWire Press (see Ap- \\ pendix B)
}

\subsection{INTRODUCTION}

Bisphenol-A (BPA) is plastic monomer and chemical plasticizer commonly used in the production of polycarbonate plastics and epoxy resins. Owing to its ubiquitous use in food and drink containers, low-level human exposure to BPA is nearly universal (see Section 1.1). BPA is commonly described as a xenoestrogen-an exogenous estrogenmimic - due to its ability to bind to and activate both nuclear and membrane-bound estrogen receptors (see Section 1.3.1). In addition to its classical estrogenic capacity, first noted in 1936 [51], BPA is also active at a number of other nuclear receptors, occasionally with even greater potency (see Sections 1.3.2, 1.3.3, 1.3.4). Because of its spectrum of biological activities, there is reason to suspect that BPA may exert an organizational influence on the development of neuroendocrine systems that use the aforementioned receptor systems as a currency.

Early risk-assessment studies identified $50 \mathrm{mg} / \mathrm{kg} /$ day as the lowest observed adverse effect level (LOAEL); the United States Environmental Protection Agency (EPA) computed its reference dose of $50 \mu \mathrm{g} / \mathrm{kg} /$ day using a safety margin of 1000-fold [270]. Nevertheless, it is becoming clear that BPA alters the function of a number of organ systems at levels well below this putatively harmless reference dose [271]. Of particular 
interest is the possibility that early-life BPA exposure may lead to obesity and metabolic dysfunction in adulthood. This hypothesis has been supported by a number of recent studies, all of which demonstrate obesogenic and diabetogenic effects in low-dose animal models (see Section 1.5).

In this series of experiments, we sought to develop a model of environmentally relevant oral BPA exposure during pregnancy and lactation and use it to study metabolic parameters in adult offspring of BPA and DES-treated dams. Here we hypothesized that early-life BPA exposure would lead to alterations in the adult organization of the hypothalamic melanocortin system, particularly in the homeostatic circuitry located in the arcuate nucleus (ARC). To test this, we raised CD-1 mice exposed either to one of two doses of BPA, DES, or a purified Control diet to adulthood, and examined their metabolic function, propensity to diet-induced obesity, glucose intolerance, and numerous neuromorphological markers.

\subsection{METHODS}

\subsubsection{Animals}

All procedures were approved by the Carleton University Animal Care Committee and followed the guidelines of the Canadian Council on Animal Care. This study used two separate cohorts of mice, with the first being used for the long-term, diet-induced obesity study and the second for the histology study. Virgin female CD-1 mice $(N=20$ for Cohort 1, $N=16$ for Cohort 2) were purchased from Charles River and housed in polypropylene cages with ad-lib access to purified AIN93G control diet (Research Diets) and tap water in glass bottles. Animals were maintained on a $12 \mathrm{~h} \mathrm{light/dark} \mathrm{cy-}$ cle (lights on at 8:00). Female mice were weight-matched and assigned into treatment groups prior to mating. These females were housed in pairs along with a male mouse. AIN93G was used as the control diet, as well as the basis for the three treatment diets. BPA or DES ( $>99 \%$ pure, Sigma Aldrich) was added to AIN93G by incorporation with the oil component of these diets (see Table A.1 for details on diet composition). Mated females were inspected every morning for evidence of a vaginal plug, the presence of which was taken to mark gestational day 0 (G0). Confirmed pregnant females were single-housed and placed immediately on one of four specially prepared diets: (1) AIN93G control diet (Control), (2) AIN93G with $1 \mu \mathrm{g} / \mathrm{kg}$ diet BPA (0.001 ppm BPA), (3) AIN93G with $20 \mu \mathrm{g} / \mathrm{kg}$ diet BPA (0.02 ppm BPA), (4) AIN93G with 4 $\mu \mathrm{g} / \mathrm{kg}$ diet diethylstilbestrol (0.004 ppm DES). DES is a well-characterized ER ligand, and was included as a positive control $[159 ; 150]$. Dams were maintained on these diets throughout pregnancy and nursing. Food intake and body weight of dams was recorded daily in order to estimate exposure to the experimental compounds. 
(a) Study design for Cohort 1

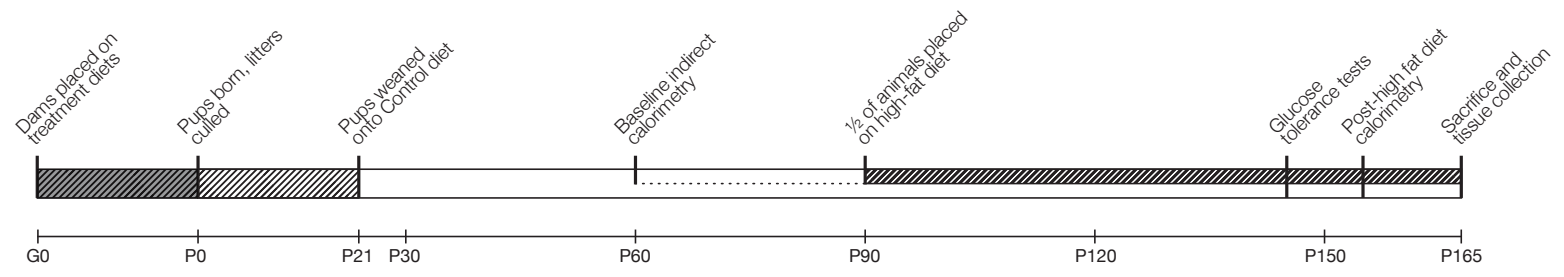

(b) Study design for Cohort 2

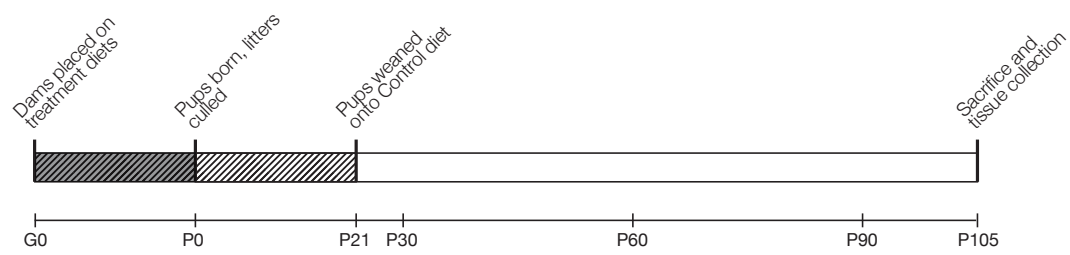

\subsubsection{Weaning \& Early life}

Litters were adjusted on P2 to have 10-12 pups/litter with equal sex ratios where possible. On P21, pups were weaned and housed in groups of 2-4 according to sex and litter and maintained on the Control diet. On P60, a subset comprising 2 males and 2 females from each litter was randomly selected to later be placed on the high fat diet; others remained on the Control diet for the duration of the study. At P90, all animals were single-housed ( $N=160$ for Cohort $1, N=128$ for Cohort 2$)$.

\subsubsection{Estrus Cycle Monitoring}

The estrus cycle was monitored by vaginal lavage in post-pubertal female offspring for at least eight days prior to any experimental manipulation or measurement. Experiments were generally carried out irrespective of estrous cycle, but proestrous animals will be removed from subsequent analysis because this phase of the estrous cycle is associated with altered feeding behaviour and neuropeptide expression [241]. 


\subsubsection{Diet-Induced Obesity}

At three months of age, a subset of animals from Cohort 1 was placed on a high-fat diet (HFD) $(60 \%$ of $\mathrm{kCals}$ from fat, $5.24 \mathrm{kCal} / \mathrm{g}$, Research Diets, see Table A.1) to explore their propensity to diet-induced obesity (DIO). Remaining animals continued on the AIN93G control diet $(15.8 \%$ of $\mathrm{kCals}$ from fat, $3.9 \mathrm{kCal} / \mathrm{g})$ throughout the experiment.

\subsubsection{Indirect Calorimetry}

Analysis of whole-body energy expenditure was performed on animals in Cohort 1 on approximately P60, and again at approximately P155 using an open-circuit indirect calorimetry system (LabMaster, TSE Systems). Measurements on P60 were gathered from the subset of animals destined to be placed on the high-fat diet, and measurements on P155 were gathered from all animals, thus yielding values pre- and postdiet-induced obesity in that subset, and contemporaneous data from Chow-fed animals from the other subset. Oxygen consumption $\left(\mathrm{VO}_{2}\right)$, carbon dioxide production $\left(\mathrm{VCO}_{2}\right)$, and locomotion were measured. Respiratory Exchange Ratio (RER) was calculated using the following equation:

$$
\mathrm{RER}=\frac{V C \mathrm{O}_{2}}{V \mathrm{O}_{2}}
$$

Energy expenditure was calculated using the modified Weir equation:

$$
k \text { cal } / h=\frac{3.941 * V O_{2}+1.106 * V C O_{2}}{1000}
$$

Animals were given 24 hours to habituate to the chambers, followed immediately by 24 hours of recording.

\subsubsection{Glucose Tolerance Tests}

Intraperitoneal Glucose Tolerance Tests (GTTs) were performed on animals in Cohort 1 after approximately 60 days of high-fat diet exposure. Mice were fasted overnight. At 9:00, a baseline blood glucose measurement was collected from the tail vein using a Bayer Contour handheld glucometer (Bayer Healthcare). Mice were injected with $20 \% \mathrm{D}$-glucose at $2 \mathrm{~g} / \mathrm{kg}$ body weight, and post-injection blood glucose readings were collected at 15, 30, 60, and 120 minutes following injection. Mice were excluded from analysis if they did not exhibit an increase of at least $1 \mathrm{mMol} / \mathrm{L} 15$ minutes postinjection. 


\subsubsection{Hormonal Analyses}

The concentrations of interleukin-6, insulin, leptin and resistin in plasma collected from Cohort 1 at the time of sacrificed were measured simultaneously using MilliPlex Mouse Adipokine kits (Millipore) and a Luminex 100 analyzer (Luminex) according to the provided directions. The intra-assay coefficients of variation for IL- 6 , insulin, leptin, and resistin were respectively $7.74 \%, 8.33 \%, 7.96 \%$, and $6.29 \%$. The inter-assay coefficients of variation for IL-6, insulin, leptin, and resistin were respectively $11.69 \%$, $9.38 \%, 8.19 \%$, and $10.84 \%$.

\subsubsection{Body Fat Determination}

Carcasses of animals from Cohort 1 were frozen at $-20^{\circ} \mathrm{C}$ prior to dissection. Perigonadal, retroperitoneal, subcutaneous fat pads, and interscapular brown adipose tissue (BAT) were dissected and weighed by an observer blind to treatment condition.

\subsubsection{Liver Histology}

Liver samples were taken from animals in Cohort 1 at the time of sacrifice and immersion fixed in $4 \%$ paraformaldehyde overnight at $4{ }^{\circ} \mathrm{C}$. Liver samples were subsequently cryoprotected in $30 \%$ sucrose and sectioned at $15 \mu \mathrm{m}$ on a cryostat (Thermo Scientific). Slices were thaw-mounted on gelatin subbed slides, and left to dry at room temperature for 60 minutes. For Oil Red $\mathrm{O}$ staining, slides were rinsed briefly in $\mathrm{dH}_{2} \mathrm{O}$ followed by $60 \%$ isopropanol. Slides were stained with freshly prepared, pre-heated $0.3 \%$ Oil Red O (Sigma) in $60 \%$ isopropanol at $60^{\circ} \mathrm{C}$ for 15 minutes. Following staining, slides were briefly rinsed in $\mathrm{dH}_{2} \mathrm{O}$, counterstained with Mayer's hematoxylin, and blued with tap water. Slides were coverslipped with glycerol and imaged immediately. For periodic acid-Schiff staining, slides were immersed in 1\% periodic acid for 10 minutes. After washing with $\mathrm{dH}_{2} \mathrm{O}$, slides were stained in Schiff reagent (Sigma) for 10 minutes, washed, and counterstained with Mayer's hematoxylin. After bluing with tap water, slides were dehydrated through an ethanol series, and coverslipped with Permount (Fisher Scientific). In both cases, images were captured using an Olympus BX51 microscope equipped with a colour camera (DVC-2000C, DVC Company). Images were analyzed using ImageJ (National Institutes of Health). In each image the area not occupied by any tissue, the area occupied by counterstained tissue (with negative Oil Red $\mathrm{O}$ or Schiff staining), and the area occupied by tissue with positive staining was quantified semi-automatically by ImageJ. Data were analyzed as the percent of non-empty area occupied by tissue with positive staining. 


\subsubsection{Tissue Preparation for Histology}

At P105, animals from Cohort 2 were deeply anesthetized with sodium pentobarbital, and sacrificed by transcardial perfusion with $0.9 \%$ saline, followed by $4 \%$ paraformaldehyde. Brains were removed post-fixed overnight in the same fixative at $4{ }^{\circ} \mathrm{C}$. Brains were then cryoprotected in 30\% sucrose and sectioned at $60 \mu \mathrm{m}$ on a cryostat (Thermo Scientific) in a one-in-four series. Pooled data from two brains per litter per sex was analyzed using the procedures described below.

\subsubsection{Double Immunohistochemistry for ER $\alpha$ and POMC}

Free-floating tissue sections from Cohort 2 animals were washed several times with $0.1 \mathrm{M}$ phosphate buffer (PB) and treated with $3 \% \mathrm{H}_{2} \mathrm{O}_{2}$ in $10 \%$ methanol for 10 minutes at room temperature to quench endogenous peroxidase activity. Sections were then washed and blocked in 1\% bovine serum albumin (BSA) with $0.3 \%$ Triton-X 100 for one hour at room temperature. Sections were then incubated in monoclonal mouse anti-ER $\alpha$ antibody (1:500; DAKO) in the same blocking solution for 72 hours at $4{ }^{\circ} \mathrm{C}$ under gentle agitation. Slices were washed and incubated with biotinylated donkey antimouse secondary antibody (1:200; Jackson Immunoresearch) for one hour followed by incubation in avidin-biotin horseradish peroxidase complex (Vectastain Elite ABC kit), and finally developed in $0.05 \% 3,3^{\prime}$-diaminobenzidine (DAB) enhanced with $0.05 \%$ nickel ammonium sulphate and $0.05 \%$ cobalt chloride with $0.05 \% \mathrm{H}_{2} \mathrm{O}_{2}$, yielding a blue reaction product. Sections were washed thoroughly, blocked for one hour and then incubated with rabbit anti-POMC antibody (1:10,000; Phoenix Pharmaceuticals) for $24 \mathrm{~h}$ at room temperature in the same blocking solution. Sections were washed and subsequently incubated in biotinylated donkey anti-rabbit secondary antibody (1:200; Jackson Immunoresearch) for one hour. The avidin-biotin complex and DAB reaction was carried out as before except with no metal enhancement yielding a brown DAB precipitate. Slices were mounted on gelatin subbed slides, dehydrated and coverslipped with Permount (Fisher Scientific).

\subsubsection{Immunofluorescence for Vesicular Glutamate Transporter 1 (vG- lut) or Glutamic Acid Decarboxylase 67 (GAD-67) and POMC, and AgRP}

Tissue sections from Cohort 2 animals were processed for either vGlut1 and POMC, or GAD-67 and POMC immunofluorescence. Sections were washed several times in $0.1 \mathrm{M} \mathrm{PB}$, then incubated in primary antibody cocktails contained rabbit anti-POMC (1:2500, Phoenix Pharmaceuticals) and either mouse anti-vGlut1 (1:1000, Millipore) or mouse anti-GAD67 (1:500, Millipore) for 24 hours at room temperature under gentle agitation. The following day, sections were washed and then incubated for one hour in 
a cocktail consisting donkey anti-mouse Alexa Fluor 594 conjugated IgG and donkey anti-rabbit Alexa Fluor 488 conjugated IgG (1:200, both from Life Technologies). Sections were mounted on glass slides and coverslipped using Prolong Gold containing 4',6-diamidino-2-phenylindole (DAPI) as a nuclear counterstain (Life Technologies). Sections stained for AgRP were processed as above with goat anti-AgRP (1:1000, Santa Cruz Biotechnology) and donkey anti-goat Alexa Fluor 594 IgG (1:200, Life Technologies).

\subsubsection{Stereology}

Sections from Cohort 2 double-stained for ER $\alpha$ and POMC were analyzed using unbiased stereology to estimate the total number of ER $\alpha$-, POMC-, and double-stained cells in the ARC. First, the ARC was outlined bilaterally at low magnification (4x) through sections encompassing the anterior and posterior extent of the nucleus using Stereoinvestigator Software (MicroBrightField). The optical fractionator method was used to estimate the total number of cells in the region of interest at high magnification (60x). Dissector frames of $80 \mu \mathrm{m}^{2}$ were placed along a $100 \mu \mathrm{m}$ grid within each contour. The dissector height was set at $15 \mu \mathrm{m}$. Counts for each type of cell were estimated by StereoInvestigator software.

\subsubsection{Quantification of vGlut1 and GAD67 Putative Synaptic Contacts}

Sections from Cohort 2 animals were analyzed at 100x magnification using an Olympus BX61 microscope equipped with an Olympus DSU disk-scanning confocal unit. Confocal images of POMC perikarya were captured using InVitro software (Media Cybernetics), and analyzed using ImageJ (National Institutes of Health). POMC cell perimeter, plus the number of vGlut1 and GAD67 containing puncta in close proximity to the POMC perikarya was quantified in at least 10 POMC cells each per animal.

\subsubsection{Quantification of POMC and AgRP Projections}

DAPI nuclear counterstaining was used to identify the neurosecretory portion of the paraventricular nucleus ( -0.70 to $-0.94 \mathrm{~mm}$ from Bregma) [272]. Using POMC and AgRP stained sections from Cohort 2 animals, Z-stacks at $1 \mu \mathrm{m}$ intervals through the PVN were collected at low power (10x) using an Olympus BX61 microscope equipped with an Olympus DSU disk-scanning confocal unit. At least two bilateral Z-stacks were captured per animal. ImageJ was used to collapse Z-stacks into composite images. These composite images were binarized and skeletonized using identical parameters for each image. DAPI nuclear counterstaining was used to outline the borders of the nucleus, and fibre density within the area of interest was quantified automatically by the measure of area fraction (relative number of labelled pixels vs. total pixels in area 
of interest). These values were then expressed as a percent of the same-sex Control animals.

\subsubsection{6 qRT-PCR}

One animal per sex and post-natal diet were randomly selected from each litter in Cohort 1 for analysis of gene expression using qRT-PCR, except where prohibited by attrition or estrus status. Micropunches of the ARC were homogenized, and RNA was isolated in $500 \mu \mathrm{l}$ TRIzol reagent (Life Technologies). Reverse-transcription was performed using the SuperScript II kit with oligo(DT) primer and the method provided by the manufacturer (Life Technologies). Real-Time PCR was performed in a MyiQ Single Colour Real-Time PCR Detection System (Bio-Rad) using iQ SYBR Green Super Mix (Bio-Rad). Gene expression was determined relative to the housekeeping control genes GAPDH and $\beta$-Actin using the $2^{-\Delta \Delta C t}$ method [273]. Control males consuming standard chow were used as the control group for all qRT-PCR analysis, thereby allowing for the detection of sexually dimorphic gene expression. All primer pairs were tested for efficiency over five orders of magnitude of template concentration. Primer sequences are listed in Table A.2.

\subsubsection{Statistics}

To avoid confounds due to litter effects, littermates of the same sex and post-natal diet were pooled in all statistical analyses as recommended by Holson \& Pearce ([163]). For Cohorts 1 and 2, this typically meant that at least two animals per sex per litter per diet were analyzed and pooled, except in the case of qRT-PCR analysis. Because pooled animals are treated as technical replicates of one another, this approach has the advantage of yielding more accurate measures. This approach also eliminates the problem of pseudoreplication because each litter is treated as the unit of analysis. Data are expressed in graphs as mean \pm SEM. Data were analyzed using two-way ANOVAs with sex and perinatal treatment as factors. Where applicable, post-natal diet was included as an additional factor, yielding a three-way design. Indirect calorimetry data were also analyzed using a repeated-measures ANOVA to compare pre- and post-high-fat diet parameters. Significant main or interaction effects were further analyzed using Fisher's Protected LSD with a significance criterion set at $p<0.05$. Planned comparisons between BPA-treated animals and both Control and DES-treated animals of the same sex were conducted as a matter of course whenever justified by a treatment by sex interaction. 


\subsection{RESULTS}

\subsubsection{BPA Exposure does not Affect Maternal Body Weight or Food Intake}

Exposure to BPA or DES did not affect maternal food intake or body weight at any point during gestation and lactation (Tables 3.1, 3.2). Pregnant dams consumed an average of 0.19 and $3.49 \mu \mathrm{g} / \mathrm{kg} / \mathrm{day}$ of BPA in the 0.001 and $0.02 \mathrm{ppm} \mathrm{BPA} \mathrm{treatment}$ groups respectively. Across postnatal days $0-13$, dams consumed an average of 0.36 and $7.2 \mu \mathrm{g} / \mathrm{kg} / \mathrm{day}$ of BPA in the 0.001 and $0.02 \mathrm{ppm} \mathrm{BPA} \mathrm{treatments} \mathrm{respectively}$ (Table 3.3). Dams in the DES treatment consumed an average of $0.64 \mu \mathrm{g} / \mathrm{kg} /$ day of DES during pregnancy, and $1.41 \mu \mathrm{g} / \mathrm{kg} /$ day during lactation (Table 3.3). Because pups begin reaching for solid food as of P14, dose became difficult to estimate during the last week of lactation. As such, we give data based on whole-cage food disappearance, but we do not attribute this to maternal food intake alone (Table 3.3).

\subsubsection{BPA-Exposed Female Offspring Become Hyperphagic and Obese When Fed a High-Fat Diet in Adulthood}

At 3 months of age we began feeding a subset of offspring ad libitum high-fat diet until the time of sacrifice at 5.5 months of age. Maternal treatment did not affect body weight in either male or female offspring at 3 months of age while animals consumed standard lab chow, nor were there any differences in baseline body weight among animals who would later be switched to the high-fat diet (Figure 3.2a, 3.2b). When comparing body weight at the time of sacrifice to baseline weight prior to high-fat diet exposure, we found a significant effect of $\operatorname{sex}\left(F_{(1,53)}=89.94, p<0.001\right)$, maternal treatment $\left(F_{(3,53)}=3.38, p<0.05\right)$, and postnatal diet $\left(F_{(1,53)}=48.05, p<0.001\right)$. Males exposed to the high-fat diet were all significantly heavier than their chow-consuming counterparts at the time of sacrifice $(p<0.01)$, but this effect did not vary according to maternal treatment (Figure 3.2a). When females exposed to BPA at $0.02 \mathrm{ppm}$ were made to consume the high-fat diet, they became significantly heavier than their high-fat diet-consuming Control and DES-exposed groups $(p<0.001$ and $p<0.05$ respectively; Figure 3.2b). There was a significant effect of $\operatorname{sex}\left(F_{(1,52)}=4.6, p<0.05\right)$, maternal treatment $\left(F_{(1,52)}=2.89, p<0.05\right)$, and postnatal diet $\left(F_{(1,52)}=43.74, p<\right.$ 0.001) on average daily caloric intake across the period of high-fat diet exposure (Figure 3.2). Females exposed to BPA at $0.02 \mathrm{ppm}$ consumed significantly more calories per day than Control females on the same diet $(p<0.05$, Figure 3.2d).

After sacrifice, we measured the weight of several fat pad pads in experimental animals. We found a significant main effect of $\operatorname{diet}\left(F_{(1,53)}=50.88, p<0.001\right)$ as well as a significant interaction between sex, diet, and maternal treatment $\left(F_{(3,53)}=\right.$ $4.88, p<0.01$ ) in the weight of the perigonadal fat pad (Figure 3.3). High-fat diet 
consuming females exposed to BPA at $0.02 \mathrm{ppm}$ had significantly more perigonadal fat than Control and DES-treated females on the same diet ( $p<0.001$ for both), as well as females exposed to the same dose of BPA but left to consume standard chow ( $p<0.001$; Figure 3.3d). The retroperitoneal fat pad showed a similar pattern, with significant main effects of $\operatorname{sex}\left(F_{(1,53)}=89.08, p<0.001\right)$, maternal treatment $\left(F_{(3,53)}=4.56, p<0.01\right)$, diet $\left(F_{(1,53)}=92.58, p<0.001\right)$, as well as a significant interaction between sex and $\operatorname{diet}\left(F_{(1,53)}=10.56, p<0.01\right.$; Figure 3.3). The retroperitoneal fat pad was significantly heavier in high-fat diet-consuming females exposed to BPA at $0.02 \mathrm{ppm}$ when compared to Control animals on the same diet $(p<0.01)$, as well as compared to female counterparts consuming standard chow $(p<0.001$; Figure 3.3d). We also found that the weight of the retroperitoneal fat pad was increased in males exposed to BPA at $0.02 \mathrm{ppm}(\phi<0.05)$, but only while they were consuming chow (Figure 3.3a). We found significant main effects of sex $\left(F_{(1,53)}=34.76, p<0.001\right)$, maternal treatment $\left(F_{(3,53)}=3.26, p<0.05\right)$, and diet $\left(F_{(1,53)}=98.45, p<0.001\right)$ in the subcutaneous white adipose tissue depot (Figure 3.3). Females exposed to BPA at $0.02 \mathrm{ppm}$ and subjected to high-fat diet-induced obesity had significantly larger subcutaneous depots than Control females on the same diet $(p<0.01)$, as well as females exposed to the same BPA treatment but left to consume chow $(p<0.001$; Figure 3.3d). Finally, intrascapular brown adipose tissue (BAT) was significantly affected by $\operatorname{sex}\left(F_{(1,53)}=106.18, p<0.001\right)$ and maternal treatment $\left(F_{(3,53)}=4.53, p<0.05\right)$. There was also a significant interaction between sex, maternal treatment, and diet $\left(F_{(3,53)}=3.02, p<0.05\right.$; Figure 3.3). The brown adipose tissue depot was significant larger in females exposed to BPA at $0.02 \mathrm{ppm}$ when fed a high-fat diet as compared to Control females on the same diet $(p<0.05$; Figure 3.3d). Interestingly, males exposed to BPA at $0.02 \mathrm{ppm}$ showed significantly elevated brown adipose tissue depot weights compared to Control and DES-treated animals $(p<0.01$ for both), but only while consuming the chow diet (Figure 3.3a).

\subsubsection{BPA Exposure Affects Energy Expenditure in Males}

\section{Baseline Parameters at Two Months}

At 2 months of age, and before placing them on ad libitum high-fat diet, we used indirect calorimetry to measure a number of metabolic parameters in our animals. Though we measured $\mathrm{VO}_{2}$ and $\mathrm{VCO}_{2}$, we elected to use these values to compute average energy expenditure, and the respiratory exchange ratio (RER; see Method 3.2.5). Analysis of average hourly energy expenditure showed a significant interaction between sex and maternal treatment both at night $\left(F_{(3,29)}=5.42, p<0.001\right)$ and during the day $\left(F_{(3,53)}=5.77, p<0.01\right)$. We saw a consistent pattern of elevated energy expenditure across the $24 \mathrm{~h}$ period of measurement in males exposed to BPA at $0.02 \mathrm{ppm}$ (Figure 3.4a) and significantly elevated rates of energy expenditure during both the dark and 
light phases of the light/dark cycle $(p<0.01$ vs. Control males, $p<0.05$ vs DES males; Figure 3.4b). We also found a significant interaction between sex and maternal treatment in daytime RER $\left(F_{(3,29)}=4.57, p<0.05\right)$. Post-hoc analysis indicated that males exposed to BPA at $0.02 \mathrm{ppm}$ had significantly higher RER values than Control males $(p<0.01$; Figure 3.4e). This is indicative of a somewhat greater tendency toward carbohydrate metabolism in these animals. The indirect calorimetry equipment also recorded locomotor behaviour in terms of infrared beam breaks. We did not see any significant differences in locomotion across the $24 \mathrm{~h}$ measurement period in any animals (Figures 3.4g, 3.4h.)

\section{Metabolic Parameters Post-High-Fat Diet Exposure}

After we placed a subset of animals on the high-fat diet and allowed sufficient time for the development of diet-induced obesity, we returned all of the animals to the indirect calorimeters. For the animals that remained on chow, this constituted their first measurement in the units. For animals that had been switched to the high-fat diet, this was their second time being measured and so their baseline data recorded at 2 months of age complements the post-high-fat diet data reported here.

We found that males exposed to $0.02 \mathrm{ppm} \mathrm{BPA}$ showed significantly elevated average hourly energy expenditure across the $24 \mathrm{~h}$ period $(p<0.001$ vs. Control, $p<0.01$ vs. DES), but only when consuming chow. The tendency for these animals to exhibit increased energy expenditure was suppressed by diet-induced obesity, for these animals showed no difference in energy expenditure compared to their Controls. We saw a significant effect of diet on RER both at night $\left(F_{(1,52)}=244.47, p<0.001\right)$ and during the day $\left(F_{(1,52)}=155.52, p<0.001\right.$; Figures 3.5c, 3.5d). All animals exposed to the high-fat diet showed the expected reduction in RER that indicates a shift toward fat as a fuel source. Despite varying rates of diet-induced obesity, this value was not affected by sex or maternal treatment. Locomotor behaviour was generally reduced when high-fat diet consuming animals were compared to their earlier baseline measures by repeated-measures ANOVA $\left(F_{(1,25)}=171.16, p<0.001\right.$ by Greenhouse-Geisser correction; Figures 3.5e, 3.5f). Since locomotion was not different at this time point between diets ( $\phi>0.05$ for the main effect of diet), the repeated-measures effects are likely a reflection of age.

\subsubsection{Males Exposed to BPA Have Impaired Glucose Tolerance}

At approximately 5 months of age, we subjected both chow- and high-fat diet-consuming animals to an intraperitoneal glucose tolerance test. This test was preceded by an overnight fast, so baseline measures reflect fasting blood glucose. Males consuming the high-fat diet exposed to BPA at $0.02 \mathrm{ppm}$ had elevated blood glucose at this baseline measure ( $p<0.05$ vs. Control). Subsequent to the glucose injection, 0.02 
ppm BPA-exposed males showed hyperglycaemia relative to their Controls regardless of diet at several post-injection timepoints (Figure 3.6c). In contrast, neither BPA nor DES affected baseline or post-injection blood glucose levels in female animals consuming either diet. In fact, BPA- and DES-exposed females consuming chow were actually hypoglycaemic compared to Controls 15 minutes post-injection (Figure 3.6d). When data were analyzed by area under the curve (AUC) we found a main effect of $\operatorname{sex}\left(F_{(1,50)}=60.06, p<0.001\right)$, treatment $\left(F_{(3,50)}=5.97, p<0.001\right)$, $\operatorname{diet}\left(F_{(1,50)}=18.26, p<0.001\right)$, and an interaction between sex and treatment

$\left(F_{(3,50)}=3.32, p<0.05\right)$. Males, regardless of diet, showed significantly higher AUC values than Controls if they had been exposed perinatally to BPA at $0.02 \mathrm{ppm}(\not<0.01$ vs. Control \& DES males consuming the same diet; Figure 3.6a).

\subsubsection{Hepatic Lipid and Glycogen Content is Altered by Diet-Induced Obesity}

High-fat diet-induced obesity lead to marked increases in hepatic content, as inferred by positive Oil Red $\mathrm{O}$ staining (main effect of diet $F_{(1,54)}=52.54, p<0.001$; Figure 3.7a). This effect was not different between sexes $\left(F_{(1,54)}=0.43, p>0.05\right)$, treatments $\left(F_{(3,54)}=0.75, p>0.05\right)$, nor were there any interactions between any of these factors. Hepatic glycogen content revealed by periodic acid-Schiff staining showed a marked decline in response to prolonged high-fat diet feeding, though interestingly this only occurred in male animals $\left(F_{(1,54)}=26.06, p<0.001\right.$ interaction between sex and diet; Figure 3.7b).

\subsubsection{BPA-Exposed Females Develop Hyperleptinemia on a High-Fat Diet}

Prolonged exposure to the high-fat diet had the expected effect of promoting obesity, and increasing plasma leptin levels in both males and females, with the exception of female DES-exposed mice (main effect of diet $F_{(1,50)}=135.62, p<0.001$; Figure 3.8). Additionally, we found significant main effects of $\operatorname{sex}\left(F_{(1,50)}=64.42, p<0.001\right)$, treatment $\left(F_{(3,50)}=4.30, p<0.01\right)$, as well as a significant three-way interaction between sex, treatment, and diet $\left(F_{(3,50)}=5.51, p<0.01\right)$. We explored this interaction further, and found that females exposed to BPA at $0.02 \mathrm{ppm}$ and subject to diet-induced obesity had significantly elevated leptin relative to Control females on the same diet $(p<0.001$; Figure 3.8).

\subsubsection{BPA Exposure Affects Circulating Resistin and Insulin Levels on a High-Fat Diet}

Unfasted insulin levels collected at the time of sacrifice were significantly affected by $\operatorname{sex}\left(F_{(1,50)}=61.13, p<0.001\right)$ and $\operatorname{diet}\left(F_{(1,50)}=10.18, p<0.01\right)$, with a sig- 
nificant interaction between sex, treatment, and $\operatorname{diet}\left(F_{(1,50)}=7.27, p<0.001\right)$ as well. Regardless of diet or treatment, insulin levels were generally much lower in females than in males. Diet-induced obese males exposed either to DES, or BPA at 0.02 ppm were hyperinsulinemic compared to Control males on the same diet $(p<0.01$ and $p<0.001$ respectively; Table 3.4). Resistin levels were significantly affected by $\operatorname{sex}\left(F_{(1,50)}=5.11, p<0.05\right)$, treatment $\left(F_{(3,50)}=3.28, p<0.05\right)$, and increased by high-fat diet exposure $\left(F_{(1,50)}=13.06, p<0.001\right)$. We found a three-way interaction between the aforementioned factors $\left(F_{(3,50)}=3.15, p<0.05\right)$, which upon post-hoc analysis revealed a pattern similar to what we observed in leptin (Table 3.4). Diet-induced obese females exposed to BPA at $0.02 \mathrm{ppm}$ had elevated resistin levels as compared to Control animals on the high-fat diet $(p<0.01)$.

\subsubsection{Reduced POMC Projections to the PVN in BPA-Exposed Animals}

Analysis of POMC projections into the PVN in offspring sacrificed at 3 months of age revealed a significant effect of treatment $\left(F_{(3,20)}=4.77, p<0.05\right)$ and an interaction between sex and treatment $\left(F_{(3,20)}=3.00, p<0.05\right)$. Male and female mice exposed to BPA at $0.02 \mathrm{ppm}$ had projection densities that were respectively $62.66 \%$ and $91.99 \%$ of their same-sex Controls (Figure 3.9a). DES-exposed females had POMC projection densities that were $77 \%$ of Controls. Immunofluorescence analysis of AgRP projections into the PVN revealed a significant main effect of treatment $\left(F_{(3,14)}=3.55, p<0.05\right)$, accounted for by increased projection density in DESexposed animals $(128.92 \%$ and $143 \%$ of same-sex controls in males and females respectively, Figure 3.9d).

\subsubsection{The Balance of Excitatory and Inhibitory Synapses onto POMC Neurons is Sexually Dimorphic, but not Affected by BPA or DES Treatment}

Confocal analysis of GAD67-expressing puncta on POMC neuron perikarya, taken as putative inhibitory synapses, did not reveal any sex- or treatment-related differences. Putative excitatory synapses, as revealed by confocal analysis of vGlut1 immunofluorescence, were significantly different between sexes $\left(F_{(1,28)}=22.16, p<0.001\right)$. Treatment did not influence this effect $(\phi>0.05$ for interaction; Figure 3.9g).

\subsubsection{The Proportion of POMC Neurons that Co-Express ER $\alpha$ is Sexu- ally Dimorphic, and Increased by BPA Exposure}

We used unbiased stereology to count ER $\alpha$ and immunopositive cells in the ARC, as well as cells that co-express these proteins. Analysis of total cell count estimates for each type revealed a significant effect of treatment on ER $\alpha$ immunpositive cells 
$\left(F_{(3,17)}=3.23, p<0.05\right)$. Females exposed to BPA at $0.001 \mathrm{ppm}$ had significantly more ER $\alpha$ immunopositive cells than their Controls $(p<0.05$; Figure 3.10a). The main effect of sex did not reach statistical significance here, but it is worth noting that Control females tended to have fewer of such cells than males (333.25 \pm 104.17 and $689.70 \pm 178.11$ respectively). There was a significant main effect of sex in the number of POMC immunopositive neurons $\left(F_{(1,17)}=35.46, p<0.001\right)$, with counts in females roughly double those in males (Figure 3.10b). There were significant effects of $\operatorname{sex}\left(F_{(1,17)}=7.80, p<0.05\right)$ and treatment $\left(F_{(3,17)}=3.84, p<0.05\right)$ on the percent of POMC neurons that co-expressed ER $\alpha$. Males generally had more of these neurons $(p<0.05)$, but the difference was more pronounced when compared to Control females. Animals exposed to BPA at 0.001 and $0.02 \mathrm{ppm}$ had a significantly higher proportion of these double-labelled cells than Control animals, and this effect was most pronounced in females (Figure 3.10c).

\subsubsection{BPA Exposure Affects the Transcriptional Response to Diet-Induced Obesity}

We used qRT-PCR to analyze gene expression in the ARC in animals sacrificed at 5.5 months of age. Half of these animals were subjected to diet-induced obesity. We found a significant main effect of $\operatorname{sex}\left(F_{(1,47)}=6.62, p<0.05\right)$, as well as a significant threeway interaction between sex, treatment, and $\operatorname{diet}\left(F_{(3,47)}=3.01, p<0.05\right)$. As with our immunohistochemistry results, females exposed to BPA at $0.001 \mathrm{ppm}$ had significantly greater levels of ER $\alpha$ expression than their Controls, but only when consuming chow (Figure 3.11a). Females exposed to BPA at $0.02 \mathrm{ppm}$ showed a significant elevation in $\mathrm{ER} \alpha$ expression in response to diet-induced obesity $(p<0.05$ vs. Control females consuming the high-fat diet, $p<0.05$ vs. females exposed to the same dose but consuming chow; Figure 3.11a). POMC expression was generally increased by highfat diet exposure $\left(F_{(1,54)}=4.15, p<0.05\right)$, though this relationship was complicated by a three-way interaction between sex, treatment and $\operatorname{diet}\left(F_{(3,54)}=2.76, p<0.05\right)$. Closer inspection of these values revealed a substantial diet-induced upregulation of POMC present in Control females, but attenuated in females exposed to BPA at 0.001 $\mathrm{ppm}$, and completely absent in those exposed to BPA at $0.02 \mathrm{ppm}$ (Figure 3.11b). POMC upregulation was similarly absent in DES-exposed females, but this is perhaps a consequence of the fact that they were not hyperleptinemic at the time of sacrifice (see Figure 3.8). AgRP expression was significantly upregulated in high-fat diet consuming males exposed to BPA at $0.02 \mathrm{ppm}$ as compared to Control males on the same diet, and males from the same treatment but consuming chow $(\phi<0.05$; Figure 3.11c). We observed a similar trend in NPY expression, but the effect did not reach statistical significance $(p=0.07$; Figure 3.11c). 


\subsection{DISCUSSION}

At ecologically relevant doses, BPA is capable of exerting acute, activational effects in adult animals, as well as organizational effects in developing animals. All other things being equal, the prospect of organizational changes induced by BPA is of greater concern, because these are likely to persist long beyond the initial period of exposure. In this study, we have shown that perinatal BPA exposure at two environmentally relevant doses has effects in adult animals that remain long after the period of initial exposure. The perinatal period-here defined as the period encompassing an animal's entire prenatal and postnatal life up until the time of weaning - is a time of extreme sensitivity to exogenous effects on organogenesis, sexual differentiation, and neural development, all of which take place early in life. In mammals, this period is characterized by absolute dependence on maternally-derived nutrition, either through the placenta in-utero or in the milk postnatally. BPA crosses the placental barrier with relative ease [35], and it also insinuates itself into the milk [38], thereby gaining access to the developing organism at all of its most vulnerable stages.

In this study, we found few metabolic effects among male and female offspring exposed to BPA at $0.001 \mathrm{ppm}$. These findings confirm and extend those of Ryan et al., which used the same dose and similar methodology [143]. On the other hand, animals exposed to our higher dose of BPA at $0.02 \mathrm{ppm}$ showed a number of sex-specific adverse metabolic and neurobiological outcomes. Consistent with the findings of Wei et al., [144] male mice exposed to BPA at $0.02 \mathrm{ppm}$ showed impaired glucose tolerance in adulthood, and this condition was exacerbated by exposure to a high-fat diet. In contrast, our male BPA-exposed mice did not show an elevated tendency toward diet-induced obesity compared to Control animals—all readily developed obesity on the high-fat diet. Our inability to observe a tendency toward diet-induced obesity may actually be a product of our model of high-fat diet exposure. Male CD-1 mice readily develop diet-induced obesity when placed on a high-energy diet, and the physical limitations associated with rapid fat accumulation might have yielded a ceiling effect in our study. It may be that a treatment effect on obesity would have emerged had we elected to use a diet with a more moderate energy content (diets with $40 \%$ calories from fat are a common choice). In a similar vein, rats such as those used by Wei et al., can carry substantial amounts of fat relative to their lean body mass, and may have a higher ceiling for the development of diet-induced obesity. Intact female mice, on the other hand, tend to resist diet-induced obesity. Indeed, our Control females gained only slightly more weight on the high-fat diet than did Control females who remained on standard chow. Females exposed to BPA at $0.02 \mathrm{ppm}$ were the exception to this, quickly developing diet-induced obesity on the high-fat diet. In spite of the tendency toward obesity in these animals, we did not see any effect on glucose tolerance, which remained at levels equivalent to Control animals. Female mice are generally more glu- 
cose tolerant than males, even when important metabolic sensors such as leptin are disabled [235]. Females with physiological levels of circulating $17 \beta$-estradiol maintain normal levels of glucose tolerance, even in the context of diet-induced obesity [274]. This fact may account for the apparent lack of treatment effect on glucose tolerance in our females, and may imply that the metabolic effects of estrogen signalling remained intact in our animals.

Under conditions of energy excess, increases in circulating leptin stimulate POMC expression that in turn organizes a reduction in food intake designed to restore the organism to homeostasis $[176 ; 178 ; 275]$. In a state of leptin resistance brought on by protracted high-fat diet exposure, this sequence of events no longer takes place. High-fat diet exposed males were uniformly hyperleptinemic and had POMC mRNA levels that were equivalent to their chow-consuming counterparts. Diet-induced obese females exposed to BPA at $0.02 \mathrm{ppm}$ were, in contrast to their Controls, hyperleptinemic but with no increase in POMC transcription. Control females consuming the high-fat diet were moderately hyperleptinemic but they also exhibited correspondingly upregulated POMC expression. This suggests indirectly that leptin's negative feedback effects on feeding were functioning correctly in Control females. Because we did not detect any alterations in the balance of excitatory and inhibitory synapses onto POMC neurons, it is not likely that the observed differences in expression were due to alterations in local innervation of POMC neurons [182;181]. It is also interesting to note that in male mice exposed to BPA at $0.02 \mathrm{ppm}$, the orexigenic transcripts AgRP and NPY were upregulated by diet-induced obesity. Under ordinary conditions, these neuropeptides are expected to be downregulated by leptin as part of its negative feedback regulation of feeding [276].

Given that perinatal exposure to BPA and DES is known to alter ER $\alpha$ expression in various hypothalamic nuclei $[277 ; 278 ; 269]$, and that ER $\alpha$ expression in the ARC is important for the regulation of metabolic homeostasis [182; 279], we reasoned that the obesogenic effects of BPA may be mediated by organizational effects on the development of these cells. ER $\alpha$ immunopositive cells showed a sexually dimorphic pattern of expression with Control females having fewer than males by half. Females exposed to $\mathrm{BPA}$ at $0.001 \mathrm{ppm}$ had significantly more ER $\alpha$ immunopositive cells than female Controls, a finding that is mirrored by our qRT-PCR results and consistent with previously published reports on the effect of BPA on ER $\alpha$ expression $[277 ; 278 ; 269]$. POMC neurons also showed a sexually dimorphic pattern of expression. Females had significantly more POMC immunopositive neurons than their male counterparts, but this was not affected by any of the treatments. Importantly, the biological effects of leptin appear to synergize with estrogen, such that intact females show a stronger metabolic response to it than intact males [280; 281;282]. Cells that co-express ER $\alpha$ and POMC are known to be involved in the anorectic effects of the estrogens, including their ability to upregulate POMC expression [182; 279; 237]. We hypothesized that BPA-exposed 
animals, showing sex-specific metabolic abnormalities, would have fewer of these cells than Control animals. In fact, we observed the opposite, with animals exposed to both doses of BPA possessing a higher proportion of double-labelled cells than their Controls. Since there was a pronounced sexual dimorphism in the proportion of these cells-Control females having far fewer- that appeared to be reduced by BPA treatment, it may be that the BPA-exposed females have been masculinized in this regard. On the other hand, DES-exposed females did not show the same effect, and so it might be reasonably concluded that its developmental origins are not a direct consequence of ER agonism.

Exposure to the higher dose of BPA resulted in significantly reduced POMC fibre staining in the PVN. Though we observed a significant effect of treatment, and reduced fibre density in both male and female BPA-treated animals, the effect was most pronounced in males. DES-exposed females were similarly affected, though the origin of sex differences here warrants further study. The PVN contains numerous neuroendocrine cell populations that are important effectors of leptin's behavioural and physiological effects. These include neurons expressing thyrotropin-releasing hormone (TRH) [283; 284] and presympathetic neurons with projections innervating the glands and smooth muscles of the viscera [285]. It is like that these observations reflect postnatal disruption of axonal migration, as this pathway is known to develop under the control of leptin during the early postnatal period [286; 202]. These findings are not likely to arise solely from a difference in the number of POMC neurons, as our stereological investigation of the ARC did not reveal any treatment-related differences in their number, nor was there a difference in POMC mRNA expression at baseline.

In our hands, DES-exposed mice did not show any particular propensity toward diet-induced obesity or metabolic defect. This is in contrast to reports demonstrating obesity in mice perinatally exposed to $1 \mu \mathrm{g} / \mathrm{kg} /$ day and $10 \mu \mathrm{g} / \mathrm{kg} /$ day doses of DES [159; 158]. These studies have many differences in their postnatal treatment, the mouse strain used, as well as the means and schedule of exposure to the compound. For these reasons, direct comparability is necessarily limited. The method and dose used by Ryan et al., is virtually the same as ours, and in their hands both male and female DES-exposed mice showed relatively reduced body fat following prolonged high-fat diet treatment [143]. The fact that our diet was more calorically dense $(60 \%$ vs. $40 \% \mathrm{kCals}$ from fat) precluded a complete replication of their findings, certain elements of our data do elaborate upon their observations. DES-exposed females did not develop hyperleptinemia while on the high-fat diet, and had correspondingly low levels of POMC mRNA expression in the ARC. Moreover, these animals had increased $\mathrm{ObRb}$ receptor mRNA in the ARC, as well as increased high-fat diet-induced SOCS3 expression (Figures 3.11e \& 3.11f). These factors may have had a protective effect against diet-induced obesity, and they also suggest that the BPA-exposed phenotype cannot result solely from the estrogenic properties of BPA. 
Early-life DES exposure is known to exert a masculinizing influence on the female brain, enlarging the sexually dimorphic nucleus of the preoptic area (SDN-POA) toward a more male-typical size [156;155]. Though we found sex differences in ER $\alpha$ and POMC expression in the ARC, these did not appear to be reversed by our DES treatment, nor were any other sexually dimorphic characteristics similarly reversed. The ontogeny of these differences is not known, and may depend on hormonally mediated control of apoptosis, as seen in the development of the SDN-POA and anteroventral periventricular nucleus (AVPV) [287], or it may depend on cell fate decisions made during embryonic development [288]. Further study into the normal development of these sex differences will be needed to frame our understanding of the effects of the endocrine disruptors employed here. While DES is acknowledged to be a valid positive control for developmental studies of BPA exposure [150], there exists no satisfactory method for comparing the relative potencies of these chemicals in-vivo, and thus determining a dose optimal for a positive control remains difficult. While both chemicals are famously known as estrogenic, this is a relatively simplistic perspective on the complexity of their biological activities. BPA, for example, is active at a number of nuclear receptors in addition to its varied effects on classical and membrane-bound estrogen receptors (see Section 1.3). It could be that the bulk of BPA's metabolic effects stem not from its estrogenicity, but instead from its actions on some other target.

There exists a certain degree of controversy regarding feed selection in studies of this sort. Throughout the study, we used AIN93G as a Control diet, as well as the basis upon which our treatment diets were based (see Table A.1). In contrast to the adult maintenance diet AIN93M, this diet possesses a relatively higher energy content (derived from fat) in order to support pregnancy, lactation, and early-life growth. Our observations of diet-related effects must be interpreted as comparisons between animals consuming a moderate and extremely high-fat diet. In addition, AIN93G, the high-fat diet, and all of the treatment diets derived from these are composed of purified ingredients that are free of phytoestrogens and other biologically active compounds found in whole foods. This approach was essential, because lot-to-lot variations in phytoestrogen content would prohibit the large-scale, long-lasting experiments that define this project. Nevertheless, a phytoestrogen-free diet is not without its own confounds. In one study, male and female CD-1 mice conceived and raised on high phytoestrogen content diets had reduced body weight, fat, and elevated metabolic rates compared to animals raised on phytoestrogen-free diets [289]. Similar results were obtained by Ruhlen et al.,, again suggesting that low phytoestrogen levels predispose CD-1 mice to the metabolic syndrome in adulthood [290]. These authors found that on E18, male and female fetuses exposed to the low phytoestrogen diet had elevated levels of serum estradiol [290]. On the basis of these findings, it has been suggested that laboratory animals - the outbred CD-1 mice in particular- have acquired through years of controlled breeding and exclusive consumption of corn and soy-based diets 
a basal tolerance to phytoestrogens. When phytoestrogens are withdrawn during development, the fetus is exposed to higher than usual levels of estradiol, perhaps as a compensatory response, and this may program an obesity-prone and reproductively disturbed adult phenotype [290], similar to what is seen in neonatally androgenized mice $[238 ; 250]$. Phytoestrogen withdrawal also has metabolic effects in adult animals, as was recently demonstrated in male Wistar rats [291]. In the weeks following a switch to a phytoestrogen-free diet these rats began to develop obesity, as well as the neurobiological markers thereof including decreased POMC and ER $\alpha$ mRNA and increased AgRP mRNA [291]. It is not clear how either the developmental or adult consequences of phytoestrogen withdrawal might interact with the effects of BPA or DES, nor is it at all clear how such confounds could be avoided without introducing other potential sources of bias.

In this study, we have demonstrated that early-life exposure to BPA programs a sexually dimorphic series of metabolic impairments and neurobiological changes, all of which conspire to bring about an obesity-prone phenotype in adult animals. Given the sexually dimorphic nature of the phenotype presented here, the phenotypic differences observed between DES- and BPA-treated animals, the complexity of BPA pharmacokinetics in-vivo (see Section 1.2), and the multiple receptors for which BPA is a ligand (see Section 1.3), it seems likely that no single mechanism can entirely account for BPA's developmental effects. Further research is required to elaborate on the full scope of targets disrupted by BPA during development. Importantly, our doses of BPA are well within the realm of the ecologically plausible, and well below the EPA reference dose of $50 \mu \mathrm{g} / \mathrm{kg} /$ day. Because of the ubiquity of BPA in the environment, these findings further the case that exposure to this chemical constitutes a risk factor in the developmental origins of metabolic disease. 


\begin{tabular}{lllll}
\hline & Control & BPA $(0.001 \mathrm{ppm})$ & BPA $(0.02 \mathrm{ppm})$ & DES $(0.004 \mathrm{ppm})$ \\
\hline G1-7 & $29.3 \pm 1.01$ & $28.76 \pm 0.88$ & $29.89 \pm 0.58$ & $30.49 \pm 0.63$ \\
G8-14 & $34.02 \pm 0.87$ & $34.32 \pm 0.27$ & $35.09 \pm 0.59$ & $35.07 \pm 0.89$ \\
G14-21 & $51.06 \pm 1.53$ & $48.96 \pm 0.39$ & $51.01 \pm 1.5$ & $49.58 \pm 1.23$ \\
\hline L0-6 & $37.47 \pm 1.32$ & $38.08 \pm 0.9$ & $39.1 \pm 0.61$ & $38.73 \pm 0.98$ \\
L7-13 & $39.64 \pm 1.5$ & $41.52 \pm 1.59$ & $42.62 \pm 0.69$ & $42.18 \pm 1.1$ \\
L14-21 & $38.22 \pm 0.9$ & $40.89 \pm 1.1$ & $41.46 \pm 0.89$ & $40.11 \pm 0.85$ \\
\hline
\end{tabular}

Table 3.1: Maternal body weight across pregnancy and lactation.

Weekly averages of maternal body weight $(\mathrm{g})$ across the gestational and lactational periods.

\begin{tabular}{lllll}
\hline & Control & BPA $(0.001 \mathrm{ppm})$ & BPA $(0.02 \mathrm{ppm})$ & DES $(0.004 \mathrm{ppm})$ \\
\hline G1-7 & $4.82 \pm 0.48$ & $5.81 \pm 1.08$ & $6.32 \pm 1.3$ & $5.23 \pm 1.51$ \\
G8-14 & $6.15 \pm 1.04$ & $7.77 \pm 0.9$ & $6.26 \pm 0.74$ & $5.52 \pm 0.52$ \\
G14-21 & $6.59 \pm 0.27$ & $6.29 \pm 0.44$ & $6.75 \pm 0.41$ & $6.36 \pm 0.53$ \\
\hline L0-6 & $9.86 \pm 1.19$ & $9.71 \pm 0.56$ & $10.55 \pm 0.79$ & $10.39 \pm 0.84$ \\
L7-13 & $16.31 \pm 0.87$ & $17.56 \pm 0.62$ & $17.81 \pm 0.53$ & $17.01 \pm 0.79$ \\
L14-21* & $20.45 \pm 2.05$ & $21.68 \pm 1.42$ & $22.19 \pm 1.22$ & $20.74 \pm 1.2$ \\
\hline
\end{tabular}

Table 3.2: Maternal food intake across pregnancy and lactation.

Weekly averages of maternal food intake (g) across the gestational and lactational periods.

* At P14 pups begin reaching for solid food, so these values represent whole-cage food disappearance.

\begin{tabular}{lllll}
\hline & Control & BPA $(0.001 \mathrm{ppm})$ & BPA $(0.02 \mathrm{ppm})$ & DES $(0.004 \mathrm{ppm})$ \\
\hline G1-7 & 0 & $0.2 \pm 0.03$ & $4.2 \pm 0.84$ & $0.77 \pm 0.15$ \\
G8-14 & 0 & $0.23 \pm 0.03$ & $3.62 \pm 0.43$ & $0.63 \pm 0.06$ \\
G14-21 & 0 & $0.13 \pm 0.01$ & $2.65 \pm 0.14$ & $0.52 \pm 0.04$ \\
Gestational days average & 0 & 0.19 & 3.49 & 0.64 \\
\hline L0-6 & 0 & $0.29 \pm 0.02$ & $6.05 \pm 0.33$ & $1.2 \pm 0.07$ \\
L7-13 & 0 & $0.42 \pm 0.02$ & $8.36 \pm 0.26$ & $1.62 \pm 0.09$ \\
L14-21* & 0 & $0.54 \pm 0.03$ & $10.79 \pm 0.54$ & $2.09 \pm 0.12$ \\
Lactational days 0-13 average & 0 & 0.36 & 7.2 & 1.41 \\
\hline
\end{tabular}

Table 3.3: Estimated maternal doses of BPA and DES.

Weekly doses of each compound ( $\mu \mathrm{g} / \mathrm{kg} /$ day) estimated using maternal food intake and body weight, and that compound's concentration in the diet.

* At P14 pups begin reaching for solid food, so these values represent whole-cage food disappearance. 
(a)

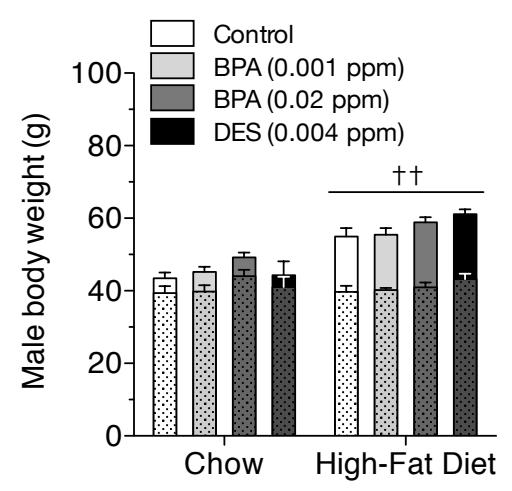

(c)

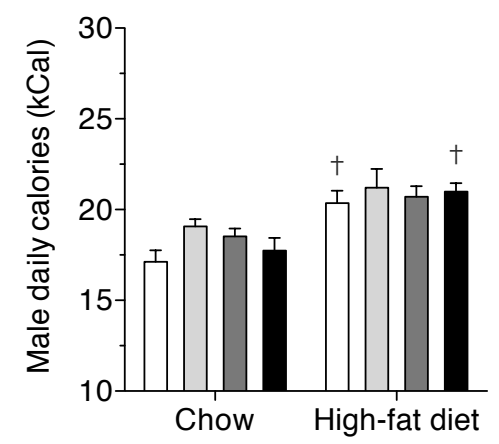

(b)

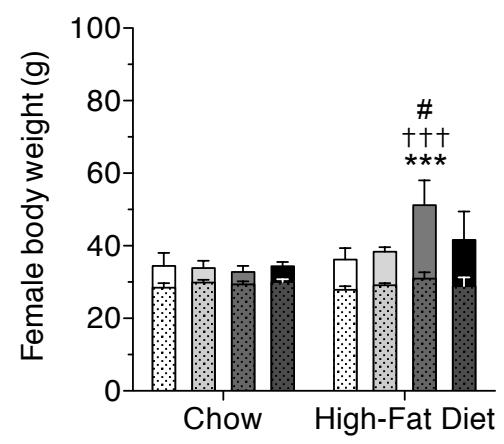

(d)

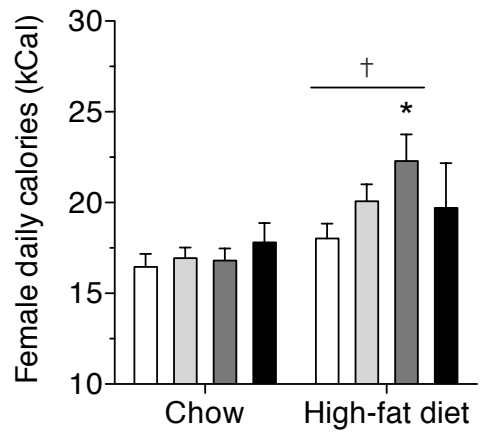

Figure 3.2: Adult body weight and caloric intake.

Male (a) and female (b) body weight at time of sacrifice (baseline weight overlaid). Male (c) and female (d) average daily caloric intake during the period of high-fat diet exposure. $* p<0.05, * * * p<0.001$ vs. control animals of the same sex and group; \#p $<0.05$ vs. DES-treated animals of the same sex and group; $\mathbf{t}_{p}<0.05, \mathbf{t}+p<0.01, \mathbf{t}+\boldsymbol{t}_{p}<0.001$ vs. animals of the same sex and group consuming chow. All values expressed as mean \pm SEM. 
(a)

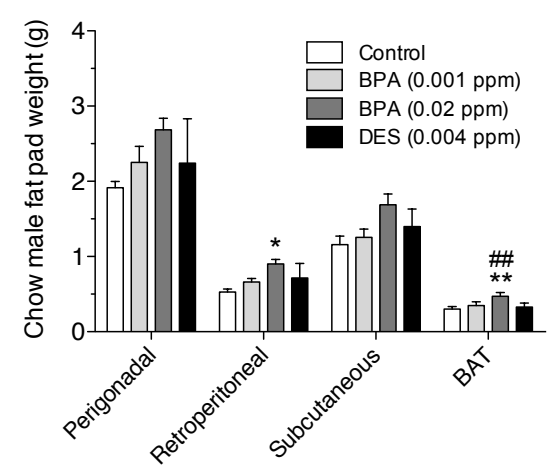

(c)

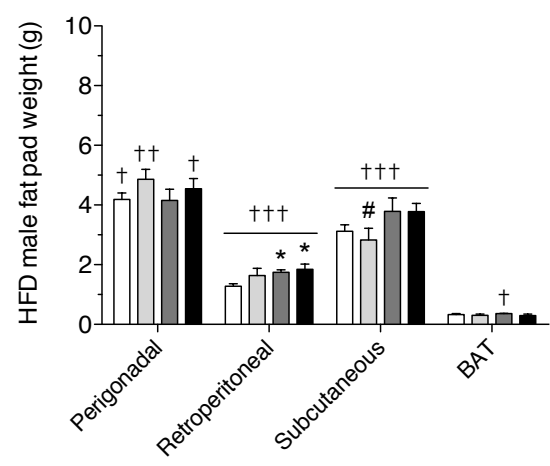

(b)

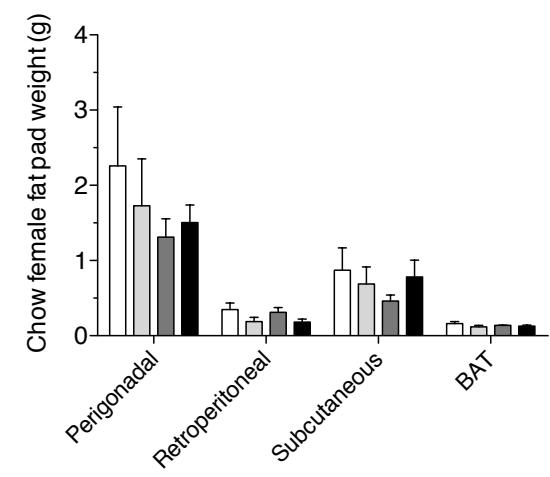

(d)

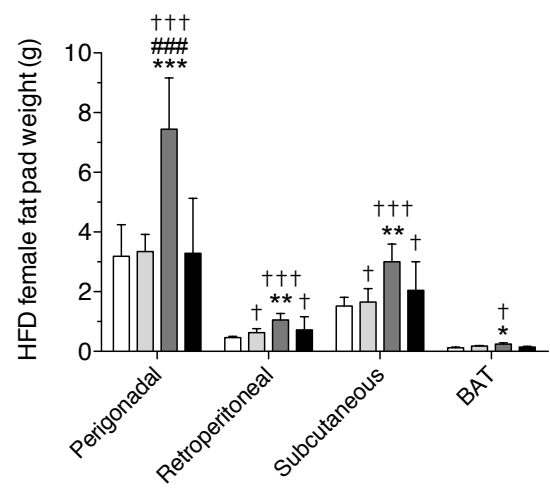

Figure 3.3: Adult fat pad mass.

Weight of the perigonadal, retroperitoneal, subcutaneous white adipose tissue depots, and intrascapular brown adipose tissue (BAT) in male (a) and female (b) chow-consuming adults; male (c) and female (d) high-fat diet consuming adults. $* p<0.05, * * p<0.01, * * * p<0.001$ vs. control animals of the same sex and group; \#p $<0.05$, \#\#p $<0.01$, \#\#\# $<0.001$ vs. DES-treated animals of the same sex and group; $\mathbf{\dagger} p<0.05, \mathbf{t}+p<0.01, \mathbf{t}+\boldsymbol{t}_{p}<0.001$ vs. animals of the same sex and group consuming chow. All values expressed as mean \pm SEM. 
(a)

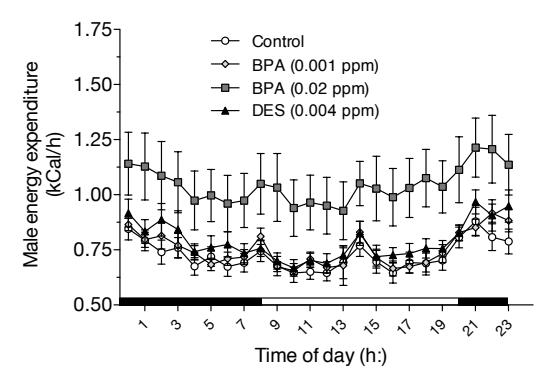

(c)

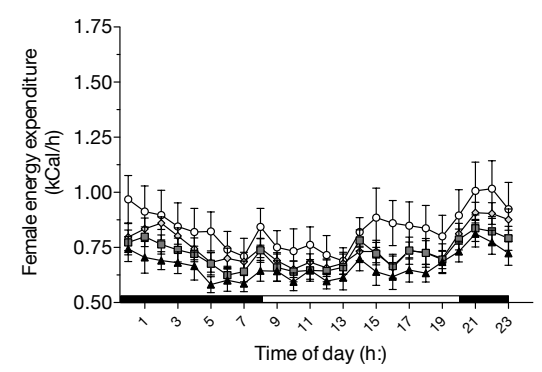

(e)
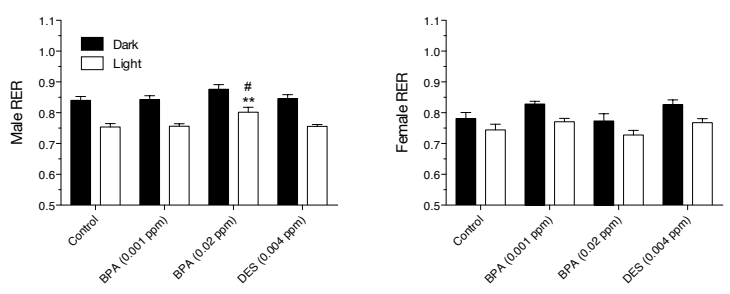

(b)

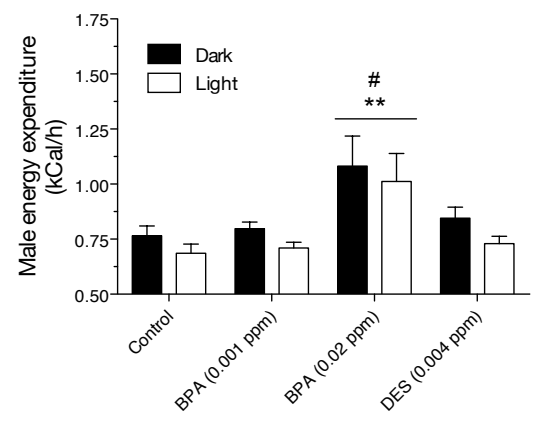

(d)

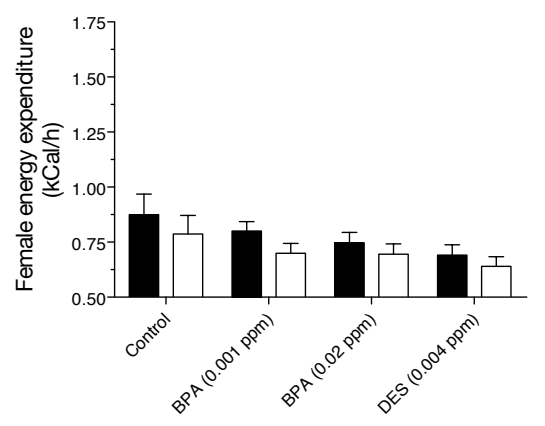

(g)

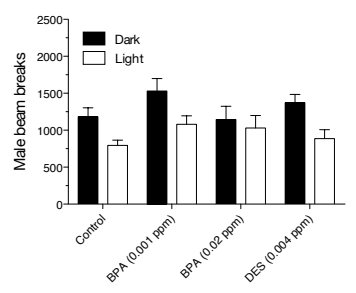

(h)

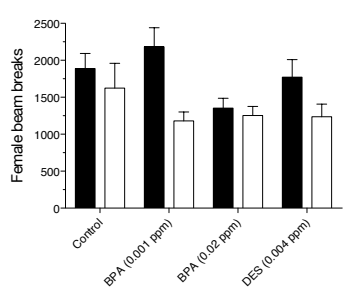

Figure 3.4: Metabolic parameters at two months.

Heat production in males (a,b), and females (c,d) at two months of age. Male (e) and female (f) respiratory exchange ratio (RER), and locomotor activity ( $\mathrm{g} \& \mathrm{~h}$ respectively). All values represent average over $12 \mathrm{~h}$ light or dark period. $* * p<0.01$ vs. control animals of the same sex and group; \#p $<0.05$ vs. DEStreated animals of the same sex and group. All values expressed as mean $\pm \mathrm{SEM}$. 
(a)

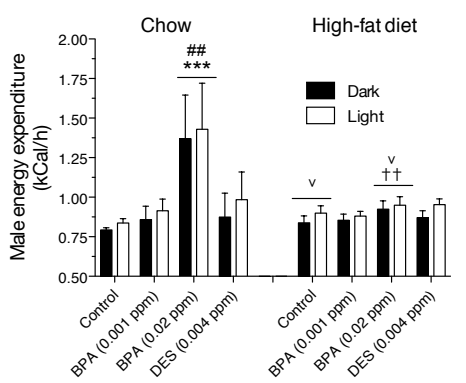

(c)

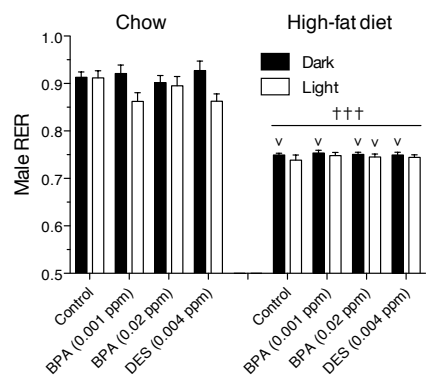

(e)

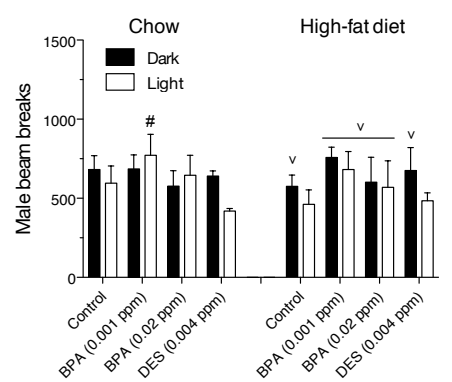

(b)

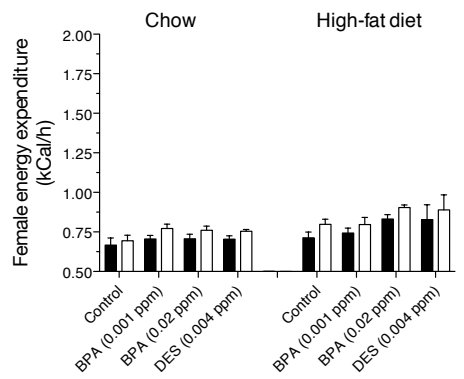

(d)

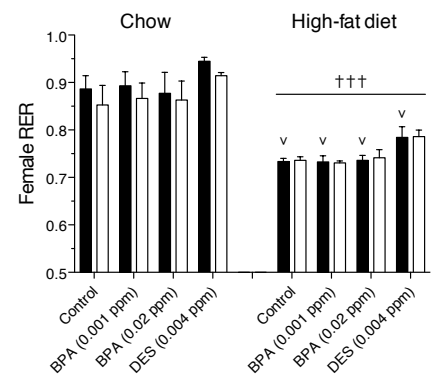

(f)

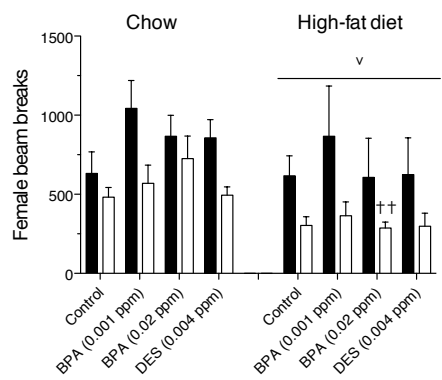

Figure 3.5: Metabolic parameters at five months.

Heat production in male (a) and female (b) animals. Respiratory exchange ratio (RER) in male (c) and female (d) animals. Locomotor activity in male (e) and female (f) animals. All values represent average over $12 \mathrm{~h}$ light or dark period in chow- and high-fat diet-fed animals. $* * * p<0.001$ vs. control animals of the same sex and group; \#p $<0.05, \# \# p<0.01$ vs. DES-treated animals of the same sex and group; $\mathbf{t} p<0.01, \boldsymbol{t}+\boldsymbol{t} p<0.001$ vs. animals of the same sex and group consuming chow; $\vee_{p}<0.05$ vs. repeated measures comparison of same animals pre-high-fat diet exposure. All values expressed as mean \pm SEM. 
(a)

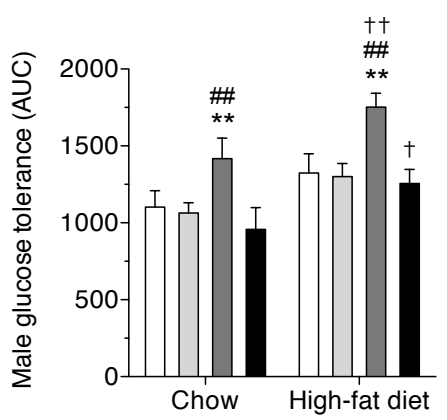

(c)

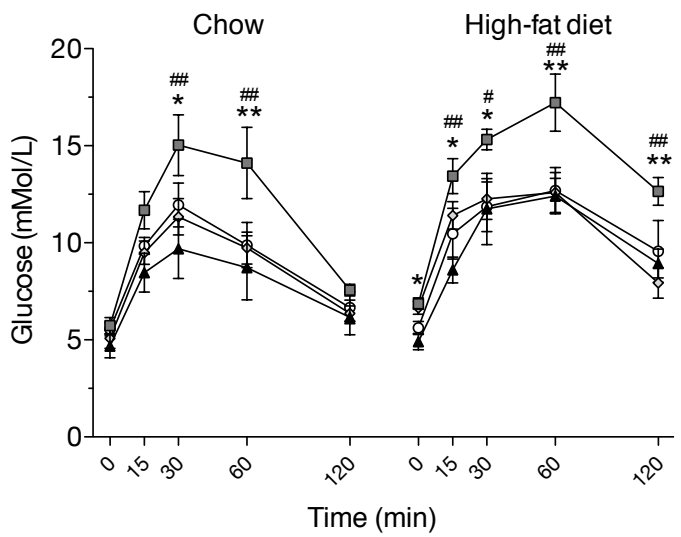

(b)

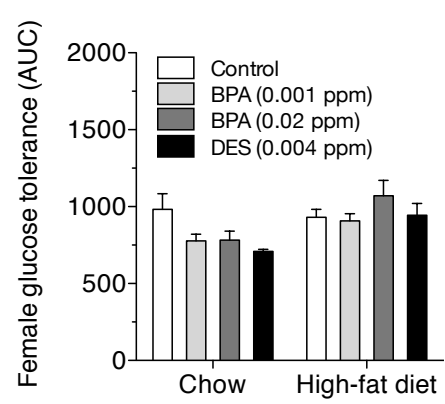

(d)

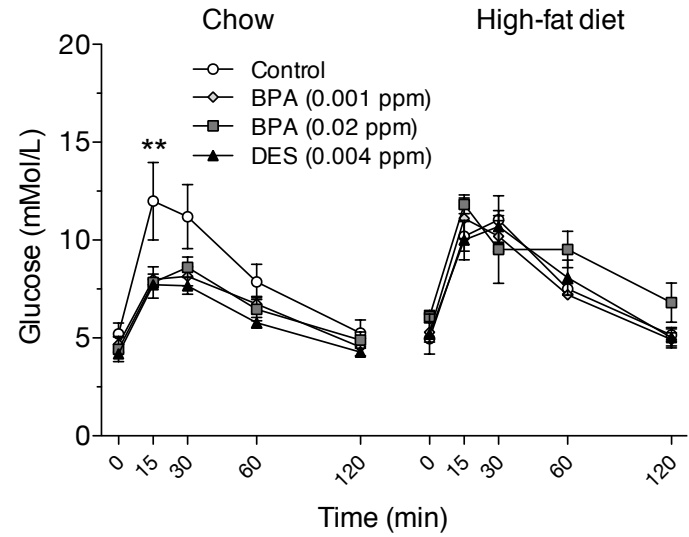

Figure 3.6: Glucose tolerance test.

Glucose tolerance test results expressed as area under the curve (AUC) in males (a) and females (b). Baseline and post-glucose injection blood glucose in chow- and high-fat diet-consuming males (c) and females (d). $* p<0.05, * * p<0.01$ vs. control animals of the same sex and group; $\# p<0.05$, $\# \# p<0.01$ vs. DES-treated animals of the same sex and group; $\mathbf{t \dagger} p<0.01$ vs. animals of the same sex and group consuming chow. All values expressed as mean \pm SEM. 
(a)

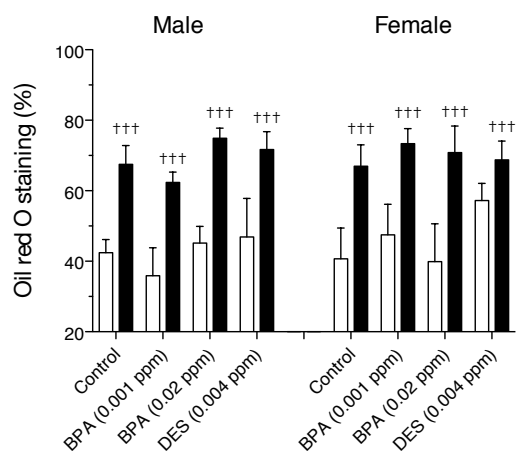

(b)

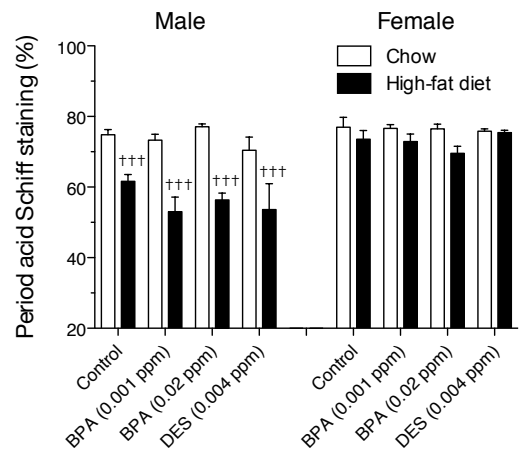

Figure 3.7: Hepatic Oil Red $\mathbf{O}$ and periodic acid-Schiff staining

Results from histological analysis of hepatic lipid and glycogen content. Oil Red O staining (a), periodic acid-Schiff staining (b). $\mathbf{t + t} p<0.001$ vs. animals of the same sex and group consuming chow. All values expressed as mean \pm SEM. 


\begin{tabular}{|c|c|c|c|c|c|c|c|c|c|}
\hline & & \multicolumn{4}{|c|}{ Male } & \multicolumn{4}{|c|}{ Female } \\
\hline & & Control & $\begin{array}{l}\text { BPA } \\
(0.001 \mathrm{ppm})\end{array}$ & $\begin{array}{l}\text { BPA } \\
(0.02 \mathrm{ppm})\end{array}$ & $\begin{array}{l}\text { DES } \\
(0.004 \mathrm{ppm})\end{array}$ & Control & $\begin{array}{l}\text { BPA } \\
(0.001 \mathrm{ppm})\end{array}$ & $\begin{array}{l}\text { BPA } \\
(0.02 \mathrm{ppm})\end{array}$ & $\begin{array}{l}\text { DES } \\
(0.004 \mathrm{ppm})\end{array}$ \\
\hline \multirow[t]{2}{*}{ IL-6 (pg/ml) } & Chow & $3 \pm 2.15^{\mathrm{a}}$ & $1.25 \pm 0.39^{\mathrm{a}}$ & $1.63 \pm 0.48^{\mathrm{a}}$ & $2.46 \pm 1.24^{\mathrm{a}}$ & $1.92 \pm 1.76^{\mathrm{a}}$ & $1.04 \pm 0.33^{\mathrm{a}}$ & $3.93 \pm 2.01^{\mathrm{a}}$ & $1.4 \pm 1.24^{\mathrm{a}}$ \\
\hline & High-fat diet & $3.69 \pm 1.37^{\mathrm{a}}$ & $4.14 \pm 2.38^{\mathrm{a}}$ & $1.46 \pm 0.9^{\mathrm{a}}$ & $1.52 \pm 0.52^{\mathrm{a}}$ & $1.99 \pm 1.05^{\mathrm{a}}$ & $0.44 \pm 0.16^{\mathrm{a}}$ & $0.85 \pm 0.66^{\mathrm{a}}$ & $0.4 \pm 0.25^{\mathrm{a}}$ \\
\hline \multirow[t]{2}{*}{ Insulin (ng/ml) } & Chow & $8 \pm 1.03^{\mathrm{a}}$ & $18.4 \pm 5.73^{\mathrm{b}}$ & $10 \pm 2.47^{\mathrm{a}}$ & $4.97 \pm 1.98^{\mathrm{a}}$ & $2 \pm 0.48^{\mathrm{a}}$ & $2.79 \pm 0.81^{\mathrm{a}}$ & $1.99 \pm 0.64^{\mathrm{a}}$ & $6.54 \pm 4.28^{a}$ \\
\hline & High-fat diet & $15.94 \pm 3.87^{\mathrm{a}}$ & $7.68 \pm 0.78^{c}$ & $34.58 \pm 3.13^{\mathrm{d}}$ & $30.25 \pm 8.31^{\mathrm{d}}$ & $2.17 \pm 0.4^{\mathrm{a}}$ & $2.88 \pm 0.3^{\mathrm{a}}$ & $3.44 \pm 0.21^{\mathrm{a}}$ & $1.33 \pm 0.43^{\mathrm{a}}$ \\
\hline \multirow[t]{2}{*}{ Resistin (ng/ml) } & Chow & $2.59 \pm 0.52^{\mathrm{a}}$ & $3.16 \pm 0.33^{\mathrm{a}}$ & $2.77 \pm 0.31^{\mathrm{a}}$ & $3.48 \pm 1.24^{\mathrm{a}}$ & $3.47 \pm 0.52^{\mathrm{a}}$ & $3.99 \pm 0.43^{\mathrm{a}}$ & $4.23 \pm 0.65^{\mathrm{a}}$ & $2.56 \pm 0.33^{\mathrm{a}}$ \\
\hline & Hight-fat diet & $3.04 \pm 0.17^{\mathrm{a}}$ & $6.2 \pm 1.78^{b}$ & $3.28 \pm 0.34^{\mathrm{a}}$ & $4.01 \pm 0.29^{\mathrm{a}}$ & $4.17 \pm 0.5^{\mathrm{a}}$ & $4.59 \pm 0.67^{\mathrm{a}}$ & $9.14 \pm 2.15^{\mathrm{b}}$ & $4.03 \pm 0.71^{\mathrm{a}}$ \\
\hline
\end{tabular}

Table 3.4: Circulating IL-6, insulin, and resistin.

Superscript letters indicate membership in homogenous subsets $(p<0.05)$. 
(a)

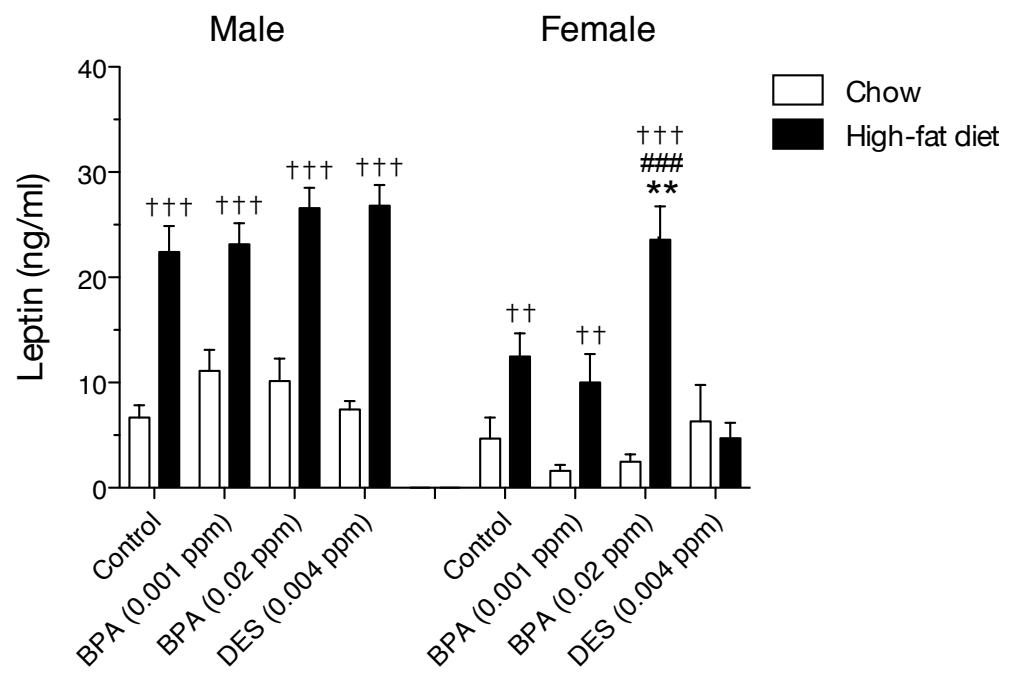

Figure 3.8: Circulating leptin in adult animals.

Leptin levels in plasma collected from trunk blood at sacrifice. $* * p<0.01$ vs. control animals of the same sex and group; \#\#\# $<0.001$ vs. DES-treated animals of the same sex and group; $\mathbf{t} \boldsymbol{t} p<0.01$, $\mathbf{t + t} p<0.001$ vs. animals of the same sex and group consuming chow. All values expressed as mean \pm SEM. 
(a)

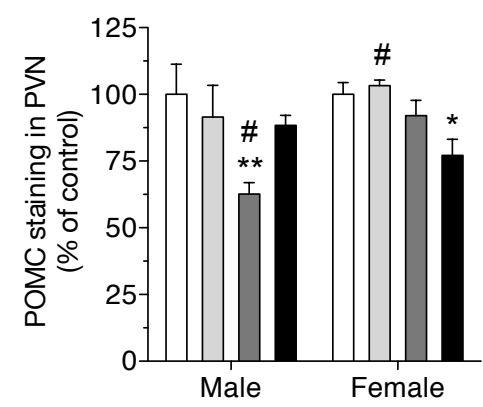

(d)

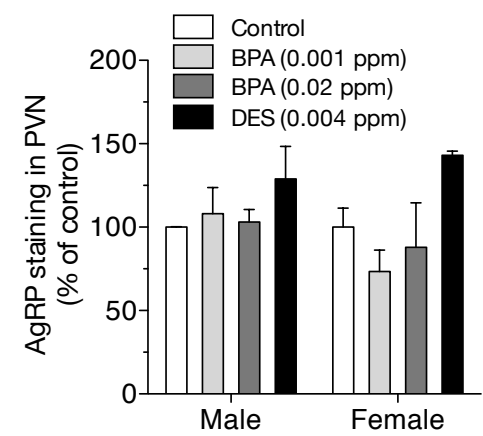

(b) Control male

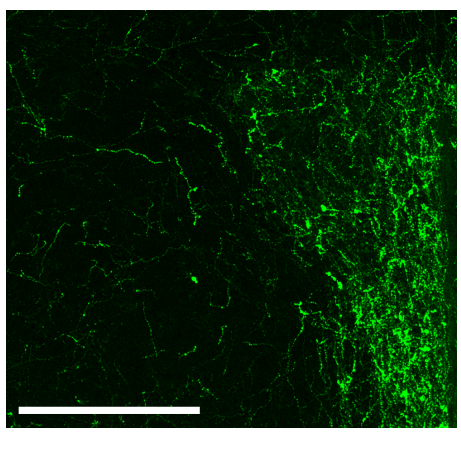

(e) Control female

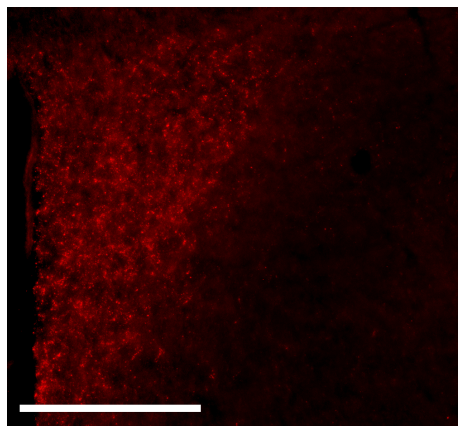

(c) BPA (0.02 ppm) male

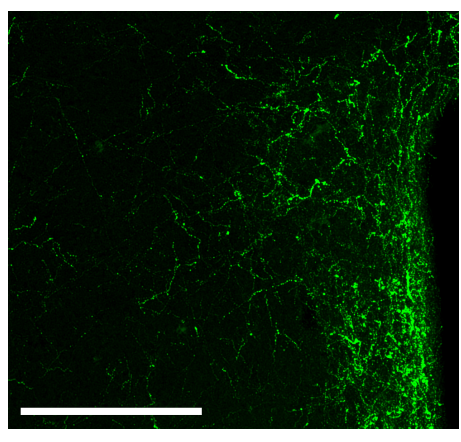

(f) DES (0.004 ppm) female

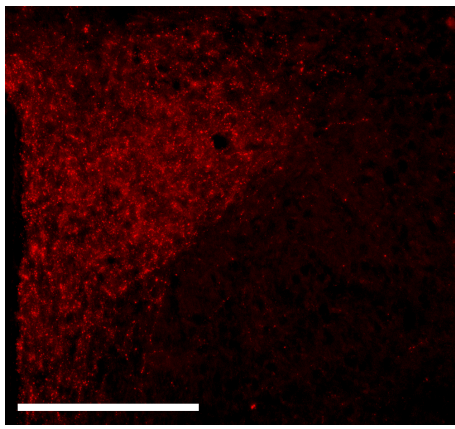

Figure 3.9: Immunofluorescence analysis of hypothalamic structure.

POMC fibre density in the PVN (a), Control male (b), 0.02 ppm BPA-treated male (c); AgRP fibre density in the PVN (d), Control female (e), DES-treated female (f). Images captured at 10x magnification, scale bars $=250 \mu \mathrm{m} . * p<0.05, * * p<0.01$ vs. control animals of the same sex and group; $\# p<0.05$, $\# \# p<0.01$ vs. DES-treated animals of the same sex and group. All values expressed as mean \pm SEM. Continued... 

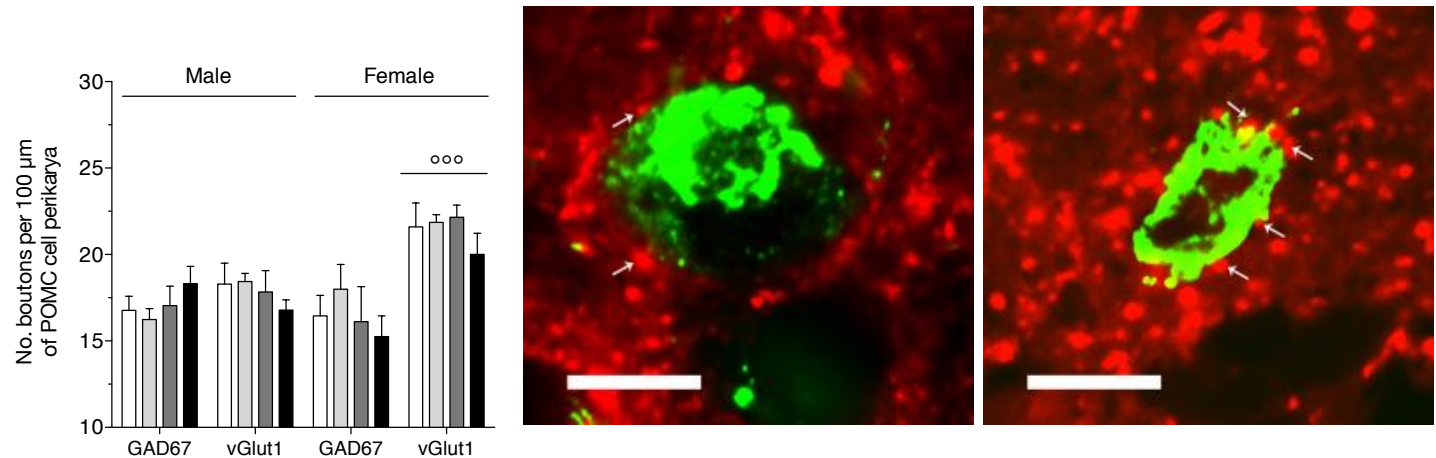

Figure 3.9: Continued. Immunofluorescence analysis of hypothalamic structure.

Number of putative excitatory vGlut1 and inhibitory GAD67 boutons in contact with POMC cells per $100 \mu \mathrm{m}$ perikarya (g), representative vGlut1 (red) and POMC (green) staining in Control male (h), Control female (j). Images captured at 100x magnification, scale bars $=10 \mu \mathrm{m} . \circ \circ \circ p<0.001$ vs. opposite sex animals from the same treatment. All values expressed as mean \pm SEM. 
(a)

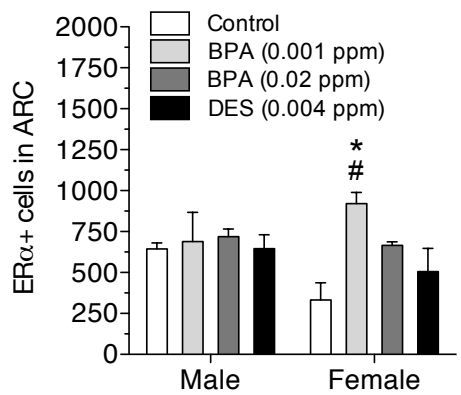

(d) Control female

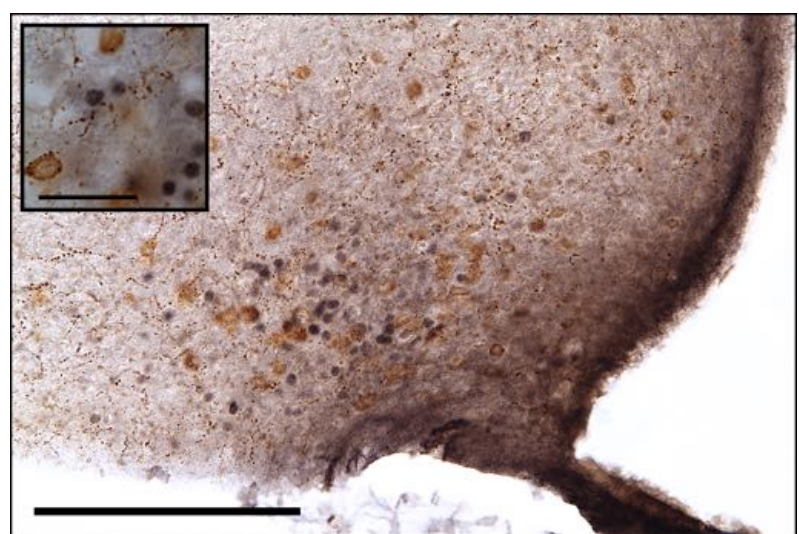

(c)
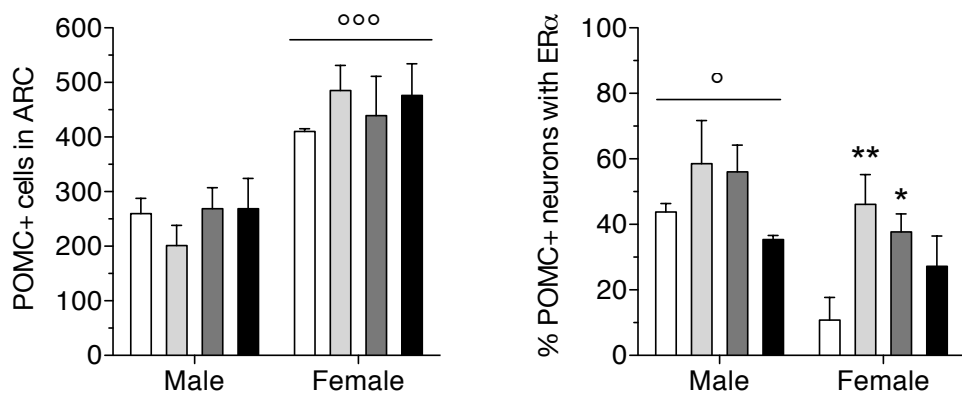

(e) BPA (0.002 ppm) female

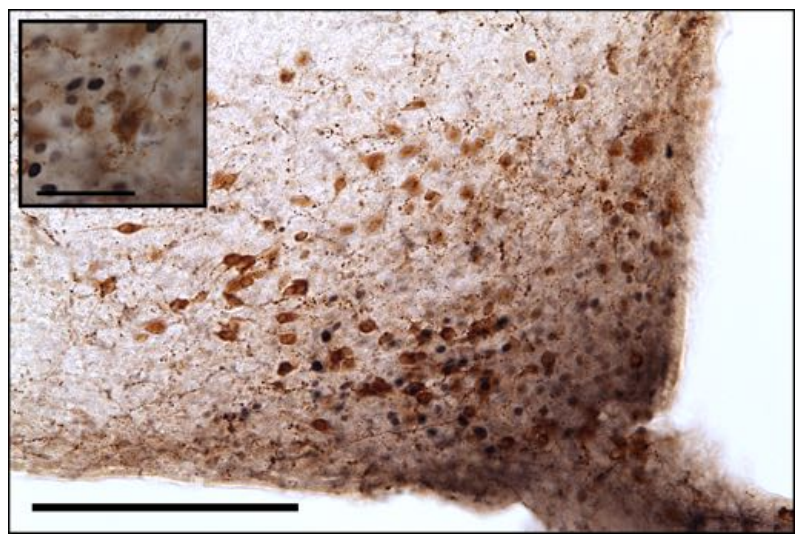

Figure 3.10: ER $\alpha$ and POMC immunohistochemistry.

ER $\alpha$ immunopositive nuclei (a), POMC immunopositive neurons (b), and the $\%$ of POMC cells that coexpress ER $\alpha$ (c) as revealed by immunohistochemistry. Representative images of female Control (d) and $0.02 \mathrm{ppm}$ BPA-treated (e) slices. ER $\alpha$ is revealed as blue nuclear staining, POMC as brown cytoplasmic staining. Images captured at $20 \mathrm{x}$ magnification, scale bars $=200 \mu \mathrm{m}$. Insets captured at $60 \mathrm{x}$ magnification, scale bars $=50 \mu \mathrm{m} . * p<0.05, * * p<0.01$ vs. control animals of the same sex; $\# p<0.05$ vs. DES-treated animals of the same sex and group; o $p<0.05$, $\circ \circ \circ p<0.001$ vs. opposite sex animals from the same treatment. All values expressed as mean $\pm \mathrm{SEM}$. 
(a)

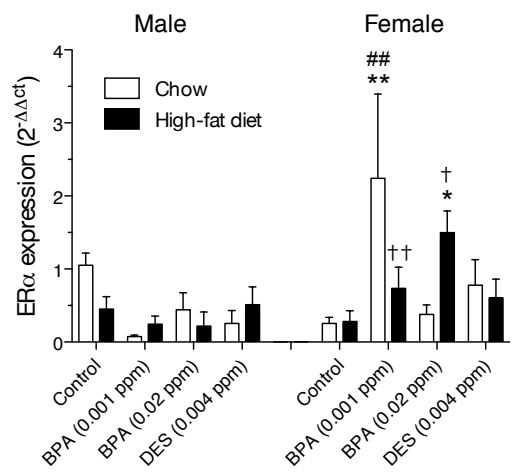

(c)

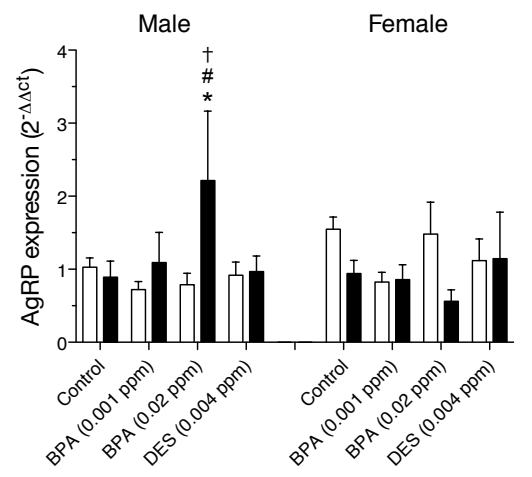

(b)

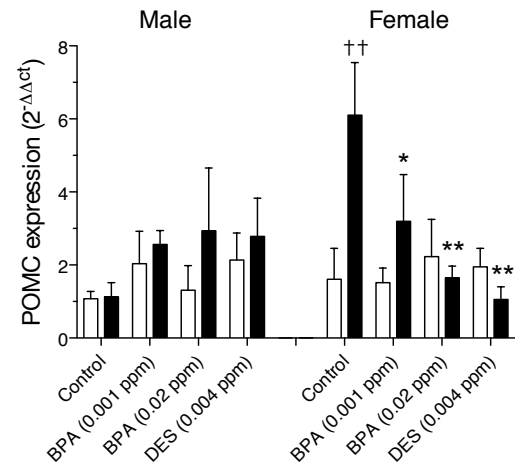

(d)

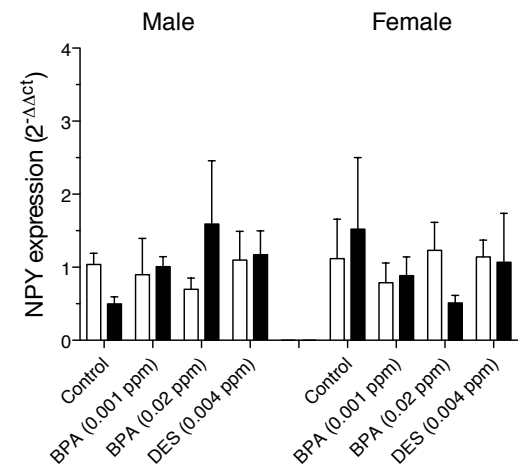

Figure 3.11: Gene expression in the arcuate nucleus.

$\mathrm{ER} \alpha$ (a), POMC (b), AgRP (c) and NPY (d) expression in the ARC in adult animals consuming either chow or the high-fat diet. $* p<0.05, * * p<0.01$ vs. control animals of the same sex and group; $\# p<0.05$, \#\#p $<0.01$ vs. DES-treated animals of the same sex and group; $\mathbf{\dagger} p<0.05, \mathbf{\dagger}+p<0.01$ vs. animals of the same sex and group consuming chow. All values expressed as mean \pm SEM. Continued... 
(e)

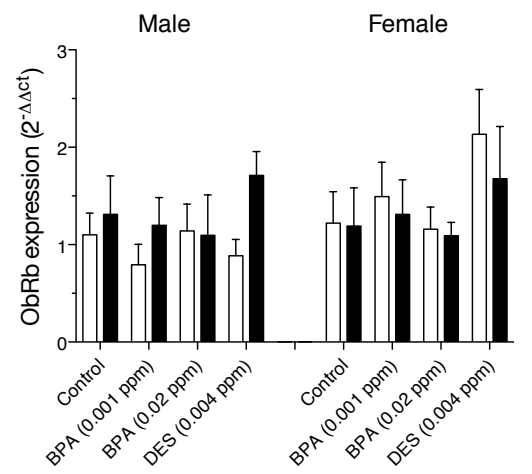

(g)

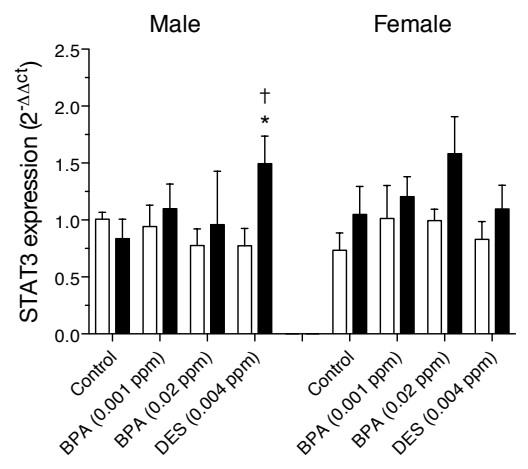

(f)

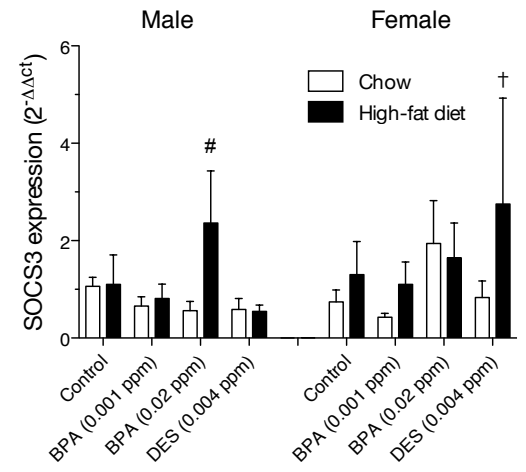

(h)

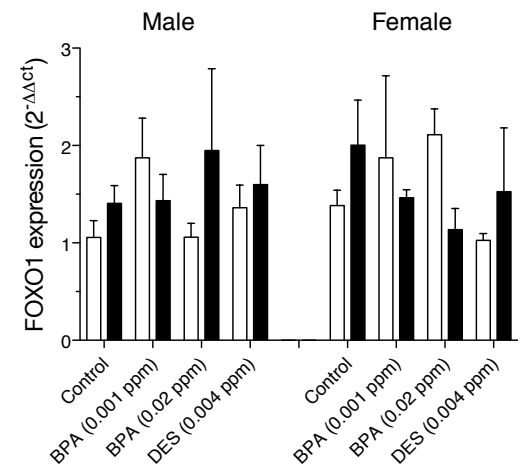

Figure 3.11: Continued. Gene expression in the arcuate nucleus.

ObRb (a), SOCS3 (b), STAT3 (c) and FOXO1 (d) expression in the ARC in adult animals consuming either chow or the high-fat diet. $* p<0.05$ vs. control animals of the same sex and group; \# $p<0.05$ vs. DES-treated animals of the same sex and group; $\mathbf{\dagger} p<0.05$ vs. animals of the same sex and group consuming chow. All values expressed as mean \pm SEM. 


\section{Chapter 4}

\section{Perinatal Exposure to Low-Dose Bisphenol-A and Impaired Central and Behavioural Leptin Sensitivity in Adulthood}

\subsection{INTRODUCTION}

Bisphenol-A (BPA) is a compound used extensively in the production of polycarbonate plastics, epoxy resins, and the consumer products made from these plastics [4]. Because BPA can leach from plastic containers into the food contained within, humans can be exposed to small doses of BPA whenever they consume food or drink that has been stored in these containers $[4 ; 6 ; 7]$ (see Section 1.1). BPA is a well-described endocrine disrupting compound (EDC), having been shown to be active on classical and membrane-bound estrogen receptors (see Section 1.3.1), as well as receptors for a variety of other steroid hormones (see Section 1.3).

Emerging evidence suggests that early-life BPA exposure is obesogenic, predisposing rodents to hyperphagia, obesity, and metabolic dysfunction in adulthood (see Section 1.5). Perinatal exposure to doses of BPA as low as $50 \mu \mathrm{g} / \mathrm{kg} /$ day, equivalent to the US EPA reference dose, is obesogenic in both male and female rats, and this effect is amplified by postnatal exposure to a high-fat diet [144]. Similarly, we have recently shown that perinatal exposure to BPA at an even lower average dose $(4.2-10.79 \mu \mathrm{g} / \mathrm{kg} /$ day, see Table 3.3) is obesogenic specifically in females exposed to a high-fat diet in adulthood [292]. Male offspring exposed to the same dose do not show a tendency to obesity in excess of Control males, but they do exhibit impaired glucose tolerance on both a control and high-fat diet [292]. This finding has been reported a number of times, almost always occurring exclusively in male animals [140; 292; 141; 144].

In our previous study (see Chapter 3), we developed a model of oral exposure to BPA at two low, environmentally-relevant doses, as well as a dose of diethylstilbestrol (DES) used as a positive control for estrogenic effects. Using this model, we 
showed that perinatal exposure to BPA at $0.02 \mathrm{ppm}$ produced a sex-specific phenotype of obesity-proneness in females, and impaired glucose tolerance in males, both of which can be uncovered by adult exposure to a high-fat diet. We noted that BPAexposed females consuming the high-fat diet were obese and hyperleptinemic, yet remained hyperphagic and failed to show any sign of POMC up-regulation in the ARC, and this may be taken as circumstantial evidence of leptin resistance. Leptin resistance can develop as a consequence of obesity, often through leptin receptor-dependent up-regulation of SOCS3, and the subsequent breakdown of pSTAT3 signalling in hypothalamic feeding nuclei $[177 ; 178]$. But leptin resistance can also be programmed by the early-life environment, and thus exist in a sort of latent form until made apparent by challenge with exogenous leptin or diet-induced obesity (see Section 2.3).

Perinatal BPA exposure has been shown in several studies to alter the regularity of the adult estrous cycle [136; 293; 294], leading in some cases to a state of permanent estrus. The precise mechanism of this effect has not yet been determined, though a decrease in estrogen sensitivity is a possibility. Since food intake varies across the estrus cycle in female rodents (see Section 2.4.1), it may be the case that BPA's sex-specific obesogenic effect is due to a breakdown in the estrogenic control of feeding. This could be due to a complete lack of a functioning estrous cycle in our BPA-exposed females, or it could simply be that food intake remains constant across the cycle, and that the usual drop in feeding associated with proestrus is absent.

In this study, we explored the possibility that animals exposed to our obesogenic model of environmental BPA exposure exhibit deficits in the behavioural and neurobiological response to leptin in advance of diet-induced obesity. Additionally, we explored the dynamics of feeding across the estrous cycle in female animals in order to estimate the effect, if any, that BPA has on the estrogenic regulation of feeding.

\subsection{METHODS}

\subsubsection{Animals}

All procedures were approved by the Carleton University Animal Care Committee and followed the guidelines of the Canadian Council on Animal Care. Animals used in this study were littermates of those sampled in Chapter 5. Virgin female CD-1 mice $(N=32)$ were purchased from Charles River and housed in polysulfone cages with ad-lib access to purified AIN93G control diet (Research Diets) and tap water in glass bottles. Animals were maintained on a $12 \mathrm{~h}$ light/dark cycle (lights on at 8:00). Female mice were weight-matched and assigned into treatment groups prior to mating. These females were housed in pairs along with a male mouse. AIN93G was used as the control diet, as well as the basis for the three treatment diets. BPA or DES $(>99 \%$ pure, Sigma Aldrich) was added to AIN93G by incorporation with the oil component 
of these diets (see Table A.1 for details on diet composition). Mated females were inspected every morning for evidence of a vaginal plug, the presence of which was taken to mark gestational day $0(\mathrm{G} 0)$. Confirmed pregnant females were single-housed and placed immediately on one of three specially prepared diets: (1) AIN93G control diet (Control), (2) AIN93G with $20 \mu \mathrm{g} / \mathrm{kg} \operatorname{diet~BPA~(0.02~ppm~BPA),~(3)~AIN93G~with~}$ $4 \mu \mathrm{g} / \mathrm{kg}$ diet diethylstilbestrol (0.004 ppm DES). DES is used here as a positive control for the estrogenic effects of BPA [159; 150]. Food intake and body weight of dams was recorded on alternating days in order to estimate exposure to the experimental compounds. Litters were standardized to 10 pups each on P2, with equal sex ratios where possible. On P21, pups were weaned, single-housed, and maintained thereafter on the Control diet.

(a) Study design

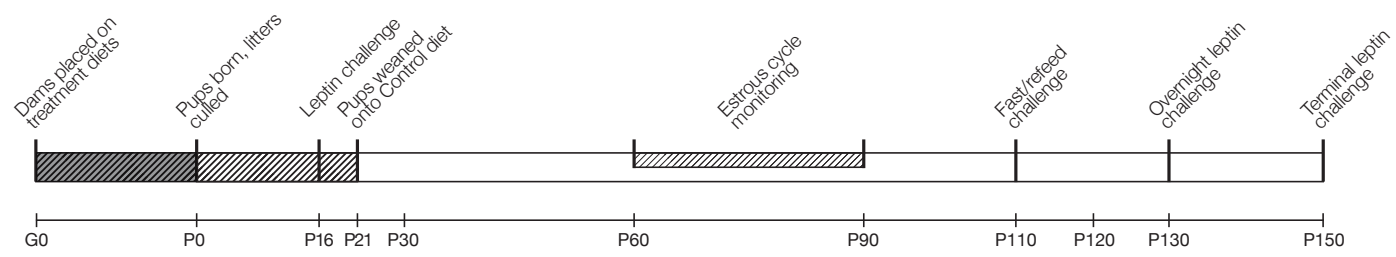

\subsubsection{Leptin Challenge at P16}

At P16, a subset of pups in Cohort 1 received an intraperitoneal injection of recombinant mouse leptin (PeproTech) at $3 \mu \mathrm{g} / \mathrm{g}$ and were sacrificed 45 minutes later by decapitation. Brains were rapidly removed and immersion fixed in $2 \%$ paraformaldehyde for $24 \mathrm{~h}$ at $4{ }^{\circ} \mathrm{C}$. Following fixation, brains were cryoprotected in $30 \%$ sucrose at $4{ }^{\circ} \mathrm{C}$. Brains were then cryosectioned at $40 \mu \mathrm{m}$ in one-in-four series, and sections were stored in Watson's cryoprotectant at $-20^{\circ} \mathrm{C}$ until used for pSTAT3 immunofluorescent staining.

\subsubsection{PSTAT3 Immunofluorescence}

Brain slices from Cohort 1 pups challenged with leptin at P16 were washed in PBS, then placed in a solution containing $1 \% \mathrm{NaOH}$ and $1 \% \mathrm{H}_{2} \mathrm{O}_{2}$ for 20 minutes. Following this, sections were washed and placed in a solution containing $0.3 \%$ glycine for 
10 minutes. Sections were washed again and incubated for 10 minutes in a solution containing $0.03 \%$ sodium dodecyl sulphate. After these pre-treatment steps, sections were blocked at room temperature for one hour in a solution of PBS containing 1\% bovine serum albumen (BSA), 1\% normal goat serum (NGS), and $0.3 \%$ Triton-X 1000 . The same blocking solution was used as the diluent in the primary antibody solution, which contained rabbit anti-pSTAT3 (Tyr705) antibody (1:500 Cell Signalling Technology). Sections were incubated in the primary antibody solution for $24 \mathrm{~h}$ at $4{ }^{\circ} \mathrm{C}$, washed, then incubated in a solution containing donkey anti-rabbit Alexa Fluor 488 secondary antibody (1:200, Life Technologies). Sections were then mounted and coverslipped with Prolong Gold mounting medium with DAPI counterstaining.

\subsubsection{Quantification of PSTAT3 Immunopositive Nuclei}

Images from immunofluorescently labelled sections collected at P16 were captured at 10x magnification using an Olympus BX61 microscope. Bilateral photomicrographs of the ARC, VMH, LH and DMH were collected through sections encompassing the rostral and caudal extent of the hypothalamus (3-5 sections per nucleus, depending on its size). Labelled nuclei were counted manually using ImageJ (National Institutes of Health) using regions of interest defined by the DAPI counterstained images.

\subsubsection{Estrous Cycle Monitoring}

The estrus cycle was monitored by vaginal lavage in post-pubertal female offspring from Cohort 1 beginning at P60. Smears were collected in the morning (9:00-10:00). Vaginal smears were placed on glass slides and allowed to air dry. Smears were fixed by brief immersion in 100\% methanol, followed by a brief wash in $\mathrm{dH}_{2} \mathrm{O}$. Slides were then stained for one minute in $0.1 \%$ crystal violet (Sigma) in $\mathrm{dH}_{2} \mathrm{O}$, followed by a one minute rinse in $\mathrm{dH}_{2} \mathrm{O}$. Slides were air dried and analyzed by microscope. Estrous staging was accomplished by comparing the relative frequency of leukocytes, nucleated, and cornified epithelial cells as described in $[295 ; 296]$. Several measures were used to estimate the pattern of estrous cyclicity in females including a count of the number of proestrous stages, the average number of days spent in estrous, and the average length of each cycle (defined as the number of days from one proestrous stage to the next). Food intake and body weight were measured daily in these females. The overnight food intake associated with each stage was pooled for every animal across the entire period of investigation (typically 5-6 full cycles) in order to explore the effect of the estrous cycle on food intake. 


\subsubsection{Fast/Refeed Challenge}

An overnight fast followed by a morning refeed was carried out in Cohort 1 animals at P110. Briefly, food was removed from animal cages at 19:00 and return the next morning at 9:00. Food intake was monitored hourly for six hours.

\subsubsection{Leptin Challenge}

At P130, Cohort 1 animals were given intraperitoneal saline injections at 19:00 for three days to establish a baseline measure of overnight food intake. After this, animals were given injections of recombinant mouse leptin $(2.5 \mu \mathrm{g} / \mathrm{g})$ at 19:00 for two nights, and overnight food intake and body weight change was monitored. Post-leptin overnight food intake was compared to baseline overnight food intake, and this was taken as an index of the behavioural sensitivity to leptin.

\subsubsection{Sacrifice and Tissue Preparation}

After a twenty day recovery period, Cohort 1 animals were fasted overnight and injected with leptin $(3.5 \mu \mathrm{g} / \mathrm{g})$ and sacrificed 6 hours later. Brains were rapidly removed and punches containing the mediobasal hypothalamus were removed and flash-frozen on dry ice for later analysis.

\subsection{9 qRT-PCR}

Micropunches of the mediobasal hypothalamus were homogenized, and RNA was isolated in $500 \mu \mathrm{l}$ TRIzol reagent (Life Technologies). Reverse-transcription was performed using the SuperScript II kit with oligo(DT) primer and the method provided by the manufacturer (Life Technologies). Real-Time PCR was performed in a MyiQ Single Colour Real-Time PCR Detection System (Bio-Rad) using iQ SYBR Green Super Mix (Bio-Rad). Gene expression was determined relative to the housekeeping control genes GAPDH and $\beta$-Actin using the $2^{-\Delta \Delta C t}$ method [273]. Saline Control males were used as the control group for all qRT-PCR analysis, thereby allowing for the detection of sexually dimorphic gene expression. All primer pairs were tested for efficiency over five orders of magnitude of template concentration. Primer sequences are listed in Table A.2.

\subsection{RESULTS}

\subsubsection{Effects of BPA and DES Treatment on Hypothalamic Leptin-Induced pSTAT3 at P16}

To determine whether BPA exposure acutely affects leptin sensitivity by interfering with signal transduction at the leptin receptor, we injected mice with leptin $(3 \mu \mathrm{g} / \mathrm{g}$, IP) 
at P16. These animals were sacrificed 45 minutes post-injection, and brains were processed for pSTAT3 immunofluorescence. We did not observe any sex- or treatmentrelated differences in the number of pSTAT3 immunopositive nuclei in the ARC, $\mathrm{VMH}$, or DMH $(\phi>0.05$; Figure 4.2). We did, however, observe a significant interaction between sex and treatment in the number of pSTAT3 labelled nuclei in the LH $\left(F_{(2,21)}=6.37, p<0.01 ;\right.$ Figure 4.2f). Post-hoc analysis indicated that DES-exposed males had significantly more pSTAT3 labelled nuclei than their Controls $(p<0.05)$, while both BPA- and DES-exposed females had significantly fewer PSTAT3 labelled nuclei than their Controls ( $\phi<0.05$ and $p<0.01$ respectively). Additionally, Control females had significantly more pSTAT3 labelled nuclei than male Controls $(p<0.05)$.

\subsubsection{Estrous Cyclicity is Unaffected by BPA or DES Treatment}

Both BPA and DES have previously been suggested to affect female reproductive function $[136 ; 293 ; 294 ; 156 ; 155]$. We were particularly interested in the possibility that early-life BPA treatment would disrupt the estrous cycle in adulthood, and in so doing attenuate the activational effects of circulating estradiol on feeding. To begin to explore this prospect, we employed a relatively simple approach of monitoring estrous cycles for 30 days in adult females. While there were occasional periods of extended diestrous in females from the Control group and each of the treatment groups, we did not observe any consistent deficit in estrous cyclicity. Over the 30 day period there were no treatment-related differences in the average number of estrous cycles, the average number of days spent in the estrous stage, or the average period of the cycle $(p>0.05$; Figure 4.3).

We monitored food intake across the estrous cycle, typically using data from 4-7 cycles per animal, depending on the frequency and reliability with which that animal cycled. Data from periods of extended diestrus or estrus were excluded from the analysis. Food intake typically drops on the night of proestrus but this pattern is absent, for example, in animals lacking functioning AgRP neurons [241], and several other manipulations besides (see Section 2.4.1). We were interested in whether this pattern would be present in our BPA-exposed females, knowing that they tended to become obese and hyperphagic when challenged appropriately. We used repeated measures ANOVA to analyze feeding across the pooled stages of the estrous cycle. This analysis showed a main effect of stage, highlighting the expected drop in feeding associated with the estrus stage $\left(F_{(2.41,50.69)}=7.23, p<0.001\right.$ by Greenhouse-Geisser correction; Figure 4.4). However, we found no significant interaction between stage and maternal treatment, suggesting that the ovarian control of food intake remains largely unaltered in BPA- and DES-exposed females. 


\subsubsection{Rebound Feeding is Greater in Females, but not Affected by BPA or DES Treatment}

The NPY/AgRP neuron population in the ARC plays a critical role in mediating rebound feeding following an overnight fast [297]. Exogenous estradiol, likely through its actions on these neurons, attenuates the refeeding response in female animals [298; 241]. In contrast, if the fast/refeed paradigm is carried out during diestrus female rats show an exaggerated refeed response [247]. The origins of this effect are not known, but given that it is a fairly robust sex difference in feeding behaviour we decided to investigate it in our BPA-exposed animals. We reasoned that if this sex difference were ablated or even reversed, it could point to a defect in the post-ingestive feedback control over feeding. Food intake adjusted to body weight was significantly higher in females during the first hour of refeeding $\left(F_{(1,43)}=41.153, p<0.001\right.$; Figure 4.5a), and again in the period between the $4^{\text {th }}$ and $6^{\text {th }}$ hours of refeeding $\left(F_{(1,43)}=7.869, p<\right.$ 0.01; Figure 4.5a). Cumulative food intake over the entire six hour period of measurement was significantly higher in females than in males $\left(F_{(1,43)}=33.343, p<0.001\right.$; Figure 4.5b). We did not detect any main effects of maternal treatment, nor any interactions between that and sex $(p>0.05)$.

\subsubsection{Attenuated Leptin Sensitivity in BPA- and DES-Exposed Animals}

To test the possibility that BPA-exposed mice are leptin resistant in advance of dietinduced obesity, we conducted an overnight leptin sensitivity test in adult animals. Because baseline body weight was not affected by maternal treatment (Figure 4.6), we analyzed leptin-induced overnight weight loss in absolute terms. Leptin administration lead to an overnight decline in body weight in each of the groups, though this effect was significantly attenuated in BPA- and DES-exposed animals $\left(F_{(1,42)}=4.103, p<0.05\right.$; $p<0.05$ for BPA and DES vs. Control animals by planned comparisons; Figure 4.7a). Though we did identify a small decrease in overnight food intake, this did not differ significantly between the various maternal treatments ( $p>0.05$; Figure 4.7b).

Having previously shown that neither BPA nor DES treatment affect leptin-induced pSTAT3 labelling at P16, we decided to move further downstream in our study of adult leptin sensitivity. To this end, we examined leptin-induced changes in gene transcription in the mediobasal hypothalamus $6 \mathrm{~h}$ post-injection. We found that POMC mRNA was significantly upregulated by leptin injection in both males and female Control animals, but not in BPA- or DES-exposed animals $(p<0.05$; Figure 4.8a). We found that SOCS3 mRNA was significantly upregulated by leptin in BPA treated females only $(p<0.05$; Figure 4.8d). We did not detect a difference in AgRP or NPY mRNA in response either to maternal treatment or leptin injection. 


\subsection{DISCUSSION}

Our previous studies (see Chapter 3) left us with the impression that animals exposed perinatally to BPA at $0.02 \mathrm{ppm}$ might have been exhibiting programmed leptin resistance that was revealed under the metabolic challenge of a high-fat diet. On the basis of those results alone, however, that hypothesis could not be verified. In this series of studies, we examined leptin sensitivity in animals raised to adulthood under identical conditions. Our data show that animals exposed perinatally to the endocrine disrupting compounds BPA and DES show, even in the absence of an obese phenotype, deficiencies in their response to exogenous leptin treatment. These findings provide the first evidence that endocrine disrupting compounds given at environmentally relevant doses can program adult leptin sensitivity in a manner analogous to the well-documented nutritional and genetic models of developmental programming.

We were initially curious as to whether exposure to BPA or DES interfered with leptin receptor signalling via its JAK/STAT3 effectors. This form of leptin receptor signalling can be disrupted either by prolonged hyperleptinemia, arising as a consequence of diet-induced obesity [177; 178], and by developmentally programmed obesity [200]. ARC neurons express leptin receptors at relatively low levels at P6 and earlier but these levels increase markedly by P10 [299]. As leptin receptor expression becomes more abundant in the developing hypothalamus, central sensitivity to leptin increases in lockstep [299; 300; 301]. Two reasons lead us to examine leptin sensitivity at this timepoint. Firstly, animals at this time point are exposed to the experimental compounds both through their bourgeoning consumption of solid food and also through their continuing reliance on milk, and testing leptin sensitivity at this timepoint could address whether BPA or DES acutely inhibit leptin sensitivity ${ }^{1}$. Secondly, central leptin insensitivity has been noted to appear as early as P16 in models of early-life overnutrition [200]. With no estrous cycle to control for, limited occasion for environmental variability or stress, and limited influence from any other of life's vicissitudes, animals tested at this timepoint are theoretically less hampered by confounding factors. Thus a difference, if found, may be more closely linked to compound exposure itself and not some other intervening factor. Ultimately the only nucleus showing any sex or treatment effects was the $\mathrm{LH}$, and here we found that Control females had significantly greater levels of leptin-induced pSTAT3 than Control males, but this difference was absent in females exposed BPA and DES (Figure 4.2f). The orexin and melaninconcentrating hormone $(\mathrm{MCH})$ neural populations inhabit the $\mathrm{LH}$, and the orexin system in particular shows several notable sexual dimorphisms. Females express higher

\footnotetext{
${ }^{1}$ In fact, if a difference in leptin sensitivity had been detected, this difference would have necessitated a followup study in compound-exposed P16 animals that had been switched to the control diet prior to the leptin challenge to determine whether the altered sensitivity was programmed or simply an acute response to circulating BPA or DES.
} 
levels of orexin mRNA, their orexin neurons respond more vigorously to fasting, and females show a much stronger body weight phenotype in response to orexin knockout than do males $[247 ; 302 ; 303]$. Neither orexin nor MCH neurons express the leptin receptor $\mathrm{ObRb}$ themselves, rather their activity is responsive to leptin through indirect means, that is by the inhibitory influence of nearby ObRb-expressing neurotensin neurons $[304 ; 305 ; 306]$. There may be a hitherto unidentified sex difference in these first order neurons that can be modified or reversed by xenoestrogen treatment, but such a prospect requires further study.

Neonatally masculinized females present an obesity-prone phenotype in adulthood, and this appears to depend on developmentally programmed changes in the hypothalamic melanocortin system [238]. Nohara et al., noted that their neonatally masculinized females showed the overt signs of programmed leptin resistance-namely a diminished sensitivity to its anorectic effects in-vivo [238]. We reasoned that BPA, acting the part of a sex steroid mimetic, might produce similar results in our animals. Indeed, we found that both BPA and DES exposure lead to a programmed attenuation of leptin's effect on body weight. Regardless of sex, animals exposed to these compounds lost less weight following overnight leptin treatment than Control animals, though the effect certainly appeared to be the strongest in BPA-exposed females (Figure 4.7a). Curiously, neonatal masculinization does not produce leptin resistance in the brain through the usual channel of reduced pSTAT3 signal transduction, rather the effect is seen further downstream, at the level of POMC transcription [238]. We found a similar outcome in our animals, with no apparent difference in leptin-induced pSTAT3 in the mediobasal nuclei of the hypothalamus, but a notable absence of leptin-induced POMC upregulation (Figure 4.8a). Our data on SOCS3 transcription, which shows the expected transcriptional increase in leptin-treated females, suggest that the basic link between leptin receptor stimulation and the activation of gene transcription is not at fault, at least in females $[172 ; 178 ; 173]$. Rather it is POMC upregulation that is specifically affected by xenoestrogen exposure, and while the reason for this remains uncertain, epigenetic programming of the POMC promoter is an attractive hypothesis (see Section 2.5). This phenotype was concordant between BPA- and DES-exposed animals, and while the pharmacological activities of BPA are more diverse than those of the estrogenic positive control DES, the most parsimonious explanation for any observed overlap involves an estrogenic mechanism.

Since our model of BPA exposure is obesogenic in females only, one interpretation of the data is that BPA eliminated the sex differences that normally protect females from diet-induced obesity (see Chapter 3). A number of subtle sex differences exist between male and female patterns of eating in rodents (see Section 2.4.2), and if any of these were absent in xenoestrogen-exposed animals, then the case could be made for altered sexual differentiation. We chose to conduct a fast/refeed test to explore this possibility, because there is a documented sex difference in rebound feeding af- 
ter an overnight fast (though only when food consumption is measured relative to body weight) [247], because it varies according to the relative strength of orexigenic influences [297], and also because it could point to alterations in responsiveness to short-term post-ingestive signals. Though we found a robust sex difference in rebound feeding, we did not find any significant treatment-related effects, and this implies that the obesogenic effects of BPA do not likely depend on exaggerated orexigenic tone or impaired post-ingestive cues. Further study, of course, is needed to verify these inferences.

Cyclic production and central sensitivity to estradiol are crucial to the ability of female rodents to resist diet-induced obesity. If estradiol is withdrawn in adulthood by ovariectomy, for example, the hyperphagia and weight gain soon follow [209; 210; 217]. Similarly, if early-life conditions are arranged so as to masculinize the female brain, then the estrogenic regulation of feeding is disrupted in adulthood and obesity follows $[248 ; 249 ; 238]$. There is reason to believe that the xenoestrogens BPA and DES might act to masculinize perinatal female similarly to the usual standard of testosterone (aromatized to estradiol), for some reports have shown that early-life exposure to these compounds can disrupt the development of sexually dimorphic nuclei (see Section 1.4). A simple and non-invasive way of testing the effects of BPA or DES on the estrogenic regulation of feeding is to track daily food intake as a function of the estrous cycle. Ordinarily, female rodents show a marked decline in overnight food intake during the estrus phase of the estrous cycle [213]. BPA could disrupt or arrest the estrous cycle itself (e.g. [136]), and in that scenario there would of course be no cyclic variations in feeding. Alternatively, estrous cyclicity could remain intact, but daily food intake could simply remain constant over the usual estrus phase nadir. We tested both possibilities in this study, and we found no convincing evidence that either the estrous cycles themselves, or the estrous-related variations in food intake were substantially affected by xenoestrogen exposure. Both BPA- and DES-exposed females cycled with regularity that was comparable to Control animals, and all animals showed the expected decline in food intake at estrus (Figure 4.4).

Our aim in this series of studies was to explore the sensitivity of BPA- and DESexposed animals to metabolic hormones, in particular the potently anorectic leptin and estradiol, through the use of behavioural and neurobiological approaches. In testing both, we found little to suggest that impaired responsiveness to estradiol could account for the hyperphagic phenotype of BPA-exposed females, but a great deal in favour of programmed leptin insensitivity. The latter can arise as a consequence of early-life estrogenic stimulation, as seen in [238], and since BPA and DES can act in an estrogenic capacity (see Section 1.3.1) there is good reason to suspect a mechanistic overlap between the two processes. However this alone cannot account for everything. Female BPA-exposed mice do not, in our hands, acquire male-like rebound feeding patterns, nor is the programmed leptin insensitivity they demonstrate even a part of the typical 
male phenotype. For that matter, male animals seem to develop programmed leptin insensitivity to a near-equal extent as females when exposed to our test compounds. It does not seem accurate, then, to argue that BPA is obesogenic because is masculinizes the brain, even if that theory is the easiest to support with our current understanding of BPA's receptor and enzyme affinities (see Section 1.3). BPA and DES may affect some other organ, and its secretions or neurological connections may themselves affect the development of the brain's metabolic circuitry in a non-sex-specific manner, but this and other suppositions require additional research. 
(a)

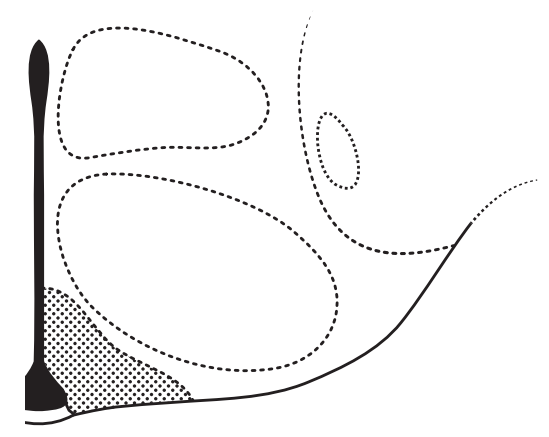

(c) Control male

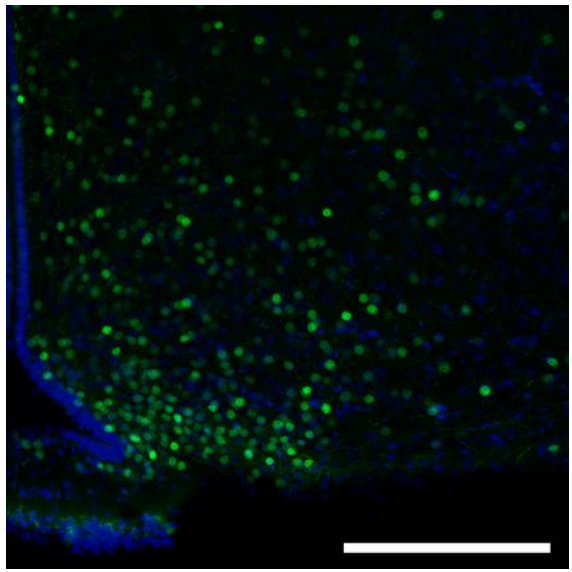

(b)

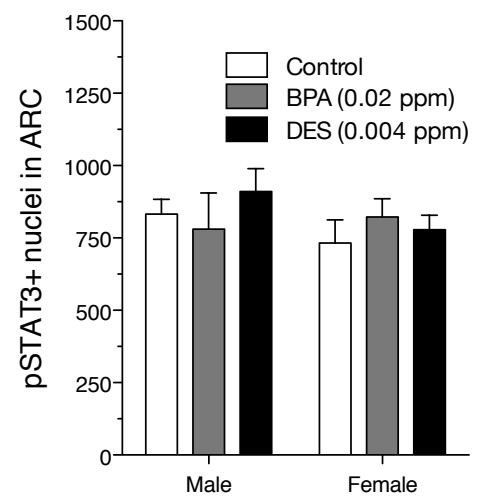

(d) BPA (0.02 ppm) male

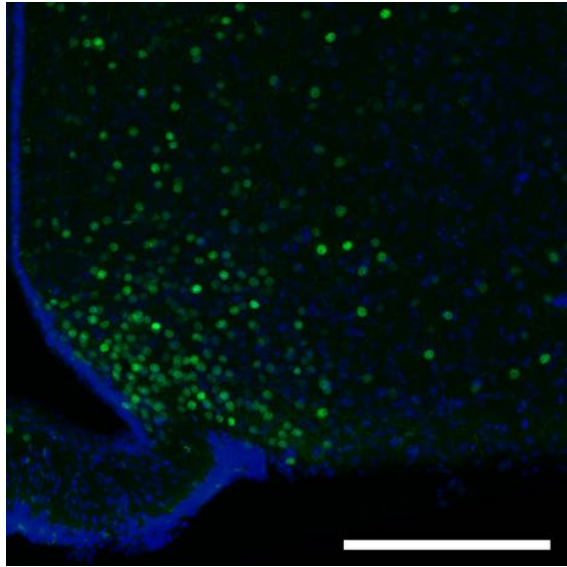

Figure 4.2: Leptin-induced pSTAT3 immunofluorescence at P16.

pSTAT3 labeled cells in the ARC (b), male Control (c), male BPA (d). All photomicrographs captured at 10x magnification with DAPI nuclear counterstaining, scale bars $=250 \mu \mathrm{m}$. All values expressed as mean \pm SEM. Continued... 
(e)

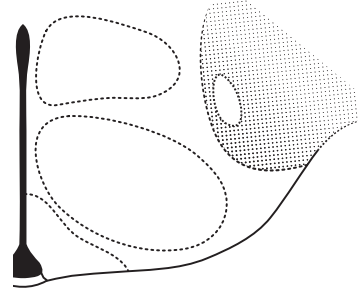

(i)

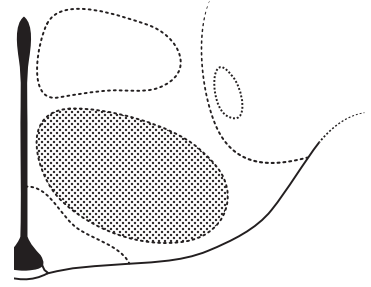

(m)

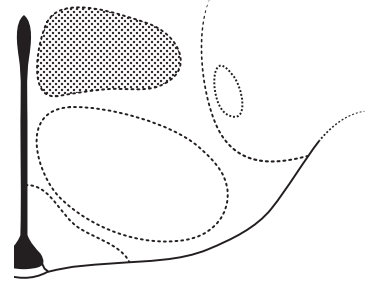

(f)

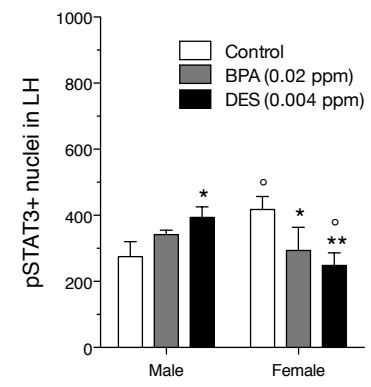

(j)

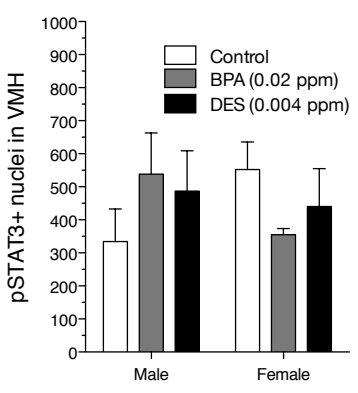

(n)

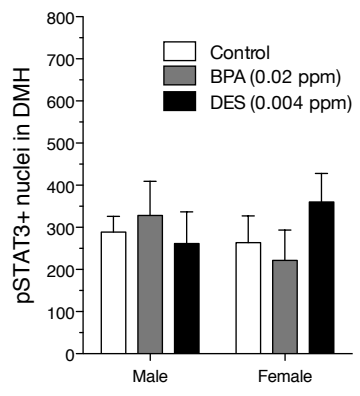

(g) Control female

(h) BPA (0.02 ppm) female
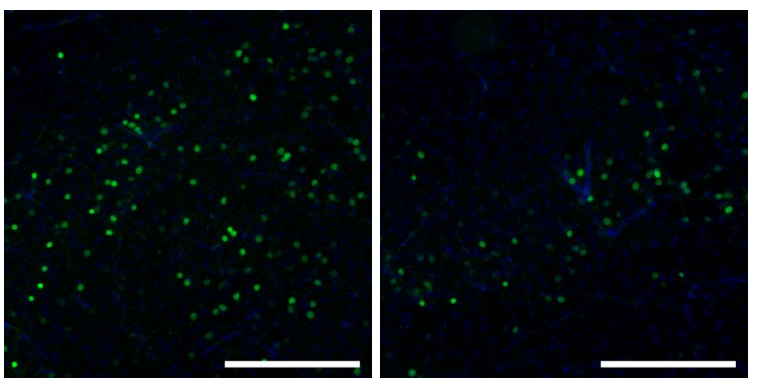

(k) Control female

(l) BPA (0.02 ppm) female
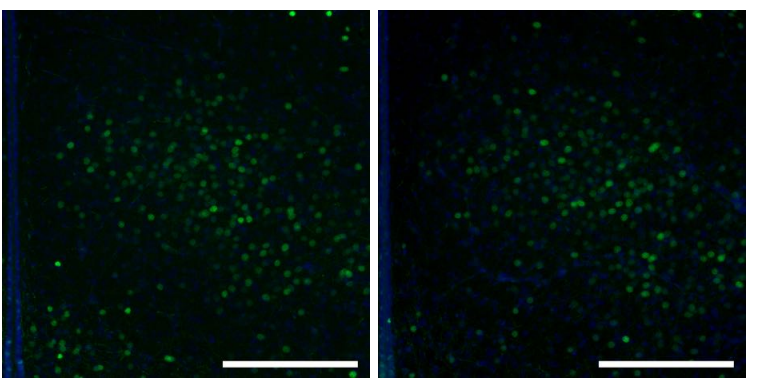

(o) Control male

(p) BPA (0.02 ppm) male

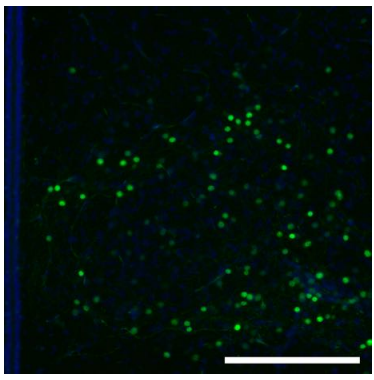

Figure 4.2: Continued. Leptin-induced pSTAT3 immunofluorescence at P16.

pSTAT3 labeled cells in LH (f); female Control (g), BPA (h). VMH (j); female Control (k), BPA (l). DMH (n); male Control (o), BPA (p). $* p<0.05, * * p<0.01$ vs. Control animals of the same sex and group; $\circ p<0.05$ vs. male animals from the same group. All photomicrographs captured at 10x magnification with DAPI nuclear counterstaining, scale bars $=250 \mu \mathrm{m}$. All values expressed as mean $\pm \mathrm{SEM}$. 
(a)

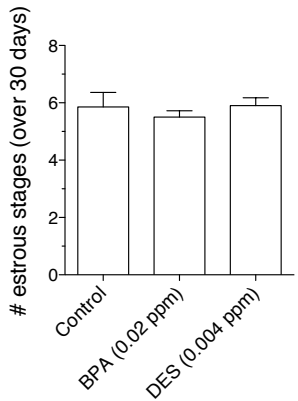

(d) Control

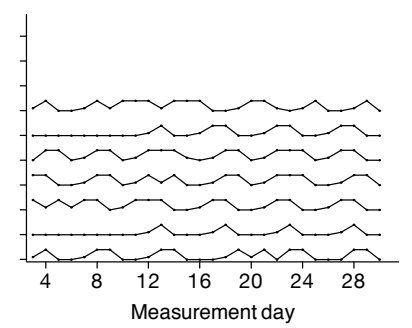

(b)

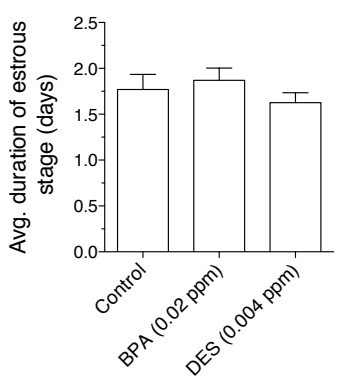

(e) BPA (0.02 ppm)

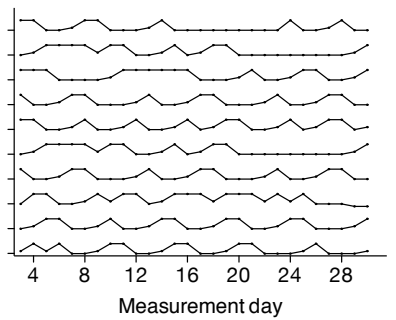

(c)

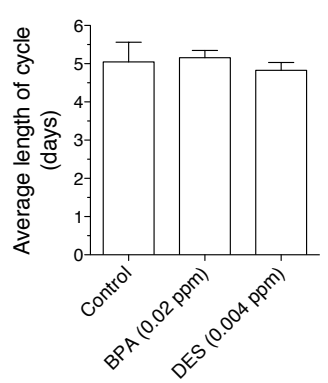

(f) DES (0.004 ppm)

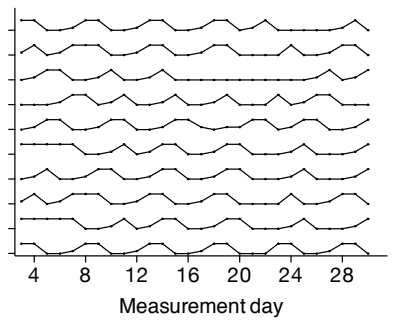

(g) Proestrus

(h) Estrus

(i) Diestus I

(j) Diestrus II

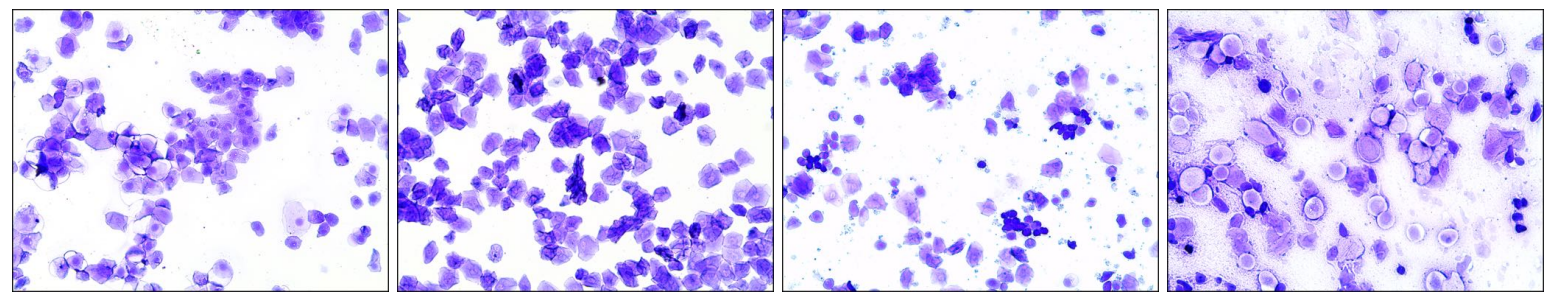

Figure 4.3: Estrous cycle. Adult estrous cycles over 30 days of observation. Number of entries into the estrous stage of the cycle (a). Average number of days spent in each estrus phase (b). Average length of cycle (c). Traces of estrous cycles in Control (d), BPA (e) and DES (f) treated females. Example of crystal violet-stained vaginal smear from animal in proestrus (g), estrus (h), diestrus I (i), and diestrus II (j). Peaks represent estrus phases and troughs represent diestrus phases. All values expressed as mean \pm SEM. 
(a)

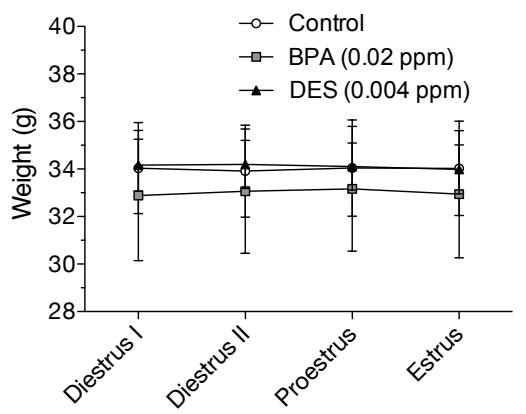

(b)

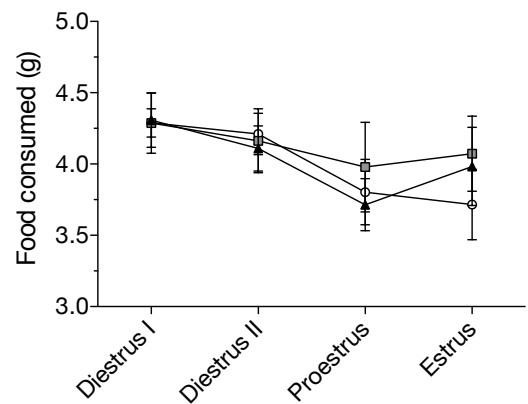

Figure 4.4: Food intake over the estrous cycle.

Adult female body weight averaged across diestrus I, diestrus II, proestrus and estrus (a). Food intake averaged across diestrus I, diestrus II, proestrus, and estrus (b). All values expressed as mean \pm SEM.

(a)

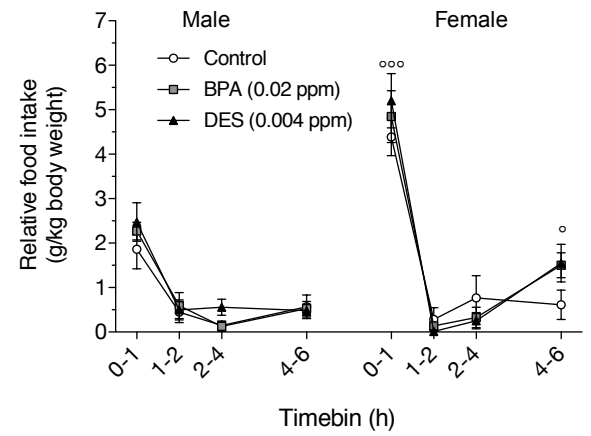

(b)

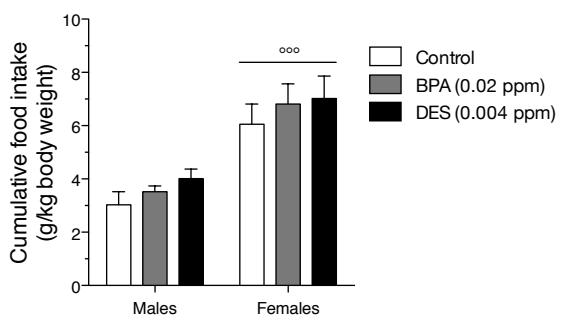

Figure 4.5: Overnight fast/refeed challenge.

Food intake as a proportion of body weight in the first 6 hours of refeeding (a). Cumulative food intake over the first 6 hours of refeeding (b). $\circ \circ \circ p<0.001$ vs. opposite sex animals. All values expressed as mean \pm SEM. 


\begin{tabular}{lllll}
\hline & & Control & BPA $(0.02 \mathrm{ppm})$ & DES $(0.004 \mathrm{ppm})$ \\
\hline Male & Body weight $(\mathrm{g})$ & $46.52 \pm 2.55$ & $49.72 \pm 2.99$ & $49.46 \pm 2.18$ \\
& Food intake $(\mathrm{g})$ & $3.04 \pm 0.13$ & $3.58 \pm 0.19$ & $3.45 \pm 0.24$ \\
\hline Female & Body weight $(\mathrm{g})$ & $36.28 \pm 1.97$ & $37.67 \pm 3.61$ & $37.03 \pm 1.32$ \\
& Food intake $(\mathrm{g})$ & $3.10 \pm 0.18$ & $3.22 \pm 0.19$ & $3.31 \pm 0.18$ \\
\hline
\end{tabular}

Figure 4.6: Baseline body weight and average nightly food intake

There were no significant differences in body weight or food intake between any groups. All values expressed as mean \pm SEM.

(a)

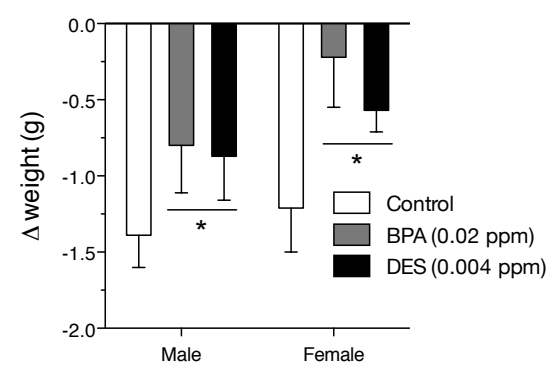

(b)

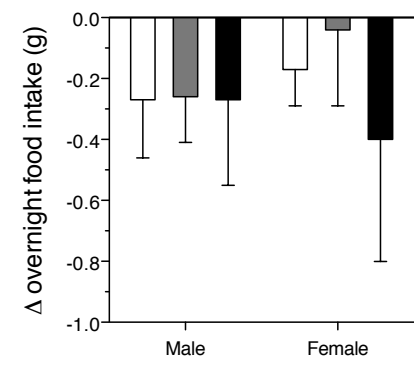

Figure 4.7: Metabolic response to leptin.

Body weight loss from saline baseline after two nights of leptin $(2.5 \mu \mathrm{g} / \mathrm{g}$ IP) (a). Average leptin-induced reduction in overnight food intake from saline baseline over two nights (b). $* p<0.05$ vs. Control animals. All values expressed as mean \pm SEM. 
(a)

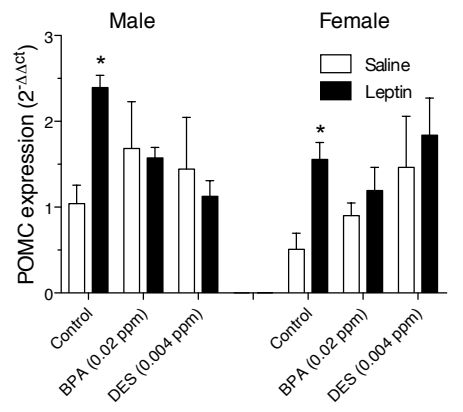

(c)

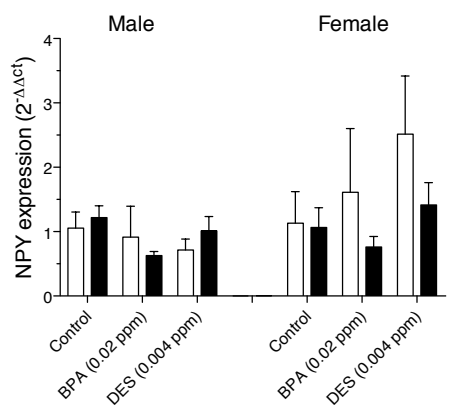

(b)

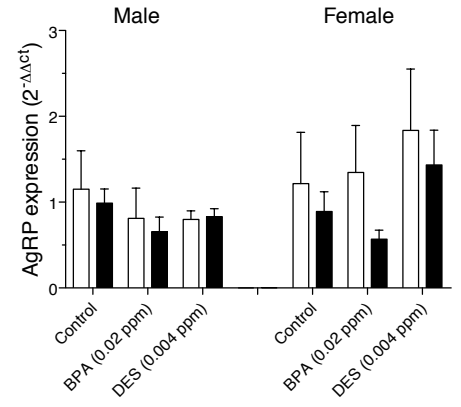

(d)

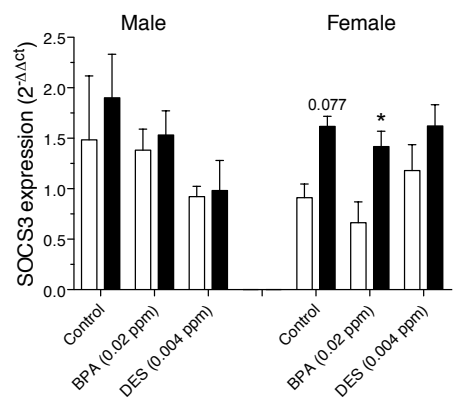

Figure 4.8: Leptin-induced gene expression in mediobasal hypothalamus.

POMC expression (a), AgRP (b), NPY (c), SOCS3 (d). $* p<0.05$ vs. saline injected animals of same sex and group. All values expressed as mean \pm SEM. 


\section{Chapter 5}

\section{Perinatal Exposure to Low-Dose Bisphenol-A (BPA) Alters the Postnatal Leptin Surge Leading to Impaired Development of Hypothalamic Feed- ing Circuits}

\subsection{INTRODUCTION}

Bisphenol-A (BPA) is a chemical plasticizer that is used widely in the production of polycarbonate plastics and epoxy resins, and found extensively in food and drink containers made from these compounds [4]. Under certain conditions, most notably those of high heat, alkalinity or acidity, or simply advanced age, BPA can leach from plastic containers into the food or drink they may contain $[4 ; 6 ; 7]$ (see Section 1.1). BPA is a well-described endocrine disrupting compound (EDC), having been shown to be active on classical and membrane-bound estrogen receptors (see Section 1.3.1), as well as receptors for a variety of other steroid hormones (see Section 1.3).

In Chapters 3 and 4 we demonstrated that early-life exposure to BPA induces longlasting changes in the structure and function of the hypothalamic feeding circuitry. One component of this phenotype is a form of programmed leptin resistance, first inferred in Chapter 3 and later verified in Chapter 4. The better part of the brain's leptin-responsive neurons are born on E12 [307], and it is during this time period that the developing brain is especially susceptible to nutritional influences that affect neurogenesis and, subsequently, the adult function of affected nuclei. Prenatal overand undernutrition affect the rate of embryonic neurogenesis, respectively resulting in hyper- and hypocellularity in hypothalamic feeding nuclei, and in both cases yielding dysfunctions in feeding and metabolism $[308 ; 309 ; 191]$. BPA too can affect neurogenesis, as can be inferred by its ability to inhibit the proliferation of hippocampal-derived neural stem cells [310]. It is not known at present whether BPA inhibits neurogenesis in the mammalian hypothalamus, but if it does, this could account for some measure 
of its programming effects.

In Chapter 3 we also noted that BPA-exposed male animals showed reduced innervation of the PVN by POMC-containing axonal fibres. This phenomenon was first described by Bouret et al., who showed that these projections are almost completely absent in leptin deficient ob/ob mice, but can be rescued by perinatal leptin supplementation [286]. Subsequent investigations have substantially elaborated upon these findings, showing that perinatal undernutrition [206; 253], overnutrition [311], maternal diabetes [312], and genetically-predisposed obesity [202] can all impair the growth of these fibres. While there are a number of models of developmental programming, each presumably with its own unique characteristics, there does seem to be a central role for perinatal leptin in nearly all of the models examined thus far. Though adipose tissue makes up a relatively small proportion of the animal's body mass early in life, there is nonetheless a marked surge in circulating leptin in the first 10 days of life [185]. This leptin surge does not influence food intake, but it does appear to be critical in the development of leptin-sensitive neural circuitry [185]. If the leptin surge is upset, as it is in studies of maternal food restriction [206; 207] and obesity [197], long-term functional and structural changes in the hypothalamic circuitry can result. It is perhaps for this reason that neonatal leptin supplementation is generally protective against obesity later in life $[313 ; 254 ; 314 ; 315 ; 316]$. Several in-vitro studies have shown that low doses of BPA can affect adipogenesis and leptin secretion in cultured adipocytes $[317 ; 117 ; 105]$. Given that perinatal leptin secretion is of critical importance for the programming of feeding circuitry, and that BPA can affect these processes, it may be the case that the obesogenic effects of BPA are mediated by leptin secreted by the adipose tissue early in life.

In this study, our goal was to explore the early-life neuromorphological correlates of the programming effects we have previously uncovered. Since prenatal neurogenesis and postnatal wiring are two factors with the clearest link to other forms of developmental programming, we hypothesized that these two processes would be impaired by BPA exposure. Owing to the importance of leptin's neurotrophic effects in mediating the postnatal development of this circuitry and the ability of BPA to alter leptin secretion, we also hypothesized that the postnatal leptin surge would be impaired in BPA-exposed animals and that exogenous leptin supplementation could rescue the neurodevelopmental phenotype.

\subsection{METHODS}

\subsubsection{Animals}

All procedures were approved by the Carleton University Animal Care Committee and followed the guidelines of the Canadian Council on Animal Care. Virgin female CD-1 
mice ( $N=32$ for Cohort $1, N=15$ for Cohort 2) were purchased from Charles River and housed in polysulfone cages with ad-libitum access to purified AIN93G control diet (Research Diets) and tap water in glass bottles. Animals were maintained on a $12 \mathrm{~h}$ light/dark cycle (lights on at 8:00). Female mice were weight-matched and assigned into treatment groups prior to mating. These females were housed in pairs along with a male mouse. AIN93G was used as the control diet, as well as the basis for the three treatment diets. BPA or DES ( $>99 \%$ pure, Sigma Aldrich) was added to AIN93G by incorporation with the oil component of these diets (see Table A.1 for details on diet composition). Mated females were inspected every morning for evidence of a vaginal plug, the presence of which was taken to mark gestational day 0 (G0). Confirmed pregnant females were single-housed and placed immediately on one of three specially prepared diets: (1) AIN93G control diet (Control), (2) AIN93G with $20 \mu \mathrm{g} / \mathrm{kg}$ diet BPA (0.02 ppm BPA), (3) AIN93G with $4 \mu \mathrm{g} / \mathrm{kg}$ diet diethylstilbestrol (0.004 ppm DES). DES is used here as a positive control for the estrogenic effects of BPA [159; 150]. Food intake and body weight of dams was recorded on alternating days in order to estimate exposure to the experimental compounds. Litters were standardized to 10 pups each at birth, with equal sex ratios where possible. Litters born with fewer than 8 , or more than 12 pups were excluded from analysis. 
(a) Study design for Cohort 1

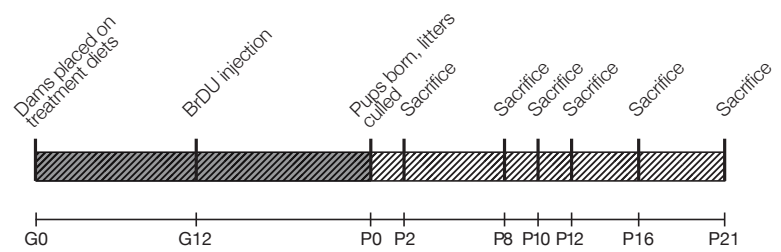

(b) Study design for Cohort 2

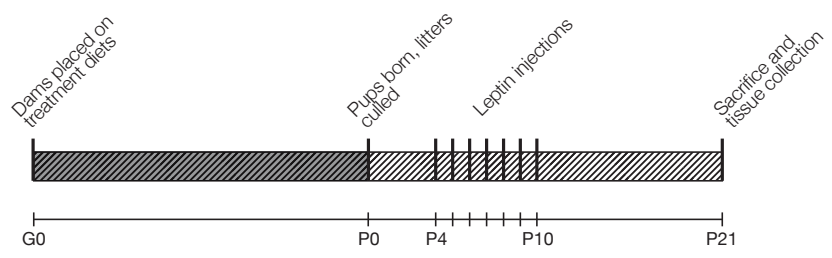

\subsubsection{BrDU Injection}

At approximately 11:00 on E12 confirmed pregnant dams in Cohort 1 were injected with 5-bromo- 2 -deoxyuridine (BrDU, Sigma) in $0.9 \%$ saline with $0.007 \mathrm{~N} \mathrm{NaOH}$ at $160 \mathrm{mg} / \mathrm{kg}$. A bolus injection of BrDU at this timepoint has been confirmed to capture proliferation of prospective leptin-responsive neurons in the mouse [307]. Correct timing of the injection was verified by backdating from the litter's birth date, knowing that CD-1 mice give birth after 20 days of gestation. Litters that were injected on days other than E12 were removed from the analysis.

\subsubsection{Postnatal Leptin Supplementation}

Male and female pups from Cohort 2 were given daily subcutaneous injections of either leptin $(5 \mu \mathrm{g} / \mathrm{g}$ in $0.9 \%$ saline with $0.001 \mathrm{M} \mathrm{NaOH})$ or the saline vehicle from P4 until P10. Each litter contributed two pups of each sex, with one receiving saline and the other leptin, yielding $N=60$ for this cohort. Body weight was measured daily in these animals during the period of injection. Since our model of BPA and DES exposure does not produce any changes in body weight during the early postnatal period (see Figure 5.2), we expressed the body weight of leptin-treated animals as a percent of their saline controls to better visualize the effect of leptin on body weight. 


\subsubsection{Serum and Tissue Collection}

On postnatal days 2, 8, 10,12,16, and 21 pups from Cohort 1 were sacrificed by decapitation. Leptin- and saline-treated pups from Cohort 2 were sacrificed on P21. For Cohort 1, male and female pups were randomly selected from the pool of litters so that at any given timepoint, only one male and one female from each litter was analyzed. A pool of spare litters was maintained under identical conditions so that as pups were sampled from each of the test litters, replacement pups of the same age were crossfostered in as replacements in order to maintain a standardized litter size. Trunk blood samples were collected in $1.5 \mathrm{ml}$ microcentrifuge tubes pre-coated with EDTA, centrifuged to collect plasma. Plasma was stored at $-80^{\circ} \mathrm{C}$ until analysis. Inguinal white adipose tissue was also collected at each timepoint and stored at $-80^{\circ} \mathrm{C}$ until analysis. Brains collected at each timepoint were immersion fixed in $4 \%$ paraformaldehyde overnight at $4{ }^{\circ} \mathrm{C}$ before being transferred to $30 \%$ sucrose in advance of cryosectioning.

\subsubsection{Body Fat Determination}

Carcasses of saline- and leptin-treated animals from Cohort 2 sacrificed at P21 were frozen at $-20{ }^{\circ} \mathrm{C}$ prior to dissection. Perigonadal, retroperitoneal, subcutaneous fat pads, and interscapular brown adipose tissue (BAT) were dissected and weighed by an observer blind to treatment condition.

\subsubsection{Measurement of Circulating Leptin}

Serum leptin levels in samples collected from Cohort 1 were measured in duplicate using a mouse leptin ELISA kit (Millipore) according to the supplied directions. The sensitivity of this assay was $0.05 \mathrm{ng} / \mathrm{ml}$. The intra- and inter-assay coefficients of variation were $7.7 \%$ and $3.28 \%$ respectively. Because there was no effect of sex, nor any sex-related interactions, data from males and females were pooled for the final analysis.

\subsection{7 qRT-PCR Measurement of Leptin Expression}

Leptin mRNA was quantified in white adipose tissue samples collected from each pup in Cohort 1 at the time of sacrifice. Adipose tissue samples were homogenized in TRIzol reagent (Life Technologies). After homogenization, samples were centrifuged briefly, and the fatty supernatant was discarded. RNA quality was determined by measuring the 260/280 absorbance ratio and by agarose gel electrophoresis. Reverse transcription was performed using the SuperScript II kit with oligo(DT) primers and the method supplied by the manufacturer (Life Technologies). Real-time PCR was performed in a MyIQ Single Colour Real-Time PCR Detection System (Bio-Rad) using 
iQ SYBR Green Super Mix (Bio-Rad). The expression of leptin was determined relative to the housekeeping gene GAPDH (see Table A.2 for primer sequences) using the $2^{-\Delta \Delta C t}$ method [273]. Control animals at P2 were used as a baseline for analysis across postnatal timepoints.

\subsubsection{Immunofluorescence for POMC and BrDU}

Brains from Cohort 1 animals sacrificed at P16 and P21 were sliced at $40 \mu \mathrm{m}$ on a cryostat in a one-in-four series. BrDU incorporation was detected using immunofluorescent staining. Sections were washed in $0.1 \mathrm{M}$ phosphate buffer, then pre-treated in $1 \mathrm{~N} \mathrm{HCl}$ for 30 minutes at $45^{\circ} \mathrm{C}$ to denature the DNA and make $\mathrm{BrDU}$ residues accessible to the detection antibody. Sections were then blocked at room temperature for one hour in a solution of phosphate buffer containing $1 \%$ bovine serum albumen (BSA), 1\% normal goat serum (NGS), and 0.3\% Triton-X 1000. The primary antibody cocktails contained rat anti-BrDU (1:1000, AbCam) and rabbit anti-POMC (1:5000, Phoenix Pharmaceuticals; P21 only). Sections were incubated in the primary antibody cocktail overnight at room temperature, washed, then incubated in a secondary antibody cocktail containing donkey anti-rat Alexa Fluor 594 IgG and donkey anti-rabbit Alexa Fluor $488 \operatorname{IgG}$ (1:200, both from Life Technologies). Finally, sections were mounted on glass slides and coverslipped with Prolong Gold mounting medium with DAPI counterstaining.

\subsubsection{Immunofluorescence for POMC and AgRP}

Tissue sections from Cohort 2 animals were washed several times in $0.1 \mathrm{M} \mathrm{PB}$, then incubated in primary antibody cocktails contained rabbit anti-POMC $1: 2500$, Phoenix Pharmaceuticals) and goat anti-AgRP (1:500, Santa-Cruz Biotechnology) for 24 hours at room temperature under gentle agitation. The following day, sections were washed and then incubated for one hour in a cocktail consisting donkey anti-goat Alexa Fluor 594 conjugated IgG and donkey anti-rabbit Alexa Fluor 488 conjugated IgG (1:200, both from Life Technologies). Sections were mounted on glass slides and coverslipped using Prolong Gold containing 4',6-diamidino-2-phenylindole (DAPI) as a nuclear counterstain (Life Technologies).

\subsubsection{Quantification of BrDU Immunopositive Nuclei}

Images from immunofluorescently labelled sections collected from Cohort 1 animals at P16 were captured at 10x magnification using an Olympus BX61 microscope. Bilateral photomicrographs of the ARC, VMH, PVN, LH and DMH were collected through sections encompassing the rostral and caudal extent of the hypothalamus (3-5 sections per nucleus, depending on its size). Labelled nuclei were counted manually using 
ImageJ (National Institutes of Health) using regions of interest defined by the DAPI counterstained images.

\subsubsection{Quantification of POMC \& BrDU Co-Localization}

Sections collected from Cohort 1 animals at P21 immunofluorescently stained for POMC and BrDU were analyzed by confocal microscopy. Z-stacks were collected in the ARC under 20x magnification at $1 \mu \mathrm{m}$ intervals using an Olympus BX61 microscope equipped with an Olympus DSU disk-scanning confocal unit. POMC-, BrDU-, and double-labelled cells were manually counted through the Z-axis in the ARC along its rostrocaudal extent (6 sections).

\subsubsection{Quantification of POMC and AgRP Projections}

Stained sections from Cohort 2 were imaged an Olympus BX61 microscope equipped with an Olympus DSU disk-scanning confocal unit. DAPI counterstaining was used to locate the PVN, which was imaged bilaterally using Z-stacks at $1 \mu \mathrm{m}$ intervals at 10x magnification. Z-stacks were imaged using three filter sets (FITC, TRITC, and DAPI) allowing for the detecting of POMC, AgRP, and DAPI labelling in the same section. ImageJ (National Institutes of Health) was used to collapse Z-stacks into composite images. These composites were subsequently binarized and skeletonized using identical parameters for each image. The area fraction of POMC and AgRP immunolabelled fibres was quantified within the boundaries of the PVN established by the nuclear counterstain DAPI.

\subsubsection{Statistics}

To avoid confounds due to litter effects in Cohort 1 , at most one male and one female per litter was used at each timepoint. In Cohort 2, each litter contributed two animals of each sex which were assigned to receive either leptin or saline. Data are expressed in graphs as mean \pm SEM. Data were generally analyzed using two-way ANOVAs with sex and perinatal treatment as factors, except in the case of the postnatal leptin measures where data were pooled due to the lack of a discernible sex effect. Where applicable, leptin treatment was included as a factor, yielding a three-way ANOVA design. Significant interactions were explored by examining the simple main effects at each level of the interacting variable. Post-hoc tests were performed using Fisher's Protected LSD with a significance criterion set at $p<0.05$. Planned comparisons between BPA-treated animals and both Control and DES-treated animals of the same sex were conducted as a matter of course whenever justified by treatment effects or treatment by sex interactions. 


\subsection{RESULTS}

\subsubsection{Maternal and Pup Characteristics are not Affected by BPA or DES Treatment}

There were no effects of BPA or DES treatment on either maternal weight (see Figure 5.2a) or food intake (see Figure 5.2b) through pregnancy and lactation. Accordingly, the feed conversion rate was equivalent across treatment groups (see Figure 5.2c), indicating that neither treatment affected the efficiency with which dams converted calories into body mass. Litter size was not affected by either BPA or DES treatment (see Figure $5.2 \mathrm{~d}$ ), nor was there any effect of treatment on the ratio of male to female pups (see Figure 5.2e). Body weight did not differ between male and female offspring at any measured postnatal timepoint (see Figure 5.2f and 5.2g).

\subsubsection{Neurogenesis at E12 is not Affected by BPA or DES Treatment}

We used immunofluorescence to analyze BrDU incorporation in key hypothalamic feeding nuclei at P16. BrDU injected at G12 was widely incorporated into nuclei throughout the hypothalamus. Detailed inspection of $\mathrm{BrDU}$ incorporation in the ARC did not reveal any sex- or treatment-related differences $\left(F_{(1,21)}=0.17, p>0.05\right.$ and $F_{(2,21)}=0.16, p>0.05$ respectively; Figure 5.3b). Similarly, we found no sex- or treatment-related differences in $\mathrm{BrDU}$ immunopositive nuclei in the $\mathrm{VMH}(p>0.05$; Figure 5.3f), $\mathrm{LH}(\phi>0.05$; Figure 5.3j), DMH $(p>0.05$; Figure 5.3n), and PVN $(p>0.05$; Figure 5.3r).

\subsubsection{Embryonic Neurogenesis of POMC Neurons is not Affected by BPA or DES Treatment}

Using brains from animals sacrificed at P21, we examined the incorporation of $\mathrm{BrDU}$ injected maternally at G12 into POMC immunopositive neurons in the ARC. We did not find any sex- or treatment-related differences in POMC neuron count $(p>0.05$; Figure 5.4a), BrDU immunopositive nuclei count $(p>0.05$; Figure 5.4b), nor any effect on neurons co-labeled with POMC and $\mathrm{BrDU}(p>0.05$; Figure 5.4c). We also measured the intensity of POMC immunofluorescence by way of integrated density in order to obtain an estimate of POMC peptide expression. There were no significant sex- or treatment-related differences in integrated density ( $p>0.05$; Data not shown).

\subsubsection{Hypothalamic POMC Projections are Reduced in Males and Fe- males Exposed to BPA}

We used confocal microscopy to analyze the density of POMC immunopositive projections into the PVN in Cohort 1 animals sacrificed at P21. We found a significant 
main effect of $\operatorname{sex}\left(F_{(1,17)}=9.027, p<0.01\right.$; Figure $\left.5.5 \mathrm{~b}\right)$, as well as a significant main effect of maternal treatment $\left(F_{(2,17)}=4.402, p<0.05\right.$; Figure $\left.5.5 b\right)$. There was no interaction between sex and maternal treatment-females generally had higher levels of staining than males from the same treatment $(p<0.01)$. Analysis of the treatment effect by planned comparison revealed that BPA-exposed males and females had significant reductions in POMC projections relative to Control animals $(p<0.05$, Figure $5.5 b)$.

\subsubsection{BPA and DES Disrupt the Postnatal Leptin Surge}

Our initial analysis of leptin concentration failed to reveal a main effect of $\operatorname{sex}\left(F_{(1,75)}=\right.$ $0.190, p>0.05)$ or an interaction between sex and treatment $\left(F_{(1,75)}=0.807, p>\right.$ $0.05)$, in agreement with reports that the leptin surge is identical in male and female pups [318]. As such, we carried out further analyses with data collapsed across sex. We found significant main effects of time $\left(F_{(5,93)}=8.38, p<0.001\right)$, treatment $\left(F_{(2,93)}=\right.$ $4.37, p<0.05)$, and a significant interaction between treatment and time $\left(F_{(10,93)}=\right.$ $4.23, p<0.001$; Figure 5.6a). Leptin concentration showed the expected spike at P8 in Control animals, but this was notably absent in BPA- and DES-treated animals $(p<0.01$ vs. Control at P8). Leptin concentration was elevated in BPA-treated animals at $\mathrm{P} 10$ ( $p<0.05$ vs. Control, $p<0.01$ vs. DES) and P12 $(p<0.01$ vs. Control, $p<0.01$ vs. DES), suggestive of a retarded leptin surge. DES-treated animals failed to show any spike in leptin concentration, with levels peaking at $\mathrm{P} 16(\phi<0.001$ vs. DES at P2, $p>0.5$ vs. Control and BPA animals at P16). To test the role of leptin gene transcription in modulating circulating leptin levels, we analyzed leptin mRNA in white adipose tissue using qRT-PCR. We found that leptin mRNA in white adipose tissue showed the same basic trend, though there were no significant main effects of either treatment or time, nor a significant interaction between the two $(\phi>0.05$; Figure $5.6 b)$.

\subsubsection{Body Weight and Composition is not Affected by Postnatal Leptin Treatment}

Absolute body weight in saline- and leptin-treated animals from Cohort 2 did not differ among maternal treatments at any time (data not shown). To better visualize the effect of leptin treatment on body weight, given the absence of pre-existing differences, we expressed the body weights of leptin-treated animals as a percent of the average body weight of saline-injected animals of the same sex and maternal treatment. Neither this approach, nor absolute body weight revealed any effect of leptin injection on growth or body weight (Figure 5.7). Fat pad weight was assessed in Cohort 2 animals sacrificed at P21. Here we found significant main effects of sex in each of the measured fat pads $\left(p<0.001\right.$; Figure 5.8). We also found a significant main effect of treatment $\left(F_{(1,49)}=\right.$ 
$5.312, p<0.05)$ in subcutaneous fat pad weight, attributable to DES-exposed animals $(p<0.05$ by linear contrast; Figure 5.8). Fat pad weight was not influenced by leptin injection in any case.

\subsubsection{Postnatal Leptin Treatment Rescues Impaired POMC Projections in Females Exposed to BPA}

To test whether our observation of reduced POMC projections into the PVN in Cohort 1 animals could be attributed to an altered pattern of postnatal leptin secretion, we studied the same pathway in animals from Cohort 2 that had been given supplemental leptin during the surge period. In these animals, we observed a significant interaction between sex and injection $\left(F_{(1,47)}=6.548, p<0.05\right.$; Figure 5.9a). Upon exploring the simple main effects in this interaction, we found a significant effect of maternal treatment among saline-injected animals $\left(F_{(2,23)}=3.471, p<0.05\right.$; Figure 5.9a), but neither a main effect of sex nor a sex by maternal treatment interaction $(p>0.05)$. Planned comparisons showed that saline-injected, BPA-exposed animals had significantly reduced POMC fibre density in the PVN relative to Control animals $(\phi<0.05)$. Leptin-treated animals showed a significant main effect of sex $\left(F_{(1,24)}=15.971, p<0.001\right.$; Figure 5.9a), but no effect of maternal treatment, nor an interaction between the two $(p>0.05)$. The main effect of sex in these animals can be attributed to a markedly stronger response to leptin injection on the part of females. While leptin-injected females exposed to each of the three maternal treatments showed increased POMC fibre density relative to their saline controls, the effect was greatest in BPA-exposed animals where leptin treatment restored fibre density to levels equivalent to Controls. We did not detect any treatment-, sex-, or injection-related differences in AgRP fibre density ( $p>0.05$; Figure 5.9b).

\subsection{DISCUSSION}

In Chapters 3 and 4 we noted that BPA-exposed males and females showed, in adulthood, a number of markers of leptin insensitivity both before and after diet-induced obesity. Moreover, we noted that BPA exposure was associated with reductions in POMC immunoreactive fibre density in the PVN, a neuromorphological correlate common to many developmental models of obesity. In this chapter, we endeavoured to study the ontogeny of these effects in more detail with the aim of identifying a developmental period or process to which most of BPA's effects could be attributed.

We began by studying the effect of BPA on prenatal neurogenesis, measured by the incorporation of $\mathrm{BrDU}$ given at E12. We quantified $\mathrm{BrDU}$ incorporation in hypothalamic feeding nuclei in animals sacrificed at P16 and found no evidence of altered neurogenesis in any of the treatment conditions, or in either of the sexes (see Figure 
5.3). Though we did not exhaustively break down BrDU labelling by cell phenotype, we did explore co-labelling of $\mathrm{BrDU}$ and POMC in pups sacrificed at P21, knowing POMC transcriptional activity and axonal outgrowth to be important targets of BPA and DES (see Chapters 3 and 4, and Figure 5.5b). Again we found no differences either in the absolute number of POMC neurons, nor in the number of POMC neurons labelled with BrDU (see Figure 5.4). Of note, there were no sex differences in the number of POMC neurons at this timepoint, which is in contrast to our earlier finding that adult female mice had more POMC neurons than males (see Figure 3.10). On this basis, it is possible to speculate that the adult sex difference comes about not through neurogenesis but instead through a mechanism such as apoptosis later in life.

Our study of POMC-expressing fibres in the PVN began with animals from Cohort 1 sacrificed at P21, the same animals, in fact, as were used to quantify POMC neuron numbers in the ARC. We found the density of these fibres to be greater in females generally, but reduced by BPA exposure in both sexes (Figure 5.5b). The sex difference we observed in POMC staining intensity has been reported previously, as has its susceptibility to disruption by sex steroids [238]. There are some differences between these results in P21 animals and those we found in adult animals in Chapter 3 , most notably the fact that the effect of BPA was only evident in adult males (see Figure 3.9a). Since the measurements were carried out at different timepoints (P21 versus P105), it is likely that the difference emerges at some point in the period that intervenes between weaning and adulthood. While the majority of ARC-derived fibres have assumed an adult-like arrangement by P21, there does seem to be some continued growth into adulthood [286]. Some of these fibres may be late arrivals from the ARC, but it could also be that the nucleus of the solitary tract (NTS), which projects to the PVN [319] and contains POMC-expressing neurons [320], makes up the difference in PVN innervation once its efferents develop. Our use of immunofluorescent labelling rather than orthograde tracing does not distinguish among different points of origin, making the provenance of these fibres somewhat uncertain. Nevertheless, reduced anorectic innervation of the PVN, a key site in the homeostatic control of feeding, is likely to play a role in the behavioural manifestations of leptin insensitivity we observed in Chapter 4.

We discovered that animals exposed to BPA and DES showed a neonatal leptin surge that was respectively delayed and absent relative to Controls (see Figure 5.6). To our knowledge, this is the first report of a disrupted leptin surge in the absence of any nutritional manipulation to either the dam or pup. Differences in leptin secretion cannot be attributed to differences in nutrition, growth, or litter size, for our BPAand DES-exposed pups were equivalent to Controls in all of those regards (see Figure 5.2). Our data (see Figure 5.6b), as well as other published reports ([197; 321]) show that the surge in plasma leptin in Control animals is mirrored by an increase in leptin transcription in the pup's white adipose tissue. This is not so either in BPA- 
or DES-treated animals. BPA-treated animals also did not show an increase in leptin transcription that matched their delayed leptin surge, but this may be due to adipose tissue depot-specific leptin expression. Presently it is not known how the leptin surge is generated in the first place, nor, therefore, is it known how it comes to be disrupted by nutritional or chemical influences. Since both BPA and DES have estrogenic effects, the fact that they produce similar phenotypes here may indicate that our observed effect, and perhaps even the surge itself, depends on estrogen receptor signalling. On the other hand, it may simply be that the two compounds affect adipogenesis in similar ways. It is true that both BPA and DES induce adipogenesis in the 3T3-L1 cell line, increasing the expression of adipocyte-specific markers and the accumulation of triglycerides[119; 106; 159], but the effect seems to be estrogenic in the case of DES [159] and glucocorticoid receptor-mediated in BPA [119]. If the onset and timing of the leptin surge is several steps removed from the receptors upon which the two compounds act, then perhaps it depends on the developing adipose tissue passing some growth-related milestone that is normally met early in life but retarded or advanced by our compounds (and, for that matter, adverse nutritional environments).

Adult BPA- and DES-treated animals have impaired adult leptin sensitivity in common (see Chapter 4), and given the primacy of appropriate early-life leptin exposure in determining the adult response to leptin [206; 207; 322; 197], the disrupted leptin surge seen in these animals may be explanatory in this regard. Perinatal leptin is also thought to play a role in guiding the development of efferent projections from neurons in the ARC to other nodes in the feeding circuitry [301], and since these pathways were disrupted in our BPA-exposed animals, we decided to conduct a study in which pups exposed to our test compounds received supplemental leptin by way of daily subcutaneous injection. Here we found that neonatal leptin supplementation rescued the reduced POMC projections in BPA-exposed animals in females but not males (see Figure 5.9). AgRP projections were not altered by BPA or DES, nor were they affected by leptin supplementation. Taken together with the data showing a disrupted leptin surge in BPA- and DES-exposed animals, our results suggest that reduced neonatal leptin secretion cannot be entirely responsible for the reductions in POMC projections seen in BPA-exposed animals. This is because neonatal leptin secretion was also low in DES-exposed animals, yet the POMC projections developed appropriately in these animals. This we see as evidence that the effect must be at the level of the developing neurons themselves. Given the diversity of BPA's pharmacological activity, it is beyond the scope of this paper to directly implicate a single receptor system as the key mediator of this effect. However, certain inferences can be made by comparing the results of DES-exposed mice. Since the estrogenic nature of DES is rather more pure and potent than that of BPA, it is unlikely that our observations are mediated by the estrogen receptor. They may, on the other hand, be mediated by the androgen receptor, upon which BPA has been reported to have both agonistic [90] and antagonistic [85] 
effects. In this way, BPA may mimic the effects of neonatal dihydrotestosterone exposure, which also programs energy balance via the POMC neurons [238]. That neonatal leptin supplementation rescued the POMC projections in a sex-specific manner may be due to it working on a background of pre-existing sex differences. It is important to consider that male and female rodents differ in their susceptibility to the various models of developmental programming [323; 324; 325]. Perhaps as a result of these sex differences, there have been numerous other reports of sexually dimorphic responses to supplemental leptin during the neonatal period with females generally showing a greater response in adult POMC expression [313], as well as a reduced propensity for adult diet-induced obesity in models of perinatal undernutrition $[316 ; 326]$. The origin of these sex differences is not known at present, but in light of the fact that peripheral leptin concentrations do not show sex differences in the neonatal period, it may be a cell-autonomous feature of the POMC neurons.

In summary, we have provided evidence that many aspects of the adult BPAexposed phenotype develop during the early postnatal period. Embryonic neurogenesis of POMC neurons is apparently not affected by our model, but BPA does inhibit the postnatal wiring of these neurons in addition to the surge in circulating leptin that accompanies this event. We have further shown that, due to incongruities with the effects of DES exposure, the reduced melanocortin projections of the BPA-induced phenotype are unlikely to be estrogenic in origin, while the disrupted leptin surge likely is. 
(a)

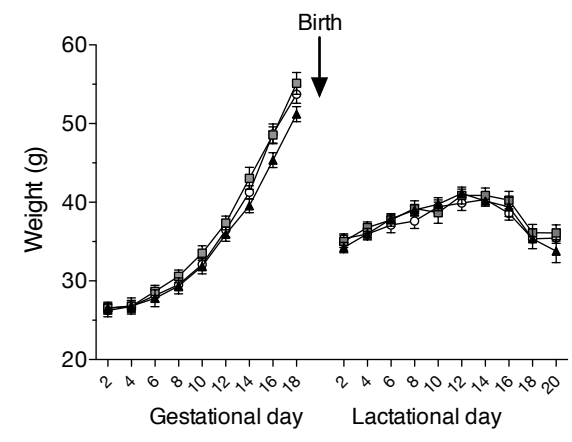

(c)

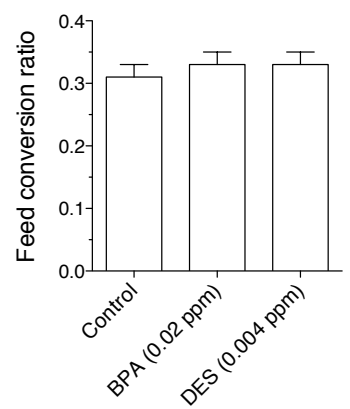

(f) (b)

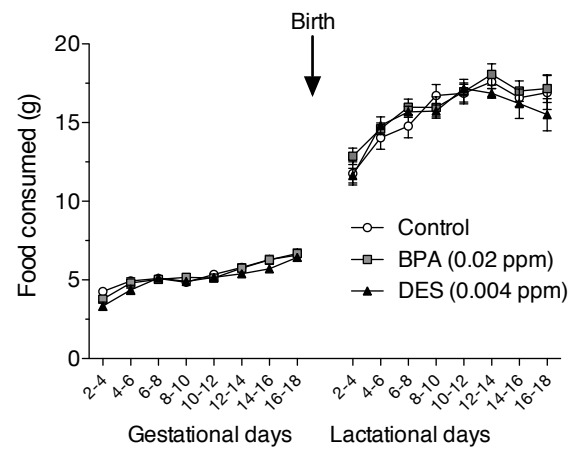

(e) (d)
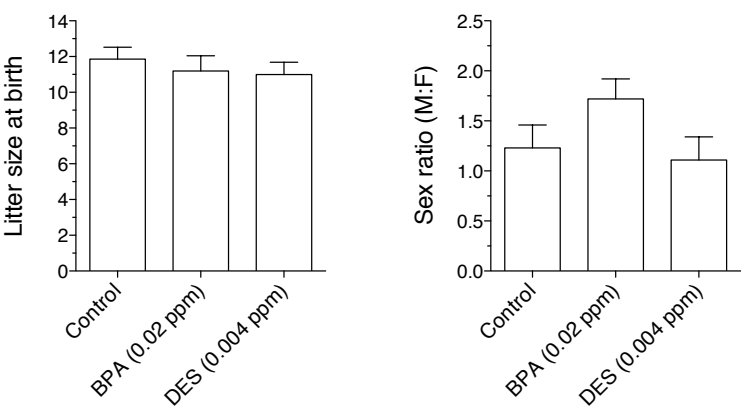

(g)
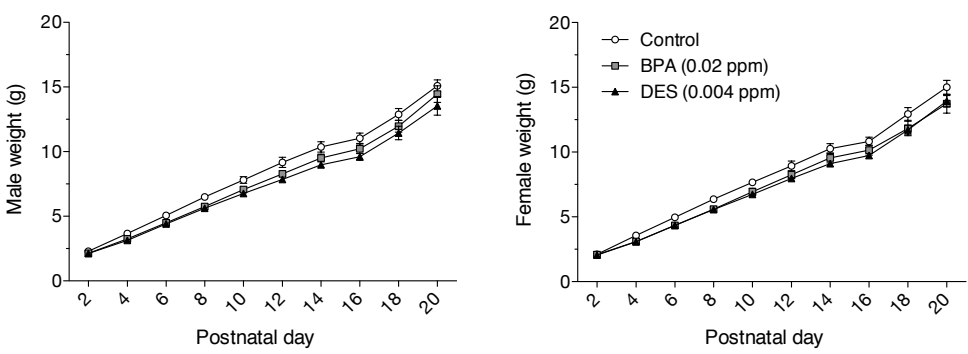

Figure 5.2: Maternal and litter data.

Maternal body weight (a) and food intake (b) during the gestational and lactational periods. Maternal feed conversion ratio (c) during the gestational period. Average litter size (d) and sex ratio at birth (e). Male (f) and female (g) pup body weights during the postnatal period. All values expressed as mean \pm SEM. 


\begin{tabular}{llll}
\hline & Control & BPA $(0.02 \mathrm{ppm})$ & DES $(0.004 \mathrm{ppm})$ \\
\hline G1-7 & $0 \pm 0$ & $3.25 \pm 0.14$ & $0.62 \pm 0.03$ \\
G8-14 & $0 \pm 0$ & $3 \pm 0.11$ & $0.78 \pm 0.17$ \\
G15-21 & $0 \pm 0$ & $2.66 \pm 0.08$ & $0.77 \pm 0.24$ \\
Gestational days average & 0 & 2.97 & 0.72 \\
\hline L0-6 & $0 \pm 0$ & $7.51 \pm 0.21$ & $1.47 \pm 0.06$ \\
L7-13 & $0 \pm 0$ & $8.38 \pm 0.34$ & $1.64 \pm 0.04$ \\
L14-21* & $0 \pm 0$ & $8.4 \pm 0.47$ & $1.65 \pm 0.08$ \\
Lactational days 0-13 average & 0 & 7.94 & 1.56 \\
\hline
\end{tabular}

Table 5.1: Estimated maternal doses of BPA and DES Weekly doses of each compound $(\mu \mathrm{g} / \mathrm{kg} /$ day) estimated using maternal food intake and body weight, and that compound's concentration in the diet.

* At P14 pups begin reaching for solid food, so these values represent whole-cage food disappearance. 
(a)

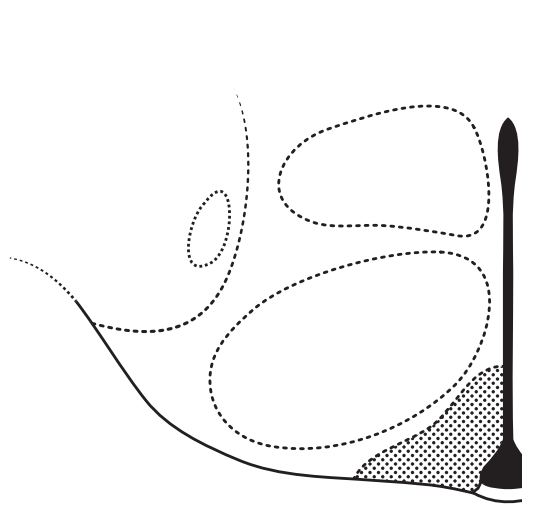

(c) Control female

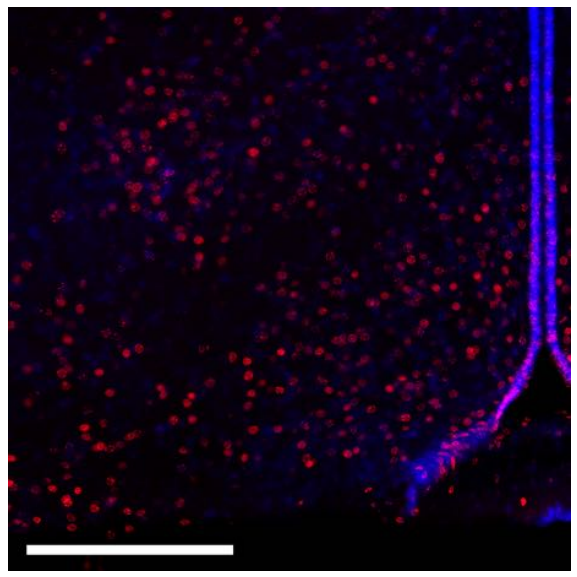

(b)

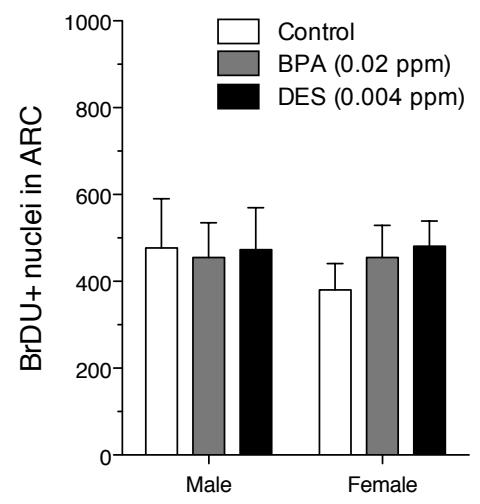

(d) BPA (0.02 ppm) female

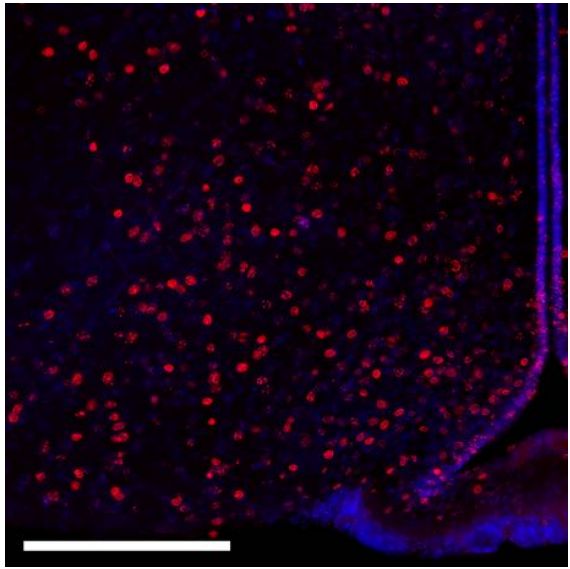

Figure 5.3: BrDU immunofluorescence at P16.

BrDU labeled cells in ARC (b); female Control (c), BPA (d). All photomicrographs captured at 10x magnification with DAPI nuclear counterstaining, scale bars $=250 \mu \mathrm{m}$. All values expressed as mean \pm SEM. Continued... 
(e)

(f)
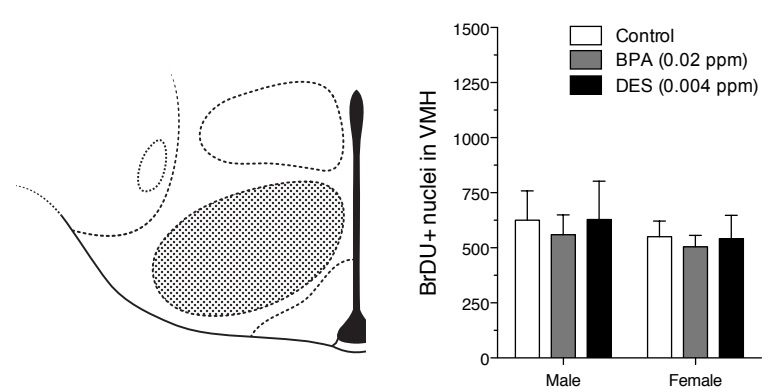

(j)
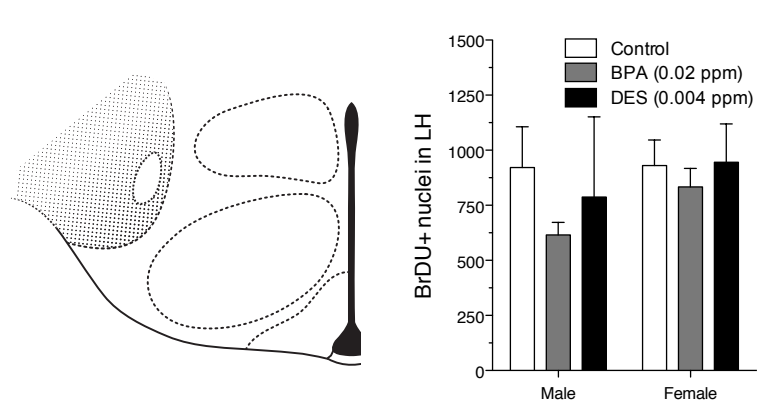

(g) Control female

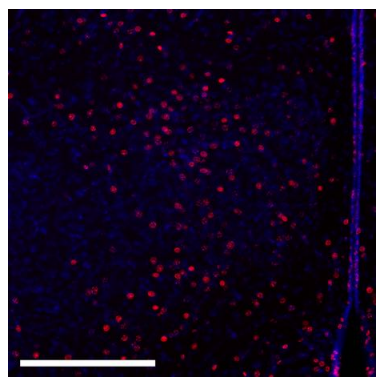

(k) Control female

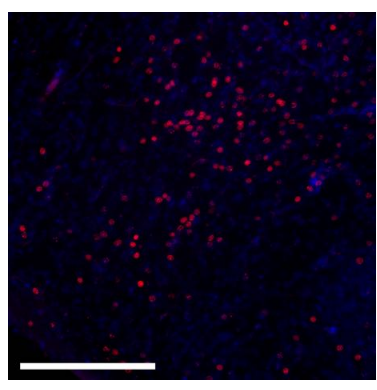

(l) BPA (0.02 ppm) female

(h) BPA (0.02 ppm) female
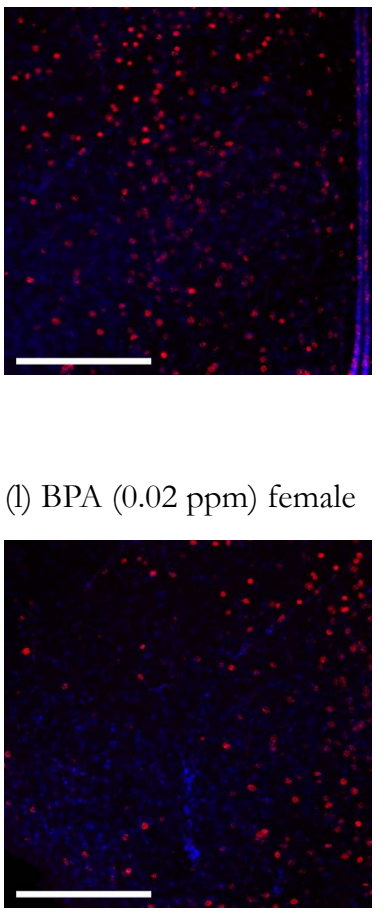

Figure 5.3: Continued. BrDU immunofluorescence at P16.

BrDU labeled cells in VMH (f); female Control (g), BPA (h). LH (j); female Control (k), BPA (l). All photomicrographs captured at 10x magnification with DAPI nuclear counterstaining, scale bars $=250$ $\mu \mathrm{m}$. All values expressed as mean \pm SEM. Continued... 
$(\mathrm{m})$

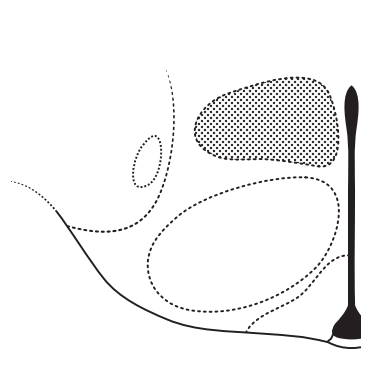

(n)

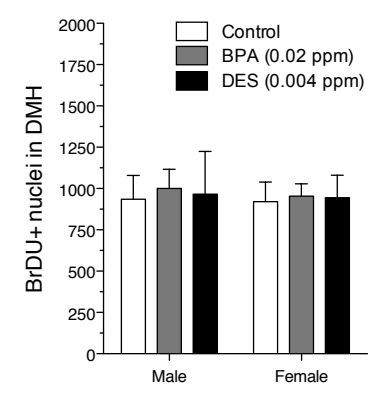

$(\mathrm{r})$

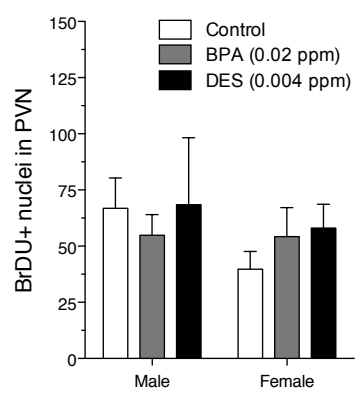

(q)

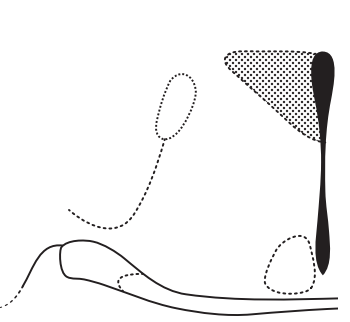

(p) BPA (0.02 ppm) female (o) Control female

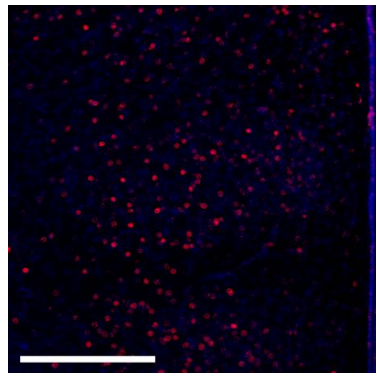

(n)

(s) Control female

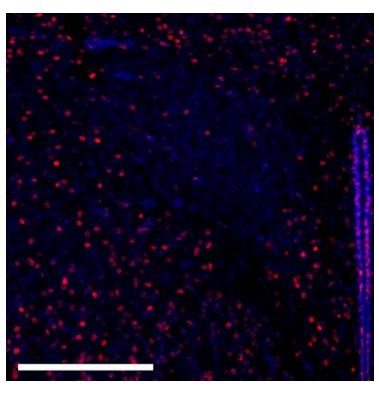

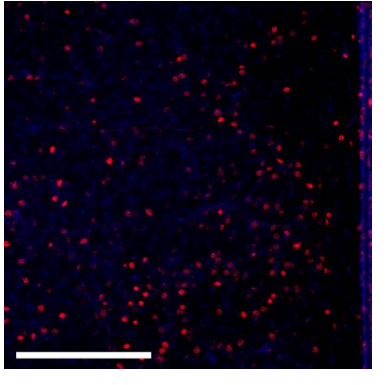

(t) BPA (0.02 ppm) female

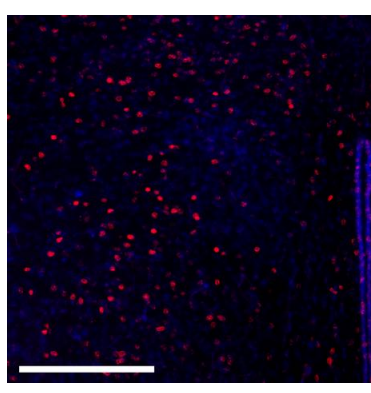

Figure 5.3: Continued. BrDU immunofluorescence at P16.

BrDU labeled cells in DMH (n), female Control (o), female BPA (p). PVN (r), female Control (s), BPA

(t). All photomicrographs captured at 10x magnification with DAPI nuclear counterstaining, scale bars $=250 \mu \mathrm{m}$. All values expressed as mean \pm SEM. 
(a)

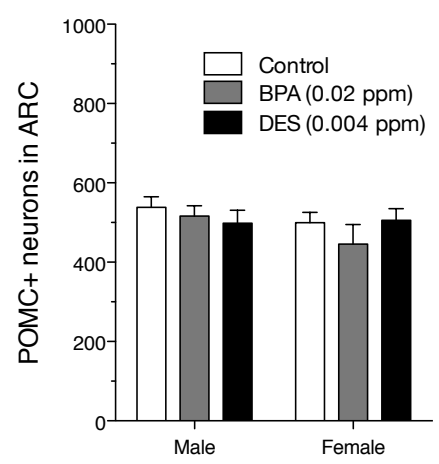

(d) POMC - Control female

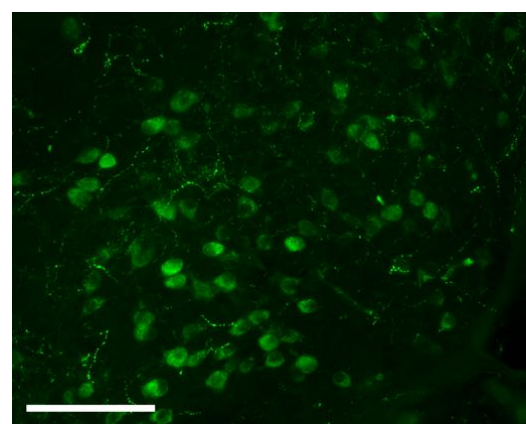

(e) BrDU - Control female

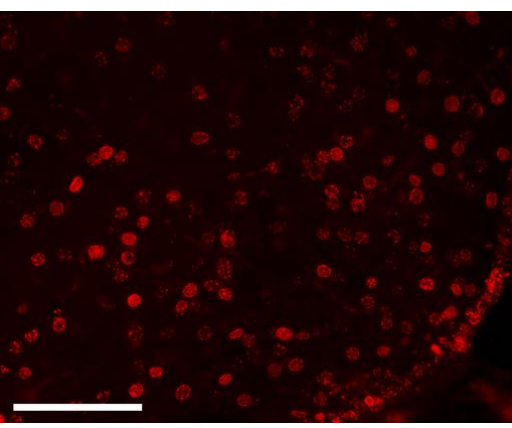

(g) POMC - BPA (0.02 ppm) female

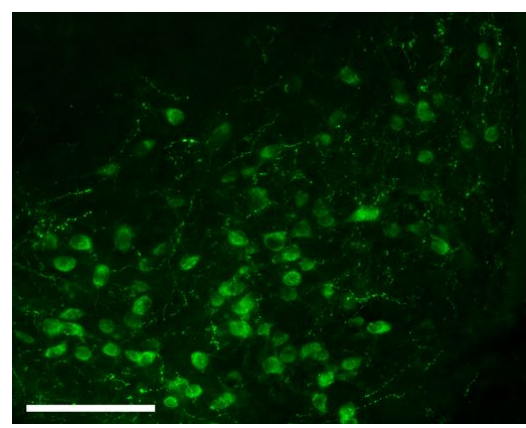

(h) BrDU - BPA (0.02 ppm) female

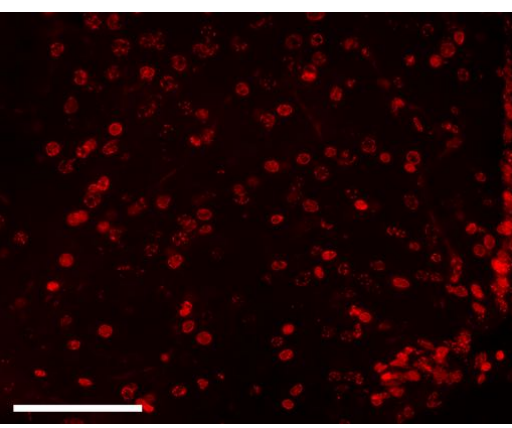

(c)

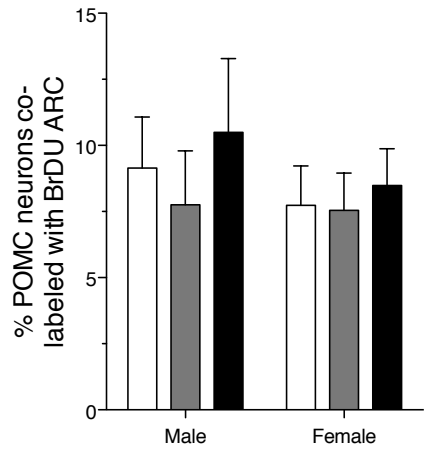

(f) Merged - Control female

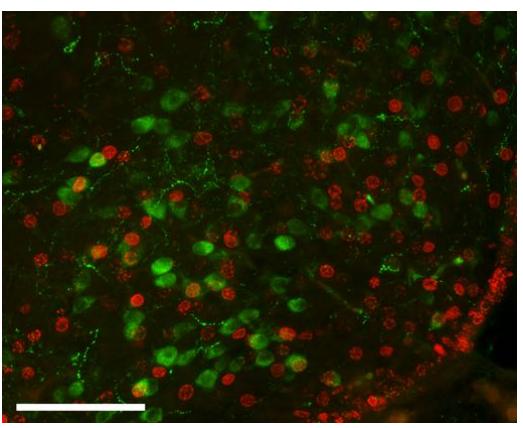

(i) Merged - BPA (0.02 ppm) female

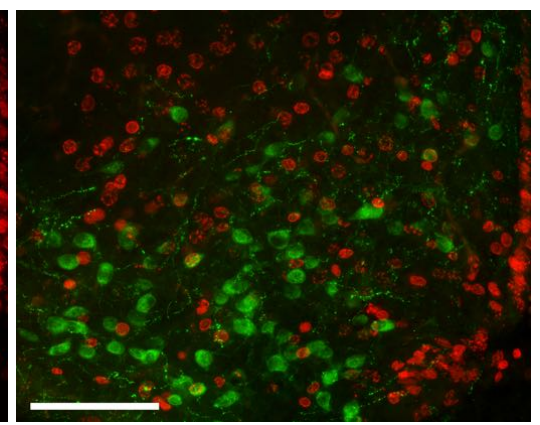

Figure 5.4: Colocalization of BrDU and POMC in the ARC at P21.

Quantification of POMC (a) and BrDU (b) immunoreactive cells in the ARC. per cent of total POMC immunoreactive neurons colocalized with BrDU (c). Female control POMC (d), BrDU (e), merged (f). Female BPA POMC (g), BrDU (h), merged (i). All photomicrographs captured at 20x magnification, scale bars $=75 \mu \mathrm{m}$. All values expressed as mean \pm SEM. 
(a)

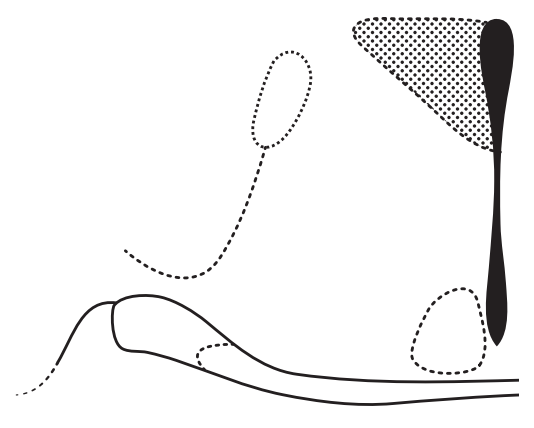

(b)

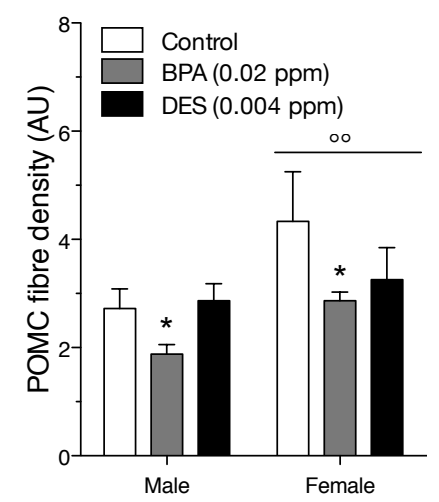

(c) Control male

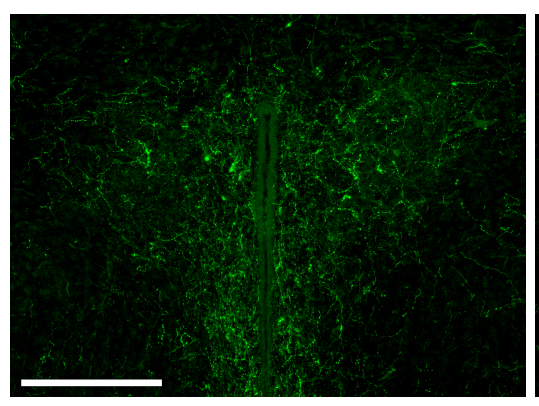

(d) BPA (0.02 ppm) male

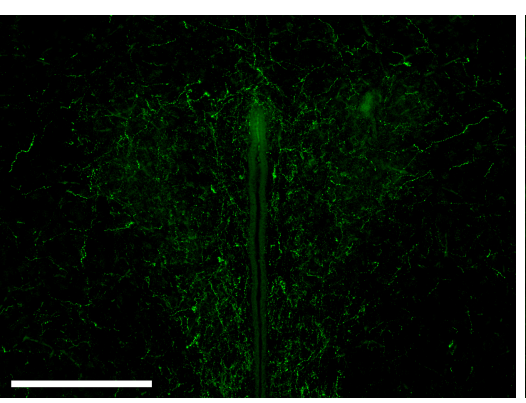

(g) BPA (0.02 ppm) female

(f) Control female

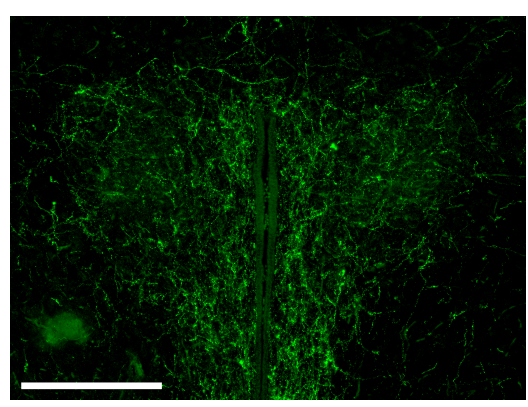

(e) DES (0.004 ppm) male

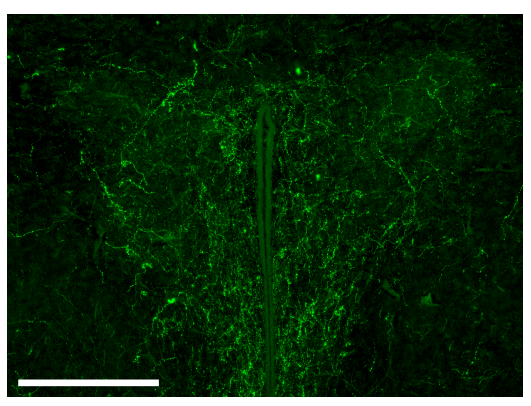

(h) DES (0.004 ppm) female

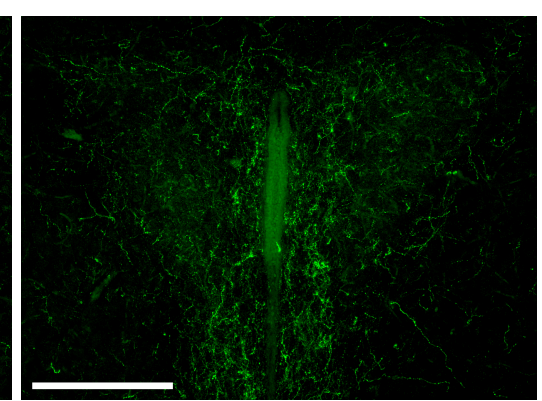

Figure 5.5: POMC projections to the PVN at P21.

POMC immunolabeled fibres in the PVN (arbitrary units) (b). Male control (c), male BPA (d), male DES (e); female control (f), female BPA (g), female DES (h). All photomicrographs captured at 10x magnification, scale bars $=250 \mu \mathrm{m}$. $* p<0.05$ vs. control animals of the same sex. All values expressed as mean \pm SEM. 
(a)

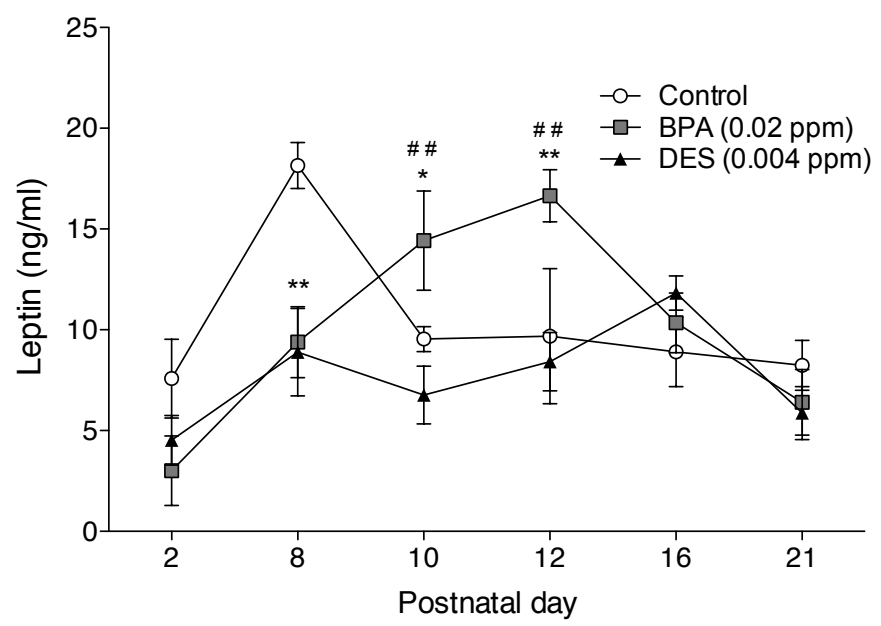

(b)

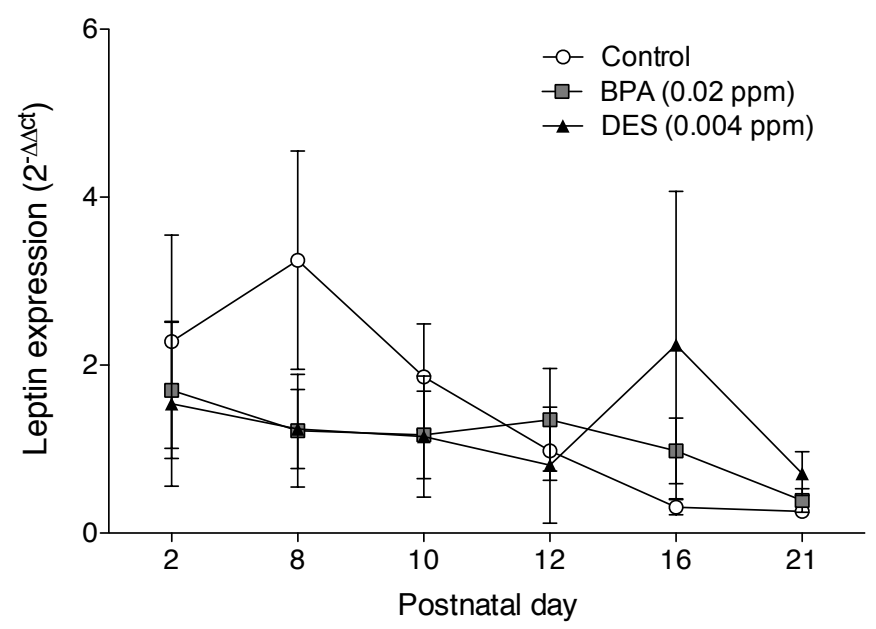

Figure 5.6: Postnatal leptin surge.

Circulating leptin in trunk blood at postnatal days 2, 8, 10, 12, 16, and 21 (a). Leptin mRNA in white adipose tissue at postnatal days $2,8,10,12,16$ and 21 (b). $* p<0.05$ vs. Control, $* * p<0.01$ vs. Control; \# $\not<0.05$ vs. DES, \#\# $<0.01$ vs. DES. All values expressed as mean \pm SEM. 
(a)

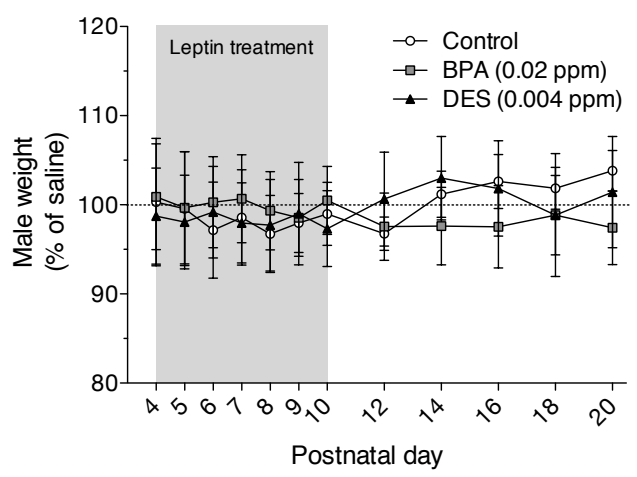

(b)

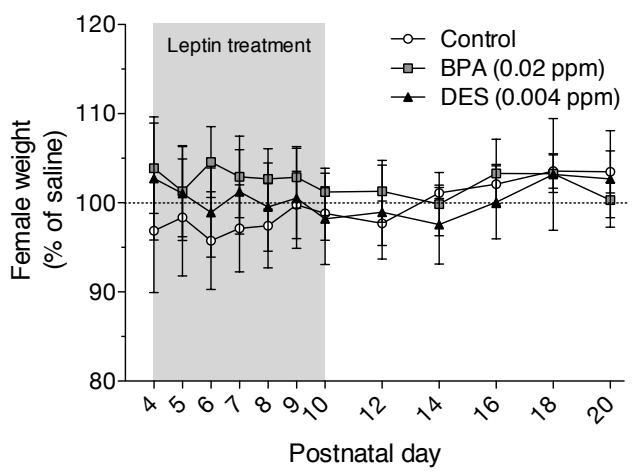

Figure 5.7: Relative body weight of leptin-treated animals.

Body weight expressed as a percent of the average weight of sex-matched saline-treated animals from the same group. Body weight was measured daily over the period of leptin injection (P4-10), then on alternating days from P12-20. Males (a); females (b). All values expressed as mean \pm SEM. 
(a) Male

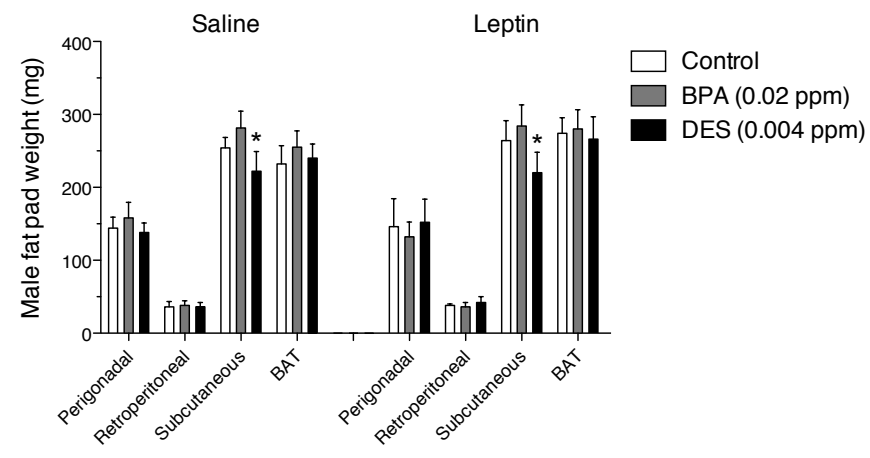

(b) Female

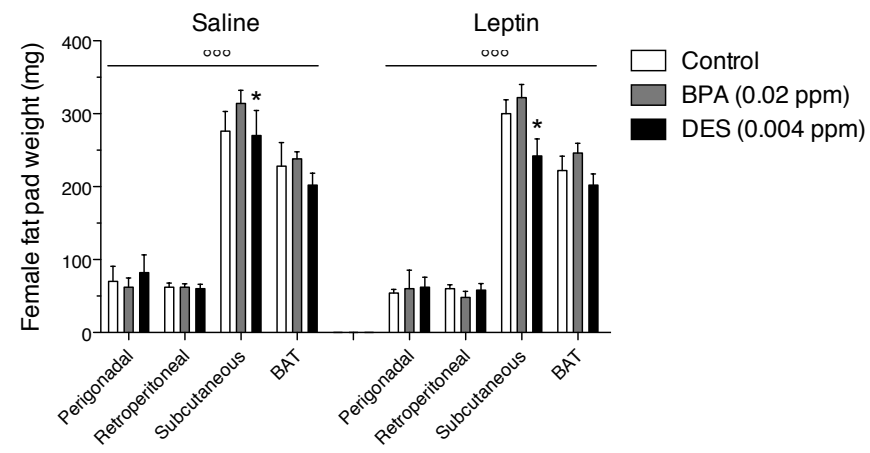

Figure 5.8: Fat pad mass at P21.

Mass of the perigonadal, retroperitoneal, subcutaneous, and brown adipose tissue (BAT) fat pads in salineand leptin-treated pups sacrificed at P21. Males (a); females (b). $* p<0.05$ vs. same-sex Control; $\circ \circ \circ p<0.001$ vs. male animals from the same treatment group. All values expressed as mean $\pm \mathrm{SEM}$. 
(a)

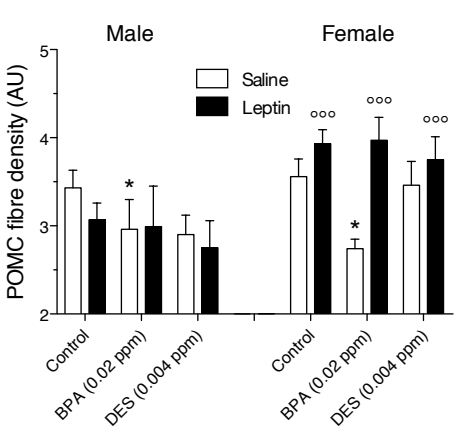

(b)

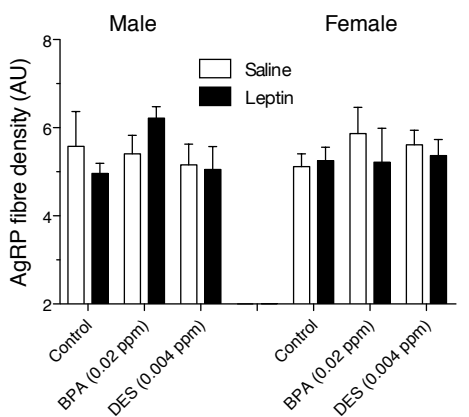

(c)

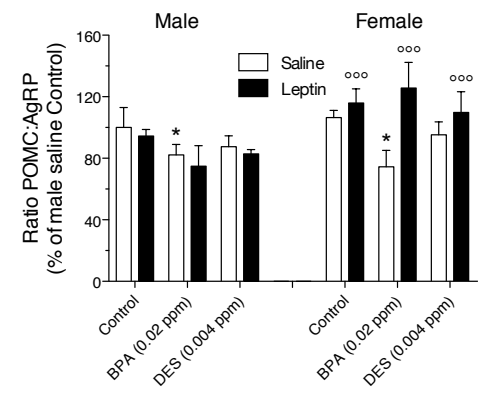

(d) POMC - Con female S (e) POMC - Con female L

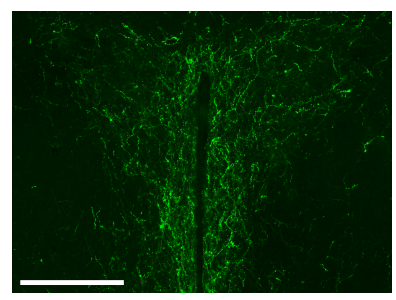

(h) AgRP - Con female S

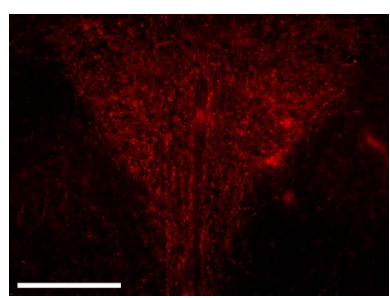

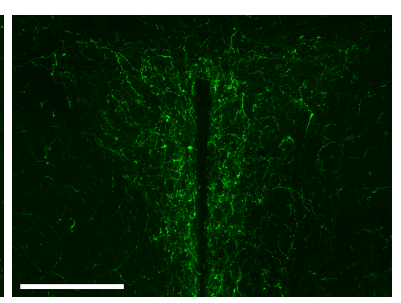

(i) AgRP - Con female L

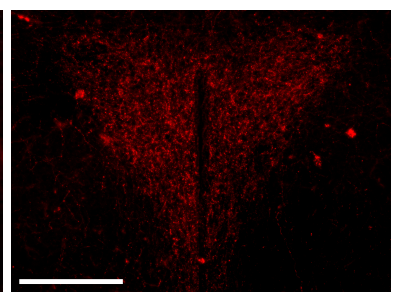

(f) POMC - BPA female S

(g) POMC - BPA female L
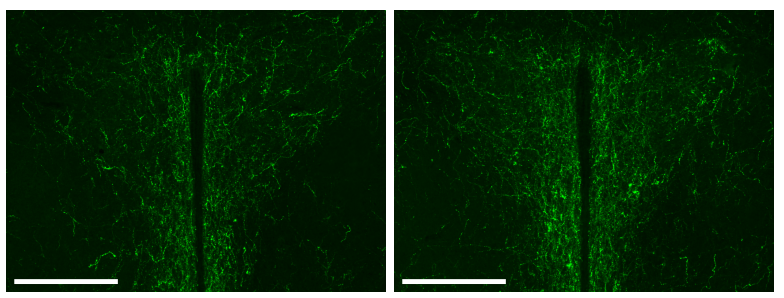

(j) AgRP - BPA female $S$

(k) AgRP - BPA female L
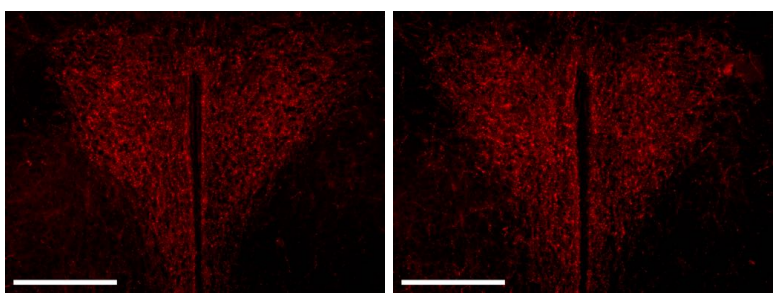

Figure 5.9: POMC and AgRP projections to the PVN in leptin-treated animals.

POMC fibre density in saline- and leptin-treated animals (arbitrary units) (a); AgRP fibre density (b); measure of the ratio between POMC and AgRP staining in each section (c). Representative images showing POMC staining in female saline (d) and leptin (e) Control animals, female saline (f) and leptin (g) BPA-treated animals. AgRP staining in female saline (h) and leptin (i) Control animals, female saline (j) and leptin (k) BPA-treated animals. $* p<0.05$ vs. saline-treated Control; $\circ \circ \circ p<0.001$ vs. leptintreated male animals from the same group. All photomicrographs captured at 10x magnification, scale bars $=250 \mu \mathrm{m}$. All values expressed as mean \pm SEM. 


\section{Chapter 6}

\section{General Discussion}

The present thesis set out to first develop a model of environmentally relevant perinatal exposure, and then to explore its effects on adult metabolism, food intake, and the neural circuits that mediate the two. As a whole, this body of work suggests that the end result of our model of exposure consists of sex-specific metabolic impairments in adulthood, accompanied by functional changes in leptin sensitivity, and structural changes in the leptin-sensitive anorexigenic melanocortin circuitry. Deficits in the postnatal leptin surge likely account for some, but not all aspects of this phenotype, but the genesis of these deficits, as well as the other phenotypic effects that they do not explain remains uncertain. It is beyond the scope of this project to experimentally dissect the signalling pathways through which BPA exerts its various organizational effects, though such an extension is certainly possible given the basis we have established here. Scope and uncertainty notwithstanding, it is possible to infer a great deal about the likely mechanisms of BPA's actions from our results, and this is the aim of the following discussion.

\subsection{IS BPA ACTING AS A XENOESTROGEN?}

BPA is conventionally regarded as a xenoestrogen, and we have fashioned our studies around the assumption that its estrogenic properties are the most relevant for its effects on body weight. To this end we have included DES as an estrogenic positive control in each study. We have also carried out all of our analyses on both male and female animals, while paying particular attention to the possible reversal or enhancement of any sex-specific characteristics, as might be expected of an estrogen mimic. Given what is now known about BPA pharmacology (see Section 1.3), this was only one choice out of many other valid possibilities.

\subsubsection{Inferences From Sex Differences}

Within the brain there are a number of regions that exhibit reliable sexual dimorphisms that develop under the control of reasonably well understood hormonal influences. 
The influence of BPA on these circuits remains uncertain, as relevant studies abound with differences in methodology and outcome (see Section 1.4). Nevertheless, if BPA is acting in the capacity of a sex steroid mimic, then every observable sex difference is a potential testbed for that hypothesis.

Given that masculinization is mediated in large part by estrogen receptor signalling, a key expectation of the hypothesis that BPA affects the sexual differentiation of the feeding circuitry is that Control females differ substantially from Control males on certain measures, and that BPA- or DES-exposed females show responses that are equivalent to Control males on those same measures. That is, xenoestrogen-exposed females should show a reversal of certain sexually dimorphic characteristics. Only on rare occasions did we find this to be the case. More often, what sex differences we found in the brain's feeding circuitry remained in place despite BPA treatment. For example, compared to males females had significantly more vGlut1 immunopositive terminals onto POMC neurons in the ARC, and this was not affected by our treatments (Figure 3.9g). Adult females had more POMC neurons than males, and this was also not affected by any of our treatments (Figure 3.10b). Females showed a markedly higher rate of rebound feeding than males, and this example of sexually dimorphic feeding behaviour was not reversed by any of our treatments (Figure 4.5). Diet-induced obese males showed significant reductions in liver glycogen content, but these remained nearly at control levels in all females, and this too was not affected by our treatments (Figure $3.7 b)$.

Two notable exceptions stand out in that they do seem to be bonafide sex differences that are altered or absent in BPA-treated animals. Firstly, males had a higher percentage of POMC neurons co-expressing ER $\alpha$ than females, and this was reversed by BPA treatment (Figure 3.10c). Secondly, females had denser POMC immunoreactivity in the PVN, but BPA-exposed females were brought to levels equivalent to Control males (Figure 5.5) These fibres also showed a markedly stronger response to neonatal leptin in females, with BPA-exposed females showing the greatest relative response of all (Figure 5.9a). While these two examples could be interpreted within the framework of sexual differentiation, and indeed we argue that sex hormone receptors are likely involved in the development of the POMC fibres, this is not the only possible explanation. It could be that leptin is the effector of these and other changes we observed, since leptin levels were disrupted at a developmentally crucial time ${ }^{1}$. But leptin is not seen as a sex hormone in most circles, and its secretion during neonatal life does not differ between the sexes. Leptin does play an indirect role in many as-

\footnotetext{
${ }^{1}$ It is also possible that the developmental alterations in leptin secretion and leptin sensitivity could account for many of the BPA-induced reproductive and hippocampal abnormalities reported by others. As an example, perinatal BPA treatment decreases NMDA receptor subunit NR1 and NR2A expression in the adult hippocampus [327], and those same subunits are upregulated in adults neonatally supplemented with leptin [328].
} 
pects of reproductive and sexual behaviour, for example playing a permissive role in the onset of puberty $[329 ; 330]^{2}$, but this is several steps removed from the way sexual differentiation is usually conceived.

Sex differences in feeding and energy homeostasis have been studied for decades, but with the advent of molecular biology, transgenic mouse models, and an understanding of the central regulation of energy balance that is becoming ever more sophisticated, our conception of sex differences in these systems is far from caught up, and far from being canonized. Thus we are not in a position to point to an enlarged nucleus here, or an increase in dendritic arborization there, and tie it to a vast foundation of prior research. This only serves as a testament to the need for more research into the topic, starting perhaps with the inclusion of both males and females in transgenic phenotyping studies ${ }^{3}$.

\subsubsection{Inferences From the Effects of DES}

Instances of concordance between BPA- and DES-treated animals suggest that similar mechanisms may be at work behind both phenotypes. Of course, additional work is needed to confirm this supposition, but the ability to make such inferences is a key advantage of employing a well understood positive control. As it stands, we have found a few examples of concordance between our BPA-treated animals and those treated with DES, but far more often we found these animals to be discordant. Instances of concordance between the effects of BPA and DES (excluding instances when neither of them have an effect) are summarized in Table 6.1.

One of the most notable instances of concordance involved our observation of adult leptin insensitivity. As we mentioned in Chapter 5, the type of leptin insensitivity we observed is strikingly similar to that reported by Nohara et al., after neonatal exposure to testosterone. The testosterone-exposed phenotype itself is the combination of estrogenic and androgenic stimulation, as testosterone is a prohormone that can be irreversibly converted to either a pure estrogen (17 $\beta$-estradiol) or androgen (dihydrotestosterone). When the phenotype was parcelled out into its constituents, the authors found that leptin resistance came about by estrogenic stimulation [238]. Analogously, exposure to DES produced a similar phenotype of leptin resistance in our animals, and since this was matched in BPA-exposed animals, it is reasonable to label it an estrogenic effect.

\footnotetext{
${ }^{2}$ In fact, the permissive effects of leptin on puberty onset are disrupted by neonatal DES exposure in a way that both mimics and synergizes with food restriction [331]. The substantial overlap between reproductive and energy balance circuitry makes such findings difficult to classify as belonging to one or the other.

${ }^{3}$ When done, this approach quite often yields valuable information, such as when Shi et al., found that specific deletion of $\mathrm{ObRb}$ in POMC neurons produced impaired glucose tolerance in males but not females [332; 235].
} 


\begin{tabular}{|c|c|c|c|c|c|}
\hline & Sex & BPA & DES & Concordance & Figure \\
\hline Susceptability to diet-induced obesity & $\begin{array}{l}\mathrm{M} \\
\mathrm{F}\end{array}$ & . & . & & $\begin{array}{l}3.2 \mathrm{a} \\
3.2 \mathrm{~b}\end{array}$ \\
\hline High-fat diet-induced hyperphagia & $\begin{array}{l}\mathrm{M} \\
\mathrm{F}\end{array}$ & 唐 & . & & $\begin{array}{l}3.2 \mathrm{c} \\
3.2 \mathrm{~d}\end{array}$ \\
\hline Fat mass (post-HFD) & $\begin{array}{c}\mathrm{M} \\
\mathrm{F}\end{array}$ & $\begin{array}{l}\Uparrow \\
\Uparrow\end{array}$ & . & & $\begin{array}{l}3.3 \mathrm{c} \\
3.3 \mathrm{~d}\end{array}$ \\
\hline Energy expenditure & $\begin{array}{l}\mathrm{M} \\
\mathrm{F}\end{array}$ & $\Uparrow$ & . & & $\begin{array}{l}3.4 b ; 3.5 a \\
3.4 d ; 3.5 b\end{array}$ \\
\hline Glucose tolerance & $\begin{array}{l}\mathrm{M} \\
\mathrm{F}\end{array}$ & $\Downarrow$ & . & & $\begin{array}{l}3.6 \mathrm{a} \\
3.6 \mathrm{~b}\end{array}$ \\
\hline Insulin (post-HFD exposure) & $\begin{array}{l}\mathrm{M} \\
\mathrm{F}\end{array}$ & $\Uparrow$ & 介. & $\checkmark$ & $\begin{array}{l}3.4 \\
3.4\end{array}$ \\
\hline Leptin (post-HFD exposure) & $\begin{array}{l}\mathrm{M} \\
\mathrm{F}\end{array}$ & . & . & & $\begin{array}{l}3.8 \mathrm{a} \\
3.8 \mathrm{a}\end{array}$ \\
\hline POMC fiber density in PVN (adult) & $\begin{array}{c}\mathrm{M} \\
\mathrm{F}\end{array}$ & $\Downarrow$ & $\dot{v}$ & & $\begin{array}{l}3.9 \mathrm{a} \\
3.9 \mathrm{a}\end{array}$ \\
\hline POMC ER $\alpha$ co-localization & $\begin{array}{l}\mathrm{M} \\
\mathrm{F}\end{array}$ & . & . & & $\begin{array}{l}3.10 \mathrm{c} \\
3.10 \mathrm{c}\end{array}$ \\
\hline ER $\alpha$ expression in ARC (post-HFD) & $\begin{array}{l}\mathrm{M} \\
\mathrm{F}\end{array}$ & 放 & . & & $\begin{array}{l}3.11 \mathrm{a} \\
3.11 \mathrm{a}\end{array}$ \\
\hline POMC expression in ARC (post-HFD) & $\begin{array}{c}\mathrm{M} \\
\mathrm{F}\end{array}$ & $\dot{v}$ & $\Downarrow$ & $\checkmark$ & $\begin{array}{l}3.11 \mathrm{~b} \\
3.11 \mathrm{~b}\end{array}$ \\
\hline AgRP expression in ARC (post-HFD) & $\begin{array}{l}\mathrm{M} \\
\mathrm{F}\end{array}$ & $\Uparrow$ & . & & $\begin{array}{l}3.11 \mathrm{c} \\
3.11 \mathrm{c}\end{array}$ \\
\hline SOCS3 expression in ARC (post-HFD) & $\begin{array}{c}\mathrm{M} \\
\mathrm{F}\end{array}$ & 丹. & i & & $\begin{array}{l}3.11 \mathrm{f} \\
3.11 \mathrm{f}\end{array}$ \\
\hline STAT3 expression in ARC (post-HFD) & $\begin{array}{l}\mathrm{M} \\
\mathrm{F}\end{array}$ & . & $\Uparrow$ & & $\begin{array}{l}3.11 \mathrm{~g} \\
3.11 \mathrm{~g}\end{array}$ \\
\hline Leptin sensitivity (overnight weight loss) & $\begin{array}{l}\mathrm{M} \\
\mathrm{F}\end{array}$ & $\Downarrow$ & $\begin{array}{l}\Downarrow \\
\Downarrow\end{array}$ & $\begin{array}{l}\checkmark \\
\checkmark\end{array}$ & $\begin{array}{l}4.7 \mathrm{a} \\
4.7 \mathrm{a}\end{array}$ \\
\hline Leptin-induced POMC expression & $\begin{array}{l}\mathrm{M} \\
\mathrm{F}\end{array}$ & $\begin{array}{l}\Downarrow \\
\Downarrow\end{array}$ & $\begin{array}{l}\Downarrow \\
\Downarrow\end{array}$ & $\begin{array}{l}\checkmark \\
\checkmark\end{array}$ & $\begin{array}{l}4.8 \mathrm{a} \\
4.8 \mathrm{a}\end{array}$ \\
\hline POMC fiber density in PVN (P21) & $\begin{array}{l}\mathrm{M} \\
\mathrm{F}\end{array}$ & $\Downarrow$ & . & & $\begin{array}{l}5.5 \mathrm{~b} ; 5.9 \mathrm{a} \\
5.5 \mathrm{~b} ; 5.9 \mathrm{a}\end{array}$ \\
\hline Circulating leptin (P2-8) & $\begin{array}{l}\mathrm{M} \\
\mathrm{F}\end{array}$ & $\Downarrow$ & $\Downarrow$ & $\begin{array}{l}\checkmark \\
\checkmark\end{array}$ & $\begin{array}{l}5.6 \mathrm{a} \\
5.6 \mathrm{a}\end{array}$ \\
\hline Circulating leptin (P10-12) & $\begin{array}{l}\mathrm{M} \\
\mathrm{F}\end{array}$ & $\begin{array}{l}\Uparrow \\
\Uparrow\end{array}$ & . & & $\begin{array}{l}5.6 \mathrm{a} \\
5.6 \mathrm{a}\end{array}$ \\
\hline Rescue of POMC fibres by neonatal leptin & $\begin{array}{l}\mathrm{M} \\
\mathrm{F}\end{array}$ & 放 & . & & $\begin{array}{l}5.9 \mathrm{a} \\
5.9 \mathrm{a}\end{array}$ \\
\hline
\end{tabular}

Table 6.1: Concordance between BPA and DES animals

List of measures affected by either BPA $(0.02 \mathrm{ppm})$ or DES $(0.004 \mathrm{ppm})$ relative to same-sex, same-diet (if applicable) Control animals. 介 indicates measure is increased relative to Controls, $\Downarrow$ indicates measure is decreased relative to Controls. Instances of concordance between BPA and DES-treated animals are marked with $\checkmark$. Measures not affected by either BPA or DES are not included in this table. 
The other major instance of concordance involved the leptin surge. Up until P8 both BPA and DES animals were similar in their lack of the leptin surge observed in Control animals. On P10 and 12, the situation became more complicated, as BPAtreated animals showed a delayed leptin surge, while the DES animals remained perpetually at baseline levels. It is not known, however, what triggers this surge in leptin production in the first place. Leptin itself is supplied to neonates in the milk, but its concentration in the milk remains constant across the lactational days, so it is unlikely to be the source of the leptin surge $[333 ; 334 ; 335]$. The macronutrient content of milk does change over the days, and milk fat has been of particular interest given the role of dietary fat in stimulating leptin secretion. Milk fat is highest during the first few days of lactation [336; 337], and increased by maternal diet-induced obesity or high-fat diet exposure [338; 339]. Maternal food restriction markedly reduces milk production, but does not drastically affect milk composition-it may in fact increase fat content [340; 341]. Neither of these facts would seem to favour the production of a leptin surge, especially since by the time that surge normally begins, milk fat content has stabilized and remains fairly consistent thenceforth. More likely is a local regulation of leptin synthesis at the level of the adipose tissue. It could be that leptin synthesis is regulated by some other hormone that also happens to show a marked spike or trough at the time of the leptin surge: insulin, corticosterone, and thyroxine all meet this criteria [205], however it is not clear how they could be affected by either BPA or DES. Another possibility is that the leptin surge is set into motion by the action of sex steroids. If this were true, it would provide a substrate for the disruptive effects of the endocrine disruptors BPA and DES. However, several reasons exist why this is not likely to be the case. The sex steroids - though they show surges of their own during development - are out of phase with leptin, peaking either too early (testosterone) or too late (estradiol) to synchronize with the leptin surge [342]. Moreover, we have not found there to be any sex differences in either the timing or magnitude of the leptin surge, and this too seems to preclude a sex hormone-mediated origin. At any rate, correlations between postnatal leptin and other hormones do not imply directionality of causation, and even if they did, the issue of surge generation would simply be moved to another hormone and not actually solved in any great measure. The leptin surge may be a step in the adipogenic program triggered by some or another adipogenic transcription factor, and since BPA and DES both affect adipogenesis (albeit through different pathways, see Section 5.4), altered timing of leptin transcription may be one of many consequences. This prospect requires further investigation, but the discovery of compounds that affect the dynamics of the leptin surge may give future research a stronger purchase on the putative 'surge generator', as is often the case when a specific antagonist to some phenomenon of interest is found.

The most relevant discordances between our BPA- and DES-exposed animals concern the susceptibility to diet-induced obesity and glucose intolerance, because these 
measures are central to every other measure studied in this thesis. DES has long been suspected of having obesogenic properties, and while there are some studies that support this perspective [158; 159], this is not universal. Ryan et al., found, using methods nearly identical to ours, that DES at $0.004 \mathrm{ppm}$ was not particularly obesogenic in either males or females [143]. Yet our DES-exposed animals were comparable to BPA-exposed animals in terms of programmed leptin resistance, and if this alone were sufficient for the development of obesity, then the divergence here would be puzzling. More likely, in our view, is that since the leptin $\rightarrow$ POMC pathway was the only transcriptional pathway we found to be disrupted in our model, the numerous other outputs of leptin receptor signalling may have been sufficient to compensate for its absence. BPA-exposed animals, having reduced PVN innervation as an additional impairment may have been beyond the effective range of these hypothetical compensatory mechanisms.

In Chapter 5 we focused on the development of the POMC efferents to the PVN, finding them to be disrupted by BPA, but not DES exposure in several separate experiments. With the exception of Chapter 3 we measured this in absolute terms, making it possible to compare between the sexes. We generally found POMC staining intensity to be stronger in female Controls than in male animals, and the effect of BPA in these animals could be described as reducing it to male-like levels. The magnitude of reduction was smaller in male animals, though they had 'less to lose', in a sense. When we gave animals supplemental leptin injections during neonatal life, we found that the POMC fibres were rescued only in BPA-exposed females. Since in this regard females were relatively more impaired in the first place, the magnitude of recovery was correspondingly greater, and this may be the reason why we did not observe the same effect in males.

Reductions on POMC expression and fibre density similar to ours can be produced in females by early-life and adult exposure to dihydrotestosterone (DHT) [343; 238]. BPA is commonly regarded as an anti-androgen, but this may actually vary on a tissuespecific basis. For example, it was recently found that BPA alters the timing of neurogenesis in the zebrafish hypothalamus in a manner that can be blocked by AR antagonists [90]. If BPA's androgenic effects are tissue-specific, then an explanation for our observations involving the androgen receptor is not preposterous. An androgenic mechanism could also explain the absence of this effect in DES-exposed animals, as well as why some of our effects were a great deal stronger in female animals. If this were the case, the effects on neurite outgrowth would likely be cell autonomous, and thus not related to the altered leptin surge. In support of this is the fact that the leptin surge was disrupted in both BPA and DES animals, yet POMC efferents were unaffected in the latter. Comparatively little is known about the role of androgens in shaping the development of the hypothalamus, and what is known is complicated by interactions between the ERs, ARs, aromatase, and their effects on one another's ex- 
pression [342]. Further study of our model using an androgenic positive control, or with the inclusion of a group co-exposed to BPA and an antiandrogenic compound such as flutamide could be informative, but considerable effort would be needed to determine the appropriate compounds, doses, and timing.

BPA is a xenoestrogen by convention, but perhaps this is only because in 1936, estrogenic activity happened to be the first thing measured [51]. At the time it would have been impossible to describe BPA as 'PPARgenic', since that concept did not exist until the 1970s. It is also unlikely that BPA would have been described as a glucocorticoid mimic in 1936, since Selye's conception of stress was just introduced the very same year, and knowledge of adrenal steroids was minimal. Aside from that, researchers could have looked to see if BPA relieved rheumatoid arthritis, or if it prolonged survival post-adrenalectomy, but these are both a lot to ask of a low-dose endocrine disruptor. Even finding androgenic or anti-androgenic effects would have been difficult, given that testosterone had only been isolated in 1935 [344], and the methods for determining androgenicity were comparatively crude at the time. The fact that BPA is a demonstrably weak estrogen in many of the gold standard toxicological measures [345], yet still has a number of effects in studies that go beyond these measures suggests either that studies of the latter type (ours included) are somehow flawed, or that those of the former type are simply too narrow, beholden as they are to the classically defined actions of estrogens.

While many of our major findings can be slotted without much contortion into the category of estrogenic effects, this is not always the case. The organizational effects of estrogens on the measures we studied are not always sufficiently well characterized to allow the sorts of inferences needed to explain our observations. Additional research will improve this situation if not under its own momentum, then by the impetus of endocrine disruptor research, which yields a surfeit of disrupted systems to explain, but little insight into how those systems come about in the first place. At this stage it does seem certain that research on BPA will have to adopt a wider understanding of its nature in order to account for the diversity of its effects.

\subsection{IS BPA HAZARDOUS TO HUMANS?}

It has been our primary aim in these studies to model, as faithfully as possible, the conditions of human BPA exposure. To this end we used doses of BPA that are within the realm of environmental relevance and delivered orally to free-feeding animals. Accordingly, it would seem that our inferences are useful only to the extent that they can be applied to humans. To some extent, this perspective implies that the effects of endocrine disruptors on animals matter only insofar as those animals can be used as coal mine canaries for human health. Such anthropocentrism may not be warranted, after all BPA exposure is not limited to humans. BPA is ubiquitous in the environment and 
active as an endocrine disruptor in many diverse species including fish and amphibians [346; 347; 348], and—of course—rodents. For that matter, humans are not the only animals that have become heavier in recent decades. A parallel pattern of weight gain and obesity has been observed in a number of populations of wild, feral, and domestic animals living in close proximity to human populations [349]. It remains to be seen whether or not BPA affects animals at the ecological level, but such effects are certainly plausible and worthy of concern. Still, humans are the only animal species that voluntarily subjects itself to an environment that supports the development of obesity in so many other ways (i.e. the overabundance of high calorie food, sedentary lifestyles, and so forth), and so human interests predominate when proposing yet another obesogen to add to the mix.

Several attempts have been made to show that human health outcomes relate in some manner to circulating BPA. For example, a study of 3390 adults in China found a significant positive association between BPA exposure and abdominal obesity, as well as blood glucose concentration [350]. On the other hand, a study of urinary BPA concentration in patients with and without gestational diabetes mellitus failed to show any relationship between the two [351]. These examples, and for that matter all similar studies, have been cross-sectional in nature and plagued by technical limitations that curtail their interpretability [352]. There is some potential for following BPA exposure in prenatal and neonatal life, and using these data as the basis for a prospective study of some sort. BPA is detectable in maternal serum at the time of delivery, but this concentration is unrelated to birth weight, sex, or gestational duration [353]. Still, it would be possible to follow up on the subjects in this study and evaluate health outcomes in adulthood, after the effects of age and cumulative wear and tear have taken their toll. At the time of writing, no such study has been conducted.

An important consideration when dealing with questions of risk assessment concerns differences between human and animal development. Rodent models are commonly used in the study of brain and behavioural development, and the effects of sex and metabolic hormones on those processes. From these studies a great deal of progress has been made, and many new and unexpected mechanisms governing the sexual differentiation of the brain have been uncovered (for review, see [354]). However, humans and rodents differ to a degree that makes it imprudent to assume a great deal of mechanistic overlap, and thus it may be that anatomical, functional, and developmental effects observed in rodents are not present, or are of a completely different character in humans ${ }^{4}$. One key difference between humans and rodents concerns the effects of testosterone, which in rodents drives sexual differentiation of the brain in part through its aromatization to estradiol, but in humans acts directly on the brain as an an-

\footnotetext{
${ }^{4}$ Indeed, the process of sexual differentiation differs a great deal even between rats and mice, two species of rodents [355], to say nothing of differences between rats and humans.
} 
drogen [356]. We have argued that BPA's estrogenic and androgenic/anti-androgenic effects are highly relevant not only to the phenotype observed in our animals, but for its potential as an endocrine disruptor in general. This being the case, the differing roles for sex steroids in humans and rodents should form an important consideration in the risk assessment process. The importance of testosterone and its derived androgens in the masculinization process may provide a different substrate for BPA's effects, and the phenotypic outcomes of BPA exposure may differ correspondingly.

Critical periods during brain development do not necessary correspond between humans and rodents. Rodents are most sensitive to the organizational effects of sex steroids in the later embryonic and early postnatal periods, and it is during these times that circulating testosterone peaks in males [357]. Human males, in contrast, begin producing testosterone between the $2^{\text {nd }}$ and $3^{\text {rd }}$ trimesters of pregnancy, and this is when much of the masculinization process takes place [356]. Unconjugated BPA readily crosses the placental barrier and partitions into the milk, so exposure is possible throughout life in both humans and rodents (see Section 1.2), but human fetuses partake only in a fraction of the small quantities of BPA consumed by the mother that slip through first-pass metabolism during their critical period of sexual differentiation, and this may lessen its impact. This fact may also bear on concerns about the relatively higher levels of BPA observed in infants relative to adults. Non-human primates may someday be studied in order to clarify the situation, but at present research in that area is in its infancy. Indeed, the majority of studies into the effects of BPA on primates have been to clarify its pharmacokinetics (see Section 1.2), or its effects on peripheral tissues; brain development and sexual differentiation has not yet been a focus. Still, the presence of marked effects on rodents attests to the biological plausibility of BPA as an endocrine disruptor in humans, given that many of the receptors and enzymes with which it interacts are well-conserved between the species.

\subsubsection{Developmental Programming in Humans}

The murkiness of the relationship between BPA and human health precludes a definitive answer as to its health effects at this time. By following a less direct path, however, it may be possible to estimate the likelihood that the effects described in this thesis are relevant to humans. We have argued that early-life exposure to BPA programs body weight, food intake, metabolism, and most importantly, the neural substrates of those processes. The end result is an animal that is predisposed to obesity and its attendant metabolic consequences. Whether or not this can happen in humans is unknown at present, but it is possible at least to explore whether the mechanisms we propose to be affected by BPA exist in humans. If they do, then it becomes easier to justify treating our studies as a proof of concept for the effects of BPA on human health.

The phenomena we have observed may be subsumed under the category of develop- 
mental programming. Developmental programming has, of course, been well documented in a variety of animal models (see Section 2.3), and indeed the approach used in this thesis borrows heavily from that field. However, the concept itself originated in studies of human populations [358]. In 1977, Forsdahl gave an early demonstration of this phenomenon, showing that the infant mortality rate in the time and place an individual was born could be predictive of their likelihood of developing arteriosclerosis in adulthood [359]. Later, Hales and Barker were able to demonstrate a link between low birth weight and the risk of developing type II diabetes, hypertension, and insulin resistance in an unselected population sample [360;361]. Low birth weight is not the only variable that has been associated with the developmental programming of metabolic dysfunction: maternal type I diabetes, as well as gestational diabetes can have similar effects as well [362]. In both cases, the fetus is exposed to higher levels of glucose and insulin than would be expected in a metabolically healthy mother (Freinkel coined the phrase 'fuel-mediated teratogenesis' to refer to this state of affairs, since it was believed that glucose itself was a key mediator of these adverse outcomes [363]). A detailed analysis of neural circuitry and hormonal response in these human groups is unavailable at the time, but all that is needed to answer the question of applicability of this research to humans in a tentative way is evidence that humans are subject to developmental programming in some form. Research into this area is ongoing, and while the situation in humans is naturally a great deal more complex than the scenarios contrived for animal models, it is generally agreed that developmental programming exists in some form in humans, and that its study will provide a useful framework for understanding obesity and type II diabetes [358].

\subsubsection{Perinatal Leptin in Humans}

We have argued that some of our model's main phenotypic features can be best explained by alterations in the pattern of postnatal leptin secretion. Leptin, acting as it does in a neurotrophic capacity, must be secreted in appropriate amounts at developmentally appropriate times in order to see successful development of homeostatic circuitry. If it is true that BPA interferes with this, then it seems appropriate to ask whether leptin plays a similar role in human perinatal development. As in rats (see [364; 365]), maternal leptin concentration reliably increases over the gestational period $[366 ; 367 ; 368]$. The human placenta produces leptin that is measurable at the time of birth, though it appears that only $5 \%$ of this is delivered to the fetal side of circulation $[369 ; 370]$. For its part, the fetus produces leptin in preadipocytes as early as the $6^{\text {th }}$ week of life, and continues to do so as mature adipose tissue develops [371;369]. Fetal leptin levels begin to climb rapidly beginning in the $35^{\text {th }}$ week of prenatal life, keeping pace with the rapid increase in fetal weight that occurs in late gestation [372]. Fetal leptin is positively correlated with body weight, ponderal index, and body mass index in 
growth-restricted and average sized human fetuses [373; 374]. Leptin levels in the umbilical cord blood are similarly correlated with body weight and the various measures of composition $[375 ; 376 ; 369 ; 377 ; 366]$. Placental and cord blood leptin is also elevated in the newborns of diabetic mothers [376]. Leptin is also present in the breast milk, showing a positive correlation with maternal plasma leptin, and a negative correlation with fetal body weight and the rate of weight gain during neonatal life [378; 379; 380]. There can be little doubt, therefore, that humans are exposed to leptin during the perinatal period, and importantly that the degree of exposure seems to be correlated with various measures of growth and vitality (for a recent review, see [381]). Of course, experimental verification is needed to confirm the causal link between perinatal leptin and developmental programming already known to exist in rodents, but experiments like this are unlikely to take place without further development and maturation of the field.

\subsection{CONCLUSION}

Human energy homeostasis is susceptible to developmental programming through exposure to adverse nutritional environments, and rodent models are now in use that capture and model this phenomenon. Through these efforts, a number of distinguishing behavioural and neurobiological markers have emerged. We have now shown that many of these same markers, discovered originally in service of modelling human nutritional programming, are also affected by exposure to environmentally relevant levels of endocrine disrupting compounds. One of our proposed effectors is leptin, and since it acts similarly during the perinatal period in both humans and rodents, the possibility of it being affected by environmental exposure to BPA in humans is not out of the question. In answering the question of human health, there are relevant pharmacokinetic differences that would come to bear (see Section 1.2), but the only requirement that cannot be circumvented is that BPA be somehow able to reach the fetus or newbornand it does. With this in mind, and taking into account our findings as well as those of others in the field, many of which directly model measurable human clinical outcomes, there is sufficient basis for concern about the effects of human BPA exposure. 
Appendices 


\section{Appendix A}

\section{Details on Experimental Methods}

\section{A.1 DETAILED DIET COMPOSITION}

\begin{tabular}{|c|c|c|c|c|c|c|c|c|c|c|}
\hline & \multicolumn{2}{|c|}{ Control (AIN93G) } & \multicolumn{2}{|c|}{0.004 ppm DES } & \multicolumn{2}{|c|}{0.001 ppm BPA } & \multicolumn{2}{|c|}{$0.02 \mathrm{ppm} \mathrm{BPA}$} & \multicolumn{2}{|c|}{ High-fat diet } \\
\hline & $g^{o} \%$ & $k C a l \%$ & $g \%$ & $\mathrm{kCal} \%$ & $g \%$ & $\mathrm{kCal} \%$ & $g^{0} \%$ & kCal\% & $g \%$ & $k C a{ }^{1} \%$ \\
\hline Protein & 20 & 20 & 20 & 20 & 20 & 20 & 20 & 20 & 26.2 & 20 \\
\hline Carbohydrate & 64 & 64 & 64 & 64 & 64 & 64 & 64 & 64 & 26.3 & 20 \\
\hline Fat & 7 & 16 & 7 & 16 & 7 & 16 & 7 & 16 & 34.9 & 60 \\
\hline Total & & 100 & & 100 & & 100 & & 100 & & 100 \\
\hline $\mathrm{kCal} / \mathrm{g}$ & 4 & & 4 & & 4 & & 4 & & 5.24 & \\
\hline Ingredient & $g$ & kCal & $g$ & $k C a l$ & $g$ & kCal & $g$ & kCal & $g$ & $k$ Cal \\
\hline Casein & 200 & 800 & 200 & 800 & 200 & 800 & 200 & 800 & 200 & 800 \\
\hline L-Cystine & 3 & 12 & 3 & 12 & 3 & 12 & 3 & 12 & 3 & 12 \\
\hline Corn starch & 397.486 & 1590 & 397.486 & 1590 & 397.486 & 1590 & 397.486 & 1590 & 0 & 0 \\
\hline Maltodextrin & 132 & 528 & 132 & 528 & 132 & 528 & 132 & 528 & 125 & 500 \\
\hline Sucrose & 100 & 400 & 100 & 400 & 100 & 400 & 100 & 400 & 68.8 & 275.2 \\
\hline Cellulose BW200 & 50 & 0 & 50 & 0 & 50 & 0 & 50 & 0 & 50 & 0 \\
\hline Soybean oil & 70 & 630 & 70 & 630 & 70 & 630 & 70 & 630 & 25 & 225 \\
\hline Diethylstilbestrol (DES) & 0 & 0 & 0.000004 & 0 & 0 & 0 & 0 & 0 & 0 & 0 \\
\hline Bisphenol A (BPA) & 0 & 0 & 0 & 0 & 0.000001 & 0 & 0.00002 & 0 & 0 & 0 \\
\hline t-Butylhydroquinone & 0.014 & 0 & 0.014 & 0 & 0.014 & 0 & 0.014 & 0 & 0.014 & 0 \\
\hline Mineral mix S10022G & 35 & 0 & 35 & 0 & 35 & 0 & 35 & 0 & 0 & 0 \\
\hline Mineral mix S10026 & 0 & 0 & 0 & 0 & 0 & 0 & 0 & 0 & 10 & 0 \\
\hline Vitamin mix V10037 & 10 & 40 & 10 & 40 & 10 & 40 & 10 & 40 & 0 & 0 \\
\hline Vitamin mix V10001 & 0 & 0 & 0 & 0 & 0 & 0 & 0 & 0 & 10 & 40 \\
\hline Dicalcium phosphate & 0 & 0 & 0 & 0 & 0 & 0 & 0 & 0 & 13 & 0 \\
\hline Calcium carbonate & 0 & 0 & 0 & 0 & 0 & 0 & 0 & 0 & 5.5 & 0 \\
\hline Potassium citrate & 0 & 0 & 0 & 0 & 0 & 0 & 0 & 0 & 16.5 & 0 \\
\hline Choline bitartrate & 2.5 & 0 & 2.5 & 0 & 2.5 & 0 & 2.5 & 0 & 2 & 0 \\
\hline Yellow Dye 5 & 0 & 0 & 0.05 & 0 & 0 & 0 & 0 & 0 & 0 & 0 \\
\hline Red Dye 40 & 0 & 0 & 0 & 0 & 0.05 & 0 & 0 & 0 & 0 & 0 \\
\hline Blue Dye 1 & 0 & 0 & 0 & 0 & 0 & 0 & 0.05 & 0 & 0.05 & 0 \\
\hline Total & 1000 & 4000 & 1000 & 4000 & 1000 & 4000 & 1000 & 4000 & 773.85 & 4057 \\
\hline
\end{tabular}

Table A.1: Composition of diets used in these studies. 


\section{A.2 PRIMERS FOR QRT-PCR}

\begin{tabular}{lll}
\hline Name & Forward $\left(5^{\prime}-3^{\prime}\right)$ & Reverse $\left(5^{\prime}-3^{\prime}\right)$ \\
\hline ER $\alpha$ & ACCATTGACAAGAACCGGAG & CCTGAAGCACCCATTTCATT \\
AgRP & CGGAGGTGCTAGATCCACAGA & AGGACTCGTGCAGCCTACAC \\
NPY & TACCCTCCAAGCCGGACAA & TTTCATTTCCCATCACCACATG \\
POMC & GCCCTCCTGCTTCAGACCTC & CGTTGCCAGGAAACACGG \\
ObRB & CCACTGTTGCTTTGGGAATG & GTCCCCTTCATCCAGCA \\
FOXO1 & GCTGCAATGGCTATGGTAGGA & GTCACAGTCCAAGCGCTCAAT \\
SOCS3 & GCGGGCACCTTTCTTATCC & TCCCCGACTGGGTCTTGAC \\
STAT3 & GGGGATGTTGCTGCCCTCAG & GGCACGGCCTCCATTCCCACAT \\
Leptin & ATTTCACACACGTCGGTAT & AAGCCCAGGAATGAAGTCA \\
GAPDH & GCAGTGGCAAAGTGGAGATTGTTGC & CCCGTTGATGACAAGCTTCCCATTC \\
$\beta$-Actin & GAACCCTAAGGCCAACCGTG & GGTACGACCAGAGGCATACAG \\
RPS18 & TTCAGCACATCCTGCGAGTA & TTGGTGAGGTCAATGTCTGC \\
\hline
\end{tabular}

Table A.2: Primer sequences for qRT-PCR. 


\section{Appendix B}

\section{Copyright Documentation}

\section{B.1 PERMISSION FROM ENDOCRINOLOGY}

Endocrinology

ISSN: 1945-717

Publication Type: e-Journal

Volume:154

Issue:4

Start page: 1465

Publisher: ENDOCRINE SOCIETY Author/Editor: Endocrine Society; HighWire Press

Permission type: Republish or display content

Type of use: Republish in a thesis/dissertation

Permission Status: $\checkmark$ Granted 


\title{
B.2 CO-AUTHOR PERMISSION STATEMENTS
}

\author{
To whom it may concern, \\ I, Zack Patterson, hereby grant permission to Harry MacKay to reproduce \\ all content associated with Organizational Effects of Perinatal Exposure to \\ Bisphenol-A and Diethylstilbestrol on Arcuate Nucleus Circuitry Control- \\ ling Food Intake and Energy Expenditure in Male and Female CD-1 Mice \\ published in Endocrinology in April 2013. \\ I am also aware that the author of this thesis will be granted non-exclusive li- \\ censes to Carleton University Library and the Library and Archives Canada. \\ Licenses are available at: \\ Carleton University Library: \\ http://www2.carleton.ca/fgpa/ccms/wp-content/ccms-files/Licence-to-Carleton.pdf \\ Library and Archives Canada: \\ http://www.collectionscanada.gc.ca/obj/s4/f2/frm-nl59-2.pdf \\ Signature:

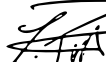 \\ Name in print: Zack Patterson \\ Date: $\quad$ March 28th, 2015
}


To whom it may concern,

I, Rim Khazall, hereby grant permission to Harry MacKay to reproduce all content associated with Organizational Effects of Perinatal Exposure to Bisphenol-A and Diethylstilbestrol on Arcuate Nucleus Circuitry Controlling Food Intake and Energy Expenditure in Male and Female CD-1 Mice published in Endocrinology in April 2013.

I am also aware that the author of this thesis will be granted non-exclusive licenses to Carleton University Library and the Library and Archives Canada. Licenses are available at:

Carleton University Library:

http://www2.carleton.ca/fgpa/ccms/wp-content/ccms-files/Licence-to-Carleton.pdf

Library and Archives Canada:

http://www.collectionscanada.gc.ca/obj/s4/f2/frm-nl59-2.pdf

Signature:

March, 30, 2015 
To whom it may concern,

I, Shoyeb Patel, hereby grant permission to Harry MacKay to reproduce all content associated with Organizational Effects of Perinatal Exposure to Bisphenol-A and Diethylstilbestrol on Arcuate Nucleus Circuitry Controlling Food Intake and Energy Expenditure in Male and Female CD-1 Mice published in Endocrinology in April 2013.

I am also aware that the author of this thesis will be granted non-exclusive licenses to Carleton University Library and the Library and Archives Canada. Licenses are available at:

Carleton University Library:

http://www2.carleton.ca/fgpa/ccms/wp-content/ccms-files/Licence-to-Carleton.pdf

Library and Archives Canada:

http://www.collectionscanada.gc.ca/obj/s4/f2/frm-nl59-2.pdf

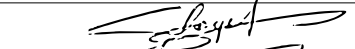

Signature:

Name in print: SHOYEB PATEL

Date: Jan 20, 2015 
To whose it may concane,

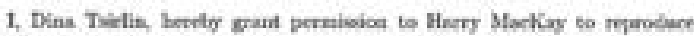

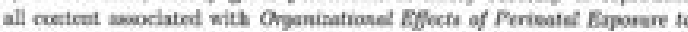

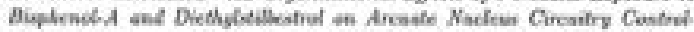

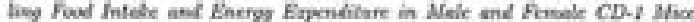
pulilistod in Endacrineleys is Apral 3013

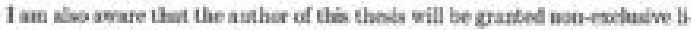

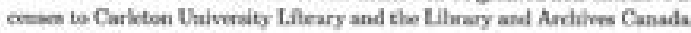
Isomates are acailable at

Carleton Undventity Liteary:

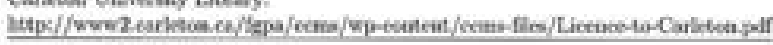

Lllorary and Anctives Canade

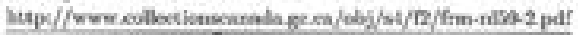

(7)

Signature:

(1)

Name in print: Dinn Tsoritin

Date Jan 20/2015 
To whom it may concern,

I, Alfonso Abizaid, hereby grant permission to Harry MacKay to reproduce all content associated with Organizational Effects of Perinatal Exposure to Bisphenol-A and Diethylstilbestrol on Arcuate Nucleus Circuitry Controlling Food Intake and Energy Expenditure in Male and Female CD-1 Mice published in Endocrinology in April 2013.

I am also aware that the author of this thesis will be granted non-exclusive licenses to Carleton University Library and the Library and Archives Canada. Licenses are available at:

Carleton University Library:

http://www2.carleton.ca/fgpa/ccms/wp-content/ccms-files/Licence-to-Carleton.pdf

Library and Archives Canada:

http://www.collectionscanada.gc.ca/obj/s4/f2/frm-nl59-2.pdf

Signature:

Name in print: Alfonso Abizaid

Date: March 30, 2015 


\section{Bibliography}

[1] Delfosse, V., Grimaldi, M., le Maire, A., Bourguet, W., and Balaguer, P. (2014) Nuclear receptor profiling of bisphenol-A and its halogenated analogues., vol. 94. Elsevier Inc.

[2] Eladak, S., Grisin, T., Moison, D., Guerquin, M.-J., N’Tumba-Byn, T., PozziGaudin, S., Benachi, A., Livera, G., Rouiller-Fabre, V., and Habert, R. (2014) A new chapter in the bisphenol A story: bisphenol $\mathrm{S}$ and bisphenol $\mathrm{F}$ are not safe alternatives to this compound. Fertility and sterility, pp. 11-21.

[3] Vandenberg, L. N., Hauser, R., Marcus, M., Olea, N., and Welshons, W. V. (2007) Human exposure to bisphenol A (BPA). Reproductive toxicology, 24, 139-77.

[4] Hoekstra, E. J. and Simoneau, C. (2013) Release of bisphenol A from polycarbonate: a review. Critical reviews in food science and nutrition, 53, 386-402.

[5] Krishnan, A. and Stathis, P. (1993) Bisphenol-A: An Estrogenic Substance is Released from Polycarbonate Flasks during Autoclaving. Endocrinology.

[6] Maragou, N. C., Makri, A., Lampi, E. N., Thomaidis, N. S., and Koupparis, M. a. (2008) Migration of bisphenol A from polycarbonate baby bottles under real use conditions. Food additives \& contaminants. Part A, Chemistry, analysis, control, exposure \& risk assessment, 25, 373-83.

[7] Nam, S.-H., Seo, Y.-M., and Kim, M.-G. (2010) Bisphenol A migration from polycarbonate baby bottle with repeated use. Chemosphere, 79, 949-52.

[8] Biedermann-Brem, S., Grob, K., and Fjeldal, P. (2008) Release of bisphenol A from polycarbonate baby bottles: mechanisms of formation and investigation of worst case scenarios. European Food Research and Technology, 227, 1053-1060.

[9] Teeguarden, J. G., Calafat, A. M., Ye, X., Doerge, D. R., Churchwell, M. I., Gunawan, R., and Graham, M. K. (2011) Twenty-four hour human urine and serum profiles of bisphenol a during high-dietary exposure. Toxicological sciences, $123,48-57$. 
[10] Christensen, K. L. Y., Lorber, M., Koch, H. M., Kolossa-Gehring, M., and Morgan, M. K. (2012) Population variability of phthalate metabolites and bisphenol A concentrations in spot urine samples versus $24-$ or 48 -h collections. Journal of exposure science \& environmental epidemiology, 22, 632-40.

[11] Lakind, J. S., Levesque, J., Dumas, P., Bryan, S., Clarke, J., and Naiman, D. Q. (2012) Comparing United States and Canadian population exposures from $\mathrm{Na}$ tional Biomonitoring Surveys: bisphenol A intake as a case study. Journal of exposure science \& environmental epidemiology, 22, 219-26.

[12] (2012) Updated Assessment of Bisphenol A (BPA) Exposure from Food Sources. Tech. Rep. September.

[13] Vom Saal, F. S., Prins, G. S., and Welshons, W. V. (2012) Report of very low real-world exposure to bisphenol A is unwarranted based on a lack of data and flawed assumptions. Toxicological sciences, 125, 318-20.

[14] (2008) Health Risk Assessment of Bisphenol A from Food Packaging Applications. Tech. rep.

[15] (2006) Opinion of the Scientific Panel on Food Additives, Flavourings, Processing Aids and Materials in Contact with Food. Tech. rep.

[16] Christensen, K. L. Y., Lorber, M., Koslitz, S., Brüning, T., and Koch, H. M. (2012) The contribution of diet to total bisphenol A body burden in humans: results of a 48 hour fasting study. Environment international, 50, 7-14.

[17] Upmeier, A., Degen, G. H., Diel, P., Michna, H., and Bolt, H. M. (2000) Toxicokinetics of bisphenol A in female DA/Han rats after a single i.v. and oral administration. Archives of Toxicology, 74, 431-436.

[18] Pottenger, L. H., Domoradzki, J. Y., Markham, D. a., Hansen, S. C., Cagen, S. Z., and Waechter, J. M. (2000) The relative bioavailability and metabolism of bisphenol A in rats is dependent upon the route of administration. Toxicological sciences, 54, 3-18.

[19] Yokota, H., Iwano, H., Endo, M., Kobayashi, T., Inoue, H., and Ikushiro, S. (1999) Glucuronidation of the environmental estrogen bisphenol A by an isoform of UDP-glucuronosyltransferase, UGT2B1, in the rat liver. Biochem. J, 409, 405-409.

[20] Inoue, H., Yuki, G., Yokota, H., and Kato, S. (2003) Bisphenol A glucuronidation and absorption in rat intestine. Drug Metabolism and Disposition, 31, 140-144. 
[21] Matthews, J., Twomey, K., and Zacharewski, T. (2001) In Vitro and in Vivo Interactions of Bisphenol A and Its Metabolic, Bisphenol A Glucuronide, with Estrogen Receptors $\alpha$ and $\beta$. Chemical research in toxicology, 14.

[22] Snyder, R. W., Maness, S. C., Gaido, K. W., Welsch, F., Sumner, S. C., and Fennell, T. R. (2000) Metabolism and disposition of bisphenol A in female rats. Toxicology and applied pharmacology, 168, 225-34.

[23] Inoue, H., Yokota, H., Makino, T., Yuasa, A., and Kato, S. (2001) Bisphenol A glucuronide, a major metabolite in rat bile after liver perfusion. Drug Metabolism and Disposition, 29, 1084-1087.

[24] Sakamoto, H., Yokota, H., Kibe, R., Sayama, Y., and Yuasa, A. (2002) Excretion of bisphenol A-glucuronide into the small intestine and deconjugation in the cecum of the rat. Biochimica et biophysica acta, 1573, 171-6.

[25] Völkel, W., Colnot, T., Csanády, G. a., Filser, J. G., and Dekant, W. (2002) Metabolism and kinetics of bisphenol a in humans at low doses following oral administration. Chemical research in toxicology, 15, 1281-7.

[26] Ginsberg, G. and Rice, D. C. (2009) Does rapid metabolism ensure negligible risk from bisphenol A? Environmental health perspectives, 117, 1639-43.

[27] Nishikawa, M., Iwano, H., Yanagisawa, R., Koike, N., Inoue, H., and Yokota, H. (2010) Placental transfer of conjugated bisphenol A and subsequent reactivation in the rat fetus. Environmental health perspectives, 118, 1196-203.

[28] Matsumoto, J., Yokota, H., and Yuasa, A. (2002) Developmental increases in rat hepatic microsomal UDP-glucuronosyltransferase activities toward xenoestrogens and decreases during pregnancy. Environmental Health Perspectives, 110, 193-196.

[29] Takeuchi, T., Tsutsumi, O., Nakamura, N., Ikezuki, Y., Takai, Y., Yano, T., and Taketani, Y. (2004) Gender difference in serum bisphenol A levels may be caused by liver UDP-glucuronosyltransferase activity in rats. Biochemical and biophysical research communications, 325, 549-54.

[30] Inoue, H., Tsuruta, A., Kudo, S., and Ishii, T. (2005) Bisphenol A glucuronidation and excretion in liver of pregnant and nonpregnant female rats. Drug Metabolism and Disposition, 33, 55-59.

[31] Miyakoda, H., Tabata, M., Onodera, S., and Takeda, K. (1999) Passage of bisphenol A into the fetus of the pregnant rat. Journal of Health Science. 
[32] Shin, B. S., Yoo, S. D., Cho, C. Y., Jung, J. H., Lee, B. M., Kim, J. H., Lee, K. C., Han, S.-Y., Kim, H. S., and Park, K. L. (2002) Maternal-fetal disposition of bisphenol a in pregnant Sprague-Dawley rats. Journal of toxicology and environmental health, 65, 395-406.

[33] Balakrishnan, B., Henare, K., Thorstensen, E. B., Ponnampalam, A. P., and Mitchell, M. D. (2010) Transfer of bisphenol A across the human placenta. American journal of obstetrics and gynecology, 202, 393.e1-7.

[34] Mø rck, T. J., Sorda, G., Bechi, N., Rasmussen, B. S., Nielsen, J. B., Ietta, F., Rytting, E., Mathiesen, L., Paulesu, L., and Knudsen, L. E. (2010) Placental transport and in vitro effects of Bisphenol A. Reproductive toxicology, 30, 131-7.

[35] Tanaka, M., Kawamoto, T., and Matsumoto, H. (2010) Distribution of 14Cbisphenol A in pregnant and newborn mice. Dental materials, 26, 181-187.

[36] Kurebayashi, H., Nagatsuka, S.-I., Nemoto, H., Noguchi, H., and Ohno, Y. (2005) Disposition of low doses of 14C-bisphenol A in male, female, pregnant, fetal, and neonatal rats. Archives of toxicology, 79, 243-52.

[37] Okabayashi, K. and Watanabe, T. (2010) Excretion of bisphenol A into rat milk. Toxicology mechanisms and methods, 20, 133-6.

[38] Doerge, D. R., Vanlandingham, M., Twaddle, N. C., and Delclos, K. B. (2010) Lactational transfer of bisphenol A in Sprague-Dawley rats. Toxicology letters, 199, 372-6.

[39] Yoo, S. D., Shin, B. S., Lee, B. M., Lee, K. C., Han, S. Y., Kim, H. S., Kwack, S. J., and Park, K. L. (2001) Bioavailability and mammary excretion of bisphenol a in Sprague-Dawley rats. Journal of toxicology and environmental health, 64, 417-426.

[40] Reif, D. M., Martin, M. T., Tan, S. W., Houck, K. a., Judson, R. S., Richard, A. M., Knudsen, T. B., Dix, D. J., and Kavlock, R. J. (2010) Endocrine profiling and prioritization of environmental chemicals using ToxCast data. Environmental health perspectives, 118, 1714-20.

[41] Vom Saal, F. S., Nagel, S. C., Coe, B. L., Angle, B. M., and Taylor, J. a. (2012) The estrogenic endocrine disrupting chemical bisphenol A (BPA) and obesity. Molecular and cellular endocrinology, 354, 74-84.

[42] Blair, R. M., Fang, H., Branham, W. S., Hass, B. S., Dial, S. L., Moland, C. L., Tong, W., Shi, L., Perkins, R., and Sheehan, D. M. (2000) The estrogen receptor relative binding affinities of 188 natural and xenochemicals: structural diversity of ligands. Toxicological sciences, 54, 138-153. 
[43] Glascock, R. F. and Hoekstra, W. G. (1959) Selective accumulation of tritiumlabelled hexoestrol by the reproductive organs of immature female goats and sheep. The Biochemical journal, 72, 673-82.

[44] Jensen, E. and DeSombre, E. (1973) Estrogen-Receptor Interaction. Science, 182, $126-134$.

[45] Greene, G., Gilna, P., Waterfield, M., and Baker, A. (1986) Sequence and expression of human estrogen receptor complementary DNA. Science, 231, 1150-1154.

[46] Kuiper, G. and Enmark, E. (1996) Cloning of a novel estrogen receptor expressed in rat prostate and ovary. Proceedings of the National Academy of Sciences, 93, 5925-5930.

[47] Lubahn, D. and Moyer, J. (1993) Alteration of reproductive function but not prenatal sexual development after insertional disruption of the mouse estrogen receptor gene. Proceedings of the National Academy of Sciences, 90, 11162-11166.

[48] Krege, J. and Hodgin, J. (1998) Generation and reproductive phenotypes of mice lacking estrogen receptor beta. Proceedings of the National Academy of Sciences, 95, 15677-15682.

[49] Heldring, N., et al. (2007) Estrogen receptors: how do they signal and what are their targets. Physiological reviews, 87, 905-31.

[50] Delfosse, V. and Grimaldi, M. (2012) Structural and mechanistic insights into bisphenols action provide guidelines for risk assessment and discovery of bisphenol A substitutes. Proceedings of the National Academy of Sciences.

[51] Dodds, E. and Lawson, W. (1936) Synthetic oestrogenic agents without the phenanthrene nucleus. Nature.

[52] Hiroi, H., Tsutsumi, O., and Momoeda, M. (1999) Differential interactions of bisphenol A and 17beta-estradiol with estrogen receptor alpha (ERalpha) and ERbeta. Endocrine Journal, 46, 773-778.

[53] Kurosawa, T., Hiroi, H., and Tsutsumi, O. (2002) The Activity of Bisphenol A Depends on Both the Estrogen Receptor Subtype and the Cell Type. Endocrine Journal, 49, 465-471.

[54] Gould, J. C., Leonard, L. S., Maness, S. C., Wagner, B. L., Conner, K., Zacharewski, T., Safe, S., McDonnell, D. P., and Gaido, K. W. (1998) Bisphenol A interacts with the estrogen receptor alpha in a distinct manner from estradiol. Molecular and cellular endocrinology, 142, 203-14. 
[55] Gronemeyer, H., Gustafsson, J.-A., and Laudet, V. (2004) Principles for modulation of the nuclear receptor superfamily. Nature reviews: Drug discovery, 3, 950-64.

[56] Smith, A., Bosch, M., Wagner, E. J., Rø nnekleiv, O., and Kelly, M. (2013) The membrane estrogen receptor ligand STX rapidly enhances GABAergic signaling in NPY/AgRP neurons: role in mediating the anorexigenic effects of $17 \beta$ estradiol. American journal of physiology. Endocrinology and metabolism, 305, E632-40.

[57] Chambliss, K. and Wu, Q. (2010) Non-nuclear estrogen receptor $\alpha$ signaling promotes cardiovascular protection but not uterine or breast cancer growth in mice. The Journal of clinical investigation, 120.

[58] Marino, M., Pellegrini, M., La Rosa, P., and Acconcia, F. (2012) Susceptibility of estrogen receptor rapid responses to xenoestrogens: Physiological outcomes. Steroids, 77, 910-7.

[59] Srivastava, D. P., Waters, E. M., Mermelstein, P. G., Kramár, E. a., Shors, T. J., and Liu, F. (2011) Rapid estrogen signaling in the brain: implications for the fine-tuning of neuronal circuitry. The Journal of neuroscience, 31, 16056-63.

[60] Kelly, M. J. and Rø nnekleiv, O. K. (2012) Membrane-initiated actions of estradiol that regulate reproduction, energy balance and body temperature. Frontiers in neuroendocrinology, 33, 376-87.

[61] Micevych, P. and Dominguez, R. (2009) Membrane estradiol signaling in the brain. Frontiers in neuroendocrinology, 30, 315-27.

[62] Fiocchetti, M., Ascenzi, P., and Marino, M. (2012) Neuroprotective effects of $17 \beta$-estradiol rely on estrogen receptor membrane initiated signals. Frontiers in physiology, 3, 73 .

[63] Marino, M. and Ascenzi, P. (2006) Steroid hormone rapid signaling: the pivotal role of S-palmitoylation. IUBMB life, 58, 716-9.

[64] Meitzen, J., Luoma, J. I., Boulware, M. I., Hedges, V. L., Peterson, B. M., Tuomela, K., Britson, K. a., and Mermelstein, P. G. (2013) Palmitoylation of estrogen receptors is essential for neuronal membrane signaling. Endocrinology, 154, 4293-4304.

[65] Bolli, A., Galluzzo, P., Ascenzi, P., Del Pozzo, G., Manco, I., Vietri, M. T., Mita, L., Altucci, L., Mita, D. G., and Marino, M. (2008) Laccase treatment impairs bisphenol A-induced cancer cell proliferation affecting estrogen receptor alphadependent rapid signals. IUBMB life, 60, 843-52. 
[66] Hong, H., Yang, L., and Stallcup, M. R. (1999) Hormone-independent Transcriptional Activation and Coactivator Binding by Novel Orphan Nuclear Receptor ERR3. Journal of Biological Chemistry, 274, 22618-22626.

[67] Takayanagi, S., Tokunaga, T., Liu, X., Okada, H., Matsushima, A., and Shimohigashi, Y. (2006) Endocrine disruptor bisphenol A strongly binds to human estrogen-related receptor gamma (ERRgamma) with high constitutive activity. Toxicology letters, 167, 95-105.

[68] Okada, H., Tokunaga, T., Liu, X., Takayanagi, S., Matsushima, A., and Shimohigashi, Y. (2008) Direct evidence revealing structural elements essential for the high binding ability of bisphenol A to human estrogen-related receptor-gamma. Environmental health perspectives, 116, 32-8.

[69] Tohmé, M., Prud'homme, S. M., Boulahtouf, A., Samarut, E., Brunet, F., Bernard, L., Bourguet, W., Gibert, Y., Balaguer, P., and Laudet, V. (2014) Estrogen-related receptor $\gamma$ is an in vivo receptor of bisphenol A. FASEB journal, 28, 3124-33.

[70] Liu, X., Matsushima, A., Shimohigashi, M., and Shimohigashi, Y. (2014) A characteristic back support structure in the bisphenol A-binding pocket in the human nuclear receptor ERR $\gamma$. PloS one, 9, e101252.

[71] Montes-Grajales, D. and Olivero-Verbel, J. (2013) Computer-aided identification of novel protein targets of bisphenol A. Toxicology letters, 222, 312-20.

[72] Prossnitz, E. R., Arterburn, J. B., Smith, H. O., Oprea, T. I., Sklar, L. a., and Hathaway, H. J. (2008) Estrogen signaling through the transmembrane G protein-coupled receptor GPR30. Annual review of physiology, 70, 165-90.

[73] Revankar, C. M., Cimino, D. F., Sklar, L. a., Arterburn, J. B., and Prossnitz, E. R. (2005) A transmembrane intracellular estrogen receptor mediates rapid cell signaling. Science, 307, 1625-30.

[74] Filardo, E. J., Quinn, J. a., Frackelton, a. R., and Bland, K. I. (2002) Estrogen action via the $G$ protein-coupled receptor, GPR30: stimulation of adenylyl cyclase and cAMP-mediated attenuation of the epidermal growth factor receptor-toMAPK signaling axis. Molecular endocrinology, 16, 70-84.

[75] Thomas, P. and Dong, J. (2006) Binding and activation of the seventransmembrane estrogen receptor GPR30 by environmental estrogens: a potential novel mechanism of endocrine disruption. The Journal of steroid biochemistry and molecular biology, 102, 175-9. 
[76] Dong, S., Terasaka, S., and Kiyama, R. (2011) Bisphenol A induces a rapid activation of Erk1/2 through GPR30 in human breast cancer cells. Environmental pollution, 159, 212-8.

[77] Chung, E., Genco, M. C., Megrelis, L., and Ruderman, J. V. (2011) Effects of bisphenol A and triclocarban on brain-specific expression of aromatase in early zebrafish embryos. Proceedings of the National Academy of Sciences of the United States of America, 108, 17732-7.

[78] Pearlman, W. H. and Pearlman, M. R. (1961) The metabolism in vivo of Delta 4-androstene-3, 17-dione-7-H3; its localization in the ventral prostate and other tissues of the rat. The Journal of biological chemistry, 236, 1321-7.

[79] Fang, S., Anderson, K., and Liao, S. (1969) Receptor Proteins for Androgens. Journal of Biological Chemistry, 244.

[80] Bruchovsky, N. and Wilson, J. (1968) The intranuclear binding of testosterone and $5 \alpha$-androstan-17 $\beta$-ol-3-one by rat prostate. Journal of Biological Chemistry.

[81] Gustafasson, J. and Pousette, K. (1975) Demonstration and partial characterization of cytosol receptors for testosterone. Biochemistry, 14, 3094-101.

[82] Lubahn, D., Joseph, D., and Sullivan, P. (1988) Cloning of Human Androgen Rector Complentry DNA and Localization to the X Chromosome. Science, pp. $327-330$.

[83] Tan, M. E., Li, J., Xu, H. E., Melcher, K., and Yong, E.-L. (2014) Androgen receptor: structure, role in prostate cancer and drug discovery. Acta pharmacologica Sinica, pp. 1-21.

[84] Lee, H. J., Chattopadhyay, S., Gong, E.-Y., Ahn, R. S., and Lee, K. (2003) Antiandrogenic effects of bisphenol A and nonylphenol on the function of androgen receptor. Toxicological sciences, 75, 40-6.

[85] Teng, C., Goodwin, B., Shockley, K., Xia, M., Huang, R., Norris, J., Alex Merrick, B., Jetten, A. M., Austin, C. P., and Tice, R. R. (2013) Bisphenol A affects androgen receptor function via multiple mechanisms. Chemico-biological interactions, pp. 1-9.

[86] Sun, H., Xu, L.-C., Chen, J.-F., Song, L., and Wang, X.-R. (2006) Effect of bisphenol A, tetrachlorobisphenol A and pentachlorophenol on the transcriptional activities of androgen receptor-mediated reporter gene. Food and chemical toxicology, 44, 1916-21. 
[87] Li, J., Ma, M., and Wang, Z. (2010) In vitro profiling of endocrine disrupting effects of phenols. Toxicology in vitro, 24, 201-7.

[88] Fang, H., Tong, W., Branham, W. S., Moland, C. L., Dial, S. L., Hong, H., Xie, Q., Perkins, R., Owens, W., and Sheehan, D. M. (2003) Study of 202 natural, synthetic, and environmental chemicals for binding to the androgen receptor. Chemical research in toxicology, 16, 1338-58.

[89] Paris, F., Balaguer, P., Térouanne, B., Servant, N., Lacoste, C., Cravedi, J.-P., Nicolas, J.-c., and Sultan, C. (2002) Phenylphenols, biphenols, bisphenol-A and 4-tert- octylphenol exhibit alpha and beta estrogen activities and antiandrogen activity in reporter cell lines. Molecular and cellular endocrinology, 193, 43-9.

[90] Kinch, C. D., Ibhazehiebo, K., Jeong, J.-H., Habibi, H. R., and Kurrasch, D. M. (2015) Low-dose exposure to bisphenol A and replacement bisphenol S induces precocious hypothalamic neurogenesis in embryonic zebrafish. Proceedings of the National Academy of Sciences, p. 201417731.

[91] Green, H. and Kehinde, O. (1975) An established preadipose cell line and its differentiation in culture. II. Factors affecting the adipose conversion. Cell, 5, $19-27$.

[92] Lalwani, N., Fahl, W., and Reddy, J. (1983) Detection of a nafenopin-binding protein in rat liver cytosol associated with the induction of peroxisome proliferation by hypolipidemic compounds. Biochemical and biophysical research communications, 116.

[93] Issemann, I. and Green, S. (1990) Activation of a Member of the Steroid Hormone Receptor Superfamily by Peroxisome Proliferators. Nature.

[94] Hong, S.-H., Ahmadian, M., Yu, R. T., Atkins, A. R., Downes, M., and Evans, R. M. (2014) Nuclear receptors and metabolism: from feast to famine. Diabetologia, 57, 860-7.

[95] Youssef, J. and Badr, M. Z. (2013) PPARs: History and Advances. Badr, M. Z. and Youssef, J. A. (eds.), Peroxisome Proliferator-Activated Receptors (PPARs), vol. 952 of Methods in Molecular Biology, Humana Press.

[96] Grygiel-Górniak, B. (2014) Peroxisome proliferator-activated receptors and their ligands: nutritional and clinical implications-a review. Nutrition journal, 13, 17.

[97] Juge-Aubry, C., Pernin, A., Favez, T., Burger, a. G., Wahli, W., Meier, C. a., and Desvergne, B. (1997) DNA Binding Properties of Peroxisome Proliferator- 
activated Receptor Subtypes on Various Natural Peroxisome Proliferator Response Elements: Importance of the 5'-Flanking Region. Journal of Biological Chemistry, 272, 25252-25259.

[98] Chandra, V., Huang, P., Hamuro, Y., Raghuram, S., Wang, Y., Burris, T. P., and Rastinejad, F. (2008) Structure of the intact PPAR-gamma-RXR- nuclear receptor complex on DNA. Nature, 456, 350-6.

[99] Ahmadian, M., Suh, J., Hah, N., and Liddle, C. (2013) PPARgamma signaling and metabolism: the good, the bad and the future. Nature medicine, 19.

[100] Barak, Y., Nelson, M. C., Ong, E. S., Jones, Y. Z., Ruiz-Lozano, P., Chien, K. R., Koder, a., and Evans, R. M. (1999) PPAR gamma is required for placental, cardiac, and adipose tissue development. Molecular cell, 4, 585-95.

[101] Kershaw, E. E., Schupp, M., Guan, H.-P., Gardner, N. P., Lazar, M. a., and Flier, J. S. (2007) PPARgamma regulates adipose triglyceride lipase in adipocytes in vitro and in vivo. American journal of physiology. Endocrinology and metabolism, 293, E1736-45.

[102] Imai, T., et al. (2004) Peroxisome proliferator-activated receptor gamma is required in mature white and brown adipocytes for their survival in the mouse. Proceedings of the National Academy of Sciences of the United States of America, 101, 4543-7.

[103] Masuno, H., Kidani, T., Sekiya, K., Sakayama, K., Shiosaka, T., Yamamoto, H., and Honda, K. (2002) Bisphenol A in combination with insulin can accelerate the conversion of 3T3-L1 fibroblasts to adipocytes. Journal of lipid research, 43, 676-84.

[104] Masuno, H., Iwanami, J., Kidani, T., Sakayama, K., and Honda, K. (2005) Bisphenol a accelerates terminal differentiation of 3T3-L1 cells into adipocytes through the phosphatidylinositol 3-kinase pathway. Toxicological sciences, 84, 31927.

[105] Phrakonkham, P., Viengchareun, S., Belloir, C., Lombès, M., Artur, Y., and Canivenc-Lavier, M.-C. (2008) Dietary xenoestrogens differentially impair 3T3L1 preadipocyte differentiation and persistently affect leptin synthesis. The Journal of steroid biochemistry and molecular biology, 110, 95-103.

[106] Somm, E., Schwitzgebel, V. M., Toulotte, A., Cederroth, C. R., Combescure, C., Nef, S., Aubert, M. L., and Hüppi, P. S. (2009) Perinatal exposure to bisphenol a alters early adipogenesis in the rat. Environmental health perspectives, 117, 1549-55. 
[107] Todaro, G. and Green, H. (1963) Quantitative studies of the growth of mouse embryo cells in culture and their development into established lines. The Journal of cell biology, pp. 299-313.

[108] Sato, H., Sugai, H., Kurosaki, H., Ishikawa, M., Funaki, A., Kimura, Y., and Ueno, K. (2013) The effect of sex hormones on peroxisome proliferatoractivated receptor gamma expression and activity in mature adipocytes. Biological \& pharmaceutical bulletin, 36, 564-73.

[109] Pereira-Fernandes, A., Demaegdt, H., Vandermeiren, K., Hectors, T. L. M., Jorens, P. G., Blust, R., and Vanparys, C. (2013) Evaluation of a screening system for obesogenic compounds: screening of endocrine disrupting compounds and evaluation of the PPAR dependency of the effect. PloS One, 8, e77481.

[110] Riu, A., Grimaldi, M., le Maire, A., Bey, G., Phillips, K., Boulahtouf, A., Perdu, E., Zalko, D., Bourguet, W., and Balaguer, P. (2011) Peroxisome proliferatoractivated receptor $\gamma$ is a target for halogenated analogs of bisphenol A. Environmental health perspectives, 119, 1227-32.

[111] le Maire, A., Grimaldi, M., Roecklin, D., Dagnino, S., Vivat-Hannah, V., Balaguer, P., and Bourguet, W. (2009) Activation of RXR-PPAR heterodimers by organotin environmental endocrine disruptors. EMBO reports, 10, 367-73.

[112] Nass, N. and Kalinski, T. (2015) Tamoxifen resistance: From cell culture experiments towards novel biomarkers. Pathology - Research and Practice, 211, 189-197.

[113] Klinge, C. M. (2000) Estrogen receptor interaction with co-activators and corepressors. Steroids, 65, 227-251.

[114] Li, J., Ma, M., and Wang, Z. (2008) A two-hybrid yeast assay to quantify the effects of xenobiotics on retinoid X receptor-mediated gene expression. Toxicology letters, 176, 198-206.

[115] Reynolds, R. M. (2013) Glucocorticoid excess and the developmental origins of disease: Two decades of testing the hypothesis - 2012 Curt Richter Award Winner. Psychoneuroendocrinology, 38, 1-11.

[116] Atlas, E., Pope, L., Wade, M. G., Kawata, A., Boudreau, A., and Boucher, J. G. (2014) Bisphenol A increases aP2 expression in 3T3L1 by enhancing the transcriptional activity of nuclear receptors at the promoter. Adipocyte, 3, 170-9.

[117] Wang, J., Sun, B., Hou, M., Pan, X., and Li, X. (2013) The environmental obesogen bisphenol A promotes adipogenesis by increasing the amount of $11 \beta$ - 
hydroxysteroid dehydrogenase type 1 in the adipose tissue of children. International journal of obesity, 37, 999-1005.

[118] Prasanth, G. K., Divya, L. M., and Sadasivan, C. (2010) Bisphenol-A can bind to human glucocorticoid receptor as an agonist: An in silico study. Journal of Applied Toxicology, 30, 769-774.

[119] Sargis, R. M., Johnson, D. N., Choudhury, R. a., and Brady, M. J. (2010) Environmental endocrine disruptors promote adipogenesis in the 3T3-L1 cell line through glucocorticoid receptor activation. Obesity, 18, 1283-8.

[120] Gorski, R. a., Gordon, J. H., Shryne, J. E., and Southam, a. M. (1978) Evidence for a morphological sex difference within the medial preoptic area of the rat brain. Brain Research, 148, 333-346.

[121] Arai, Y., Sekine, Y., and Murakami, S. (1996) Estrogen and apoptosis in the developing sexuallyimorpic preoptic area in female rats. Neuroscience Research, 25, 403-407.

[122] Nagao, T., Saito, Y., Usumi, K., Kuwagata, M., and Imai, K. (1999) Reproductive function in rats exposed neonatally to bisphenol A and estradiol benzoate. Reproductive toxicology, 13, 303-311.

[123] Kwon, S., Stedman, D. B., Elswick, B. a., Cattley, R. C., and Welsch, F. (2000) Pubertal development and reproductive functions of Crl:CD BR Sprague-Dawley rats exposed to bisphenol A during prenatal and postnatal development. Toxicological sciences, 55, 399-406.

[124] He, Z., Paule, M. G., and Ferguson, S. a. (2012) Low oral doses of bisphenol A increase volume of the sexually dimorphic nucleus of the preoptic area in male, but not female, rats at postnatal day 21. Neurotoxicology and Teratology, 34, 331-337.

[125] Patisaul, H. B., Fortino, A. E., and Polston, E. K. (2007) Differential disruption of nuclear volume and neuronal phenotype in the preoptic area by neonatal exposure to genistein and bisphenol-A. NeuroToxicology, 28, 1-12.

[126] McCaffrey, K. a., Jones, B., Mabrey, N., Weiss, B., Swan, S. H., and Patisaul, H. B. (2013) Sex specific impact of perinatal bisphenol A (BPA) exposure over a range of orally administered doses on rat hypothalamic sexual differentiation. NeuroToxicology, 36, 55-62.

[127] Sickel, M. J. and McCarthy, M. M. (2000) Calbindin-d28k immunoreactivity is a marker for a subdivision of the sexually dimorphic nucleus of the preoptic 
area of the rat: Developmental profile and gonadal steroid modulation. Journal of Neuroendocrinology, 12, 397-402.

[128] He, Z., Ferguson, S. A., Cui, L., Greenfield, L. J., and Paule, M. G. (2013) Development of the sexually dimorphic nucleus of the preoptic area and the influence of estrogen-like compounds. Neural Regeneration Research, 8, 2763-2774.

[129] Waters, E. M. and Simerly, R. B. (2009) Estrogen induces caspase-dependent cell death during hypothalamic development. The Journal of Neuroscience, 29, 9714-8.

[130] Rubin, B. S., Lenkowski, J. R., Schaeberle, C. M., Vandenberg, L. N., Ronsheim, P. M., and Soto, A. M. (2006) Evidence of altered brain sexual differentiation in mice exposed perinatally to low, environmentally relevant levels of bisphenol A. Endocrinology, 147, 3681-3691.

[131] Bai, Y., Chang, F., Zhou, R., Jin, P. P., Matsumoto, H., Sokabe, M., and Chen, L. (2011) Increase of anteroventral periventricular kisspeptin neurons and generation of E2-induced LH-surge system in male rats exposed perinatally to environmental dose of bisphenol-A. Endocrinology, 152, 1562-1571.

[132] Patisaul, H. B., Todd, K. L., Mickens, J. a., and Adewale, H. B. (2009) Impact of neonatal exposure to the ERalpha agonist PPT, bisphenol-A or phytoestrogens on hypothalamic kisspeptin fiber density in male and female rats. Neurotoxicology, 30, 350-7.

[133] Adewale, H. B., Todd, K. L., Mickens, J. a., and Patisaul, H. B. (2011) The impact of neonatal bisphenol-A exposure on sexually dimorphic hypothalamic nuclei in the female rat. NeuroToxicology, 32, 38-49.

[134] Nagel, S. and vom Saal, F. (1997) Relative binding affinity-serum modified access (RBA-SMA) assay predicts the relative in vivo bioactivity of the xenoestrogens bisphenol A and octylphenol. Environmental Health Perspectives, 105.

[135] Howdeshell, K., Hotchkiss, A., Thayer, K., Vandenbergh, J., and vom Saal, F. (1999) Environmental toxins: exposure to bisphenol A advances puberty. $\mathrm{Na}$ ture, pp. 763-764.

[136] Rubin, B. S., Murray, M. K., Damassa, D. a., King, J. C., and Soto, a. M. (2001) Perinatal exposure to low doses of bisphenol A affects body weight, patterns of estrous cyclicity, and plasma LH levels. Environmental health perspectives, 109, 675-80. 
[137] Nikaido, Y., Yoshizawa, K., Danbara, N., Tsujita-Kyutoku, M., Yuri, T., Uehara, N., and Tsubura, A. (2004) Effects of maternal xenoestrogen exposure on development of the reproductive tract and mammary gland in female CD-1 mouse offspring. Reproductive toxicology, 18, 803-11.

[138] Takai, Y., Tsutsumi, O., Ikezuki, Y., Kamei, Y., Osuga, Y., Yano, T., and Taketan, Y. (2001) Preimplantation exposure to bisphenol A advances postnatal development. Reproductive toxicology, 15, 71-4.

[139] Miyawaki, J., Sakayama, K., Kato, H., Yamamoto, H., and Masuno, H. (2007) Perinatal and postnatal exposure to bisphenol a increases adipose tissue mass and serum cholesterol level in mice. Journal of atherosclerosis and thrombosis, 14, 245-52.

[140] Alonso-Magdalena, P., Vieira, E., Soriano, S., Menes, L., Burks, D., Quesada, I., and Nadal, A. (2010) Bisphenol A exposure during pregnancy disrupts glucose homeostasis in mothers and adult male offspring. Environmental health perspectives, $118,1243-50$.

[141] Angle, B. M., et al. (2013) Metabolic disruption in male mice due to fetal exposure to low but not high doses of bisphenol A (BPA): evidence for effects on body weight, food intake, adipocytes, leptin, adiponectin, insulin and glucose regulation. Reproductive toxicology, 42, 256-68.

[142] García-Arevalo, M., Alonso-Magdalena, P., Rebelo Dos Santos, J., Quesada, I., Carneiro, E. M., and Nadal, A. (2014) Exposure to Bisphenol-A during Pregnancy Partially Mimics the Effects of a High-Fat Diet Altering Glucose Homeostasis and Gene Expression in Adult Male Mice. PloS one, 9, e100214.

[143] Ryan, K. K., Haller, A. M., Sorrell, J. E., Woods, S. C., Jandacek, R. J., and Seeley, R. J. (2010) Perinatal exposure to bisphenol-a and the development of metabolic syndrome in CD-1 mice. Endocrinology, 151, 2603-12.

[144] Wei, J., et al. (2011) Perinatal exposure to bisphenol A at reference dose predisposes offspring to metabolic syndrome in adult rats on a high-fat diet. Endocrinology, 152, 3049-61.

[145] Tyl, R. W., et al. (2008) Two-generation reproductive toxicity study of dietary bisphenol A in CD-1 (Swiss) mice. Toxicological sciences, 104, 362-84.

[146] Tyl, R. W., et al. (2002) Three-generation reproductive toxicity study of dietary bisphenol A in CD Sprague-Dawley rats. Toxicological sciences, 68, 121-46. 
[147] Wei, J., Sun, X., Chen, Y., Li, Y., Song, L., Zhou, Z., Xu, B., Lin, Y., and $\mathrm{Xu}, \mathrm{S}$. (2014) Perinatal exposure to bisphenol A exacerbates nonalcoholic steatohepatitis-like phenotype in male rat offspring fed on a high-fat diet. The Journal of endocrinology, 222, 313-25.

[148] National Toxicology Program (2008) NTP-CERHR monograph on the potential human reproductive and developmental effects of bisphenol A. National Institutes of Health.

[149] Brown, a. P., Dinger, N., and Levine, B. S. (2000) Stress produced by gavage administration in the rat. Contemporary topics in laboratory animal science, 39, 17-21.

[150] vom Saal, F. S. and Welshons, W. V. (2006) Large effects from small exposures. II. The importance of positive controls in low-dose research on bisphenol A. Environmental research, 100, 50-76.

[151] Dodds, E. C., Goldberg, L., Lawson, W., and Robinson, R. (1938), Estrogenic Activity of Certain Synthetic Compounds.

[152] Korach, K. S., Metzler, M., and McLachlan, J. a. (1978) Estrogenic activity in vivo and in vitro of some diethylstilbestrol metabolites and analogs. Proceedings of the National Academy of Sciences of the United States of America, 75, 468-471.

[153] Folmar, L. C., Hemmer, M. J., Denslow, N. D., Kroll, K., Chen, J., Cheek, A., Richman, H., Meredith, H., and Grau, E. G. (2002) A comparison of the estrogenic potencies of estradiol, ethynylestradiol, diethylstilbestrol, nonylphenol and methoxychlor in vivo and in vitro. Aquatic Toxicology, 60, 101-110.

[154] Faber, K. a., Ayyash, L., Dixon, S., and Hughes, C. L. (1993) Effect of neonatal diethylstilbestrol exposure on volume of the sexually dimorphic nucleus of the preoptic area of the hypothalamus and pituitary responsiveness to gonadotropinreleasing hormone in female rats of known anogenital distance at birth. Biology of reproduction, 48, 947-51.

[155] Döhler, K. D., Coquelin, a., Davis, F., Hines, M., Shryne, J. E., and Gorski, R. a. (1984) Pre- and postnatal influence of testosterone propionate and diethylstilbestrol on differentiation of the sexually dimorphic nucleus of the preoptic area in male and female rats. Brain research, 302, 291-5.

[156] Yamamoto, M., Shirai, M., Tamura, A., Kobayashi, T., Kohara, S., Murakami, M., and Arishima, K. (2005) Effects of maternal exposure to a low dose of diethylstilbestrol on sexual dimorphic nucleus volume and male reproductive system in rat offspring. The Journal of toxicological sciences, 30, 7-18. 
[157] Newbold, R. R., Padilla-Banks, E., Jefferson, W. N., and Heindel, J. J. (2008) Effects of endocrine disruptors on obesity. International journal of andrology, 31, 201-8.

[158] Newbold, R. R., Padilla-Banks, E., Snyder, R. J., and Jefferson, W. N. (2005) Developmental exposure to estrogenic compounds and obesity. Birth defects research. Part A, Clinical and molecular teratology, 73, 478-80.

[159] Hao, C.-J., Cheng, X.-J., Xia, H.-F., and Ma, X. (2012) The endocrine disruptor diethylstilbestrol induces adipocyte differentiation and promotes obesity in mice. Toxicology and applied pharmacology, 263, 102-10.

[160] Thigpen, J. E., et al. (2013) The estrogenic content of rodent diets, bedding, cages, and water bottles and its effect on bisphenol a studies. Journal of the American Association for Laboratory Animal Science, 52, 130-41.

[161] Haseman, J. K., Bailer, a. J., Kodell, R. L., Morris, R., and Portier, K. (2001) Statistical issues in the analysis of low-dose endocrine disruptor data. Toxicological sciences : an official journal of the Society of Toxicology, 61, 201-10.

[162] Lazic, S. E. (2010) The problem of pseudoreplication in neuroscientific studies: is it affecting your analysis? BMC neuroscience, 11, 5 .

[163] Holson, R. R. and Pearce, B. (1992) Principles and pitfalls in the analysis of prenatal treatment effects in multiparous species. Neurotoxicology and teratology, 14, $221-8$.

[164] Lazic, S. E. and Essioux, L. (2013) Improving basic and translational science by accounting for litter-to-litter variation in animal models. BMC neuroscience, 14, 37.

[165] Hetherington, A. and Ranson, S. (1940) Hypothalamic lesions and adiposity in the rat. The Anatomical Record.

[166] Rossi, M., et al. (1998) A C-terminal fragment of Agouti-related protein increases feeding and antagonizes the effect of alpha-melanocyte stimulating hormone in vivo. Endocrinology, 139, 4428-4431.

[167] Billington, C. J. and Levine, A. S. (1992) Hypothalamic neuropeptide Y regulation of feeding and energy metabolism. Current opinion in neurobiology, 2, 847-851.

[168] Fan, W., Boston, B., Kesterson, R., Hruby, V. J., and Cone, R. D. (1997) Role of melanocortinergic neurons in feeding and the agouti obesity syndrome. Nature, 385, 165-168. 
[169] Zhang, Y., Proenca, R., Maffei, M., Barone, M., Leopold, L., and Friedman, J. (1994) Positional cloning of the mouse obese gene and its human homologue. Nature.

[170] Maffei, M., Halaas, J., Ravussin, E., Pratley, R. E., Lee, G. H., Zhang, Y., Fei, H., Kim, S., Lallone, R., and Ranganathan, S. (1995) Leptin levels in human and rodent: measurement of plasma leptin and ob RNA in obese and weight-reduced subjects. Nature medicine, 1, 1155-1161.

[171] De Luca, C., Kowalski, T. J., Zhang, Y., Elmquist, J. K., Lee, C., Kilimann, M. W., Ludwig, T., Liu, S. M., and Chua, S. C. (2005) Complete rescue of obesity, diabetes, and infertility in $\mathrm{db} / \mathrm{db}$ mice by neuron-specific LEPR-B transgenes. Journal of Clinical Investigation, 115, 3484-3493.

[172] Bjorbak, C., Lavery, H. J., Bates, S. H., Olson, R. K., Davis, S. M., Flier, J. S., and Myers, M. G. (2000) SOCS3 mediates feedback inhibition of the leptin receptor via Tyr985. The Journal of biological chemistry, 275, 40649-57.

[173] Banks, A., Davis, S., Bates, S., and Myers, M. (2000) Activation of downstream signals by the long form of the leptin receptor. Journal of Biological Chemistry, 275, 14563-14572.

[174] Bjorbaek, C., Uotani, S., da Silva, B., and Flier, J. S. (1997) Divergent signaling capacities of the long and short isoforms of the leptin receptor. The Journal of biological chemistry, 272, 32686-95.

[175] Xu, A. W., Ste-Marie, L., Kaelin, C. B., and Barsh, G. S. (2007) Inactivation of signal transducer and activator of transcription 3 in proopiomelanocortin (Pomc) neurons causes decreased pomc expression, mild obesity, and defects in compensatory refeeding. Endocrinology, 148, 72-80.

[176] Munzberg, H., Huo, L., Nillni, E. a., Hollenberg, A. N., and Bjorbaek, C. (2003) Role of Signal Transducer and Activator of Transcription 3 in Regulation of Hypothalamic Proopiomelanocortin Gene Expression by Leptin. Endocrinology, 144, 2121-2131.

[177] Enriori, P. J., et al. (2007) Diet-induced obesity causes severe but reversible leptin resistance in arcuate melanocortin neurons. Cell metabolism, 5, 181-94.

[178] Münzberg, H., Flier, J. S., and Bjø rbaek, C. (2004) Region-specific leptin resistance within the hypothalamus of diet-induced obese mice. Endocrinology, 145, 4880-9. 
[179] Elias, C. F., Aschkenasi, C., Lee, C., Kelly, J., Ahima, R. S., Bjorbæ k, C., Flier, J. S., Saper, C. B., and Elmquist, J. K. (1999) Leptin differentially regulates NPY and POMC neurons projecting to the lateral hypothalamic area. Neuron, 23, 775-786.

[180] Cowley, M. a., Smart, J. L., Rubinstein, M., Cerdán, M. G., Diano, S., Horvath, T. L., Cone, R. D., and Low, M. J. (2001) Leptin activates anorexigenic POMC neurons through a neural network in the arcuate nucleus. Nature, 411, 480-484.

[181] Pinto, S., Roseberry, A. G., Liu, H., Diano, S., Shanabrough, M., Cai, X., Friedman, J. M., and Horvath, T. L. (2004) Rapid rewiring of arcuate nucleus feeding circuits by leptin. Science, 304, 110-5.

[182] Gao, Q., et al. (2007) Anorectic estrogen mimics leptin's effect on the rewiring of melanocortin cells and Stat3 signaling in obese animals. Nature medicine, 13, 89-94.

[183] Dietrich, M. O. and Horvath, T. L. (2013) Hypothalamic control of energy balance: insights into the role of synaptic plasticity. Trends in neurosciences, 36, 65-73.

[184] Seeley, R. J., Yagaloff, K. a., Fisher, S. L., Burn, P., Thiele, T. E., van Dijk, G., Baskin, D. G., and Schwartz, M. W. (1997) Melanocortin receptors in leptin effects. Nature, 390, 349.

[185] Bouret, S. G. and Simerly, R. B. (2007) Development of leptin-sensitive circuits. Journal of neuroendocrinology, 19, 575-82.

[186] Lenard, N. and Berthoud, H. (2008) Central and Peripheral Regulation of Food Intake and Physical Activity: Pathways and Genes. Obesity, 16.

[187] Ollmann, M. M., Wilson, B. D., Yang, Y. K., Kerns, J. a., Chen, Y., Gantz, I., and Barsh, G. S. (1997) Antagonism of central melanocortin receptors in vitro and in vivo by agouti-related protein. Science, 278, 135-138.

[188] Vickers, M. and Breier, B. (2000) Fetal origins of hyperphagia , obesity , and hypertension and postnatal amplification by hypercaloric nutrition. American Journal of Physiology, Endocrinology, and Metabolism, 279.

[189] Desai, M., Gayle, D., Han, G., and Ross, M. G. (2007) Programmed hyperphagia due to reduced anorexigenic mechanisms in intrauterine growth-restricted offspring. Reproductive sciences, 14, 329-37.

[190] Manuel-Apolinar, L., Zarate, A., Rocha, L., and Hernández, M. (2010) Fetal malnutrition affects hypothalamic leptin receptor expression after birth in male mice. Archives of medical research, 41, 240-5. 
[191] Garcia, A., Palou, M., and Priego, T. (2010) Moderate caloric restriction during gestation results in lower arcuate nucleus NPY- and $\alpha \mathrm{MSH}$-neurons and impairs hypothalamic response to fed/fasting conditions in weaned rats. Diabetes, Obesity and Metabolism, pp. 403-413.

[192] Ikenasio-Thorpe, B. a., Breier, B. H., Vickers, M. H., and Fraser, M. (2007) Prenatal influences on susceptibility to diet-induced obesity are mediated by altered neuroendocrine gene expression. The Journal of endocrinology, 193, 31-7.

[193] Shin, B.-C., Dai, Y., Thamotharan, M., Gibson, L. C., and Devaskar, S. U. (2012) Pre- and postnatal calorie restriction perturbs early hypothalamic neuropeptide and energy balance. Journal of neuroscience research, 90, 1169-82.

[194] Terroni, P. L., Anthony, F. W., Hanson, M. a., and Cagampang, F. R. a. (2005) Expression of agouti-related peptide, neuropeptide Y, pro-opiomelanocortin and the leptin receptor isoforms in fetal mouse brain from pregnant dams on a protein-restricted diet. Molecular brain research, 140, 111-5.

[195] Krechowec, S. O., Vickers, M., Gertler, A., and Breier, B. H. (2006) Prenatal influences on leptin sensitivity and susceptibility to diet-induced obesity. The Journal of endocrinology, 189, 355-63.

[196] Chen, H., Simar, D., and Morris, M. J. (2009) Hypothalamic neuroendocrine circuitry is programmed by maternal obesity: interaction with postnatal nutritional environment. PloS one, 4, e6259.

[197] Kirk, S. L., Samuelsson, A.-M., Argenton, M., Dhonye, H., Kalamatianos, T., Poston, L., Taylor, P. D., and Coen, C. W. (2009) Maternal obesity induced by diet in rats permanently influences central processes regulating food intake in offspring. PloS one, 4, e5870.

[198] Rajia, S., Chen, H., and Morris, M. J. (2010) Maternal overnutrition impacts offspring adiposity and brain appetite markers-modulation by postweaning diet. Journal of neuroendocrinology, 22, 905-14.

[199] Morris, M. J. and Chen, H. (2009) Established maternal obesity in the rat reprograms hypothalamic appetite regulators and leptin signaling at birth. International journal of obesity, 33, 115-22.

[200] Glavas, M. M., Kirigiti, M. a., Xiao, X. Q., Enriori, P. J., Fisher, S. K., Evans, A. E., Grayson, B. E., Cowley, M. a., Smith, M. S., and Grove, K. L. (2010) Early overnutrition results in early-onset arcuate leptin resistance and increased sensitivity to high-fat diet. Endocrinology, 151, 1598-610. 
[201] Chen, H., Simar, D., Lambert, K., Mercier, J., and Morris, M. J. (2008) Maternal and postnatal overnutrition differentially impact appetite regulators and fuel metabolism. Endocrinology, 149, 5348-56.

[202] Bouret, S. G., Gorski, J. N., Patterson, C. M., Chen, S., Levin, B. E., and Simerly, R. B. (2008) Hypothalamic neural projections are permanently disrupted in dietinduced obese rats. Cell metabolism, 7, 179-85.

[203] Simerly, R. B. (2008) Hypothalamic substrates of metabolic imprinting. Physiology \& behavior, 94, 79-89.

[204] Gupta, A., Srinivasan, M., Thamadilok, S., and Patel, M. S. (2009) Hypothalamic alterations in fetuses of high fat diet-fed obese female rats. The Journal of endocrinology, 200, 293-300.

[205] Ahima, R. S., Prabakaran, D., and Flier, J. S. (1998) Postnatal leptin surge and regulation of circadian rhythm of leptin by feeding. Implications for energy homeostasis and neuroendocrine function. The Journal of clinical investigation, 101, $1020-7$.

[206] Delahaye, F., Breton, C., Risold, P.-Y., Enache, M., Dutriez-Casteloot, I., Laborie, C., Lesage, J., and Vieau, D. (2008) Maternal perinatal undernutrition drastically reduces postnatal leptin surge and affects the development of arcuate nucleus proopiomelanocortin neurons in neonatal male rat pups. Endocrinology, 149, 4705.

[207] Yura, S., et al. (2005) Role of premature leptin surge in obesity resulting from intrauterine undernutrition. Cell metabolism, 1, 371-8.

[208] Brobeck, J. (1947) Variations in regulation of energy exchange associated with estrus, diestrus and pseudopregnancy in rats. Endocrinology, 40, 65-72.

[209] Blaustein, J. D. and Wade, G. N. (1976) Ovarian influences on the meal patterns of female rats. Physiology \& behavior, 17, 201-8.

[210] Drewett, R. (1973) Oestrous and dioestrous components of the ovarian inhibition on hunger in the rat. Animal behaviour, pp. 772-780.

[211] Drewett, R. F. (1974) The meal patterns of the oestrous cycle and their motivational significance. The Quarterly journal of experimental psychology, 26, 489-94.

[212] Asarian, L. and Geary, N. (2006) Modulation of appetite by gonadal steroid hormones. Philosophical transactions of the Royal Society of London. Series B, Biological sciences, 361, 1251-63. 
[213] Eckel, L. a. (2011) The ovarian hormone estradiol plays a crucial role in the control of food intake in females. Physiology \& behavior, 104, 517-24.

[214] Chai, J. and Blaha, V. (1999) Use of orchiectomy and testosterone replacement to explore meal number-to-meal size relationship in male rats. American Journal of Physiology, pp. 1366-1373.

[215] Wallen, W. J., Belanger, M. P., and Wittnich, C. (2001) Sex hormones and the selective estrogen receptor modulator tamoxifen modulate weekly body weights and food intakes in adolescent and adult rats. The Journal of nutrition, 131, 2351-7.

[216] McElroy, J. F. and Wade, G. N. (1987) Short- and long-term effects of ovariectomy on food intake, body weight, carcass composition, and brown adipose tissue in rats. Physiology \& behavior, 39, 361-5.

[217] Wade, G. N. (1975) Some effects of ovarian hormones on food intake and body weight in female rats. Journal of Comparative and Physiological Psychology, 88, 183-193.

[218] Varma, M., Chai, J. K., Meguid, M. M., Laviano, a., Gleason, J. R., Yang, Z. J., and Blaha, V. (1999) Effect of estradiol and progesterone on daily rhythm in food intake and feeding patterns in Fischer rats. Physiology \& behavior, 68, 99-107.

[219] Asarian, L. and Geary, N. (1999) Cyclic estradiol treatment phasically potentiates endogenous cholecystokinin's satiating action in ovariectomized rats. Peptides, 20, 445-450.

[220] Asarian, L. and Geary, N. (2002) Cyclic Estradiol Treatment Normalizes Body Weight and Restores Physiological Patterns of Spontaneous Feeding and Sexual Receptivity in Ovariectomized Rats. Hormones and Behavior, 42, 461-471.

[221] Gallo, M. and Grimes, D. (2014) Combination contraceptives: effects on weight. Cochrane Database of Systematic Reviews.

[222] Rogers, N. H., Perfield, J. W., Strissel, K. J., Obin, M. S., and Greenberg, A. S. (2009) Reduced energy expenditure and increased inflammation are early events in the development of ovariectomy-induced obesity. Endocrinology, 150, 2161-8.

[223] Laudenslager, M., Wilkinson, C., Carlisle, H., and Hammel, H. (1980) Energy balance in ovariectomized with and without estrogen replacement. American Journal of Physiology, 238.

[224] Parker, G. C., McKee, M. E., Bishop, C., and Coscina, D. V. (2001) Whole-body metabolism varies across the estrous cycle in Sprague-Dawley rats. Physiology \& Behavior, 74, 399-403. 
[225] Giles, E. and Jackman, M. (2010) Effect of the estrous cycle and surgical ovariectomy on energy balance, fuel utilization, and physical activity in lean and obese female rats. American Journal of Physiology - Regulatory, Integrative and Comparative Physiology, 80045, 1634-1642.

[226] Martínez de Morentin, P. B., et al. (2014) Estradiol Regulates Brown Adipose Tissue Thermogenesis via Hypothalamic AMPK. Cell metabolism, 20, 41-53.

[227] Litwak, S. a., Wilson, J. L., Chen, W., Garcia-Rudaz, C., Khaksari, M., Cowley, M. a., and Enriori, P. J. (2014) Estradiol prevents fat accumulation and overcomes leptin resistance in female high fat diet mice. Endocrinology, p. en20141342.

[228] Heine, P. a., Taylor, J. a., Iwamoto, G. a., Lubahn, D. B., and Cooke, P. S. (2000) Increased adipose tissue in male and female estrogen receptor-alpha knockout mice. Proceedings of the National Academy of Sciences of the United States of America, 97, 12729-34.

[229] Geary, N., Asarian, L., Korach, K., Pfaff, D., and Ogawa, S. (2001) Deficits in E2-Dependent Control of Feeding, Weight Gain, and Cholecystokinin Satiation in ER- $\alpha$ Null Mice. Endocrinology, 142, 4751-4757.

[230] Jones, M. E., et al. (2000) Aromatase-deficient (ArKO) mice have a phenotype of increased adiposity. Proceedings of the National Academy of Sciences of the United States of America, 97, 12735-40.

[231] Jones, M., Thorburn, A., and Britt, K. (2001) Aromatase-deficient ( ArKO ) mice accumulate excess adipose tissue. The Journal of steroid biochemistry and molecular biology, 79, 3-9.

[232] Rivera, H. M. and Eckel, L. a. (2010) Activation of central, but not peripheral, estrogen receptors is necessary for estradiol's anorexigenic effect in ovariectomized rats. Endocrinology, 151, 5680-8.

[233] Musatov, S., Chen, W., Pfaff, D. W., Mobbs, C. V., Yang, X.-J., Clegg, D. J., Kaplitt, M. G., and Ogawa, S. (2007) Silencing of estrogen receptor alpha in the ventromedial nucleus of hypothalamus leads to metabolic syndrome. Proceedings of the National Academy of Sciences of the United States of America, 104, 2501-6.

[234] Xu, Y., et al. (2011) Distinct hypothalamic neurons mediate estrogenic effects on energy homeostasis and reproduction. Cell metabolism, 14, 453-65.

[235] Shi, H., Sorrell, J. E., Clegg, D. J., Woods, S. C., and Seeley, R. J. (2010) The roles of leptin receptors on POMC neurons in the regulation of sex-specific energy homeostasis. Physiology \& behavior, 100, 165-72. 
[236] Fodor, M. and Delemarre-van de Waal, H. a. (2001) Are POMC neurons targets for sex steroids in the arcuate nucleus of the rat? Neuroreport, 12, 3989-91.

[237] de Souza, F. S. J., Nasif, S., López-Leal, R., Levi, D. H., Low, M. J., and Rubinsten, M. (2011) The estrogen receptor $\alpha$ colocalizes with proopiomelanocortin in hypothalamic neurons and binds to a conserved motif present in the neuronspecific enhancer nPE2. European journal of pharmacology, 660, 181-7.

[238] Nohara, K., Zhang, Y., Waraich, R. S., Laque, A., Tiano, J. P., Tong, J., Münzberg, H., and Mauvais-Jarvis, F. (2011) Early-life exposure to testosterone programs the hypothalamic melanocortin system. Endocrinology, 152, 1661-9.

[239] Pelletier, G., Li, S., Luu-The, V., and Labrie, F. (2007) Oestrogenic regulation of pro-opiomelanocortin, neuropeptide $\mathrm{Y}$ and corticotrophin-releasing hormone mRNAs in mouse hypothalamus. Journal of neuroendocrinology, 19, 426-31.

[240] Medina, F., Siddiqui, A., Scimonelli, T., Fenske, C., Wilson, C. A., and Celis, M. E. (1998) The inter-relationship between gonadal steroids and POMC peptides, beta-endorphin and alpha-MSH, in the control of sexual behavior in the female rat. Peptides, 19, 1309-1316.

[241] Olofsson, L. E., Pierce, A. a., and Xu, A. W. (2009) Functional requirement of AgRP and NPY neurons in ovarian cycle-dependent regulation of food intake. Proceedings of the National Academy of Sciences of the United States of America, 106, 15932-7.

[242] Kwon, O., Kang, E. S., Kim, I., Shin, S., Kim, M., Kwon, S., Oh, S. R., Ahn, Y. S., and Kim, C. H. (2014) GPR30 mediates anorectic estrogen-induced STAT3 signaling in the hypothalamus. Metabolism: clinical and experimental, 63, 1455-61.

[243] Bennett, P. a., Lindell, K., Wilson, C., Carlsson, L. M., Carlsson, B., and Robinson, I. C. (1999) Cyclical variations in the abundance of leptin receptors, but not in circulating leptin, correlate with NPY expression during the oestrous cycle. Neuroendocrinology, 69, 417-23.

[244] Fungfuang, W., Nakada, T., Nakao, N., Terada, M., Yokosuka, M., Gizurarson, S., Hau, J., Moon, C., and Saito, T. R. (2013) Serum leptin concentrations, leptin mRNA expression, and food intake during the estrous cycle in rats. Laboratory animal research, 29, 1-6.

[245] da Silva, R. P., Zampieri, T. T., Pedroso, J. a. a. B., Nagaishi, V. S., Ramos-Lobo, A. M., Furigo, I. C., Câmara, N. O., Frazão, R., and Donato, J. (2014) Leptin resistance is not the primary cause of weight gain associated with reduced sex hormone levels in female mice. Endocrinology, 155, 4226-36. 
[246] Hong, J., Stubbins, R. E., Smith, R. R., Harvey, A. E., and Núñez, N. P. (2009) Differential susceptibility to obesity between male, female and ovariectomized female mice. Nutrition journal, 8, 11.

[247] Funabashi, T., Hagiwara, H., Mogi, K., Mitsushima, D., Shinohara, K., and Kimura, F. (2009) Sex differences in the responses of orexin neurons in the lateral hypothalamic area and feeding behavior to fasting. Neuroscience letters, 463, $31-4$.

[248] Swanson, H. and Bosch, J. T. (1963) Sex Differences in the Growth of Rats, and Their Modification by a Single Injection of Testosterone Proprionate Shortly After Birth. Journal of Endocrinology.

[249] Beatty, W. W., Powley, T. L., and Keesey, R. E. (1970) Effects of neonatal testosterone injection and hormone replacement in adulthood on body weight and body fat in female rats. Physiology \& behavior, 5, 1093-8.

[250] Nohara, K., Liu, S., Meyers, M. S., Waget, A., Ferron, M., Karsenty, G., Burcelin, R., and Mauvais-Jarvis, F. (2013) Developmental androgen excess disrupts reproduction and energy homeostasis in adult male mice. The Journal of endocrinology, 219, 259-68.

[251] Plagemann, A., et al. (2009) Hypothalamic proopiomelanocortin promoter methylation becomes altered by early overfeeding: an epigenetic model of obesity and the metabolic syndrome. The Journal of physiology, 587, 4963-76.

[252] Marco, A., Kisliouk, T., Weller, A., and Meiri, N. (2013) High fat diet induces hypermethylation of the hypothalamic Pomc promoter and obesity in post-weaning rats. Psychoneuroendocrinology.

[253] Coupé, B., Amarger, V., Grit, I., Benani, A., and Parnet, P. (2010) Nutritional programming affects hypothalamic organization and early response to leptin. Endocrinology, 151, 702-13.

[254] Palou, M., Picó, C., McKay, J. a., Sánchez, J., Priego, T., Mathers, J. C., and Palou, a. (2011) Protective effects of leptin during the suckling period against later obesity may be associated with changes in promoter methylation of the hypothalamic pro-opiomelanocortin gene. The British journal of nutrition, 106, 76978.

[255] Wang, X., Lacza, Z., Sun, Y. E., and Han, W. (2013) Leptin resistance and obesity in mice with deletion of methyl-CpG-binding protein 2 (MeCP2) in hypothalamic pro-opiomelanocortin (POMC) neurons. Diabetologia, 2. 
[256] Yang, G., Lim, C.-Y., Li, C., Xiao, X., Radda, G. K., Li, C., Cao, X., and Han, W. (2009) FoxO1 inhibits leptin regulation of pro-opiomelanocortin promoter activity by blocking STAT3 interaction with specificity protein 1 . The Journal of biological chemistry, 284, 3719-27.

[257] Jeannotte, L. and Trifiro, M. (1987) Tissue-Specific Activity of the ProOpiomelanocortin Gene Promoter. Molecular and cellular biology, 7.

[258] Pelletier, G. (1993) Regulation of proopiomelanocortin gene expression in rat brain and pituitary as studied by in situ hybridization. Annals of the New York Academy of Sciences, 680, 246-59.

[259] de Souza, F., Santangelo, A., Bumaschny, V., Avale, M., Smart, J., Low, M., and Rubinstein, M. (2005) Identification of neuronal enhancers of the proopiomelanocortin gene by transgenic mouse analysis and phylogenetic footprinting. Molecular and Cellular Biology.

[260] McCarthy, M. M., Auger, A. P., Bale, T. L., De Vries, G. J., Dunn, G. a., Forger, N. G., Murray, E. K., Nugent, B. M., Schwarz, J. M., and Wilson, M. E. (2009) The epigenetics of sex differences in the brain. The Journal of neuroscience, 29, 12815-23.

[261] Kurian, J. R., Olesen, K. M., and Auger, A. P. (2010) Sex differences in epigenetic regulation of the estrogen receptor-alpha promoter within the developing preoptic area. Endocrinology, 151, 2297-305.

[262] Schwarz, J. M., Nugent, B. M., and McCarthy, M. M. (2010) Developmental and hormone-induced epigenetic changes to estrogen and progesterone receptor genes in brain are dynamic across the life span. Endocrinology, 151, 4871-81.

[263] Kundakovic, M. and Champagne, F. a. (2011) Epigenetic perspective on the developmental effects of bisphenol A. Brain, behavior, and immunity, 25, 1084-93.

[264] Dolinoy, D. (2008) The agouti mouse model: an epigenetic biosensor for nutritional and environmental alterations on the fetal epigenome. Nutrition reviews, 66, 1-8.

[265] Dolinoy, D. C., Huang, D., and Jirtle, R. L. (2007) Maternal nutrient supplementation counteracts bisphenol A-induced DNA hypomethylation in early development. Proceedings of the National Academy of Sciences of the United States of America, 104, 13056-61. 
[266] Anderson, O., Nahar, M., and Faulk, C. (2012) Epigenetic Responses Following Maternal Dietary Exposure to Physiologically Relevant Levels of Bisphenol A. Environmental and Molecular Mutagenesis.

[267] Rosenfeld, C. S., Sieli, P. T., Warzak, D. a., Ellersieck, M. R., Pennington, K. a., and Roberts, R. M. (2013) Maternal exposure to bisphenol A and genistein has minimal effect on Avy/a offspring coat color but favors birth of agouti over nonagouti mice. Proceedings of the National Academy of Sciences of the United States of America, 110, 537-42.

[268] Warita, K., Mitsuhashi, T., Ohta, K.-I., Suzuki, S., Hoshi, N., Miki, T., and Takeuchi, Y. (2013) Gene expression of epigenetic regulatory factors related to primary silencing mechanism is less susceptible to lower doses of bisphenol A in embryonic hypothalamic cells. The Journal of toxicological sciences, 38, 285-9.

[269] Kundakovic, M., Gudsnuk, K., Franks, B., Madrid, J., Miller, R. L., Perera, F. P., and Champagne, F. a. (2013) Sex-specific epigenetic disruption and behavioral changes following low-dose in utero bisphenol A exposure. Proceedings of the $\mathrm{Na}$ tional Academy of Sciences, pp. 1-6.

[270] Richter, C. a., Birnbaum, L. S., Farabollini, F., Newbold, R. R., Rubin, B. S., Talsness, C. E., Vandenbergh, J. G., Walser-Kuntz, D. R., and vom Saal, F. S. (2007) In vivo effects of bisphenol A in laboratory rodent studies. Reproductive toxicology, 24, 199-224.

[271] Rubin, B. S. (2011) Bisphenol A: an endocrine disruptor with widespread exposure and multiple effects. The Journal of steroid biochemistry and molecular biology, 127, $27-34$.

[272] Simerly, R. (2004) Anatomical substrates of hypothalamic integration. The rat nervous system, chap. 14, no. 1969.

[273] Schmittgen, T. D. and Livak, K. J. (2008) Analyzing real-time PCR data by the comparative CT method. Nature Protocols, 3, 1101-1108.

[274] Riant, E., Waget, A., Cogo, H., Arnal, J.-F., Burcelin, R., and Gourdy, P. (2009) Estrogens protect against high-fat diet-induced insulin resistance and glucose intolerance in mice. Endocrinology, 150, 2109-17.

[275] Schwartz, M. W., Seeley, R. J., Woods, S. C., Weigle, D. S., Campfield, L. a., Burn, P., and Baskin, D. G. (1997) Leptin increases hypothalamic proopiomelanocortin mRNA expression in the rostral arcuate nucleus. Diabetes, 46, 2119-23. 
[276] Mizuno, T. and Mobbs, C. (1999) Hypothalamic Agouti-Related Protein Messenger Ribonucleic Acid Is Inhibited by Leptin and Stimulated by Fasting. Endocrinology, 140, 814-817.

[277] Monje, L., Varayoud, J., Luque, E. H., and Ramos, J. G. (2007) Neonatal exposure to bisphenol A modifies the abundance of estrogen receptor alpha transcripts with alternative 5'-untranslated regions in the female rat preoptic area. The Journal of endocrinology, 194, 201-12.

[278] Khurana, S., Ranmal, S., and Ben-Jonathan, N. (2000) Exposure of Newborn Male and Female Rats to Environmental Estrogens: Delayed and Sustained Hyperprolactinemia and Alterations in Estrogen Receptor Expression. Endocrinology, 141, 4512-4517.

[279] Hirosawa, M., Minata, M., Harada, K. H., Hitomi, T., Krust, A., and Koizumi, A. (2008) Ablation of estrogen receptor alpha (ERalpha) prevents upregulation of POMC by leptin and insulin. Biochemical and biophysical research communications, $371,320-3$.

[280] Clegg, D. J., Brown, L. M., Woods, S. C., and Benoit, S. C. (2006) Gonadal hormones determine sensitivity to central leptin and insulin. Diabetes, 55, 978-87.

[281] Clegg, D. J., Riedy, C. a., Smith, K. a. B., Benoit, S. C., and Woods, S. C. (2003) Differential sensitivity to central leptin and insulin in male and female rats. Diabetes, 52, 682-7.

[282] Shi, H. and Clegg, D. J. (2009) Sex differences in the regulation of body weight. Physiology \& behavior, 97, 199-204.

[283] Harris, M., Aschkenasi, C., Elias, C. F., Chandrankunnel, a., Nillni, E. a., Bjø orbaek, C., Elmquist, J. K., Flier, J. S., and Hollenberg, a. N. (2001) Transcriptional regulation of the thyrotropin-releasing hormone gene by leptin and melanocortin signaling. The Journal of clinical investigation, 107, 111-20.

[284] Mullur, R., Liu, Y.-Y., and Brent, G. a. (2014) Thyroid hormone regulation of metabolism. Physiological reviews, 94, 355-82.

[285] Bouyer, K. and Simerly, R. B. (2013) Neonatal leptin exposure specifies innervation of presympathetic hypothalamic neurons and improves the metabolic status of leptin-deficient mice. The Journal of neuroscience, 33, 840-51.

[286] Bouret, S. G., Draper, S. J., and Simerly, R. B. (2004) Trophic action of leptin on hypothalamic neurons that regulate feeding. Science, 304, 108-10. 
[287] Wright, C. L., Schwarz, J. S., Dean, S. L., and McCarthy, M. M. (2010) Cellular mechanisms of estradiol-mediated sexual differentiation of the brain. Trends in endocrinology and metabolism: TEM, 21, 553-61.

[288] MacKay, H. and Abizaid, A. (2014) Embryonic development of the hypothalamic feeding circuitry: Transcriptional, nutritional, and hormonal influences. Molecular Metabolism, 3, 813-822.

[289] Cederroth, C. R., Vinciguerra, M., Kühne, F., Madani, R., Doerge, D. R., Visser, T. J., Foti, M., Rohner-Jeanrenaud, F., Vassalli, J.-D., and Nef, S. (2007) A phytoestrogen-rich diet increases energy expenditure and decreases adiposity in mice. Environmental health perspectives, 115, 1467-73.

[290] Ruhlen, R. L., Howdeshell, K. L., Mao, J., Taylor, J. a., Bronson, F. H., Newbold, R. R., Welshons, W. V., and vom Saal, F. S. (2008) Low phytoestrogen levels in feed increase fetal serum estradiol resulting in the "fetal estrogenization syndrome" and obesity in CD-1 mice. Environmental health perspectives, 116, 322-8.

[291] Andreoli, M. F., Stoker, C., Rossetti, M. F., Alzamendi, A., Castrogiovanni, D., Luque, E. H., and Ramos, J. G. (2014) Withdrawal of dietary phytoestrogens in adult male rats affects hypothalamic regulation of food intake, induces obesity and alters glucose metabolism. Molecular and cellular endocrinology.

[292] Mackay, H., Patterson, Z. R., Khazall, R., Patel, S., Tsirlin, D., and Abizaid, A. (2013) Organizational Effects of Perinatal Exposure to Bisphenol-A and Diethylstilbestrol on Arcuate Nucleus Circuitry Controlling Food Intake and Energy Expenditure in Male and Female CD-1 Mice. Endocrinology, 154, 1465-1475.

[293] Fernández, M., Bianchi, M., Lux-Lantos, V., and Libertun, C. (2009) Neonatal exposure to bisphenol a alters reproductive parameters and gonadotropin releasing hormone signaling in female rats. Environmental health perspectives, 117, 757-762.

[294] Monje, L., Varayoud, J., Muñoz-de Toro, M., Luque, E. H., and Ramos, J. G. (2010) Exposure of neonatal female rats to bisphenol A disrupts hypothalamic LHRH pre-mRNA processing and estrogen receptor alpha expression in nuclei controlling estrous cyclicity. Reproductive Toxicology, 30, 625-634.

[295] McLean, A. C., Valenzuela, N., Fai, S., and Bennett, S. a. L. (2012) Performing vaginal lavage, crystal violet staining, and vaginal cytological evaluation for mouse estrous cycle staging identification. Journal of visualized experiments, p. e4389. 
[296] Goldman, J., Murr, A., and Cooper, R. (2007) The Rodent Estrous Cycle: Characterization of Vaginal Cytology and Its Utility in Toxicological Studies. Birth Defects Research. Part B, 97, 84-97.

[297] Swart, I., Jahng, J. W., Overton, J. M., and Houpt, T. a. (2002) Hypothalamic NPY, AGRP, and POMC mRNA responses to leptin and refeeding in mice. American journal of physiology. Regulatory, integrative and comparative physiology, 283, R1020-R1026.

[298] Berdanier, C. (1981) Effects of Estrogen on the Responses of Male and Female Rats to Starvation-Refeeding. The Journal of nutrition, 30602, 1425-1429.

[299] Bouret, S. G., Bates, S. H., Chen, S., Myers, M. G., and Simerly, R. B. (2012) Distinct roles for specific leptin receptor signals in the development of hypothalamic feeding circuits. The Journal of neuroscience, 32, 1244-52.

[300] Proulx, K., Richard, D., and Walker, C. (2002) Leptin regulates appetite-related neuropeptides in the hypothalamus of developing rats without affecting food intake. Endocrinology, 143, 4683-4692.

[301] Bouret, S. G., Draper, S. J., and Simerly, R. B. (2004) Formation of projection pathways from the arcuate nucleus of the hypothalamus to hypothalamic regions implicated in the neural control of feeding behavior in mice. The Journal of neuroscience, 24, 2797-805.

[302] Jöhren, O., Neidert, S. J., Kummer, M., and Dominiak, P. (2002) Sexually dimorphic expression of prepro-orexin mRNA in the rat hypothalamus. Peptides, 23, 1177-80.

[303] Ramanathan, L. and Siegel, J. M. (2014) Gender differences between hypocretin/orexin knockout and wild type mice: age, body weight, body composition, metabolic markers, leptin and insulin resistance. Journal of neurochemistry, pp. 1-10.

[304] Leinninger, G. M., et al. (2011) Leptin action via neurotensin neurons controls orexin, the mesolimbic dopamine system and energy balance. Cell Metabolism, 14, 313-323.

[305] Opland, D., Sutton, A., Woodworth, H., Brown, J., Bugescu, R., Garcia, A., Christensen, L., Rhodes, C., Myers, M., and Leinninger, G. (2013) Loss of neurotensin receptor-1 disrupts the control of the mesolimbic dopamine system by leptin and promotes hedonic feeding and obesity. Molecular Metabolism, 2, 423434. 
[306] Goforth, P. B., Leinninger, G. M., Patterson, C. M., Satin, L. S., and Jr, M. G. M. (2014) Leptin Acts via Lateral Hypothalamic Area Neurotensin Neurons to Inhibit Orexin Neurons by Multiple GABA-Independent Mechanisms. Journal of neuroscience, 34, 11405-11415.

[307] Ishii, Y. and Bouret, S. G. (2012) Embryonic birthdate of hypothalamic leptinactivated neurons in mice. Endocrinology, 153, 3657-67.

[308] Chang, G.-Q., Gaysinskaya, V., Karatayev, O., and Leibowitz, S. F. (2008) Maternal high-fat diet and fetal programming: increased proliferation of hypothalamic peptide-producing neurons that increase risk for overeating and obesity. The Journal of Neuroscience, 28, 12107-19.

[309] Desai, M., Li, T., and Ross, M. G. (2011) Hypothalamic neurosphere progenitor cells in low birth-weight rat newborns: neurotrophic effects of leptin and insulin. Brain research, 1378, 29-42.

[310] Tiwari, S. K., Agarwal, S., and Seth, B. (2014) Inhibitory Effects of BisphenolA on Neural Stem Cells Proliferation and Differentiation in the Rat Brain Are Dependent on Wnt/ $\beta$-Catenin Pathway. Molecular neurobiology.

[311] Vogt, M., et al. (2014) Neonatal Insulin Action Impairs Hypothalamic Neurocircuit Formation in Response to Maternal High-Fat Feeding. Cell, pp. 495-509.

[312] Steculorum, S. M. and Bouret, S. G. (2011) Maternal diabetes compromises the organization of hypothalamic feeding circuits and impairs leptin sensitivity in offspring. Endocrinology, 152, 4171-9.

[313] Konieczna, J., García, A. P., Sánchez, J., Palou, M., Palou, A., and Picó, C. (2013) Oral leptin treatment in suckling rats ameliorates detrimental effects in hypothalamic structure and function caused by maternal caloric restriction during gestation. PloS one, $\mathbf{8}$, e81906.

[314] Picó, C., Oliver, P., Sánchez, J., Miralles, O., Caimari, A., Priego, T., and Palou, A. (2007) The intake of physiological doses of leptin during lactation in rats prevents obesity in later life. International journal of obesity, 31, 1199-209.

[315] Pennington, K. a., Harper, J. L., Sigafoos, A. N., Beffa, L. M., Carleton, S. M., Phillips, C. L., and Schulz, L. C. (2012) Effect of food restriction and leptin supplementation on fetal programming in mice. Endocrinology, 153, 4556-67.

[316] Vickers, M. H., Gluckman, P. D., Coveny, a. H., Hofman, P. L., Cutfield, W. S., Gertler, a., Breier, B. H., and Harris, M. (2005) Neonatal leptin treatment reverses developmental programming. Endocrinology, 146, 4211-6. 
[317] Ptak, A. and Gregoraszczuk, E. L. (2012) Bisphenol A induces leptin receptor expression, creating more binding sites for leptin, and activates the JAK/Stat, MAPK/ERK and PI3K/Akt signalling pathways in human ovarian cancer cell. Toxicology letters, 210, 332-7.

[318] Smith, J. T. and Waddell, B. J. (2003) Developmental changes in plasma leptin and hypothalamic leptin receptor expression in the rat: peripubertal changes and the emergence of sex differences. The Journal of endocrinology, 176, 313-319.

[319] Sawchenko, P. E. and Swanson, L. W. (1983) The Organization and Biochemical Specificity of Afferent Projections to the Paraventricular and Supraoptic Nuclei. Progress in Brain Research, 60, 19-29.

[320] Huo, L., Grill, H., and Bjø rbæk, C. (2006) Divergent regulation of proopiomelanocortin neurons by leptin in the nucleus of the solitary tract and in the arcuate hypothalamic nucleus. Diabetes, $\mathbf{5 5}$.

[321] Devaskar, S. U., Ollesch, C., Rajakumar, R. a., and Rajakumar, P. a. (1997) Developmental changes in ob gene expression and circulating leptin peptide concentrations. Biochemical and biophysical research communications, 238, 44-7.

[322] Bautista, C. J., Boeck, L., Larrea, F., Nathanielsz, P. W., and Zambrano, E. (2008) Effects of a maternal low protein isocaloric diet on milk leptin and progeny serum leptin concentration and appetitive behavior in the first 21 days of neonatal life in the rat. Pediatric Research, 63, 358-363.

[323] Han, J., Xu, J., Epstein, P. N., and Liu, Y. Q. (2005) Long-term effect of maternal obesity on pancreatic beta cells of offspring: Reduced beta cell adaptation to high glucose and high-fat diet challenges in adult female mouse offspring. Diabetologia, 48, 1810-1818.

[324] Bayol, S. a., Simbi, B. H., Bertrand, J. a., and Stickland, N. C. (2008) Offspring from mothers fed a 'junk food' diet in pregnancy and lactation exhibit exacerbated adiposity that is more pronounced in females. The Journal of physiology, 586, 3219-3230.

[325] Dearden, L. and Balthasar, N. (2014) Sexual dimorphism in offspring glucosesensitive hypothalamic gene expression and physiological responses to maternal high-fat diet feeding. Endocrinology, 155, 2144-2154.

[326] Vickers, M. H., Gluckman, P. D., Coveny, A. H., Hofman, P. L., Cutfield, W. S., Gertler, A., Breier, B. H., and Harris, M. (2008) The effect of neonatal leptin treatment on postnatal weight gain in male rats is dependent on maternal nutritional status during pregnancy. Endocrinology, 149, 1906-13. 
[327] Xu, X. H., Zhang, J., Wang, Y. M., Ye, Y. P., and Luo, Q. Q. (2010) Perinatal exposure to bisphenol-A impairs learning-memory by concomitant downregulation of $\mathrm{N}$-methyl-d-aspartate receptors of hippocampus in male offspring mice. Hormones and Behavior, 58, 326-333.

[328] Walker, C. and Long, H. (2007) Long-lasting effects of elevated neonatal leptin on rat hippocampal function, synaptic proteins and NMDA receptor subunits. Journal of neuroscience research, 828, 816-828.

[329] Ahima, R. S., Dushay, J., Flier, S. N., Prabakaran, D., and Flier, J. S. (1997) Leptin accelerates the onset of puberty in normal female mice. Journal of Clinical Investigation, 99, 391-395.

[330] Cheung, C. C., Thornton, J. E., Kuijper, J. L., Weigle, D. S., Clifton, D. K., and Steiner, R. A. (1997) Leptin is a metabolic gate for the onset of puberty in the female rat. Endocrinology, 138, 855-858.

[331] Franssen, D., Ioannou, Y. S., Alvarez-real, A., Gerard, A., Mueller, J. K., Heger, S., Bourguignon, J.-P., and Parent, A.-S. (2014) Pubertal timing after neonatal diethylstilbestrol exposure in female rats: neuroendocrine vs peripheral effects and additive role of prenatal food restriction. Reproductive toxicology, 44, 63-72.

[332] Shi, H., Strader, A. D., Sorrell, J. E., Chambers, J. B., Woods, S. C., and Seeley, R. J. (2008) Sexually different actions of leptin in proopiomelanocortin neurons to regulate glucose homeostasis. American journal of physiology. Endocrinology and metabolism, 294, E630-9.

[333] Sánchez, J., Oliver, P., Miralles, O., Ceresi, E., Picó, C., and Palou, A. (2005) Leptin orally supplied to neonate rats is directly uptaken by the immature stomach and may regulate short-term feeding. Endocrinology, 146, 2575-82.

[334] Herrera, E., Lasunción, M. a., Huerta, L., and Martín-Hidalgo, a. (2000) Plasma leptin levels in rat mother and offspring during pregnancy and lactation. Biology of the neonate, 78, 315-320.

[335] Kawai, M., Yamaguchi, M., Murakami, T., Shima, K., Murata, Y., and Kishi, K. (1997) The placenta is not the main source of leptin production in pregnant rat: gestational profile of leptin in plasma and adipose tissues. Biochemical and biophysical research communications, 240, 798-802.

[336] Nicholas, K. R. and Hartmann, P. E. (1991) Milk secretion in the rat: progressive changes in milk composition during lactation and weaning and the effect of diet. Comparative biochemistry and physiology. A, Comparative physiology, 98, 535-542. 
[337] Luckey, T. D., Mende, T. J., and Pleasants, J. (1954) The physical and chemical characterization of rat's milk. The Journal of nutrition, 54, 345-359.

[338] Del Prado, M., Delgado, G., and Villalpando, S. (1997) Maternal lipid intake during pregnancy and lactation alters milk composition and production and litter growth in rats. The Journal of nutrition, 127, 458-462.

[339] Rolls, B. a., Gurr, M. I., van Duijvenvoorde, P. M., Rolls, B. J., and Rowe, E. a. (1986) Lactation in lean and obese rats: effect of cafeteria feeding and of dietary obesity on milk composition. Physiology \& behavior, 38, 185-190.

[340] Grigor, M. R., Allan, J. E., Carrington, J. M., Carne, a., Geursen, a., Young, D., Thompson, M. P., Haynes, E. B., and Coleman, R. a. (1987) Effect of dietary protein and food restriction on milk production and composition, maternal tissues and enzymes in lactating rats. The Journal of nutrition, 117, 1247-1258.

[341] Rasmussen, K. M. (1998) Effects of under- and overnutrition on lactation in laboratory rats. The Journal of nutrition, 128, 390S-393S.

[342] Lenz, K. M. and McCarthy, M. M. (2010) Organized for sex - steroid hormones and the developing hypothalamus. The European journal of neuroscience, 32, 2096104.

[343] Nohara, K., Laque, A., Allard, C., Münzberg, H., and Mauvais-Jarvis, F. (2014) Central mechanisms of adiposity in adult female mice with androgen excess. Obesity, 22, 1477-84.

[344] Freeman, E., Bloom, D., and McGuire, E. (2001) A brief history of testosterone. The Journal of Urology, 49, 51-55.

[345] Nohynek, G. J., Borgert, C. J., Dietrich, D., and Rozman, K. K. (2013) Endocrine disruption: Fact or urban legend? Toxicology Letters, 223, 295-305.

[346] Haubruge, E., Petit, F., and Gage, M. J. (2000) Reduced sperm counts in guppies (Poecilia reticulata) following exposure to low levels of tributyltin and bisphenol A. Proceedings. Biological sciences / The Royal Society, 267, 2333-7.

[347] Sohoni, P., et al. (2001) Reproductive Effects of Long-Term Exposure to Bisphenol A in the Fathead Minnow ( Pimephales promelas ). Environmental Science \& Technology, 35, 2917-2925.

[348] Levy, G. (2004) Bisphenol A induces feminization in Xenopus laevis tadpoles. Environmental Research, 94, 102-111. 
[349] Klimentidis, Y. C., et al. (2011) Canaries in the coal mine: a cross-species analysis of the plurality of obesity epidemics. Proceedings. Biological sciences / The Royal Society, $278,1626-32$.

[350] Wang, T., et al. (2012) Urinary bisphenol A (BPA) concentration associates with obesity and insulin resistance. The Journal of clinical endocrinology and metabolism, 97, E223-7.

[351] Robledo, C., Peck, J. D., Stoner, J. a., Carabin, H., Cowan, L., Koch, H. M., and Goodman, J. R. (2013) Is bisphenol-A exposure during pregnancy associated with blood glucose levels or diagnosis of gestational diabetes? Journal of toxicology and environmental health. Part A, 76, 865-73.

[352] Oppeneer, S. J. and Robien, K. (2014) Bisphenol A exposure and associations with obesity among adults: a critical review. Public health nutrition, pp. 1-17.

[353] Padmanabhan, V., Siefert, K., Ransom, S., Johnson, T., Pinkerton, J., Anderson, L., Tao, L., and Kannan, K. (2008) Maternal bisphenol-A levels at delivery: a looming problem? Journal of perinatology, 28, 258-63.

[354] McCarthy, M. M., Pickett, L. a., VanRyzin, J. W., and Kight, K. E. (2015) Surprising Origins of Sex Differences in the Brain. Hormones and Behavior.

[355] Bonthuis, P. J., Cox, K. H., Searcy, B. T., Kumar, P., Tobet, S., and Rissman, E. F. (2010) Of mice and rats: Key species variations in the sexual differentiation of brain and behavior. Frontiers in Neuroendocrinology, 31, 341-358.

[356] McCarthy, M. (2010) Sex and the Developing Brain, vol. 1.

[357] Lieberburg, I., Krey, L. C., and McEwen, B. S. (1979) Sex differences in serum testosterone and in exchangeable brain cell nuclear estradiol during the neonatal period in rats. Brain Research, 178, 207-212.

[358] Vaag, a. a., Grunnet, L. G., Arora, G. P., and Brø ns, C. (2012) The thrifty phenotype hypothesis revisited. Diabetologia, 55, 2085-8.

[359] Forsdahl, A. (1977) Are poor living conditions in childhood and adolescence an important risk factor for arteriosclerotic heart disease? British Journal of Preventative and Social Medicine, pp. 91-95.

[360] Hales, C. and Barker, D. (1992) Type 2 (non-insulin-dependent) diabetes mellitus: the thrifty phenotype hypothesis. Diabetologia, 2. 
[361] Barker, D., Hales, C., Fall, C., and Osmond, C. (1993) Type 2 (non-insulindependent) diabetes mellitus, hypertension and hyperlipidaemia (syndrome $\mathrm{X}$ ): relation to reduced fetal growth. Diabetologia, 2, 62-67.

[362] Clausen, T., Mathiesen, E., Hansen, T., Pedersen, O., Jensen, D., Lauenborg, J., and Damm, P. (2008) High prevalence of type 2 diabetes and pre-diabetes in adult offspring of women with gestational diabetes mellitus or type 1 diabetes the role of intrauterine. Diabetes Care, 31 .

[363] Freinkel, N. (1980) Gestational Diabetes 1979: Philosophical and Practical Aspects of a Major Public Health Problem. Diabetes care, 3, 1979-1981.

[364] Szczepankiewicz, D., Wojciechowicz, T., Kaczmarek, P., and Nowak, K. W. (2006) Leptin and its receptors in the course of pregnancy in the rat. International journal of molecular medicine, 17, 95-9.

[365] Smith, J. T. (2003) Leptin Distribution and Metabolism in the Pregnant Rat: Transplacental Leptin Passage Increases in Late Gestation but Is Reduced by Excess Glucocorticoids. Endocrinology, 144, 3024-3030.

[366] Schubring, C., Siebler, T., Kratzsch, J., Englaro, P., Blum, W. F., Triep, K., and Kiess, W. (1999) Leptin serum concentrations in healthy neonates within the first week of life: relation to insulin and growth hormone levels, skinfold thickness, body mass index and weight. Clinical Endocrinology, 51, 199-204.

[367] Molvarec, A., Szarka, A., Walentin, S., Beko, G., Karádi, I., Prohászka, Z., and Rigó, J. (2011) Serum leptin levels in relation to circulating cytokines, chemokines, adhesion molecules and angiogenic factors in normal pregnancy and preeclampsia. Reproductive biology and endocrinology : RB\&E, 9, 124.

[368] Castellano Filho, D. S., do Amaral Correa, J. O., Dos Santos Ramos, P., de Oliveira Montessi, M., Aarestrup, B. J. a. V., and Aarestrup, F. M. (2013) Body weight gain and serum leptin levels of non-overweight and overweight/obese pregnant women. Medical science monitor, 19, 1043-9.

[369] Lepercq, J. and Challier, J. (2001) Prenatal Leptin Production: Evidence That Fetal Adipose Tissue Produces Leptin. Journal of Clinical Endocrinology \& Metabolism, 86, 2409-2413.

[370] Linnemann, K., Malek, a., Sager, R., Blum, W. F., Schneider, H., and Fusch, C. (2000) Leptin production and release in the dually in vitro perfused human placenta. The Journal of clinical endocrinology and metabolism, 85, 4298-301. 
[371] Atanassova, P. and Popova, L. (2000) Leptin Expression during the Differentiation of Subcutaneous Adipose Cells of Human Embryos in situ. Cells Tissues Organs.

[372] Matsuda, J., Yokota, I., Iida, M., Murakami, T., Yamada, M., Saijo, T., Naito, E., Ito, M., Shima, K., and Kuroda, Y. (1999) Dynamic changes in serum leptin concentrations during the fetal and neonatal periods. Pediatric research, 45, 71-75.

[373] Cetin, I., Morpurgo, P. S., Radaelli, T., Taricco, E., Cortelazzi, D., Bellotti, M., Pardi, G., and Beck-Peccoz, P. (2000) Fetal plasma leptin concentrations: relationship with different intrauterine growth patterns from 19 weeks to term. Pediatric research, 48, 646-51.

[374] Jaquet, D., Leger, J., and Levy-Marchal, C. (1998) Ontogeny of Leptin in Human Fetuses and Newborns : Effect of Intrauterine Growth Retardation on Serum Leptin Concentrations. Journal of Clinical Endocrinology \& Metabolism, 83, 1243 1246.

[375] Yildiz, L., Avci, B., and Ingeç, M. (2002) Umbilical cord and maternal blood leptin concentrations in intrauterine growth retardation. Clinical chemistry and laboratory medicine, $40,1114-7$.

[376] Lea, R. G., Howe, D., Hannah, L. T., Bonneau, O., Hunter, L., and Hoggard, N. (2000) Placental leptin in normal, diabetic and fetal growth-retarded pregnancies. Molecular buman reproduction, 6, 763-9.

[377] Ong, K., Ahmed, M., and Sherriff, A. (1999) Cord blood leptin is associated with size at birth and predicts infancy weight gain in humans. Journal of Clinical Endocrinology \& Metabolism, 84.

[378] Miralles, O., Sánchez, J., Palou, A., and Picó, C. (2006) A physiological role of breast milk leptin in body weight control in developing infants. Obesity.

[379] Savino, F., Liguori, S. a., Petrucci, E., Lupica, M. M., Fissore, M. F., Oggero, R., and Silvestro, L. (2010) Evaluation of leptin in breast milk, lactating mothers and their infants. European journal of clinical nutrition, 64, 972-7.

[380] Doneray, H., Orbak, Z., and Yildiz, L. (2009) The relationship between breast milk leptin and neonatal weight gain. Acta paediatrica, 98, 643-7.

[381] Briffa, J. F., McAinch, A. J., Romano, T., Wlodek, M. E., and Hryciw, D. H. (2014) Leptin in pregnancy and development: a contributor to adulthood disease? American Journal of Physiology, Endocrinology, and Metabolism. 
C2015 - Harry MacKay

Typeset in $\mathrm{LT}_{\mathrm{E}} \mathrm{X}$ 